\title{
Characterisation of Detached Plasmas on the MAST Tokamak
}

\author{
James Robert HARRISON
}

Supervisors

Dr. K. J. Gibson

Dr. A. KIRK

(Dr. S. W. LisGo)

A thesis submitted to the University of York in accordance with the requirements for the Degree of Doctor of Philosophy.

\author{
Plasma Physics \& Fusion Group \\ Department of Physics \\ University of York \\ Heslington, York Y010 5DD
}

September 2010 



\section{Abstract}

The mitigation of the steady-state heat loading to plasma-facing components in the divertor structures is an essential prerequisite for the operation of next-generation conventional and tight aspect-ratio Tokamaks. Currently, the most attractive means of reducing this heat loading is the so-called "detached" regime, which is the primary focus of this study.

This thesis describes work that was carried out on the MAST tokamak to accurately characterise the plasma conditions within divertor plasmas, and to test the validity of those measurements. To assist in this study, an interpretive code, OSM [1], has been upgraded with additional numerical schemes to ascertain the dominant mechanisms governing parallel scrape-off layer transport including flux expansion, dynamic viscosity and cross-field drift motion in attached conditions. It has been found that the invocation of flux expansion, parallel viscosity and diamagnetic drift motion assist the OSM numerical scheme to converge in the presence of (static+dynamic) parallel pressure gradients often observed between the outboard midplane and lower outer divertor leg. It has also been found that the ion pressure, which is currently unknown, could resolve the observed pressure discrepancy.

Line-of-sight spectroscopy of high-n Balmer emission lines has been used to ascertain line averaged $T_{e}$ and line integrated $n_{e}$ during the detached phase of MAST discharges with high spatial resolution $(\approx 7 \mathrm{~mm})$ to determine electron static pressure profiles along flux contours. Furthermore, narrow-bandwidth imaging spectroscopy of $\mathrm{D}_{\alpha}, \mathrm{D}_{\gamma}, \mathrm{CII}$ and CIII emission during the onset and sustainment of detachment has been carried out with high temporal $(5 \mathrm{kHz})$ and spatial $(\approx 3 \mathrm{~mm})$ resolution. These data have been combined with ion flux measurements to the divertor target plates using embedded Langmuir probes (6-9mm radial resolution) and upstream Thomson scattering measurements of $n_{e}, T_{e}$. The data was input into the OSM code, coupled with the kinetic neutral transport code EIRENE [2], to check the experimental data set for internal consistency. The experimental data collected has been used to reconstruct the plasma conditions within the detached divertor leg, heavily constrained by experimental data. EIRENE calculations with re-constructed plasma conditions are able to reproduce experimentally observed $\mathrm{D}_{\gamma} / \mathrm{D}_{\alpha}$ line emission ratios to within a factor 
of 2 within the recombining region. 


\section{Acknowledgments}

The work presented in this thesis is the culmination of the work of many people, who I gratefully acknowledge here. Foremost, I thank Dr. Steven Lisgo for his guidance, enthusiasm and assistance over the last 4 years. It is only on reflection that I appreciate how he kept this work on track, whilst encouraging me to explore my own ideas and how doing so has enhanced the quality of the work presented here. Drs. Andrew Kirk and Geoff Fishpool also deserve special thanks for their guidance, and above all, their understanding in the final months of this work. I also owe great thanks to Dr. Kieran Gibson for steering this project from its inception, and to whom I owe the opportunity to pursue research in this field.

I am also thankful to the many people from CCFE who have contributed to this work over the years, especially John Dowling, for his help over the years and enduring my endless questions and bright ideas, I hope he has a long and happy retirement. I am also eternally grateful to Drs Neil Conway, Clive Michael, Stuart Robertson and Hendrik Meyer for their assistance with the multichord spectrometer and to Dr Maarten De Bock for kindly loaning me the AM-510 spectrometer. Many thanks also to Dr Patrick Tamain, for helping me with some of the scarier aspects of edge transport theory. Andrew Thornton also deserves a special mention for his patience, humour and seemingly boundless supply of common sense. Thanks also to the MAST session leaders, Drs Geoff Cunningham, Richard Martin, Graham Naylor, Sergei Shibaev, Eric Nardon, John Storrs and Dave Taylor for leading my normally chaotic detachment experiments. I am also indebted to Drs Rory Scannell, Alex Carter, Minh-Duc Hua, Seb Tallents, Adam Foster and Sarah Newton for their advice and assistance. Thanks also to Dr. Scott Allan, whose patience and good humour have made the creation of this thesis much more enjoyable than it would have otherwise been.

My deepest thanks go to my parents, who have supported and encouraged me through this work. Equal thanks go to Olivia, whose support, especially during the writing of this thesis, has been invaluable. 


\section{Declaration}

I declare that the work in this thesis was carried out in accordance with the Regulations of the University of York. The work is original, except where indicated by special reference in the text, and no part of the thesis has been submitted for any other academic award. Any views expressed in the thesis are those of the author.

Signed:

Date: 


\section{Contents}

1 Introduction 1

1.1 Introduction to Fusion . . . . . . . . . . . . . . . . . 1

1.2 Introduction to Tokamaks . . . . . . . . . . . . . . . . 2

1.2.1 The Spherical Tokamak . . . . . . . . . . . . . . . . . 4

1.3 Plasma-Wall Interactions $\ldots \ldots \ldots \ldots$

1.3.1 Limiter and Divertor Configurations . . . . . . . . . . . 5

1.3.2 Divertor Detachment . . . . . . . . . . . . . 7

1.4 Thesis Summary . . . . . . . . . . . . . . . . . . 8

2 Divertor Physics $\quad 10$

2.1 Divertor Geometry . . . . . . . . . . . . . . . . . . 10

2.2 Sheath Physics and the Plasma/Surface Boundary . . . . . . . . . . 12

2.3 The Two-Point Model . . . . . . . . . . . . . . . . . . 16

2.4 Divertor Operating Regimes . . . . . . . . . . . . . . . . . 18

2.4.1 The Sheath-Limited Regime . . . . . . . . . . . . . 18

2.4.2 The Conduction Limited Regime . . . . . . . . . . . . . . . . . . . . . . . . . . .

2.4 .3 Detachment . . . . . . . . . . . . . . . . . . 20

2.5 Properties of Detached Plasmas . . . . . . . . . . . . . . . . 21

2.5.1 Stability of Detachment . . . . . . . . . . . . . . 23

2.6 Edge Physics Modelling . . . . . . . . . . . . . . . . . . . . 24

2.7 The Onion-Skin Model . . . . . . . . . . . . . . . . . . 25

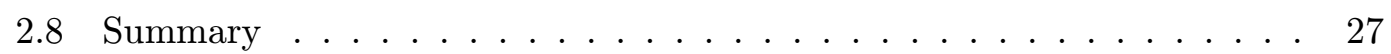

3 The Mega Ampère Spherical Tokamak 28

3.1 Introduction to MAST . . . . . . . . . . . . . . . . . 28

3.2 Edge \& Divertor Physics on MAST . . . . . . . . . . . . . . . . . 29

3.2 .1 The MAST Divertor . . . . . . . . . . . . . . . 29

$3.2 .2 \quad$ Plasma Equilibria . . . . . . . . . . . . . . . . . . . . 29

3.3 Edge Diagnostics . . . . . . . . . . . . . . . . . . . . . . . . . .

3.3 .1 Langmuir Probes . . . . . . . . . . . . . . . . . . 30 
$3.3 .2 \quad$ Spectroscopy . . . . . . . . . . . . . . . . . . . 33

3.3.3 Spectroscopic $\mathrm{T}_{\mathrm{e}}$ Determination $\ldots \ldots \ldots . \ldots 36$

3.3.4 Spectroscopic Line Integrated $\mathrm{n}_{\mathrm{e}}$ Determination . . . . . . . . . . 39

3.3.5 Thomson Scattering . . . . . . . . . . . . . . . . 43

$3.3 .6 \quad$ Infrared Cameras . . . . . . . . . . . . . . . . . . . . 44

3.3 .7 Visible Imaging . . . . . . . . . . . . . . . . . . . . 46

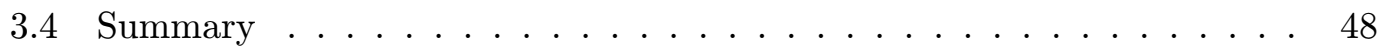

4 OSM Physics Additions $\quad 50$

4.1 The Original Model . . . . . . . . . . . . . . . . . . . . . . . . . . . . . . 50

4.2 SOL Potentials \& Currents . . . . . . . . . . . . . . . . 51

4.2 .1 SOL Potentials . . . . . . . . . . . . . . . 51

4.2 .2 Thermoelectric Currents . . . . . . . . . . . . . . . . 52

4.2 .3 Pfirsch-Schlüter Currents . . . . . . . . . . . . . . . 55

4.3 Flux Expansion Terms . . . . . . . . . . . . . . . . . . . . . 57

4.4 Parallel Viscosity . . . . . . . . . . . . . . . . . . . 64

4.5 Cross-Field Drifts . . . . . . . . . . . . . . . . . . 68

4.5 .1 The $\mathrm{E} \times \mathrm{B}$ Drift $\ldots \ldots \ldots \ldots \ldots \ldots$

4.5.2 The Diamagnetic Drift . . . . . . . . . . . . . 75

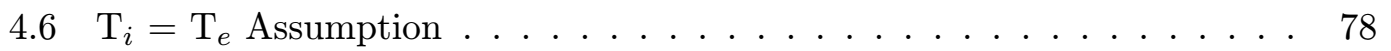

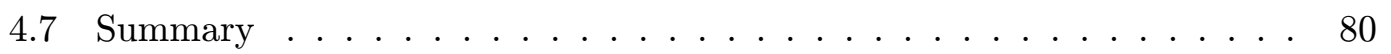

5 Detachment on MAST $\quad \mathbf{8 2}$

5.1 Introduction . . . . . . . . . . . . . . . . . . . . 82

5.2 Two-Point Modelling . . . . . . . . . . . . . . . . . 83

5.3 Experimental Observations . . . . . . . . . . . . . . 87

5.3 .1 Operational Limits . . . . . . . . . . . . . . . . . . . . . 87

5.3 .2 Thomson Scattering . . . . . . . . . . . . . . . 89

5.3 .3 Target Langmuir Probes . . . . . . . . . . . . . . . . . . . 92

5.3.4 InfraRed Thermography . . . . . . . . . . . . . . . . . 94

5.3.5 Divertor Line-of-Sight Spectroscopy . . . . . . . . . . . . . 96

5.3 .6 Imaging Spectroscopy _ . . . . . . . . . . . . . . . . . . . . 99

5.4 Interpretive Detachment Modelling . . . . . . . . . . . . . . . . . 107

$5.4 .1 \quad$ OSM Setup . . . . . . . . . . . . . . . 107

$5.4 .2 \quad$ Results . . . . . . . . . . . . . . . . . . 109

5.5 Summary . . . . . . . . . . . . . . . . . . . 112

6 Conclusions and Future Work 118

6.0 .1 Conclusions . . . . . . . . . . . . . . . . . . 118 
6.0 .2 Future Work . . . . . . . . . . . . . . . . . . . 120

A OSM Solver Development $\quad 122$

A.1 The Runge-Kutta Method . . . . . . . . . . . . . . . . . . . . 122

A.2 The Finite-Volume Method . . . . . . . . . . . . . . . . 123

A.2.1 The Hybrid Scheme . . . . . . . . . . . . . . . . 125

A.2.2 Time-Dependent Equations . . . . . . . . . . . . . . 130

A.2.3 The MUSCL Scheme . . . . . . . . . . . . . . . . 131

A.3 The Time-Dependent Original Model . . . . . . . . . . . . . . . . . . 134

A.4 The Extended Time-Dependent Model . . . . . . . . . . . . . . . 138

References

139 


\section{List of Figures}

1.1 Fusion cross-sections as a function of Deuteron energy . . . . . . . . 2

1.2 Schematic of a generic tokamak . . . . . . . . . . . . 3

1.3 Schematic of a Tokamak limiter . . . . . . . . . . . . . . . 6

1.4 Tokamak divertor schematic . . . . . . . . . . . . . 7

2.1 Open and closed divertor schematics . . . . . . . . . . . . . . . . 11

2.2 Horizontal and vertical target ionisation distributions . . . . . . . . . 11

2.3 Geometry used in derivation of the Bohm criterion . . . . . . . . . . . 13

2.4 Geometry of the two-point model . . . . . . . . . . . . . . 16

2.5 Distribution of particle sources in the sheath-limited and conductionlimited regimes . . . . . . . . . . . . . . . . . 20

2.6 JET detachment evolution data . . . . . . . . . . . . . . . . . 22

2.7 Simplified schematic of divertor detachment . . . . . . . . . . . . . . 22

2.8 JET bolometric data of detachment . . . . . . . . . . . . . . . . 24

2.9 Results of an OSM simulation of MAST shot 13018 . . . . . . . . . 26

3.1 Engineering schematic of MAST _ . . . . . . . . . . . . . 29

3.2 MAST magnetic equilibria . . . . . . . . . . . . . . . . . . . . . . . . . 30

3.3 Description of Tokamak geometry . . . . . . . . . . . . . . . . 31

3.4 Schematic Langmuir probe I-V characteristic . . . . . . . . . . . . . 32

3.5 Input optics for SP2300i spectrometer . . . . . . . . . . . . . . . . 34

3.6 Measured high-n Balmer emission lines in MAST . . . . . . . . . . 35

3.7 PLTE calculations . . . . . . . . . . . . . . . . . . . . 40

3.8 Simulated Stark broadened spectrum . . . . . . . . . . . . . . 41

3.9 Stark fitting of data from $300 \mathrm{~mm}$ and $1000 \mathrm{~mm}$ spectrographs . . . . . . 42

3.10 Laser path and optics of the MAST Thomson scattering diagnostic . . . 44

3.11 Example data from the upgraded MAST Thomson scattering diagnostic 45

3.12 Comparison of simulated Bremsstrahlung and blackbody emissivities . . 46

3.13 CAD model of DivCam . . . . . . . . . . . . . . . . . . 47

3.14 A disruption on MAST as seen by DivCam . . . . . . . . . . . . 47 
4.1 Illustration of thermoelectric current generation . . . . . . . . . . . 53

4.2 Measured and calculated thermoelectric currents on MAST . . . . . . 55

4.3 Calculated Pfirsch-Schlüter currents _ . . . . . . . . . . . . 57

4.4 EFIT data of MAST magnetic field components . . . . . . . . . . 58

4.5 OSM calculated magnetic field strength and $\nabla_{\|} B / B \ldots \ldots$. . . . . 59

4.6 Magnetic grid used to simulate shot 24861 at $240 \mathrm{~ms} \ldots \ldots$. . . . . . 60

4.7 Comparison of different implementations of flux expansion terms . . . . 61

4.8 OSM-EIRENE calculations of the effects of magnetic flux expansion . . 62

4.9 Distribution of sources and sinks in flux expansion analysis . . . . . . 63

4.10 The distribution of momentum sources in MAST shot 24861. The momentum source due to the flux expansion terms is given by solver_exp

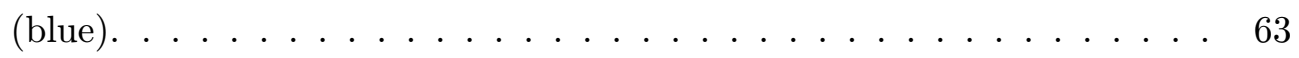

4.11 Effects of flux expansion on plasma potential profiles . . . . . . . . . 64

4.12 OSM-EIRENE calculations of the effects of parallel viscosity . . . . 66

4.13 Calculation of ion parallel stress tensor component . . . . . . . . 67

4.14 Distribution of sources and sinks in parallel viscosity analysis . . . . . . 67

4.15 Co-ordinate system used in OSM drift analysis . . . . . . . . . . . 69

4.16 Comparison of analytical estimates and OSM calculations of $\vec{E} \times \vec{B} \mathrm{drift}$ velocities. . . . . . . . . . . . . . . . . . 72

4.17 Radial $\vec{E} \times \vec{B}$ drift velocity sensitivity analysis . . . . . . . . . . 72

4.18 Effects of $\vec{E} \times \vec{B}$ drift motion on density and flow profiles . . . . . . 73

4.19 Effects of $\vec{E} \times \vec{B}$ drift motion on total pressure profiles . . . . . . . . 74

4.20 Distrubution of sources and inks in $\vec{E} \times \vec{B}$ drift analysis . . . . . . . . 74

4.21 Effects of the diamagnetic drift on parallel density profiles . . . . . . . 77

4.22 The effects of the diamagnetic drift on parallel Mach number and pres-

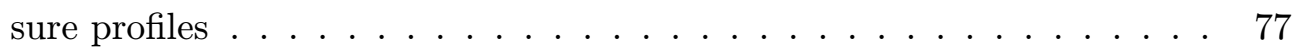

4.23 The effects of the Pfirsch-Schlüter current on parallel potential profiles . 78

4.24 Distribution of particle sources and sinks in diamagnetic drift analysis . 79

4.25 OSM-EIRENE simulations of the effects of varying $\mathrm{T}_{i} / \mathrm{T}_{e}(\gamma) \ldots 80$

5.1 The original and improved MAST divertors . . . . . . . . . . . . 83

5.2 Calculation of momentum loss rates in the two-point model . . . . . . . 84

5.3 Two-point model simulation of a density ramp experiment . . . . . . . 87

5.4 Two-point model power balance during a density ramp experiment . . . 88

5.5 ADAS calculation of $\mathrm{D}_{\gamma} / \mathrm{D}_{\alpha}$ eimissivity line ratio $\ldots \ldots \ldots$. . . . 89

5.6 Hugill diagram of shot $25028 \ldots \ldots \ldots \ldots$

5.7 Summary of shots 24861,24867 and $25028 \ldots \ldots \ldots$

5.8 Layout of principal detachment diagnostics . . . . . . . . . . . . . . . . . 91

5.9 Upstream Thomson scattering data input into OSM . . . . . . . . . . 93 
5.10 Temporal variation in smoothed ion saturation current data at the divertor targets . . . . . . . . . . . . . . . . . . 94

5.11 Time-averaged Langmuir probe data input into OSM . . . . . . . . . . . 95

5.12 IR camera data indicating emission from the bulk plasma . . . . . . . 95

5.13 Tangency locations of line-of-sight spectroscopic viewing chords . . . . . 96

5.14 Raw line-of-sight spectroscoy data for $\mathrm{T}_{e}$ determination $\ldots . . . .98$

5.15 Line integrated $\mathrm{n}_{e}$ and line averaged $\mathrm{T}_{e}$ profile from line-of-sight spectroscopy . . . . . . . . . . . . . . . . . . . . 99

5.16 Fractional abundance of carbon charge states . . . . . . . . . . . 100

5.17 Evolution of CII and CIII emission during detachment . . . . . . . . . 101

5.18 Tomographically inverted $D_{\alpha}$ emission from shot 25028 at $312 \mathrm{~ms}$. . . . 102

5.19 Tomographically inverted $\mathrm{D}_{\gamma}$ emission from shot 25028 at $312 \mathrm{~ms}$. . . . 103

5.20 Tomographically inverted CII $(514 \mathrm{~nm})$ emission from shot 25028 at $312 \mathrm{~ms} 104$

5.21 Tomographically inverted CIII (465nm) emission from shot 25028 at $312 \mathrm{~ms} 105$

$5.22 \mathrm{D}_{\gamma} / \mathrm{D}_{\alpha}$ line intensities as measured by DivCam in shots 25028 and 24867106

5.23 Spectra of possible contamination of spatially resolved $\mathrm{D}_{\gamma}$ emission measurements . . . . . . . . . . . . . . . . 106

5.24 Approximate $\mathrm{T}_{e}$ contours using tomographically inverted CII and CIII emission . . . . . . . . . . . . . . . . . . . . . 108

5.25 Experimental data input into OSM to simulate shot 25028 at $312 \mathrm{~ms}$. . 110

5.26 OSM-EIRENE plasma solution for shot 25018 at $312 \mathrm{~ms}$ along the separatrix $\operatorname{ring} \ldots \ldots \ldots \ldots \ldots$

$5.272 \mathrm{D} n_{e}, T_{e}$ contours of the OSM-EIRENE plasma solution of shot 25028

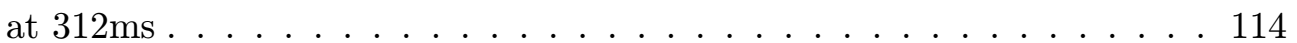

5.28 EIRENE calculations of $\mathrm{D}_{\alpha}$ and $\mathrm{D}_{\gamma}$ emissivities from shot 25028 at $312 \mathrm{~ms} 115$

5.29 Comparison of tomographically inverted $\mathrm{D}_{\gamma} / \mathrm{D}_{\alpha}$ emission measured by DivCam and an OSM-EIRENE simulation. . . . . . . . . . . 116

5.30 Contributions to calculated $\mathrm{D}_{\alpha}$ and $\mathrm{D}_{\gamma}$ emission along the separatrix ring calculated by EIRENE . . . . . . . . . . . . . . . 116

5.31 Contributions to calculated $\mathrm{D}_{\alpha}$ and $\mathrm{D}_{\gamma}$ emission along a flux tube intersecting the lower inboard $\mathrm{D}_{2}$ gas puff calculated by EIRENE $\ldots \ldots$. . 116

A.1 Euler method description . . . . . . . . . . . . . . . . . . 123

A.2 Original OSM and Runge-Kutta solver comparison . . . . . . . . . . . 124

A.3 Geometry used in the finite-volume discetisation. Uppercase letters indicate computational cells, lowercase letters indicate cell faces. . . . . 124

A.4 Instabilities in the 2nd order central differencing scheme . . . . . . . 127

A.5 The 1st order upwind scheme . . . . . . . . . . . . . . 128

A.6 The hybrid scheme . . . . . . . . . . . . . . . . . . . . . . 129 
A.7 Electrostatic potential and field profiles using a hybrid scheme solver . . 129

A.8 Schematic of the CFL stability condition . . . . . . . . . . . . 131

A.9 Sweby diagram of flux limiters used in this study . . . . . . . . . . . 132

A.10 Performance of the TVD-MUSCL scheme . . . . . . . . . . . 133

A.11 TVD-MUSCL solver benchmarking . . . . . . . . . . . . . . . . 135

A.12 Comparison of original OSM and TVD-MUSCL solutions to the original transport equations . . . . . . . . . . . . . . 136

A.13 Density and ion velocity evolution during a TVD-MUSCL solver run . . 137

A.14 Effects of choice of flux limiter on plasma solutions . . . . . . . . . . . 137 


\section{List of Tables}

1.1 Engineering parameters of START and MAST Tokamaks. . . . . . . . 5

5.1 Description of the principal discharges in this study . . . . . . . . . . 91

5.2 Neutral collision processes modelled by EIRENE . . . . . . . . . . . 112 


\section{Chapter 1}

\section{Introduction}

In this chapter, a brief introduction to fusion as a potential future energy source is given. This is followed by an overview of the Tokamak concept, with particular attention focused on the spherical Tokamak, MAST. An introduction to plasma-surface interactions and their role in magnetic confinement fusion research is given. Finally, an overview of the organisation of this thesis is given.

\subsection{Introduction to Fusion}

For the majority of the 20th century, the term electricity generation was almost synonymous with the burning of fossil fuels. However, as the scarcity of this resource diminishes and issues such as energy security and environmental impact are given greater importance, other methods of electricity generation are being sought. Nuclear fusion offers the possibility of large-scale electricity generation with none of the drawbacks associated with $\mathrm{CO}_{2}$ production and fuel scarcity with fossil-fuel based energy sources. Furthermore fusion reactors produce no long-lived radioactive waste, unlike modern nuclear fission power plants. The heart of a nuclear fusion reactor is likely to contain a superheated mixture of Deuterium and Tritium plasmas. The hot core of this plasma is such that the conditions are favourable for energy-releasing reactions to take place, heating the plasma and facilitating electricity generation. The reactions with the greatest likelihood within a thermonuclear reactor are [3]:

$$
\begin{array}{cl}
{ }_{1}^{2} \mathrm{D}+{ }_{1}^{3} \mathrm{~T} \rightarrow{ }_{2}^{4} \mathrm{He}+{ }_{0}^{1} \mathrm{n}+\gamma(17.6 \mathrm{MeV}) & \text { (D-T fusion) } \\
{ }_{1}^{2} \mathrm{D}+{ }_{1}^{2} \mathrm{D} \rightarrow{ }_{2}^{3} \mathrm{He}+{ }_{0}^{1} \mathrm{n}+\gamma(3.27 \mathrm{MeV}) & \text { (D-D fusion) } \\
{ }_{1}^{2} \mathrm{D}+{ }_{1}^{2} \mathrm{D} \rightarrow{ }_{1}^{3} \mathrm{~T}+{ }_{1}^{1} \mathrm{H}+\gamma(4.03 \mathrm{MeV}) & \text { (D-D fusion) } \\
{ }_{1}^{2} \mathrm{D}+{ }_{2}^{3} \mathrm{He} \rightarrow{ }_{2}^{4} \mathrm{He}+{ }_{1}^{1} \mathrm{H}+\gamma(18.3 \mathrm{MeV}) & \text { (D-He } \left.{ }^{3} \text { fusion }\right)
\end{array}
$$

The figure below shows the cross sections for each of the above reactions. The cross-sections for the two D-D fusion reactions are very similar, and the curve shown in the graph is the sum of the two. Given the stronger likelihood of D-T fusion reactions 


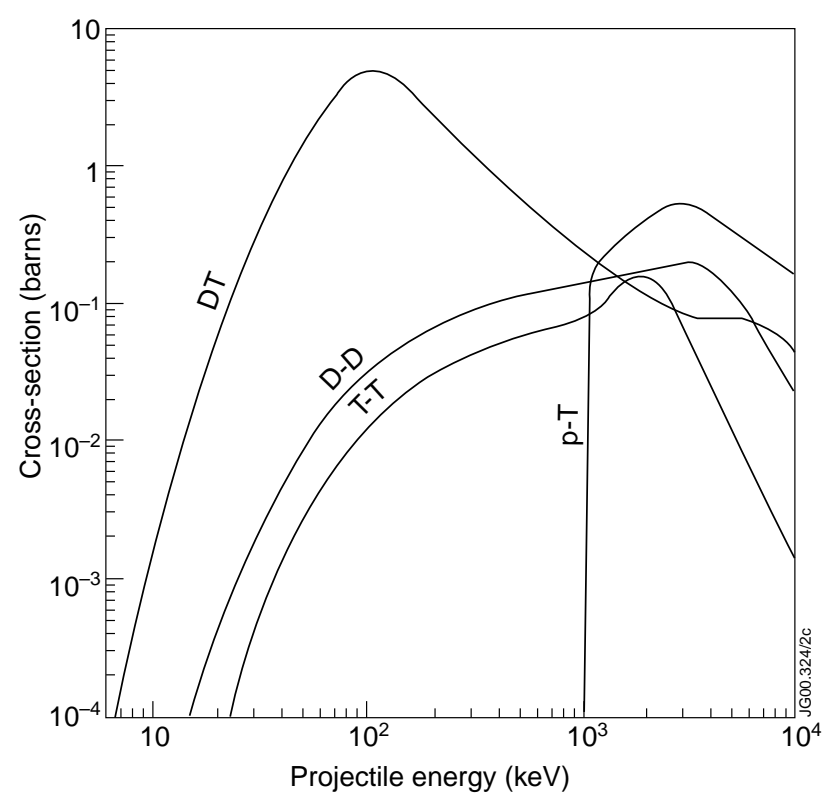

Figure 1.1: Fusion cross-sections as a function of Deuteron energy, courtesy of the JET image database.

occurring at lower Deuteron energy, fusion reactors will exploit this mechanism as the primary source of fusion reactions. Two approaches of exploiting nuclear fusion for energy production are based on inertial and magnetic confinement. In inertial confinement devices, pellets containing a mixture of deuterium and tritium are heated using high energy laser pulses or particle beams to initiate a burning fusion reaction. In magnetic confinement devices an ionised gas, or plasma, is contained within a vacuum vessel housing magnetic field coils which are used to confine the plasma. Several categories of magnetic fusion device exist, including the reversed field pinch, the stellerator and the tokamak. The work in this thesis concentrates on research carried out on a magnetic fusion device based on a variant on the tokamak design known as the spherical tokamak.

\subsection{Introduction to Tokamaks}

The word Tokamak originates from a Russian phrase meaning "toroidal chamber and magnetic coil". As the name implies, the Tokamak was originally a Russian design, which had significant advantages over alternative plasma confinement designs of the day, and became accepted by researchers as a promising route to an eventual fusion reactor.

One of the most prominent features of the Tokamak design are the large toroidal field coils that produce a strong magnetic field "around" the Tokamak. Despite the 


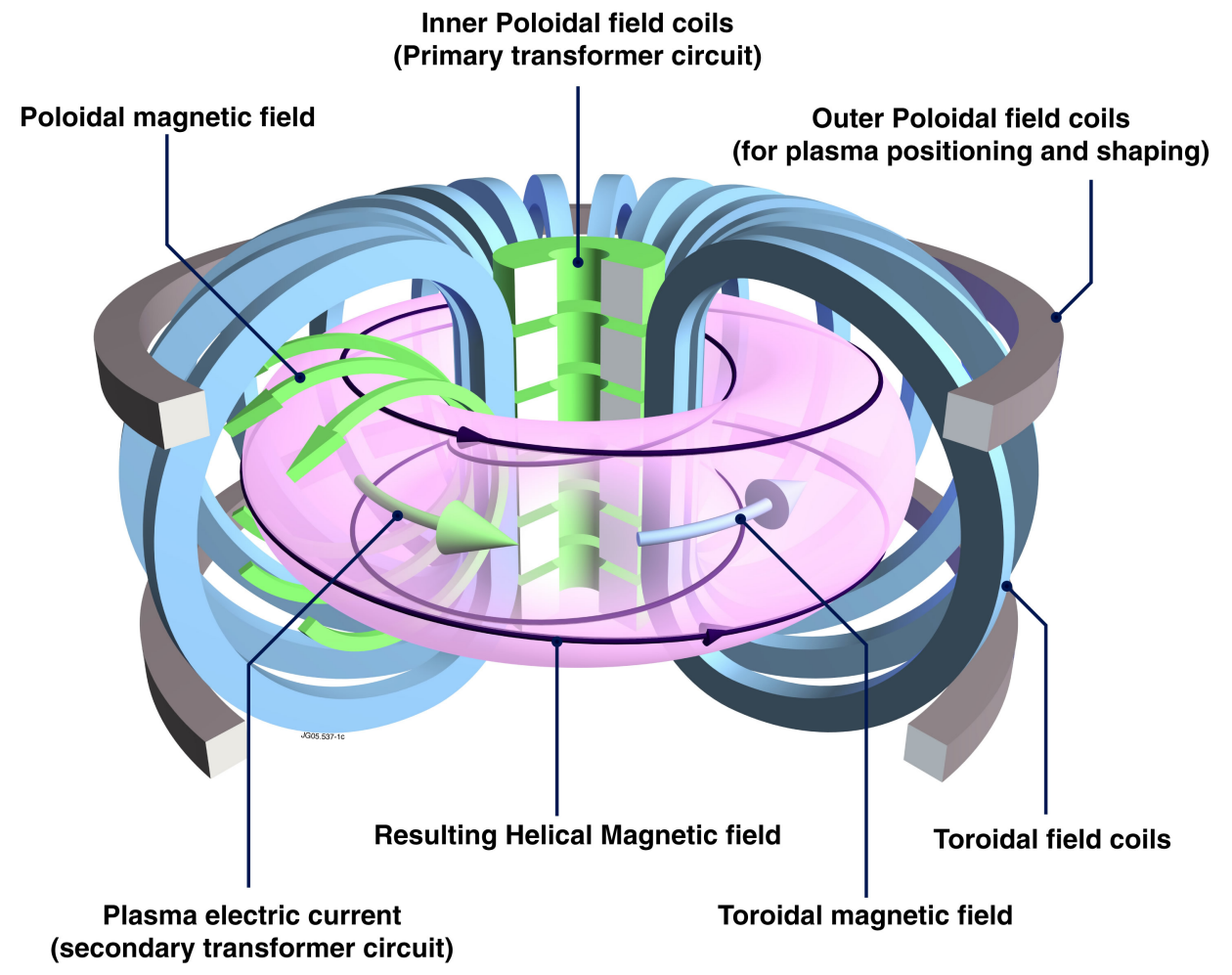

Figure 1.2: Schematic representation of a Tokamak, courtesy of the JET image database.

strong field created by these coils, they alone are not adequate to confine a plasma for a meaningful amount of time. In order to improve plasma confinement, an electrical current is induced within the plasma by the flux from the primary transformer circuit running through the centre of the device. The plasma itself acts as the secondary of the transformer, thus inducing an electrical current. This current has two purposes: firstly, the magnetic field produced by the current, known as the poloidal field, adds a twist to the field lines, which increases plasma confinement significantly. Secondly, the current acts to heat the plasma by ohmic heating, by exploiting its electrical resistance; unfortunately, this effect also results in the plasma current decaying, meaning it must be constantly replenished given a finite plasma resistance. Despite the plasma producing its own poloidal field, external coils are needed to manipulate this field to shape the plasma and to stabilise the plasma in the vertical and horizontal directions.

The Tokamak gained popularity in fusion research circles due to its relatively simple design and good plasma confinement properties. However, interactions between the plasma and the interior surfaces are unavoidable, and can create significant problems for long-pulse or steady-state operation if the consequences of plasma-surface interactions are not mitigated. 


\subsubsection{The Spherical Tokamak}

The spherical Tokamak (ST) is a variation of the original Tokamak design, where the plasma has a more spherical shape, as opposed to the torus shape observed in conventional machines. The first spherical Tokamak, START (Small Tight Aspect Ratio Tokamak) was built at Culham in Oxfordshire with the aim of exploring claims that reducing plasma aspect ratio (the ratio of the plasma major and minor radii, $\mathrm{R} / \mathrm{a}$ ) can have beneficial effects [4]. The experiments carried out on START showed that the ST design has some important advantages in terms of having the ability to operate at a high confinement efficiency, or $\beta$, defined as:

$$
\beta=\frac{\text { plasma pressure }}{\text { magnetic pressure }}=\frac{2 \mu_{0} p}{B^{2}}
$$

The value for $\beta$ is constrained to be below a limit imposed to ensure the plasma is stable against magnetohydrodynamic (MHD) instabilities. A numerical analysis carried out by Sykes and Troyon determined that a maximum value for $\beta$ in a Tokamak can be expressed, roughly, as [5]:

$$
\beta \leq \beta_{\max } \equiv 0.072\left(\frac{1+\kappa^{2}}{2}\right) \epsilon
$$

where $\kappa$ is the plasma elongation and $\epsilon$ is the inverse aspect ratio. Therefore, for a given plasma elongation, a spherical Tokamak plasma is resilient against MHD instabilities at a higher value of $\beta$. This is important as $\beta$ is a measure of the confinement efficiency of a magnetic fusion device, which is related to the cost per unit power of electricity production from a fusion power plant.

Experiments on START have reached average $\beta \approx 40 \%$ [6], which is significantly higher than experimentally observed values on conventional aspect ratio Tokamaks for which $\epsilon$ is large. This remarkable result sparked worldwide interest in the spherical Tokamak design that has persisted to the present and is likely to continue for some time. The successor to START at Culham is the Mega Ampère Spherical Tokamak (MAST) which is currently operating. The engineering specifications of both MAST and START are noted in table 1.1.

The spherical Tokamak concept is being developed with two goals in mind: a component test facility and a spherical Tokamak power plant. Much research needs to be carried out before either of these goals are realised, as research into spherical Tokamaks is in its infancy compared to that of conventional aspect ratio devices. 


\begin{tabular}{|c|c|c|}
\hline & START & MAST \\
\hline Major Radius (m) & 0.32 & 0.7 \\
\hline Minor Radius (m) & 0.25 & 0.5 \\
\hline Elongation & $\leq 4$ & $\leq 3$ \\
\hline Aspect Ratio & $\geq 1.2$ & $\geq 1.3$ \\
\hline Plasma Current (MA) & $\leq 0.31$ & $\leq 2$ \\
\hline Toroidal Field (T) & $\leq 0.5$ & $\leq 0.63$ \\
\hline NBI Heating Power (MW) & 1 & 5 \\
\hline Pulse Length (s) & $\leq 0.06$ & $\leq 1$ \\
\hline Plasma Volume $\left(\mathrm{m}^{3}\right)$ & 0.5 & 8 \\
\hline
\end{tabular}

Table 1.1: Engineering parameters of START and MAST Tokamaks.

\subsection{Plasma-Wall Interactions}

When a plasma and a solid surface meet, plasma-surface interactions (PSI) are the inevitable outcome. The term plasma-surface interactions covers a wide array of behaviour, from physical and chemical sputtering, trapping of fuel atoms within material surfaces (retention), deposition and fuel recycling. The repercussions of PSI are widespread, having a significant effect on the performance and feasibility of a fusion power source. Tokamaks take means to ensure that the effects of these interactions are localised to structures designed to withstand their effects and mitigate their impact on the hot plasma core. The two most popular approaches are limiters and divertors, which will be discussed further in the following section.

\subsubsection{Limiter and Divertor Configurations}

In a simplified magnetic fusion device, such as that shown in figure 1.2, a plasma contained within a vacuum vessel would, come into contact with the inner wall of the device due to cross-field transport mechanisms. This contact between plasma and surface results in the release (sputtering) of atoms that make up the wall into the plasma, thereby degrading the purity of the plasma. Accumulation of impurities has detrimental effects such as fuel dilution and an increased quantity of the energy stored within the plasma being emitted as radiation, having a cooling effect.

An early means of minimising plasma-surface interactions was to have an element protrude radially outward from the wall into the plasma, thereby significantly reducing the plasma flux to the wall of the vacuum vessel. Such an element is known as a limiter. Limiters have advantages in terms of mechanical simplicity and limiting the plasma flux to the walls of the vacuum vessel, but they also have some significant drawbacks.

A limiter effectively dictates the radial extent of the plasma by using an object in contact with the plasma. A region radially outboard of the surface of the limiter, known as the scrape-off layer (SOL) is created by cross-field transport. By assuming 


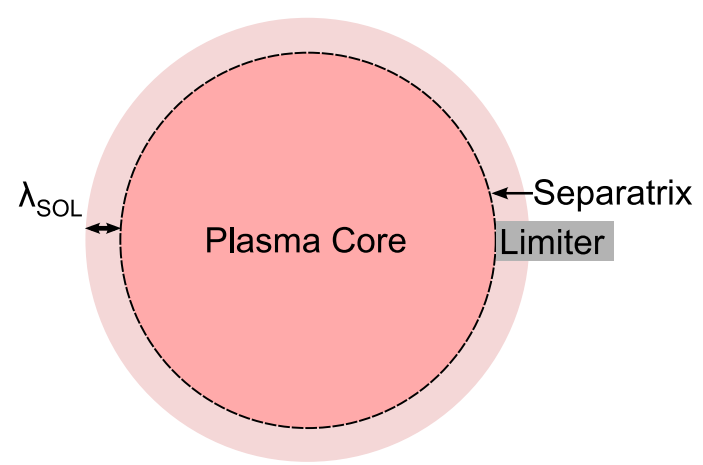

Figure 1.3: Schematic of a Tokamak limiter

that the sole cross-field transport mechanism is due to diffusive processes, the radial extent of the SOL is given by:

$$
\lambda_{S O L}=\left(\frac{D_{\perp} L_{\|}}{c_{s}}\right)^{1 / 2}
$$

where $D_{\perp}$ is the radial diffusion coefficient, $\mathrm{L}_{\|}$is half the distance along an open field line between where it intersects material surfaces and $c_{s}$ is the plasma sound speed, calculated using $c_{s}=\sqrt{2 T / m_{i}}$, where $\mathrm{T}$ is the plasma ion or electron temperature, which are assumed to be equal here. For example, if $\mathrm{T}=25 \mathrm{eV}, \mathrm{L}=20 \mathrm{~m}$, $D_{\perp}=1 \mathrm{~m}^{2} s^{-1}, \lambda_{S O L} \approx 2.3 \mathrm{~cm}$. The relative thinness of the scrape-off layer leads to large heat and particle fluxes on the surface of the limiter, potentially resulting in significant sputtering. As the limiter is in direct contact with the plasma, this can lead to significant impurity accumulation in the main plasma.

The divertor concept overcomes the main drawback associated with limiters associated with direct contact between the primary surface designed to accept heat and particle fluxes in the scrape-off layer. In most Tokamaks with a divertor, this is achieved by diverting the poloidal magnetic field so as to allow the region with intense plasmasurface interactions to be localised in a region away from the core plasma.

A divertor is created by altering the magnetic field of the plasma such as to create a "null" in the poloidal field and creating a region where plasma radially outward of the last closed flux surface is directed into a region away from the core plasma. This configuration has numerous advantages over limiters, such as reducing plasma contamination, increasing the connection length with the poloidal field null, access to the high-confinement mode (H-mode) and an operating regime which can significantly reduce the particle and power flux to plasma-facing materials - detachment. Although there have been signs of detachment behaviour taking place in Tokamaks with limiters, the positive effects of detachment are generally observed in Tokamaks with divertors. 


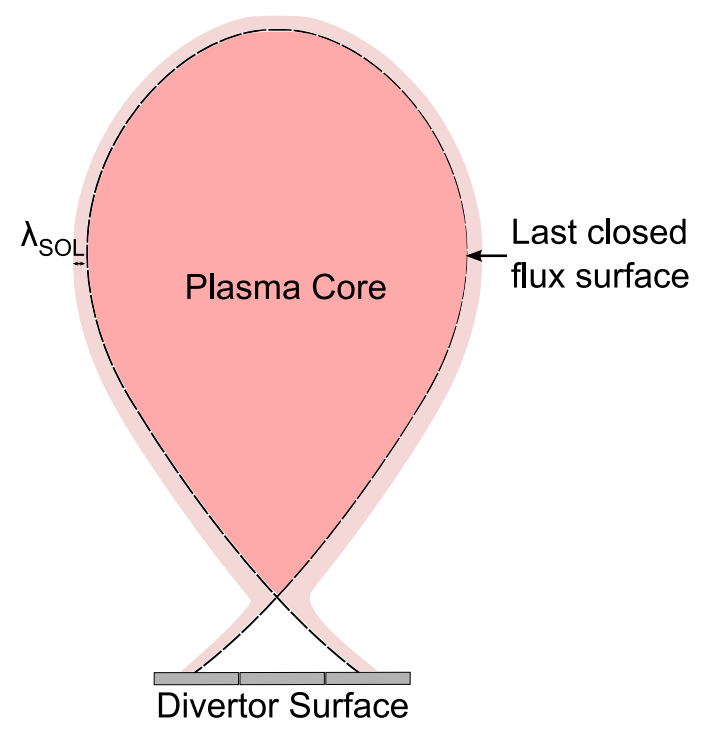

Figure 1.4: Schematic of a Tokamak divertor

\subsubsection{Divertor Detachment}

One of the principal advantages of the Tokamak divertor configuration is to allow access to the so-called detached regime [7]. In a detached divertor scenario, the heat flux to material surfaces in direct contact with the plasma decreases as the particle flux to the surface increases. Precisely why this occurs is unclear, but previous research on diverted Tokamaks have shown that two processes play a role: plasma recombination and ion-neutral friction [8].

When a plasma ion impacts on a solid surface, the surface will be imparted with energy from the ion, resulting in sputtering, but also the ion will recombine with electrons on the surface of the material to form an electrically neutral atom. This process is known as recycling. The newly-born atom is then free to move throughout the Tokamak, unimpeded by the presence of the electromagnetic fields used to confine the plasma. When the atom comes into contact with the plasma, it is ionised to form the ion and electron. In doing so, the plasma expends some energy, the ionisation energy of the atom, in carrying out this process. This has the effect of cooling the plasma. If many such interactions occur between a plasma and a flux of incident neutral atoms, it can lead to the formation of temperature gradients in the plasma. The result of this is that the plasma adjacent to the plasma core is hotter than that close to the divertor, due to proximity to surfaces where recycling takes place.

As the flux of plasma ions to a surface is steadily increased, an increasing number of neutral atoms are created due to recycling processes. Interactions (collisions) with these atoms and their ionisation removes momentum and energy from the plasma, 
ultimately slowing and cooling the plasma reaching the solid surface. Therefore, it seems possible that with enough recycling and ionisation processes taking place over a confined volume, that the plasma could cool to such a degree that it becomes too cold to be a plasma, and cool ions away from solid surfaces begin to recombine with ambient electrons to become neutral atoms. This is an extremely simplified description of divertor detachment - where a plasma incident on a solid surface cools to such a degree that the particles incident on solid surfaces are mostly no longer plasma ions, but neutral atoms. This has the effect of increasing the area of interaction between energetic particles and solid surfaces, as neutral atoms are not constrained to follow magnetic field lines. In addition, increasing quantities of energy is dissipated as radiation as plasma ions recombine to form atoms.

Detachment is critical for the operation of next-step fusion devices such as ITER to moderate the steady-state heat loading to plasma-facing components in the divertor. Extrapolating from existing fusion devices, the peak power loading in the ITER divertor is predicted to exceed $20 \mathrm{MWm}^{-2}$ in the absence of any power moderation mechanism such as impurity seeding or detachment [9]. This is twice the engineering limit of the steady-state heat flux the divertor plates are designed to withstand. As a result, the ITER baseline divertor design assumes that the peak steady-state heat load to the divertor plates will be reduced to approximately $5 \mathrm{MWm}^{-2}$. This requirement will be met using a combination of impurity seeding and divertor detachment.

This thesis will concentrate on measurements taken on the MAST Tokamak where part of the divertor is in the detached state. Interpretation of these measurements is assisted by an Onion-Skin Model (OSM) code, which has been enhanced with a more stable numerical solver and a more developed physics model. The chapters that follow will give a brief overview of divertor physics, the MAST Tokamak and experimental investigations on detachment carried out there, and finally code assisted interpretation of the data collected using OSM and a summary of the results yielded by this study.

\subsection{Thesis Summary}

In chapter 2, a general overview of pertinent aspects of divertor physics is presented, together with a simple analytic model of the tokamak scrape-off layer. Emphasis is placed on divertor operating regimes and the transition to, and characteristics of, the so-called "detached" phase, a principal subject of this study.

Chapter 3 explains some of the pertinent features of the MAST Tokamak to this study in greater detail. This is followed by an overview of the principal diagnostics used, including modifications to the diagnostic hardware and/or data analysis procedures created as a result of this work. 
Chapter 4 contains details of modifications carried out to the OSM transport equations such as terms arising from magnetic flux expansion, parallel viscosity and crossfield drift motion in order to explain a pressure drop routinely observed between the outboard midplane and the lower outer strike point. This chapter also outlines a study into accurate calculation of electrostatic fields supported by the plasma, including the calculation of parallel and cross-field currents and their influence on plasma transport via the $\vec{E} \times \vec{B}$ drift.

Chapter 5 summarises work carried out to characterise a partially detached discharge on MAST using a wide array of plasma diagnostics including Thomson scattering, Langmuir probes, line-of-sight and narrow bandwidth imaging spectroscopy. Checks for consistency within the data set are outlined, together with explanations for inconsistencies when they arise between data from different diagnostics. Finally, the data collected is input into the interpretive OSM-EIRENE code to simulate the ratio of $\mathrm{D}_{\gamma}$ and $\mathrm{D}_{\alpha}$ line intensities and are compared with experimental observations.

Chapter 6 outlines the main conclusions of this study and suggests future avenues of investigation to continue this work.

Appendix A describes the numerical schemes developed as part of this study to facilitate development of the conservation equations used in the OSM plasma transport model, and to allow calculation of plasma potential profiles and electric fields. 


\section{Chapter 2}

\section{Divertor Physics}

In this chapter, an overview of some theoretical aspects of divertor physics is presented including the effects of divertor geometry on the scrape-off layer (SOL), divertor operating regimes and how plasma in the SOL is simulated using modelling codes.

\subsection{Divertor Geometry}

As with many engineering concepts, there exists some considerable diversity in the design of Tokamak divertors. Often, differences exist due to advances in our understanding of plasma behaviour in divertors of other machines, or it can be motivated by research objectives, such as maximising access to diagnostics. Broadly, divertor designs can be ascribed to fall into one of two categories, either open or closed (figure 2.1).

If a divertor is closed, it has a greater capability to capture neutral atoms created by recycling processes taking place at the surfaces of the divertor, and trap the majority of them within a defined volume. Such a design can also offer advantages in terms of ensuring that fewer impurities created in the divertor escape with the possibility of contaminating the plasma core. Also, access to the detached divertor regime requires a lower ion flux to divertor surfaces because of divertor closure. This has the effect of requiring a lower particle density in the plasma core to attain the detached state [10]. Pumping efficiency of impurities and recycled atoms is improved within a closed divertor, as the pressures of theses species increases in a confined volume. One drawback, however, is that by closing a divertor, diagnostic access, especially to imaging systems, reduces. This has the effect of imposing difficulties on researchers who wish to understand the behaviour of plasmas in closed divertor geometries, as structural components can often impede lines of sight across the divertor region.

Conversely, open divertors have fewer structural components to contain neutral atoms and impurities, and therefore can permit significantly greater diagnostic access. For plasma physics researchers examining the divertor, this can be a great advantage; 
however, this comes at the cost of being less able to contain impurities and attain the state of divertor detachment.
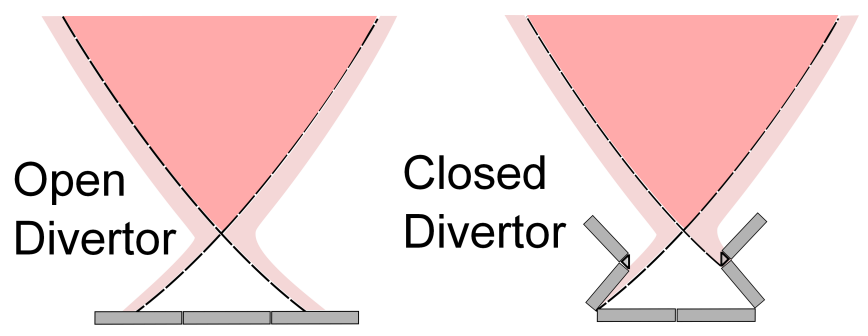

Figure 2.1: Simplified examples of open (left) and closed (right) divertor designs.

The performance of a divertor in terms of trapping created impurities and neutrals with the divertor is largely due to the degree of divertor closure. However, access to the detached divertor state is not a simple function of closure, and has a more subtle relationship with the geometry of the divertor as a whole.

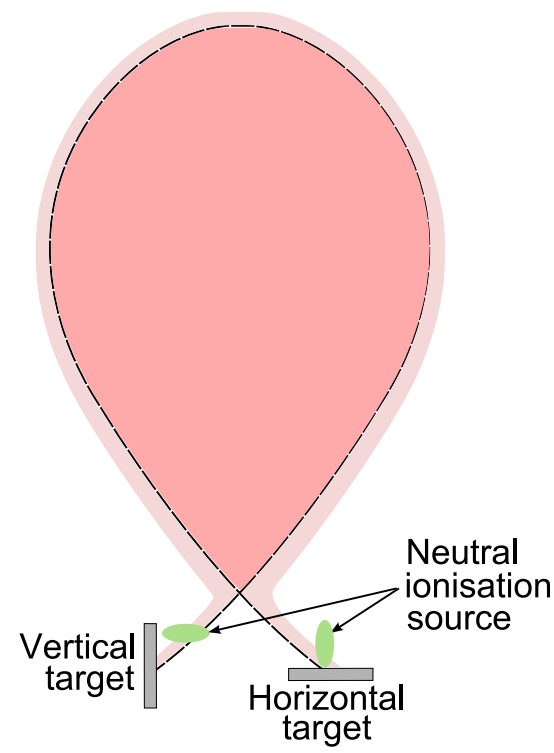

Figure 2.2: Distribution in the particle source, and energy sink, due to the ionisation of recycled neutrals from horizontal and vertical divertor target plates.

An important consideration is also the angle of inclination of the divertor target plates to magnetic field lines. It has been observed that the majority of recycled neutrals depart a divertor surface with a trajectory parallel to the surface normal [11]. The inclination of the target plate with respect to magnetic field lines is important therefore in determining the likely location of where the neutrals are re-ionised in the 
plasma. Figure 2.2 describes the different likely locations where neutrals are to be reionised from horizontal and vertical target plates. Neutrals created on a vertical target are likely to have a horizontal trajectory, passing through the magnetic separatrix, along which much of the heat and particles is transported. The ionisation of neutrals in the vicinity of the separatrix will have a cooling and fuelling effect, raising the plasma density and reducing it's temperature. Such conditions are beneficial for reaching the detached divertor state. Experiments conducted on Alcator C-Mod confirmed that the core density required to detach the plasma at the magnetic separatrix was reduced by $40 \%$ by changing the divertor target inclination from horizontal to vertical [10]. Conversely, neutrals created from horizontal surfaces are likely to undergo a vertical trajectory, resulting in their ionisation taking place away from the magnetic separatrix. As a result, the cooling and fuelling effects of neutral ionisation will take place on field lines that transport plasma with less power and fewer particles. This leads to more heat being transported to the target, and more impurities created by erosion of the divertor surface.

It must be noted, however, that although a vertically inclined target has been found to promote divertor detachment of field lines close to the magnetic separatrix (so-called "partial detachment") a less profound effect is observed with respect to detachment of the whole plasma in the divertor region. On ASDEX-Upgrade, the density limit, which has been shown to be correlated with uncontrolled divertor detachment [12,13], was shown to be unchanged after the Div I divertor was replaced with the Div II, which offered a greater degree of closure [12]. Similar behaviour has also been observed during the evolution of the JET divertor [14].

\subsection{Sheath Physics and the Plasma/Surface Boundary}

The transport of plasma ions toward a solid surface, whether in a divertor or elsewhere, is dominated by the presence of an electrostatic sheath which has profound implications on how particles, momentum and energy are transported from a plasma to a solid surface [15]. The sheath arises due to the higher thermal velocity of electrons compared to ions (by a factor of $\sqrt{\frac{m_{i}}{m_{e}}}$. As a result, a buildup of negative charge accumulates on the surface, resulting in an electric field, known as the ambipolar electric field, that accelerates ions toward it and repels electrons in order to maintain quasi-neutrality. The net result of this field is to equalise the fluxes of ions and electrons to the surface, and hence a detailed understanding of sheath physics is critical in understanding how plasmas and solid surfaces interact.

An important theoretical result, initially derived by Bohm, helps shed light on the nature of particle transport within the sheath, known as the Bohm criterion [16]. A simplified derivation of this result follows. Take a plasma in a 1-D system that contains 
cold stationary ions, i.e. $T_{i}=0$. Within the sheath, there exists a region of net positive charge, due to an attractive negative potential brought about by an accumulation of electrons at the surface, see figure 2.3.

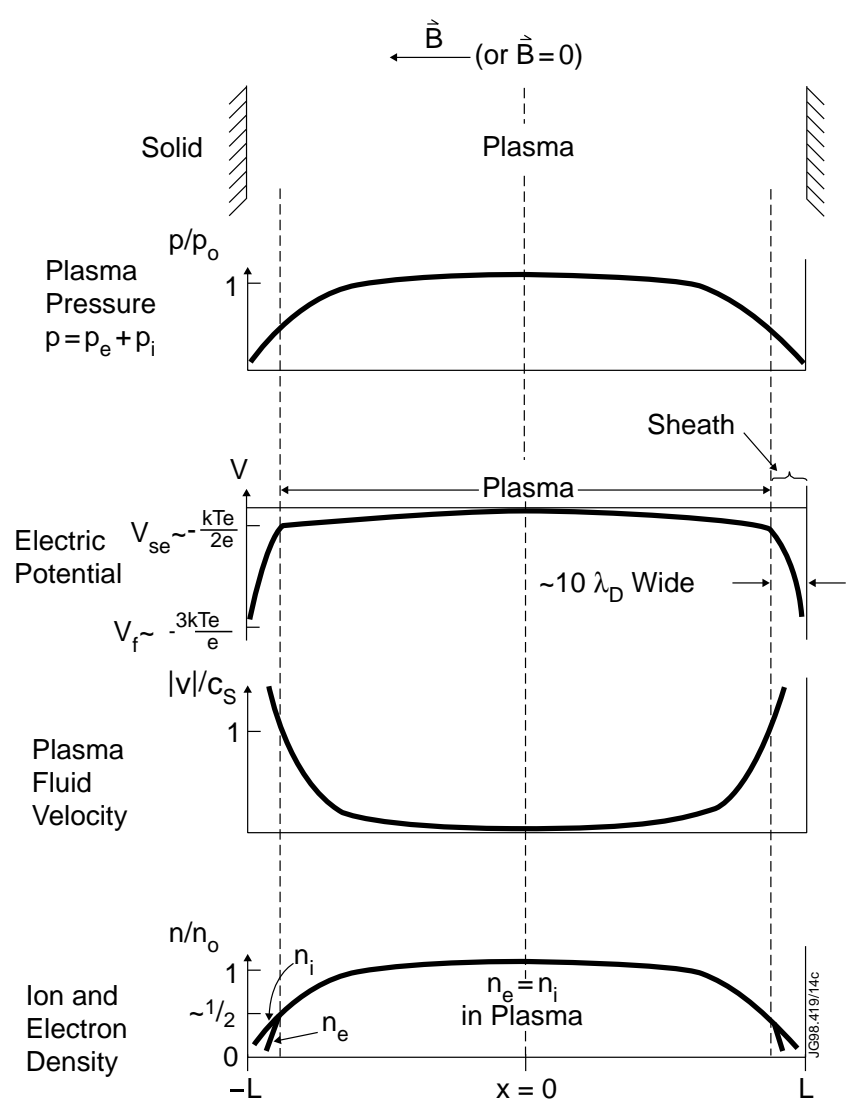

Figure 2.3: System description in a simplified derivation of the Bohm criterion, curtesty of the JET image database.

It can be assumed, that the electrons are approximately Maxwellian, as they exist within a confining potential. Therefore, the electron density within the sheath can be given by a simple Boltzmann relation:

$$
n_{e}(x)=n_{s e} e^{\frac{e\left(\phi-\phi_{s e}\right)}{k T_{e}}}
$$

where $\phi_{\text {se }}$ refers to the electrostatic potential at the sheath edge. Using a statement of conservation of energy, the following expression can be made:

$$
\frac{1}{2} m_{i} v_{s e}^{2}=-e \Delta \phi_{\text {pre-sheath }}=-e \phi_{S e}
$$

This result can be combined with a statement of particle conservation

$$
n_{i} v_{i}=\text { constant }
$$


and Poisson's equation:

$$
\nabla^{2} \phi=-\frac{e}{\epsilon_{0}}\left(n_{i}-n_{e}\right)
$$

to give

$$
\nabla^{2} \phi=-\frac{e}{\epsilon_{0}} n_{s e}\left[\left(\frac{\phi_{s e}}{\phi}\right)^{\frac{1}{2}}-e^{\frac{e\left(\phi-\phi_{s e}\right)}{k T_{e}}}\right]
$$

if a parameter $\Phi$ is defined:

$$
\Phi=\phi_{s e}-\phi
$$

where $\Phi$ is taken to be positive, the following expansions can be made:

$$
\begin{gathered}
\left(\frac{\phi_{s e}}{\phi}\right)^{\frac{1}{2}} \approx 1+\frac{1}{2} \frac{\Phi}{\phi_{s e}}=1-\frac{1}{2} \frac{\Phi}{\left|\phi_{s e}\right|} \\
e^{\frac{e\left(\phi-\phi_{s e}\right)}{k T_{e}}} \approx 1-\frac{e \Phi}{k T_{e}}
\end{gathered}
$$

yielding

$$
\nabla^{2} \Phi \approx \frac{e n_{s e} \Phi}{\epsilon_{0}}\left(\frac{e}{k T_{e}}-\frac{1}{2\left|\phi_{s e}\right|}\right)
$$

from the above equation, some important properties of the sheath can be determined. Firstly, given the solution to (2.9) should be non-oscillatory, the following constraint exists:

$$
\begin{gathered}
\frac{e}{k T_{e}} \geq \frac{1}{2\left|\phi_{s e}\right|} \\
\frac{1}{k T_{e}} \geq \frac{1}{m_{i} v_{i}^{2}} \\
v_{s e} \geq \sqrt{\frac{k T_{e}}{m_{i}}}=c_{s}
\end{gathered}
$$

Therefore, ions exiting the sheath must do so at a speed greater than or equal to the plasma sound speed, $c_{s}$. Furthermore, the length scale of the sheath, $L_{s}$, can be derived by examining (2.9) and making a further simplification [8]:

$$
\frac{\Phi}{L_{s}^{2}} \approx \frac{e n \Phi}{\epsilon_{0}} \frac{e}{k T_{e}}
$$




$$
L_{s} \approx\left(\frac{\epsilon_{0} k T_{e}}{e^{2} n}\right)^{\frac{1}{2}}
$$

where the term on the right-hand side is known as the Debye length. If $T_{e}$ is taken to be $30 \mathrm{eV}$ and $n$ as $1 \times 10^{19} \mathrm{~m}^{-3}, L_{s} \approx 13 \mu \mathrm{m}$. This gives an indication that the sheath region is very thin compared to the length scales ions traverse from a Tokamak midplane to a divertor surface, which is normally 10 's of metres.

The final part of this section concentrates on how the electrostatic sheath affects heat transport. When considering electrons in the sheath, there is a flux of electrons toward the solid surface that have sufficient energy to overcome the Coulomb barrier created by the positive charge in the sheath. Such electrons must have energy of at least $\left|e \phi_{\text {sheath }}\right|$. Any electrons reflected away from the surface by the sheath do not contribute to a heat flux to the surface. Therefore, it could be surmised that the sheath acts as a filtering mechanism to block the passage of low energy electrons toward the solid surface. The result of this is that the high-energy tail of the electron energy distribution is lost to the surface, effectively "cooling" the electrons.

The energetic electrons lost to the surface contribute to the negative charge on the wall that accelerates ions toward it. Therefore, the energy lost by the electrons is effectively heating the ions. As more ions impinge on the surface, more electrons are required to regenerate the sheath, and so this mechanism acts to constantly transfer energy from electrons to ions. Therefore, the total amount of heat that can be transported across the sheath corresponds to a proportion transported by high-energy electrons able to surmount the sheath potential and ions which are accelerated by the sheath. Overall, the total power flux to a surface can be given by:

$$
q=\gamma_{s} k T_{e} \Gamma_{s e}
$$

where $\Gamma_{s e}$ is the particle flux at the sheath entrance and $\gamma_{s}$ is the sheath heat transmission coefficient, which can be approximated by [8]:

$$
\gamma_{s} \approx \frac{2.5 T_{i}}{T_{e}}+\frac{2}{1-\delta_{e}}-0.5 \ln \left[\left(2 \pi \frac{m_{e}}{m_{i}}\right)\left(1+\frac{T_{i}}{T_{e}}\right)\left(1-\delta_{e}\right)^{-2}\right]
$$

where $\delta_{e}$ is the secondary electron emission coefficient, given by the number of incident electrons to a surface minus the number of reflected or back-scattered electrons [8]. If $\mathrm{T}_{i}=\mathrm{T}_{e}$, which is the case when frequent energy-exchange collisions between ions and electrons take place, and $\delta_{e}=0$ is assumed, $\gamma_{s} \approx 7$. 


\subsection{The Two-Point Model}

A simplified model exists to relate the plasma conditions upstream of the divertor to those at a divertor target, known as the two-point model [8] (as the plasma conditions at only two locations are considered). This simple model can be used to estimate the divertor plasma conditions given those close to the plasma core, which can be useful in planning edge physics experiments. The simplified geometry used in the two-point model is outlined in figure (2.4).

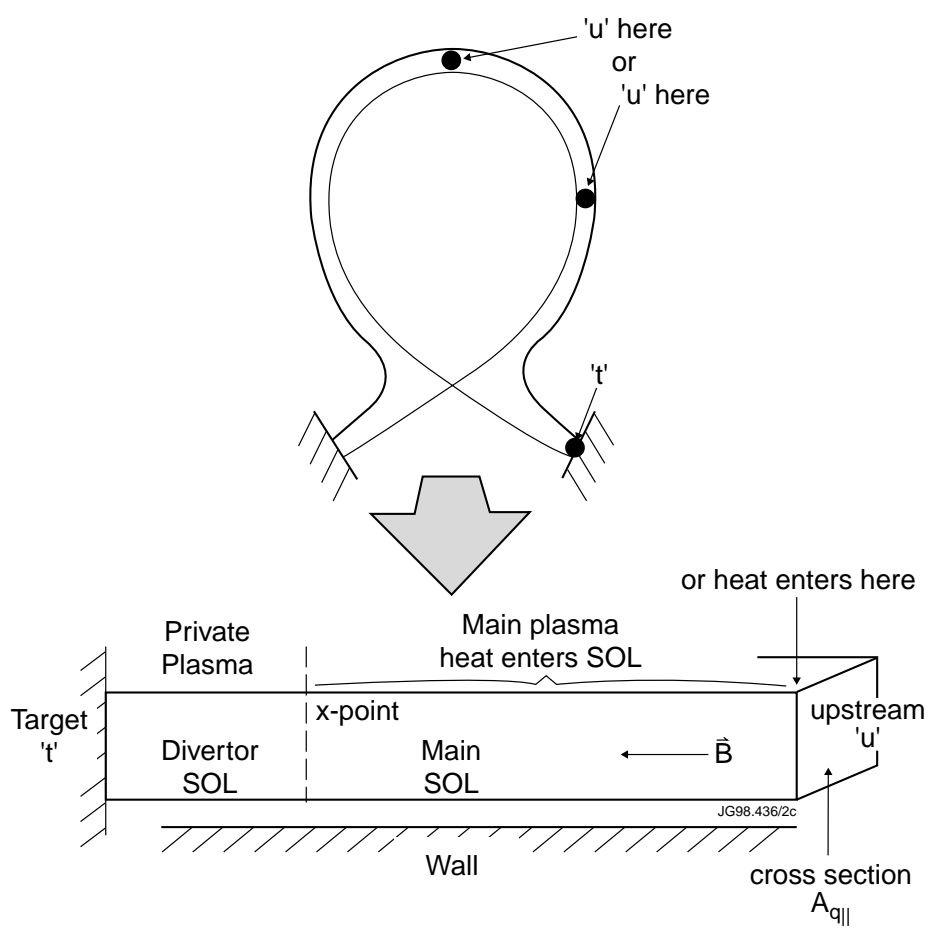

Figure 2.4: Description of the geometry setup in the two-point model. The model is largely insensitive to the "upstream" location, assuming along-field gradients in density and temperature are negligible away from divertor targets. Curtesy of the JET image database.

In the two-point model, magnetic field lines are effectively "straightened out" such that an open field line begins at an upstream location and terminates at some known distance along the line. The location of the upstream position is unimportant assuming that gradients in the quantites of interest are ignorable except in a region adjacent to the divertor targets. The main assumptions of the two point model are:

1. Particle balance for specific field lines is enforced. In other words, cross-field transport is ignored in the model. Furthermore, ions that impinge on a solid surface and recombine to form neutrals are re-ionised on the same field line in a region close to the divertor target. 
2. Plasma flow is assumed to be negligible except in a thin region where ionisation processes take place adjacent to the divertor targets. In this region, the plasma accelerates from stagnation just before the entrance to the ionising region to the plasma sound speed when entering the sheath.

3. Pressure balance is enforced along individual field lines. Effects of viscosity, from any source, are neglected.

4. Power balance is enforced on individual field lines. As the plasma is assumed stagnant over the majority of the field line, parallel heat conduction is assumed to be the sole heat transport mechanism.

5. The only heat source present in the field line is located at the upstream location, and all of this heat is transported to the divertor target.

The above statements can be summarised in the following mathematical statements:

$$
n k T_{e}+n k T_{i}+m_{i} n v_{i}^{2}=\text { constant }
$$

The first two terms in (2.17) define the static pressures of the ion and electron species, the final term defines the dynamic pressure due to the ions. Therefore, assuming that $T_{e}=T_{i}$ and that plasma flow only takes place at the target, the following relationship holds:

$$
2 n_{u} k T_{u}=2 n_{t} k T_{t}+m_{i} n_{t} v_{i, t}^{2}
$$

This expression can be further simplified by incorporating the Bohm criterion, taking $v_{i, t}^{2}=\frac{2 k T}{m_{i}}$, yielding:

$$
2 n_{T} T_{t}=n_{u} T_{u}
$$

Taking the parallel heat conduction density $\left(\mathrm{Wm}^{-2}\right)$ to be:

$$
q_{\|, \text {cond }}=-\kappa_{0} T^{\frac{5}{2}} \frac{d T}{d s}
$$

This term can be integrated with respect to the along-field distance, $\mathrm{s}$, to give:

$$
T(s)=\left[T_{0}^{\frac{7}{2}}-\frac{7}{2} \frac{q_{\|, \text {cond }} L}{\kappa_{0}}\right]^{\frac{2}{7}}
$$

where $T_{0}$ is an integration constant, the plasma temperature at a reference location. Using (2.21), the temperature upstream can be related to that at the divertor target:

$$
T_{u}^{\frac{7}{2}}=T_{t}^{\frac{7}{2}}+\frac{7}{2} q_{\|} \frac{L}{\kappa_{0 e}}
$$


Finally, using the assumption that no power loss mechanisms exist along field lines, the power source upstream must equal the power delivered to the target across the sheath, hence:

$$
q_{\|}=q_{t}=\gamma n_{t} k T_{t} c_{s t}
$$

This completes the equations that make up the two-point model.

$$
\begin{aligned}
2 n_{t} T_{t} & =n_{u} T_{u} \\
T_{u}^{\frac{7}{2}} & =T_{t}^{\frac{7}{2}}+\frac{7}{2} \frac{q_{\|} L}{\kappa_{0 e}} \\
q_{\|} & =\gamma_{s} n_{t} T_{t} c_{s t}
\end{aligned}
$$

where $n_{u}\left(\mathrm{~m}^{-3}\right)$ and $q_{\|}\left(\mathrm{Wm} m^{-2}\right)$ are taken as input, $\gamma_{s} \approx 7$ and $\kappa_{0 e}$ is given by [8]:

$$
\kappa_{0 e}=\frac{30692}{Z_{i} \ln \Lambda} \approx 2000
$$

where $Z_{i}$ is the ion species charge and $\ln \Lambda$ is the Coulomb logarithm, which can be taken as approximately 15-17. $L$ is the connection length, which is half of the along-field distance between two plasma-facing surfaces bounding the field line. The two-point model is now composed of a closed system of three unknowns; $T_{t}, T_{u}$ and $n_{t}$. Of course, this need not be the case, provided enough information is known about the plasma in question to reduce the problem to three unknowns, the two-point model equations can be closed.

\subsection{Divertor Operating Regimes}

\subsubsection{The Sheath-Limited Regime}

At moderate values of upstream density, the plasma in the SOL of a Tokamak can be said to be in the sheath-limited regime. The characteristics of this state are [8]:

1. Parallel temperature gradients of ion and electron species are negligible, i.e. $\nabla_{\|} T_{e, i} \approx 0$.

2. Recycling taking place at the divertor targets takes place at moderate levels, due to low upstream density.

3. The effects of volumetric recombination and ion-neutral friction are so small as to be ignorable. 
4. Electrostatic sheaths are the dominant heat sink for the SOL.

Such conditions lend themselves well to two-point modelling, as many of the assumptions made in the formulations in the model hold in this operating regime. As a result, the sheath-limited regime is also known as the simple scrape-off layer (SOL). The absence of strong recycling taking place at divertor targets and the absence of parallel ion and electron temperature gradients are linked; the ionisation or recycling neutrals, and also charge-exchange interactions, have a cooling effect on ions in the SOL and hence generate the parallel gradients. The lack of parallel temperature gradients is concerning when considering the sheath-limited regime in terms of Tokamak operations. If no (or only very weak) energy sinks exist, such that the temperature of plasma species is a constant along field lines, the heat imparted onto plasma-facing components is mediated only by the electrostatic sheath, hence the name sheath-limited regime. As a result, physical sputtering of plasma-facing components in a fusion reactor will be at its greatest when the SOL is behaving in this way. Therefore, despite the advantages offered by the apparent simplicity of the sheath-limited regime, it is considered to be the least beneficial.

\subsubsection{The Conduction Limited Regime}

The defining feature of the conduction-limited regime is the presence of temperature gradients parallel to magnetic field lines. The sign of these gradients, starting upstream and finishing at the divertor target, are always negative, which has obvious advantages in terms of reducing sputtering of plasma-facing components. In order for these gradients to form, it is normally necessary for parallel heat conduction to dominate over convection as the primary heat transport mechanism in the SOL.

How this situation comes about is best considered by examining the distribution of particle sources in the sheath-limited and conduction-limited regimes. In the sheathlimited regime, the particle source for a given field line in the SOL originates from cross-field transport upstream of the divertor targets. As a result, particle flows are generated in the upstream region, resulting in the majority of the power entering the field line being transported by convection, as is the case in the sheath-limited regime, see figure 2.5. Conversely, if the main particle source were due to ionisation of neutrals close to the divertor surface, the flows in the SOL would be significantly diminished, meaning that conduction becomes the primary heat transport mechanism. A direct result of this change in heat transport mechanism is the necessity for the existence of parallel temperature gradients in order to facilitate heat transport. This alteration in the distribution of the SOL particle source is what is thought to bring about the transition from the sheath-limited to the conduction-limited regime. Note that in both regimes, power enters the SOL upstream of the divertor surface. 

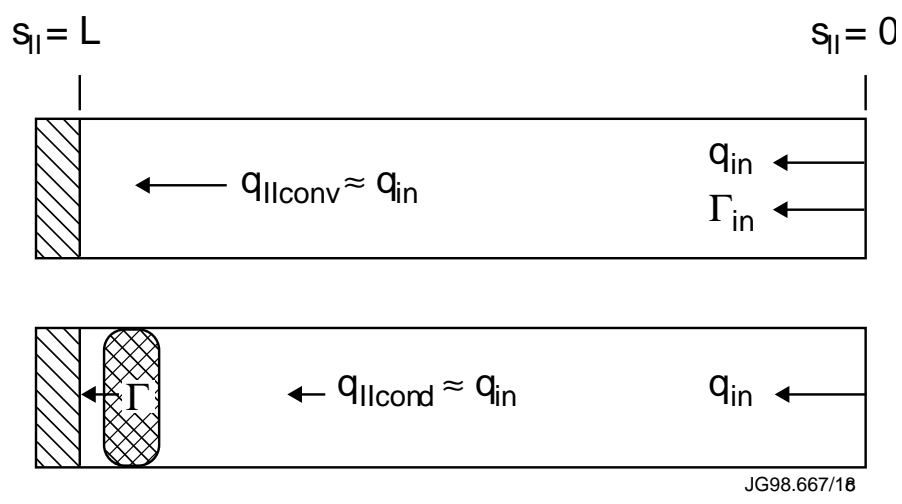

Figure 2.5: Distribution of particle sources in the sheath-limited regime (top) and conduction-limited regime (bottom) [8], courtesy of the JET image database.

During a Tokamak pulse, the particle source close to the divertor surface would be provided by the ionisation of recycled neutrals. As a result, as the recycling rate, which is linked to the particle flux reaching the surface, is linked to the upstream density, it is this parameter which increases to bring about a transition from the sheath-limited to conduction limited regime.

\subsubsection{Detachment}

Divertor detachment, as defined by Matthews [7] is described as the, "state in which large pressure gradients (static plus dynamic) are observed parallel to the magnetic field with consequently low plasma power and ion fluxes to the material surfaces bounding the system." The statement that pressure gradients exist parallel to field lines, at all, creates a clear distinction between the detached and aforementioned states. Clearly, consideration of divertor detachment with a two-point model would require significant corrections in order to account for pressure loss mechanisms. This could lead to the conclusion that a simple analytic model of the SOL might struggle with handling this additional complexity; as it turns out, it also poses significant challenges for sophisticated computer simulations.

However, the definition of detachment goes on to describe the most attractive feature of this operating regime, the reduction in power and ion fluxes to material surfaces. As a result, divertor detachment is an important means of handling the large steady-state heat loads predicted in next-step devices such as ITER. Due to the complexity of the detached state, it will be explored in greater depth in the following section. 


\subsection{Properties of Detached Plasmas}

The presence of divertor detachment is defined [8] by the following observations:

1. An increase of ion saturation current measured at the divertor targets, roughly as $n_{u}^{2}$, followed by a decrease. This is known as the 'roll-over'.

2. $D_{\alpha}$ radiation emitted from the divertor targets continues to increase even when the ion saturation current is decreasing.

Another important characteristic of divertor detachment is a low electron temperature, of the order of $1 \mathrm{eV}$. Measurement of electron temperatures in detached plasmas is normally problematic as standard Langmuir probe analysis methods are not well suited to cold plasmas [8]. As a result, probes tend to provide an overestimate of the electron temperature, meaning that alternative, spectroscopic, methods must be employed [17]. A routine measurement on Tokamaks is the so-called degree of detachment (DoD), which is defined as [18]:

$$
\mathrm{DoD}=\frac{\Gamma_{t}^{\text {calc }}}{\Gamma_{t}^{\text {measured }}}
$$

where $\Gamma_{t}^{\text {measured }}$ and $\Gamma_{t}^{\text {calc }}$ are empirically measured and analytically calculated ion fluxes to divertor target plates, respectively. The reasoning behind this figure of merit is that the ion flux to a divertor target in the conduction-limit regime scales, roughly, as:

$$
\Gamma_{t}^{\text {measured }} \propto n_{u}^{2}
$$

therefore, as a plasma makes the transition from the conduction-limited to the detached regime, the ion flux to the divertor target should initially scale with $n_{u}^{2}$ before reaching a maximum level and roll-over when detachment has been established. This behaviour for a JET discharge is shown in figure 2.6.

Another striking feature of detachment which has already been alluded to is the loss of pressure conservation along magnetic field lines. This is not observed in other divertor operating regimes, which makes it unique to detached plasmas. A simplified representation of divertor detachment is given in figure 2.7, where an ion, emerging in a hot plasma upstream of the divertor, moves parallel to magnetic field lines into the divertor region, which is detached. As a result of detachment, the neutral pressure is high, and so the ion undergoes multiple momentum-loss collisions. In order for neutrals to be able to effectively remove momentum from the plasma, they collide with the walls of the divertor chamber. It is now possible to understand why pressure is no longer conserved along magnetic field lines; in order for neutrals to be able to effectively 


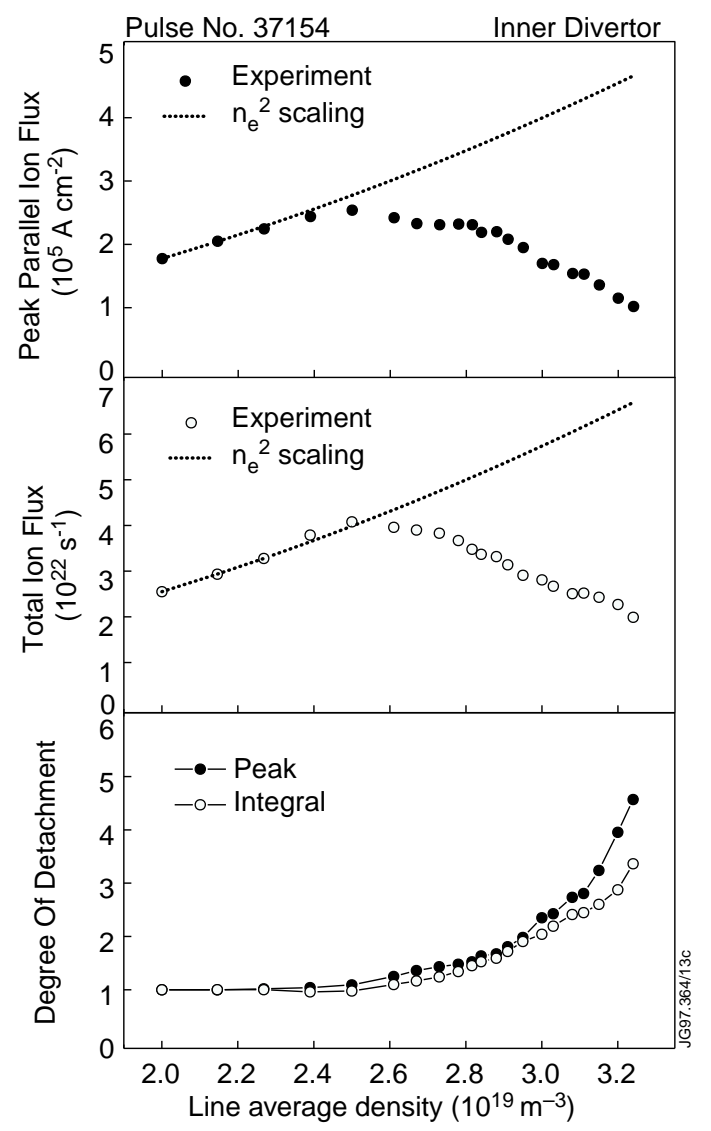

Figure 2.6: Evolution of a discharge on JET. The upstream density was continually ramped throughout the discharge, producing a "rollover" in the ion flux measured at the inner divertor target [18].

remove momentum from the ions, the neutrals must loose their momentum to material surfaces [19].

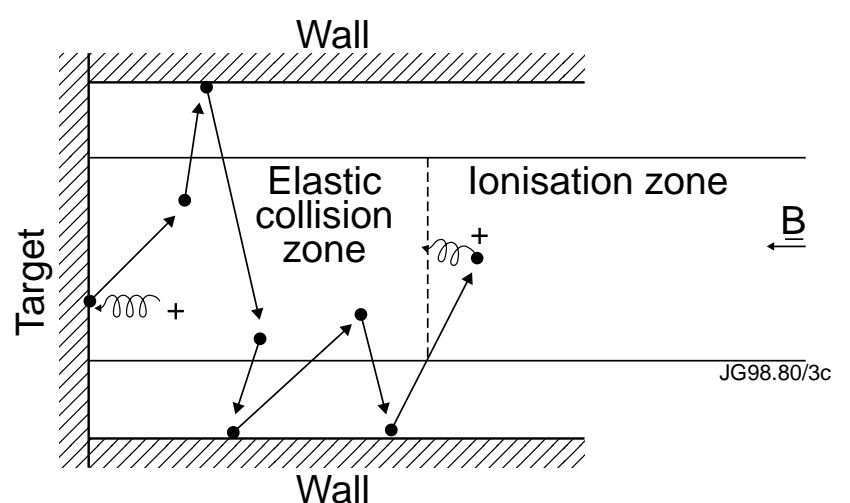

Figure 2.7: Schematic of a simplified description of detachment. An ion emerging upstream in the hot, ionising zone moves into the elastic collision zone and undergoes momentum loss collisions with ambient neutrals [7]. 
A property of detached plasmas that stems from their low temperatures are signs of volumetric ion-electron recombination. This has the effect of lowering the ion and electron densities and increasing the neutral density. Due to this effect, it is another common feature of detached plasmas that as the ion flux to divertor surfaces is decreasing, the neutral pressure increases. An important signature of recombination processes is the presence of high-n Balmer emission lines [20], which can also yield line integrated electron density and temperature measurements.

\subsubsection{Stability of Detachment}

Experiments on many Tokamaks, including JET [18], DIII-D [21], TCV [22] and others have yielded observations of an unstable dense, radiating region known as a MARFE (Multifaceted Asymmetric Radiation From the Edge [23]) in cases of uncontrolled divertor detachment. The presence of a MARFE is widely seen as a precursor to L-mode density-limit disruptions [9], which can be the cause of termination of many fully detached divertor plasmas.

As divertor detachment proceeds, the recombining region expands as the neutral density in the divertor increases, cooling incident ions and electrons to the point where they too recombine. The result of this volumetric recombination is an expanding barrier of neutrals between the plasma and the divertor plate, which, if given sufficient time, can reach the x-point. An example of such a series of events is shown in figure 2.8, taken from [24], where a radiating zone, forming in the divertor, subsequently moves towards the x-point, resulting in a density-limit disruption. This is a large drawback of the detached state: if unimpeded, detachment can lead to an uncontrolled termination that results in a significant transient heat load to plasma-facing components.

Hutchinson [25] carried out an analytic study of detachment and MARFE formation, assuming pressure conservation and analysing the behaviour of the steady-state heat conduction equation. As a result of this study, he notes that the range of densities over which the thermal front remains in the divertor leg is around $20 \%$ in main chamber density from the detachment threshold. Exceeding the density range results in a MARFE localised at the x-point over a larger density range.

Another issue in terms of detachment stability can be seen by examining the opposite extreme: a burst of hot plasma entering a detached divertor leg, such as due to an ELM [26], [27]. The effect of large heat pulses entering the cold plasma region is to ionise the neutrals close to the divertor target, and hence diminishes evidence of detachment, albeit transiently. As a result, the cold plasma region does not remove all incident energy from an ELM and some does reach plasma-facing components. The fraction of ELM energy dissipated is a function of ELM type, degree of detachment and divertor geometry. This does demonstrate, however, that although detachment is an 

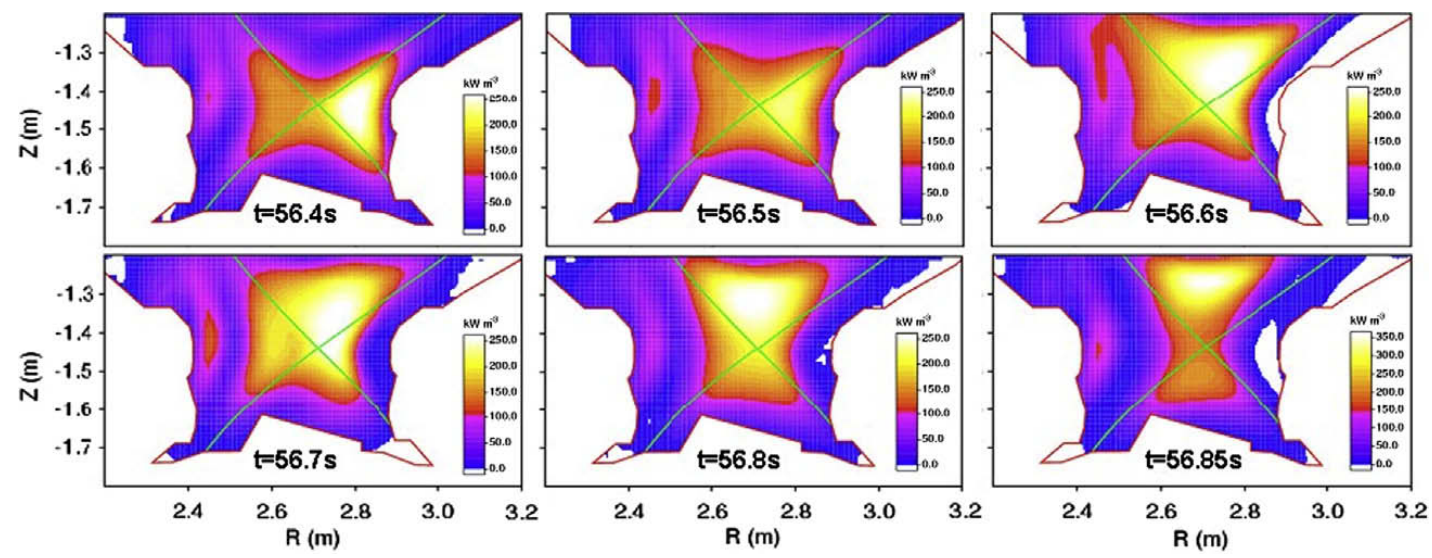

Figure 2.8: Bolometer data taken during a JET discharge. At 56.4s, most of the radiation is concentrated in the divertor region. At subsequent times, the radiating region moves upwards toward the x-point [24].

extremely useful operating regime in terms of reducing the steady-state heat loading, and heat loading between ELMs, of plasma-facing components in a Tokamak, it may not mitigate transient heat loads due to large ELMs.

\subsection{Edge Physics Modelling}

Computer models of Tokamak plasmas can be broadly sub-divided into two categories: interpretive and predictive. Interpretive models are intended to offer greater insight into experimental measurements by pooling the data collected, and using the information to apply boundary conditions and constraints on a physical model. Where a disparity between the model and experimental data exists, extra numerical terms are incorporated in the model to ensure that the plasma simulation converges toward experimental measurements where provided. The reasons for using interpretive models are twofold: to better understand an experiment, and to test the validity of a physical model. An example of an interpretive code applied to the boundary plasma of MAST is outlined in the next section.

Predictive models, on the other hand, consist of a widely diverse group of codes that is far too complex to treat fully in this section. In brief, the majority of predictive models are based on the fluid models that treat the plasma as a $2 \mathrm{D}$ or fully $3 \mathrm{D}$ system. They are intended to provide greater insight into plasma behaviour and experiments by allowing the user to simulate experiments for which no experimental data exists, and so has great appeal. The necessity for such codes arises as design efforts for next-step devices intensify, as interpretive codes, by definition, are not intended to study plasmas formed in Tokamaks that do not yet exist. At present, the most widely used predictive edge fluid codes used on Tokamaks are B2-SOLPS [28], EDGE2D [29] and UEDGE [30], 
which include sophisticated numerical schemes that allow the codes to model plasma conditions using complex systems of conservation laws of particles, momentum and energy.

Furthermore, as plasma theory develops and computers become faster, predictive codes are able to capture a wider array of plasma behaviour than was previously possible. Perhaps one of the greatest challenges for predictive codes is the accurate simulation of the mechanisms that drive cross-field transport. At present, it is believed that this transport arises primarily due to a combination of drift motion and turbulence, which significantly complicates efforts to accurately simulate plasma behaviour in the SOL of Tokamaks. However, predictive codes such as BOUT [31] and TOKAM-3D [32] have incorporated turbulence models in a 3D geometry and are providing greater insight into the effects of turbulent motion. Other codes, however, require cross-field transport coefficients to be input by the user, which cannot be known when running a simulation in predictive mode, which can place a limit on the accuracy of the results produced.

\subsection{The Onion-Skin Model}

The Onion-Skin Model (OSM) is based upon the onion-skin method of Stangeby [8], whereby plasma transport is treated along a series of nested magnetic flux tubes, resulting in a $2 \mathrm{D}$ distribution of plasma fluid properties. In this method, parallel (to magnetic field lines) and cross-field transport can be effectively de-coupled from one another, and treated independently of one another. Consequently, this simplification allows the application of simpler 1D transport equations and numerical schemes, as opposed to their more complex $2 \mathrm{D}$ counterparts. Information regarding the $2 \mathrm{D}$ nature of plasma transport is handled in the method by the choice of boundary conditions. In the OSM code, boundary conditions are provided by measurements of plasma properties such as ion flux to target plates, density, temperature, etc, which are normally provided at different flux tubes. In the code used in this study, there is no explicit requirement that the plasma solution be self-consistent, as this relies upon the self-consistency of the experimental data input into the code. This explicit reliance on experimental data means that OSM is an interpretive code, used to provide greater insight into experimental data collected by different diagnostics.

In the original OSM model used in this study, the particle and momentum transport equations are:

$$
\begin{gathered}
\frac{d}{d s}\left(n v_{i}\right)=S_{p} \\
\frac{d}{d s}\left(n e\left(T_{e}+T_{i}\right)+m_{i} n v_{i}^{2}\right)=S_{m}
\end{gathered}
$$



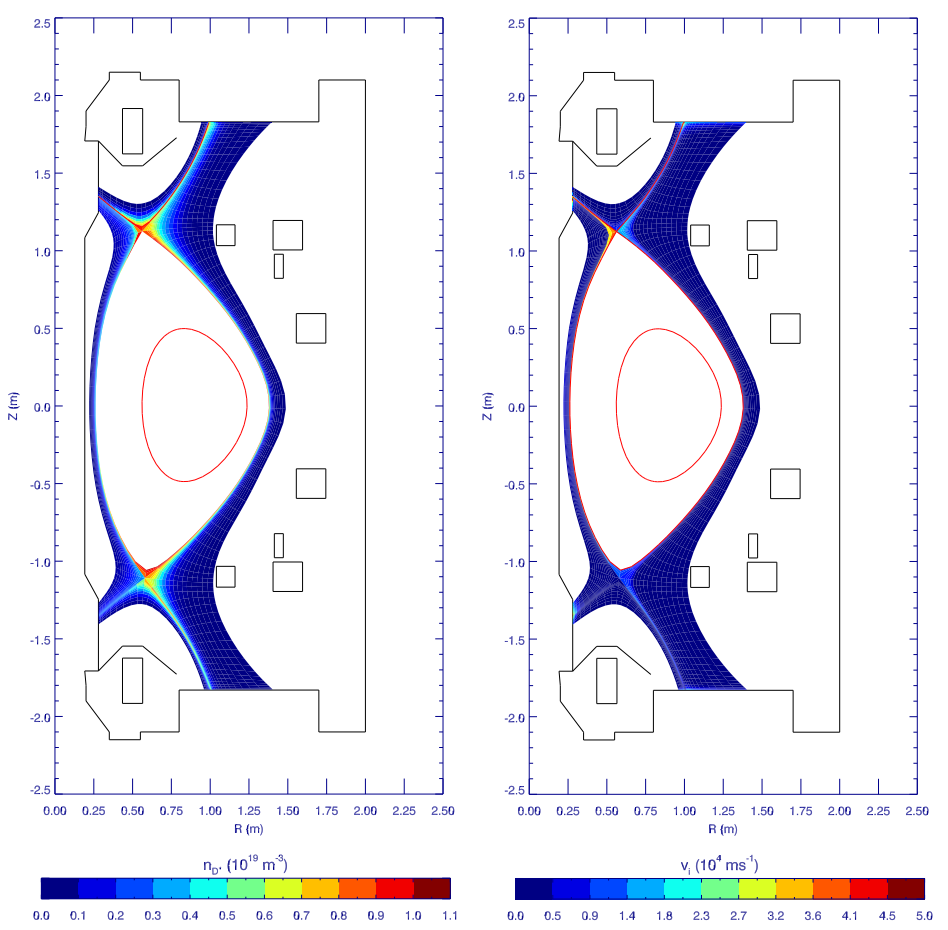

Figure 2.9: Output of an OSM simulation of MAST discharge 13018 at 250ms [33].

in the model, ions have temperature $T_{i}(\mathrm{eV})$ and electrons have temperature $T_{e}$ $(\mathrm{eV})$, the ion and electron species densities are equal, $n$, by quasineutrality ${ }^{1}$ and the parallel ion velocity is $v_{i}\left(\mathrm{~ms}^{-1}\right)$. A relationship between ion and electron temperatures are assumed, $T_{i}=\gamma T_{e}$, where $\gamma$ is taken to be known. Sources (and sinks) of particles and momentum are given by $S_{p}\left(\mathrm{~m}^{-3} \mathrm{~s}^{-1}\right)$ and $S_{m}\left(\mathrm{~kg} \mathrm{~m}^{-2} \mathrm{~s}^{-2}\right)$ respectively. As the transport equations to be solved on each flux tube are two coupled ordinary differential equations, the requirement exists for one boundary condition each for density and parallel ion velocity to be provided to ensure the solution is unique. These boundary conditions are provided by experimental data. Studies carried out comparing an OSM model (OSM2) with a 2D edge transport code EDGE2D [34] showed good agreement between the two codes when carrying out simulations on the same plasma. Figure 2.9 shows an output of a OSM simulation on an ohmically heated MAST discharge, where plasma properties at the divertor plates were determined using Langmuir probes and the plasma density and temperature at the midplane were measured using the MAST Thomson Scattering diagnostic.

\footnotetext{
${ }^{1}$ This is not strictly the case, as quasineutrality requires the difference between the ion and electron densities to be small as opposed to nonzero, but for the present study, this difference it taken to be zero. Furthermore, the presence of ionized impurities results in more electrons than ions being present in the plasma.
} 


\subsection{Summary}

The different divertor operating regimes, sheath-limited, conduction-limited, high recycling and detached, have been briefly reviewed in terms of their effects on plasma transport in a Tokamak SOL. Greater understanding of SOL transport along and across field lines is provided by simulation codes of varying complexity, which often require user-provided information regarding the strengths of cross-field transport mechanisms. Interpretive codes, such as OSM, can be used to deduce SOL transport using data collected from diagnostic data of a given plasma at different flux tubes, which implicitly include information concerning cross-field transport. 


\section{Chapter 3}

\section{The Mega Ampère Spherical Tokamak}

In this chapter, a more detailed description of MAST and magnetic equilibria is given. Descriptions of the relevant diagnostics are described, together with diagnostic equipment and data analysis techniques developed in this study for characterising low temperature, high density plasmas in a Tokamak divertor.

\subsection{Introduction to MAST}

The MAST machine [35] comprises a stainless steel vacuum vessel, intended to sustain a high-vacuum environment within its interior and to support large magnetic field coils and to facilitate diagnostic access to the plasma (figure 3.1). The toroidal field is generated by 24 external coils that subtend from the top and bottom of the machine, extend radially beyond the machine's outer wall, and form a closed loop within the centre column. Within the centre column also resides a solenoid, known as P1, through which electrical currents are ramped. The electromotive force generated couples to the plasma and drives a current that heats and confines the plasma by the generation of a poloidal magnetic field.

The divertor magnetic geometry is generated by the divertor, or P2, coil, in which a current is driven in the same direction to the plasma current to oppose the poloidal magnetic field and create a field "null" known as the x-point. The P2 coil is also used to counter some of the effects of the solenoid fringing field and to control the position of greatest plasma-surface interaction known as the strike point. The induction P3 coil is used to allow an induction-compression scheme of initiating a plasma to reduce the flux required from P1 during the start-up phase of a discharge. The P4, P5 and P6 coils are used for plasma shape control, to counter the "hoop force" [36] pushing the plasma out to larger radial positions due to the plasma current and to maintain vertical 
stability (in the case of P6).

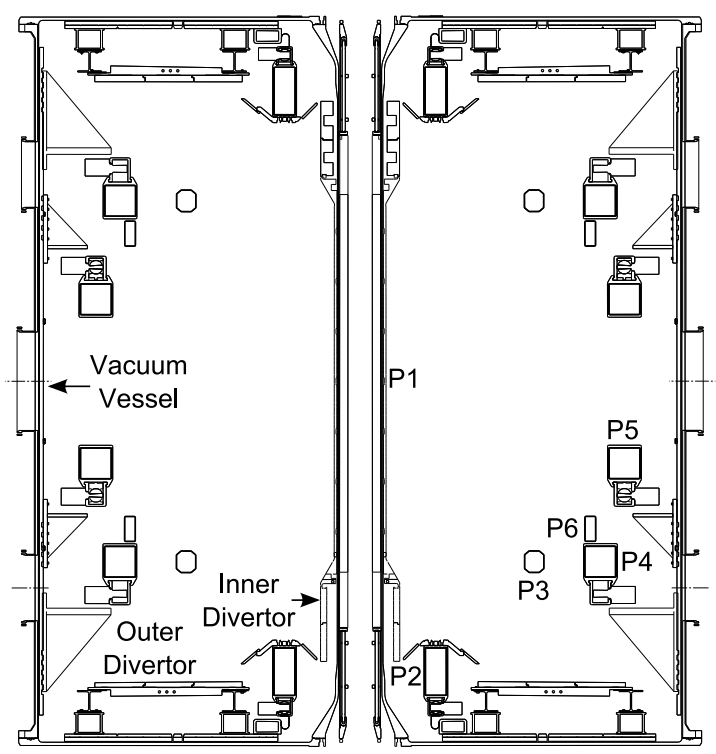

Figure 3.1: Engineering schematic of the MAST vessel, courtesy of the MAST design office.

A typical plasma pulse on MAST lasts for $0.5-0.6 \mathrm{~s}$, which is limited by the finite inductive flux that can be generated by ramping current in $\mathrm{P} 1$, magnetohydrodynamic (MHD) and vertical instabilities.

\subsection{Edge \& Divertor Physics on MAST}

\subsubsection{The MAST Divertor}

The divertor is of an open configuration (figure 2.1), composed of 96 discrete tiles made up of fine-grain EK98 graphite [37]. Carbon-based plasma-facing components are common in Tokamaks due to their low Z (and hence low radiated power in the plasma core), thermal properties and sublimate rather than melt when exposed to strong heat loads. The divertor geometry facilitates significant diagnostics, particularly optical diagnostics that require unimpeded lines of sight from viewing windows mounted on the vessel wall to the divertor plasma.

\subsubsection{Plasma Equilibria}

The geometry of MAST plasmas broadly fall into three categories: upper single-null, where the primary $\mathrm{x}$-point is close to the upper divertor, lower single-null, where the 
primary x-point (magnetic flux surface closed to the magnetic axis of the machine) is close to the lower divertor, and double-null (figure 3.2). Distinction between these equilibria is made using a parameter $\delta \mathrm{R}_{\text {sep }}[38]$, which is the distance between the two magnetic separatrices as measured at the mid-plane at the low-field side, where $\delta \mathrm{R}_{\text {sep }}>0$ denote the primary separatrix being the upper.

Despite the apparent symmetry in the layout of MAST's structural components, aspects of plasma physics such as the Shafranov shift, $\nabla \mathrm{B}$ and other drifts, plasma properties at the divertor strike points display a large asymmetry. In MAST, the ratio of the power flux to the outer and inner targets is typically around 20-40 in connected double-null discharges, where $\delta \mathrm{R}_{\text {sep }} \approx 0[39,40]$. In highly disconnected double-null discharges, where the $\mathrm{x}$-points lie on different flux surfaces, $\delta \mathrm{R}_{\text {sep }} \neq 0$, this ratio falls to approximately 4. An asymmetry exists in the power arriving at the upper and lower divertor targets due to the $\nabla \mathrm{B}$ drift, which under normal configuration is directed downwards [40].
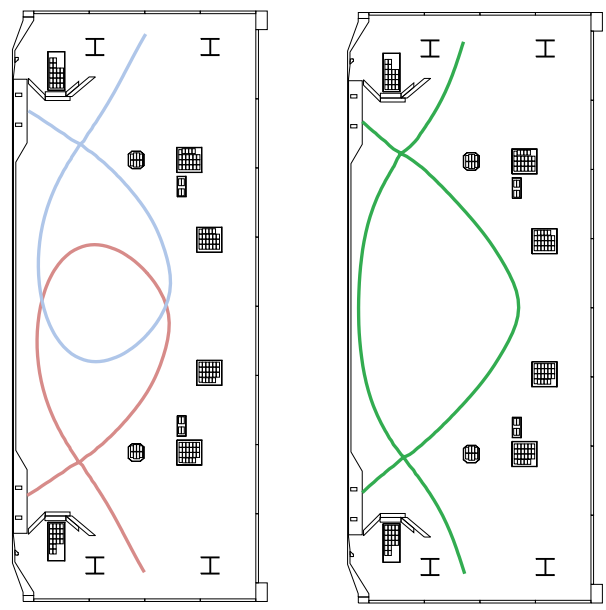

Figure 3.2: Three broad categories of MAST magnetic equilibria exist, lower and upper single-null (left red and blue shapes, respectively) and double null (right shape.)

An overview of the most commonly used terms referring to Tokamak magnetic geometry is given in figure 3.3 .

\subsection{Edge Diagnostics}

\subsubsection{Langmuir Probes}

MAST is equipped with over 700 Langmuir probes embedded in the divertor targets and the centre column for measuring electron temperature, density, ion saturation current and (approximate) power arriving at the probe. Before the MAST Langmuir probe system is explained in greater detail, the principles underlying the operation and 


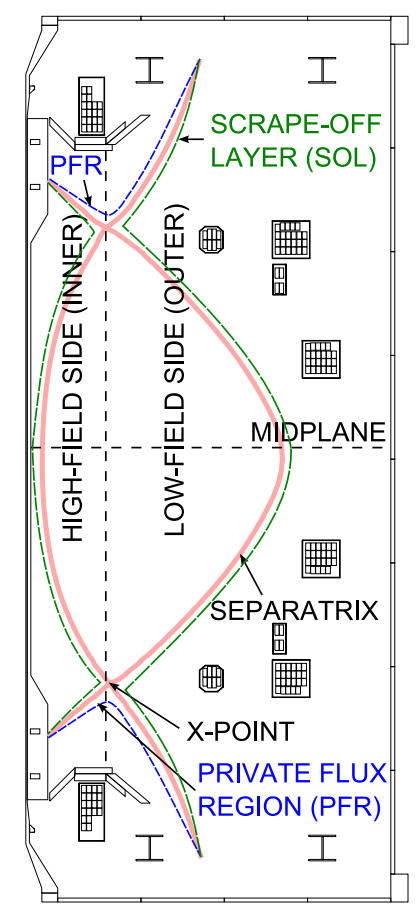

Figure 3.3: An overview of some commonly used terms referring to Tokamak magnetic geometry. The scrape-off layer (SOL) is a region that extends radially from the separatrix to the wall, however, normally only a thin region close to the separatrix is simulated in modelling codes.

interpretation of data from Langmuir probes will first be reviewed.

The most straightforward Langmuir probe configuration is a single conductor immersed in a plasma and connected to a power supply. Normally, such probes are operated by connecting the probe to a sweeping bias voltage, and the current through the probe is recorded. An idealised current-voltage characteristic of a swept probe immersed in a plasma is shown in (3.3.1). Due to the formation of electrostatic sheaths, it not possible for an unbiased probe to access the conditions of the plasma, and so it is important to consider the presence of the sheath when analysing probe data.

If a probe is biased sufficiently negatively, an electrostatic sheath cannot form at the surface of the probe, as all incident electrons are repelled from the probe surface. As a result, only incident ions, which are accelerated by the negative potential, reach the surface of the probe and are collected. In this circumstance, the current reaching the probe is the ion saturation current.

If the probe is left unbiased, the presence of the electrostatic sheath will act to equalise the fluxes of ions and electrons to the probe surface. As a result, the probe will not draw any current from the plasma. The potential of such an unbiased probe is called the floating potential, as the probe is essentially electrically floating at this point. If the random Maxwellian particle flux is given by: 


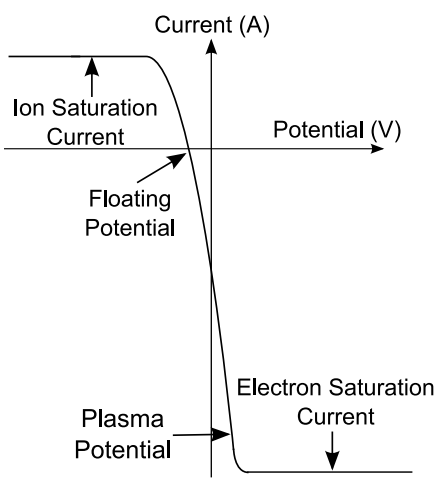

Figure 3.4: Schematic of a typical current-voltage characteristic recorded from a Langmuir probe immersed in a plasma.

$$
\Gamma=\frac{1}{4} n \bar{v}=\frac{1}{4} n \sqrt{\frac{8 k T_{e}}{\pi m_{e}}}
$$

where $n$ is the particle density and $\bar{v}$ is the mean particle velocity, then by employing the Boltzmann relation to electrons incident on an electrically floating probe, the electron flux is given by:

$$
\Gamma_{e}=\frac{1}{4} n_{s e} c_{s} e^{\frac{e \phi_{f}}{k T_{e}}}
$$

where $\phi_{f}$ is the floating potential of the surface, $c_{s}$ is the plasma acoustic velocity and $n_{s e}$ is the electron density at the sheath edge $\approx \frac{1}{2} n_{e}$, roughly half the electron density in the bulk plasma. The ion flux to the probe can be approximated by the one-way Maxwellian flux given in (3.1). Therefore, for a floating probe, $\Gamma_{e}=\Gamma_{i}$, giving:

$$
\frac{e \phi_{f}}{k T_{e}}=\frac{1}{2} \ln \left[\left(\frac{2 \pi m_{e}}{m_{i}}\right)\left(1+\frac{T_{i}}{T_{e}}\right)\right]
$$

If the probe is biased strongly positively, the probe will attract all incident electrons and repel incident ions from its surface. The current drawn from the probe in this configuration is known as the electron saturation current. As shown in (3.3.1), the electron saturation current is often significantly larger than the ion saturation current. This poses difficulties in terms of experimentally determining the electron saturation current, which can damage the probe surface. The total current emitted by a Langmuir probe can be approximated by [41]:

$$
I_{p}=I_{\text {sat }}^{+}\left(1-\frac{e\left(\phi_{\text {bias }}-\phi_{f}\right)}{k T_{e}}\right)
$$

The floating potential $\phi_{f}$ and the ion saturation current $I_{\text {sat }}^{+}$can be determined from 
the current-voltage characteristic recorded at the probe, allowing $T_{e}$ to be ascertained by fitting (3.4) to the data prior to the probe entering electron saturation. The electron density, $\mathrm{n}_{e}$ can then be calculated using the expression for the ion saturation current (assuming the Bohm criterion when $\mathrm{T}_{e}=\mathrm{T}_{i}[8]$ ):

$$
j_{\text {sat }}^{+}=e n_{e} c_{s}
$$

where the relationship between $I_{\text {sat }}^{+}$and $j_{\text {sat }}^{+}$is given by:

$$
j_{\text {sat }}^{+}=\frac{I_{\text {sat }}^{+}}{A_{\text {coll }}}
$$

where $\mathrm{A}_{\text {coll }}$ is known as the collecting area of the probe, given by the projected area of the probe along magnetic field lines. Furthermore, the value of the plasma potential can be approximated by [42]:

$$
\phi_{p}=\phi_{f l}+2.5 \frac{T_{e}}{e}
$$

where $\phi_{p}$ is the plasma potential, $\phi_{f l}$ is the floating potential and $T_{e}$ is the electron temperature $(\mathrm{eV})$. The Langmuir probe system on MAST [43] is operated whereby the probes are multiplexed such that groups of 16 probes are driven by a single power supply. Each probe is swept with a waveform scanning from -200V to 0V (with respect to the floating potential) with a $65 \mu$ s period, so an individual probe is swept every $\approx 1 \mathrm{~ms}$. The spatial resolution of the Langmuir probe diagnostic depends upon how the diagnostic is configured (i.e. which probes are connected to power supplies). It can be taken that in this study, the spatial resolution lies between from $6 \mathrm{~mm}-9 \mathrm{~mm}$ at the lower inner and outer strike points. The data is analysed by determining $j_{\text {sat }}$ from the probe $\mathrm{I}$ $\mathrm{V}$ characteristic, and fitting this characteristic (which does not enter electron saturation region) with (3.4) to obtain $T_{e}, n_{e}$ is calculated using (3.5), assuming $T_{i}=T_{e}$.

\subsubsection{Spectroscopy}

The two principal spectroscopic diagnostics commissioned by the author for this study are based on the Czerzy-Turner (CZ) design and have $1000 \mathrm{~mm}$ and $300 \mathrm{~mm}$ focal lengths. The spectrometers were manufactured by Princeton Instruments, with model numbers AM-510 and SP2300i respectively, with optics designed to image multiple light sources. The AM510 spectrometer is fitted with an 1800 grooves $/ \mathrm{mm}$ grating, giving a linear dispersion of $\sim 0.13 \AA$ and an instrument function $\sim 0.4 \AA$ wide (full-width halfmaximum) with a slit width of $100 \mu \mathrm{m}$. The SP2300i spectrometer was commissioned during this study, and is fitted with a rotatable turret containing three gratings of ruling density 300, 600 and 1200 grooves $/ \mathrm{mm}$. The notable feature of the SP2300i spectrometer is its detector, an electron multiplication CCD (EMCCD), a Princeton 
Instruments ProEM 1024b. This combination of detector and gratings allow spectra to be recorded with ranges from $138-29 \mathrm{~nm}$ with spectral resolution ranging from 0.3 $0.06 \mathrm{~nm}$ (FWHM, $25 \mu \mathrm{m}$ slit width). This sensitive detector, coupled with the optically fast (f/3.7) SP2300i increases the diagnostic signal:noise ratio, permitting measurement of weak spectral lines. The input optics into the spectrometer were designed in order to couple an extended light source to the spectrometer with minimal losses, whilst ensuring that the cross-talk between adjacent input fibres was reduced.

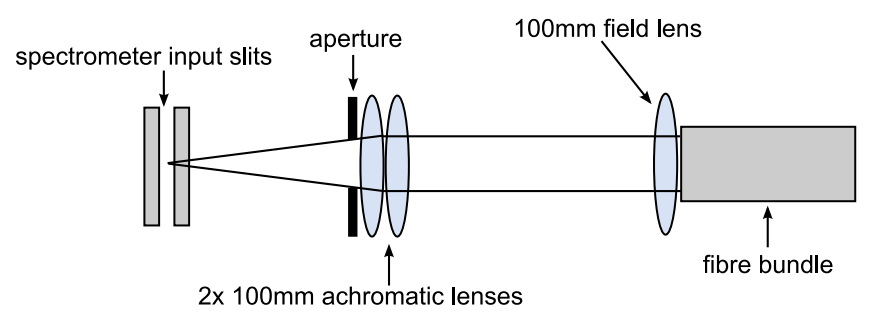

Figure 3.5: Input optics for SP2300i spectrometer

All lenses used in the input optics are assembled from quartz, to ensure good light transmission at near-UV wavelengths. A field lens is positioned close to the fibre bundle to reduce ray divergence from the input fibres, increasing the light reaching the focussing lenses. Two chromatically corrected lenses are used to focus the light onto the spectrometer input slits to ensure good image quality and hence reduce cross-talk between adjacent fibres. An aperture was placed in front of the focussing lenses in order to give a wider input acceptance angle to match that of the input fibres, by reducing the effective lens diameter.

Spectroscopy is an important means of diagnosing low-temperature plasmas, as light emitted from ion-electron recombination events taking place at low temperatures can be used to infer the electron temperature. If the ions and electrons are in coronal equilibrium, the population $N$ of a given state of principal quantum number $n$ is given by $[17]$ :

$$
N \propto \frac{n^{2}}{T_{e}^{\frac{3}{2}}} \exp \left(\frac{13.6}{n^{2} T_{e}}\right)
$$

Therefore, measurements of the intensity of Balmer emission lines can facilitate a measurement of the electron temperature $T_{e}$. Measurements of the chord-integrated electron density $n_{e}$ are made possible by careful analysis of the shapes of Deuterium Balmer emission lines due to the high electron density in detached plasmas.

In high-density, low-temperature plasmas, a spectral line shape can become dominated by Stark broadening [44]. This form of line broadening is brought about by electric microfields generated by Coulomb interactions between an ion and its neighbours. These microfields manifest as a perturbation of the ionic energy states and result in a 
shift in the radiation emitted by electrons residing within the ion's energy states. The gross effect of these perturbations is a spectral line broadening closely resembling a Lorentzian profile which dominates over Gaussian thermal broadening. Passive spectroscopic techniques to characterise plasmas are attractive due to their comparatively low cost (compared with a Thomson scattering diagnostic, for example) and ease of experimental setup. Difficulties arise in data analysis, however, as analysis of Stark broadened lines often involves comparing line widths to tabulated values to calculate the electron density, or fitting experimentally observed line shapes to theoretically calculated profiles, such as in [45]. In this study, the latter approach has been adopted in order to make the data analysis procedure as robust as possible.

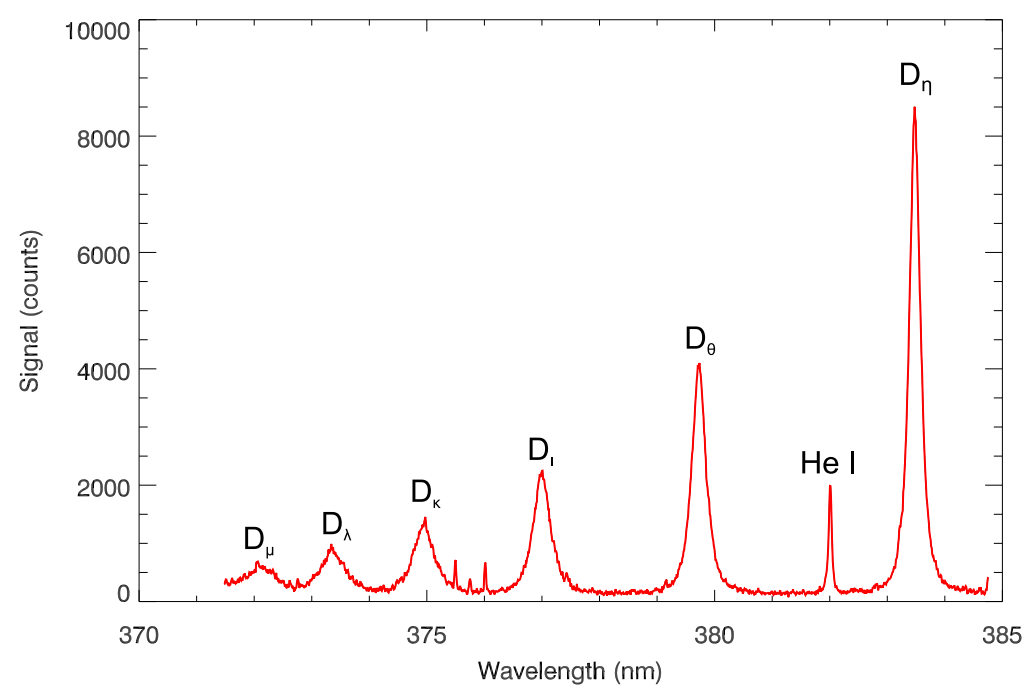

Figure 3.6: Spectrum of high-n Balmer emission lines emanating from the lower inner strike point from shot 23930 at $190 \mathrm{~ms}$.

The onset of detachment on MAST can be detected using filtered photomultiplier tubes (PMTs) were used to measure the brightness of $D_{\alpha}$ and $D_{\gamma}$ spectral lines with a low probability of detector saturation. Light input into the photomultiplier tubes was collected using a vessel-facing lens to focus light onto an optical fibre. The fibre was input into a beam-splitter, which served as input into two PMTs, filtered for each of the spectral lines. Due to the relatively poor quantum efficiency ( $\sim 10 \%)$ of PMTs, detector saturation is more easily avoided compared with more sensitive detectors, such as CCDs. Furthermore, the temporal resolution of the PMT-based line ratio measurement is $65 \mu \mathrm{s}$, facilitated by signal amplification within the detector. The temporal resolution of the PMTs used is limited by the analogue-to-digital converter, and is very high compared with several milliseconds for CCD-based measurements. 


\subsubsection{Spectroscopic $\mathrm{T}_{\mathrm{e}}$ Determination}

In recombining plasmas, the line averaged (along a spectroscopic line of sight) electron temperature can be measured by measuring the intensities of spectral line in the recombination spectrum. The absolute intensity of a spectral line (measured in $\mathrm{m}^{-3} \mathrm{~s}^{-1}$ ) brought about by an electronic transition from a state with principal quantum number $\mathrm{j}$ to one of state $\mathrm{i}$ is given by [46]:

$$
I_{i j}=n_{j} A_{i j}
$$

where $\mathrm{j}>\mathrm{i}, A_{i j}$ is the spontaneous transition probability from state $\mathrm{p}$ to $\mathrm{k}$, and $n_{j}$ is the population density of state $\mathrm{j}$. Consider an atom in which the populations of states with principal quantum number $\mathrm{i}$ and $\mathrm{j}$ are due to three processes [47]:

1. Spontaneous recombination

This is the recombination of an ion with a free electron of momentum $p$.

$$
\mathrm{H}^{+}+\mathrm{e}^{-} \rightarrow \mathrm{H}+h \nu
$$

The rate of this process can be given by:

$$
R_{r e c, s}(p)=n_{j+1} n_{e}(p) A
$$

where $n_{j+1}$ is the density of singly ionised atoms (where state $\mathrm{j}$ corresponds to neutral atoms), $n_{e}(p)$ is the density of free electrons of momentum $p$ per unit momentum interval, given by the Maxwell-Boltzmann distribution and $A$ is the probability per unit time of this event occurring.

2. Induced recombination

This is a similar process to spontaneous recombination, except it is initiated by a photon of energy $h \nu$, its rate is given by:

$$
R_{r e c, i}(p)=n_{j+1} n_{e}(p) f(\nu) B_{j i}
$$

where $f(\nu)$ is the radiation density at frequency $\nu$, given by the Planck distribution and $B_{j, i}$ is the probability per unit time per unit spectral radiance of this occurring.

3. Photoionization

In this processes, a photon is absorbed by a bound electron, causing the formation of an ion 


$$
\mathrm{H}+h \nu \rightarrow \mathrm{H}^{+}+\mathrm{e}^{-}
$$

The rate at which this occurs can be given by:

$$
R_{p i}=n_{j} f(\nu) B_{i j}
$$

where $B_{i j}$ is the probability per unit time per unit spectral radiance of this event occurring.

In thermal equilibrium, the rate of recombination and ionization must equalise to ensure detailed balance. This can be expressed as:

$$
R_{r e c, s}(p)+R_{r e c, i}(p)=R_{p i}
$$

or

$$
n_{j} n_{e}(p) A_{j i}+n_{j} n_{e}(p) f(\nu) B_{j i}=n_{i} f(\nu) B_{i j}
$$

Taking the population densities of states $i$ and $j$ to follow the Boltzmann distribution [47]:

$$
\frac{n_{i}}{n_{j}}=\frac{g_{i}}{g_{j}} \exp \left(\frac{-h \nu}{k_{B} T}\right)
$$

where $g_{i}$ and $g_{j}$ are the statistical weights of states of states $i$ and $j$ respectively, it can be shown that [48]:

$$
\frac{B_{i j}}{B_{j i}}=\frac{g_{i j}}{g_{j i}} g_{e}\left(\frac{4 \pi p^{2}}{h^{3}}\right)
$$

and

$$
\frac{A_{j i}}{B_{j i}}=\frac{8 \pi h \nu^{3}}{c^{3}}
$$

and using the following statements of the Maxwell-Boltzmann and Planck distributions:

$$
\begin{gathered}
n_{e}(p)=\frac{n_{e} 4 \pi p^{2}}{\left(2 \pi m k_{B} T\right)^{\frac{3}{2}}} \exp \left(\frac{-p^{2}}{2 m k_{B} T}\right) d p \\
f(\nu)=\frac{2 h \nu^{3}}{c^{2}} \frac{1}{e^{\frac{h \nu}{k_{B} T}}-1}
\end{gathered}
$$


then $(3.16)$ can be re-cast as [49]:

$$
\frac{n_{j} n_{e}}{n_{i}}=\frac{\left(2 \pi m k_{B} T_{e}\right)^{\frac{3}{2}}}{h^{3}} \frac{2 g_{j}}{g_{i}} \exp \left(\frac{h \nu}{k_{B} T_{e}}\right)
$$

where $g_{e}=2$ (allowing for two electron spin states) was used. Taking the logarithm of (3.22) yields a straight line:

$$
\log \left(\frac{n_{j}}{g_{j}}\right)=\left(\frac{1}{T_{e}}\right) \frac{13.6}{j^{2}}+\log \left(\frac{n_{i} h^{3} n_{e}}{g_{i}\left(2 \pi m k_{B} T_{e}\right)^{\frac{3}{2}}}\right)
$$

where $h \nu=\frac{13.6}{j^{2}}$ has been used, as the ionisation energy of state $j$ in the case of energy level transitions in hydrogen isotopes. Therefore, determination of $n_{j}$ using (3.9) and measurements of $\mathrm{I}_{i j}$ together with (3.23) allow $T_{e}$ to be determined.

Assuming that the errors in the atomic data are small compared with those arising from errors in the experimental data, the dominant source of uncertainty in the evaluation of $\mathrm{T}_{e}$ is in the measurement of $n_{j}$. As $n_{j}$ is computed from the integrated line emission, it is important to consider the degree to which noise effects the determination of the upper state population density. The total noise in the measurement of the shape of a spectral line with a CCD sensor arise due to several contributions. So-called dark current arises in CCDs due to electrons generated within the sensor due to thermionic emission as it warms up during use. The Princeton ProEM camera used in collecting data for $\mathrm{T}_{e}$ determination included Peltier cooling to reduce dark noise. Dark noise was removed during data analysis by subtracting frames where the camera was not exposed to light from the data. Photon (or shot) noise is generated by the random nature of light detection. As the detection of an individual photon is given by the Poisson probability distribution function [50], the number of photons detected will differ from one exposure to another in the presence of a constant intensity light source with all other sources of noise eliminated. The magnitude (standard deviation) of photon noise is given by the square root of the number of photons collected.

Other sources of noise tend to be due to the electronics within the camera system. Analog-to-digital conversion of the electrons generated by the CCD is one such source, which increases if signal amplification is required to boost the signal generated by weak light sources. Other sources of electrical noise associated with camera control, readout, and power supply can manifest as additional sources of noise. Provided that the experimental setup remains fixed whilst measurements are taken, these sources of noise can be quantified and assumed to be invariant during an experiment. In the experimental setup used in this study, the readout noise from the CCD camera is taken to be 10.1 counts, determined using the manufacturer's calibration when the camera was (recently) constructed. Combining the shot noise due to $\mathrm{N}$ photons, $\sigma_{p}$ and electrical 
noise inherent in the camera $\sigma_{e}$ yields:

$$
\sigma_{n}=\sqrt{\sigma_{p}^{2}+\sigma_{e}^{2}}=\sqrt{f N+\sigma_{e}^{2}}
$$

where $f$ is the number of counts per photon, measured by carrying out an absolute intensity calibration, using a source of known spectral radiance. Using this information, the error in $\mathrm{T}_{e}$ is calculated when $\sigma_{n}$ is added to and subtracted from the data. The uncertainty in $\mathrm{T}_{e}$ is then taken to be average deviation of these calculated electron temperatures from $\mathrm{T}_{e}$ calculated from the raw data, as shown in figure 5.14.

In order for this analysis to be valid, the high-n Balmer states must be in thermal equilibrium with background electrons. The lowest-n line for which this method is valid was calculated by Griem [51], by assuming that equilibriation takes place if the collisional depopulation rate of the state is ten times the radiative decay rate. This leads to the expression:

$$
n_{c r}=\left[\frac{10}{2 \sqrt{\pi}} \frac{z^{7}}{n_{e}}\left(\frac{\alpha}{a_{0}}\right)^{3}\right]^{\frac{2}{17}}\left(\frac{T_{e}}{z^{2} E_{H}}\right)^{\frac{1}{17}}
$$

where $\mathrm{z}$ is the ion charge (taken to be 1$), \alpha$ is the fine structure constant $(=1 / 137)$, $a_{0}$ is the Bohr radius $(\approx 0.53 \AA), \mathrm{E}_{H}$ is the Rydberg energy $(13.6 \mathrm{eV})$ and all other terms have their usual meanings. Equation (3.25) calculated for various plasma parameters yields figure 3.7. For plasma conditions $\mathrm{T}_{e} \approx 1 \mathrm{eV}, \mathrm{n}_{e} \approx 10^{20} \mathrm{~m}^{-3}$, intensities of $\mathrm{D}_{\beta}$ $(n=4)$ and higher-n lines are good candidates to use in this analysis. It is preferable to use lower-n lines in order to achieve good photon statistics, but not low enough to invalidate the analysis method outlined.

\subsubsection{Spectroscopic Line Integrated $\mathrm{n}_{\mathrm{e}}$ Determination}

In high density plasmas, the emission of photons can be interrupted by the presence of nearby free ions and electrons due to collisions and the generation of electric fields. As part of this study, the effects of Stark broadening on the widths of high-n Balmer emission lines was investigated using results from a Model Microfield Method (MMM) code [45]. In this model, the perturbations of free ions and electrons on the emitting ion are treated separately, generating their own plasma microfields. The evolution of the microfields is modelled as a stochastic process, with fluctuation times being the reciprocal of the ion and electron plasma frequencies. As a result, the electron microfield evolves significantly more quickly (by a factor of $\approx 60$ ), the average electron microfield is evolved to allow the code to evolve on the longer ion timescale. At each time step, the microfields are assumed to take a constant value, calculated using ion and electron field distribution functions. Unlike calculations carried out by Griem [52], 


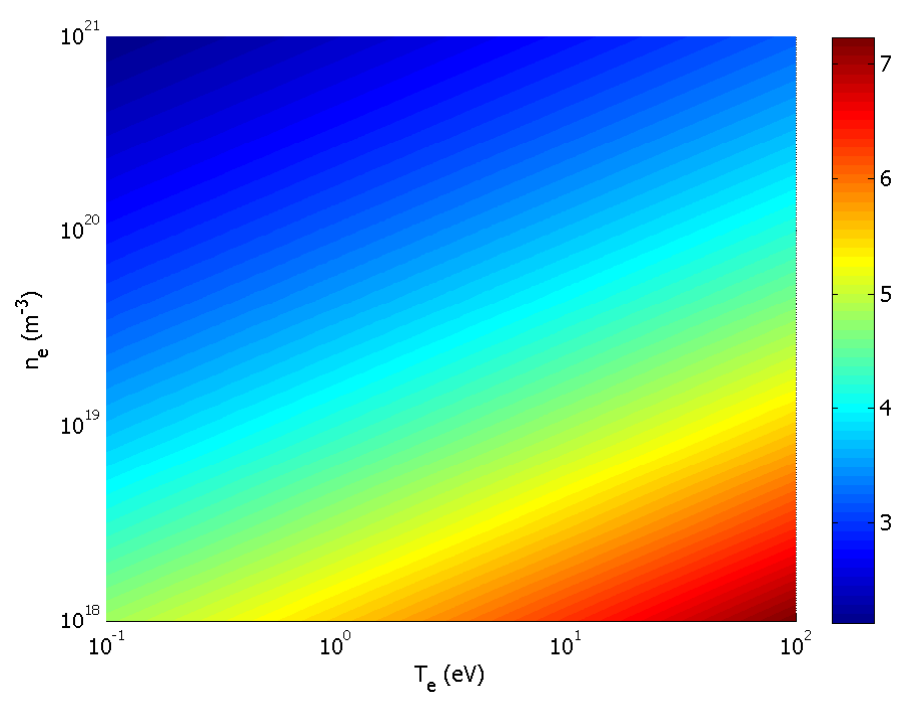

Figure 3.7: Calculation of the lowest-n state that can be taken to be in partial local thermodynamic equilibrium with free electrons.

the effects of moving perturbing ions is included in the calculation.

The plasma parameters over which this analysis can be applied is limited by the Inglis-Teller limit [53] (figure 3.8), which quantifies the highest resolvable upper principal quantum number line $n_{\max }$ before they start to merge to form a continuum. As Stark broadening is more pronounced at higher density, line broadening increases as the Inglis-Teller limit decreases. A concise form of this limit is:

$$
\log n_{e}=29.26-7.5 \log n_{\max }
$$

A further constraint is that the mean interelectronic distance is smaller than the electron Deybe length. The mean interelectronic distance, $r_{0}(\mathrm{~m})$, is defined as:

$$
\frac{4}{15}(2 \pi)^{\frac{3}{2}} r_{0}^{3} n_{e}=1
$$

which limits the range of applicable electron densities and temperatures to [45]:

$$
\frac{r_{0}}{\lambda_{d}}=\left(8.34 \times 10^{-5}\right) \frac{n_{e}^{\frac{1}{6}}}{T_{e}^{\frac{1}{2}}}<1
$$

For all plasma conditions of interest in this study, this criterion is satisfied.

In high density divertor plasmas, such as those observed in Alcator C-Mod, the Inglis-Teller limit is approximately 11 for $n_{e} \approx 1.8 \times 10^{21} \mathrm{~m}^{-3}$ [17]. If spectroscopic data is available for the region of the spectrum where the emission lines form a continuum, it is possible to estimate the electron density by using the Inglis-Teller limit and knowledge 


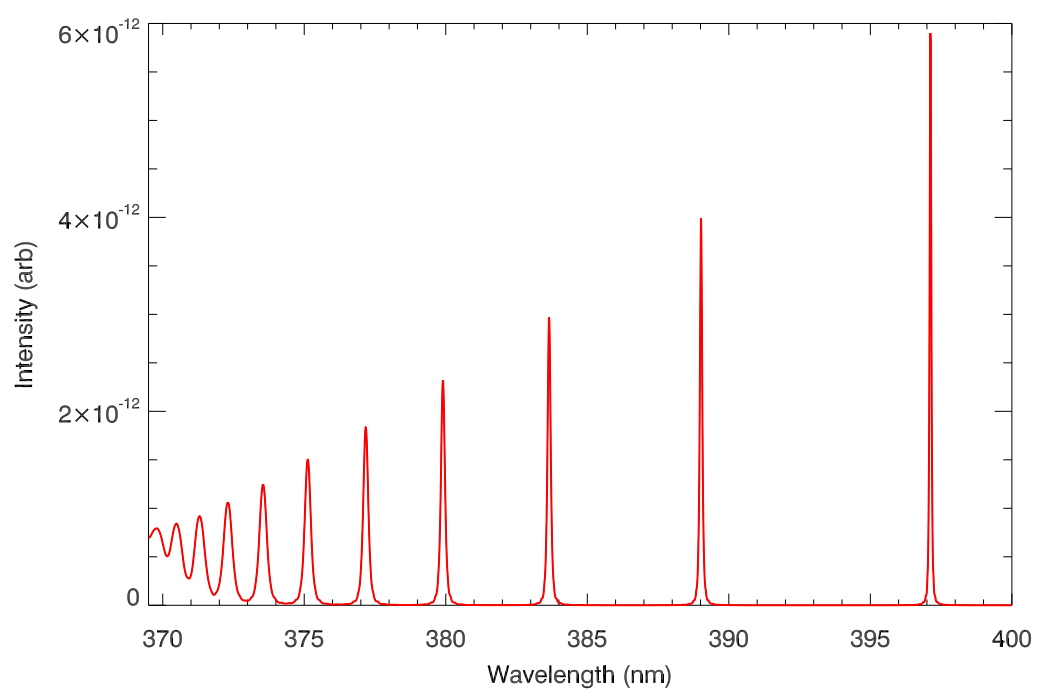

Figure 3.8: Calculated Stark broadened spectrum of Deuterium high-n Balmer lines, $n_{e}=5.0 \times 10^{19} \mathrm{~m}^{-3}, T_{e}=1 \mathrm{eV}$.

of the position of the shortest wavelength series emission line before a continuum is formed. However, at lower density divertor conditions, such as $n_{e} \approx 1 \times 10^{20} \mathrm{~m}^{-3}$, $n_{\max } \approx 17$. The low brightness of such high-n lines can prohibit measurements of the continuum in lower density conditions without making sacrifices in terms of temporal resolution, as higher exposure times become necessary to acquire data.

In this study, line-integrated $n_{e}$ measurements were made by analysing the shape of the $\mathrm{D}_{\epsilon}(397 \mathrm{~nm})$ line and comparing them to theoretically calculated line shapes calculated by a code documented in [45]. An example Stark broadened spectrum calculated by the code is shown in figure 3.8. In order to account for (albeit small) Doppler broadening of spectral lines, $T_{e}=1 \mathrm{eV}$ is used, and calculated Doppler broadened line shapes are convolved with the calculated Stark broadened line shape. Account must also be taken of the instrumental broadening of the spectrometer making the line shape measurement. The measured instrument function for different slit widths is recorded in the region where the instrument function can be well approximated by a Gaussian profile. In order to do this, the measured line shapes are then fitted to Gaussian profiles, and the line width (FWHM) is recorded for a number of slit widths. It is then possible to calculate the instrument function for reasonable $(\leq 100 \mathrm{~nm}$ in this case) slit widths. For a given slit width and calculated Stark+Doppler broadened line shape, the theoretical profile is convolved with the calculated instrument function and fitted to a given spectral line in a least-squares manner.

Experimental uncertainties in the fitting procedure take into account the accuracy of the line shape calculation $(\approx 10 \%[45])$ and a the sensitivity of the line shape to 
deviations from the density calculated from the fitting procedure. It was found that with the more accurate measurement of the spectral line shape afforded by the AM510 spectrometer results in a reduced degree of uncertainty in the calculated line integrated $\operatorname{density}(\approx 15 \%)$. Conversely, the less accurate line shape measurements taken with the SP2300i instrument result in $10 \%$ greater uncertainty in the line integrated density calculated by the fitting procedure.
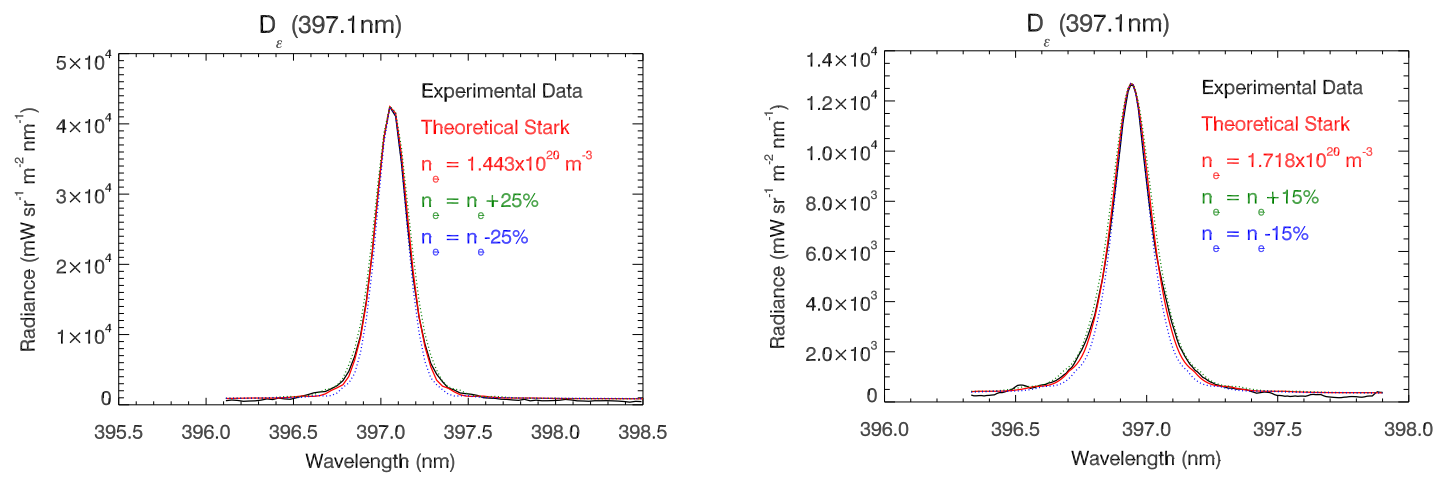

Figure 3.9: The uncertainty in least-squares fitting of the Balmer line shape is calculated by considering the sensitivity of the calculated line shape to the input line integrated density. Left: results of a Stark broadening analysis carried out on SP2300i data, right: Stark broadening analysis of AM510 spectrometer data.

The magnetic field of MAST will also have an effect on the shape of recorded spectra via Zeeman splitting of spectral lines. Zeeman splitting occurs when electronic transitions occur in the presence of a magnetic field that effectively splits atomic energy levels [47]. According to Griem [51], the magnitude of Zeeman splittings are of the order of the electron cyclotron frequency, which allows the relative wavelength shift to be calculated as:

$$
\begin{gathered}
\frac{\omega_{c e}}{\omega} \approx \frac{\Delta \lambda}{\lambda} \\
\Delta \lambda \approx\left(\frac{e B}{m_{e}}\right)\left(\frac{\lambda}{2 \pi c}\right) \lambda
\end{gathered}
$$

For the MAST magnetic field (taken to be 1T) the wavelength shift over the Deuterium Balmer series would range from $0.04 \mathrm{~nm}$ for $\mathrm{D}_{\alpha}(656 \mathrm{~nm})$ and $0.01 \mathrm{~nm}$ at the continuum (taken to be $365 \mathrm{~nm}$ ). Therefore, as the properties of high-n Balmer lines are of interest in this study, the effects of Zeeman splitting on the measured spectra can be taken as negligible. This estimate is in qualitative agreement with another method of calculating the effect of Zeeman splitting, by taking the intervals of the split lines to be [52] in the Paschen-Back limit (valid for strong fields of the order of 10's of Tesla): 


$$
\Delta \omega_{z}=\left(n_{i}-1\right)\left(\frac{e B}{2 m c}\right)
$$

where $\mathrm{n}_{i}$ is the initial quantum number of the electronic transition producing the line. Comparing this to estimated Stark broadening widths, it can be shown that for Balmer lines, line broadening due to the Stark and Zeeman broadening effects become comparable at magnetic fields exceeding $1 \mathrm{~T}$.

The effects of neutral perturbers gives rise to resonance and Van der Waals broadening, the effects of which are not accounted for in the theoretical calculations of Stark broadened line shapes. Resonance broadening is due to collisions with neutral atoms of the same species, in this case, neutral Deuterium atoms, however, in order for resonance-broadening to have a significant effect (of the order of tenths of Angstroms) the density of neutral perturbers must be of the order of $10^{24} \mathrm{~m}^{-3}$ in the case of $\mathrm{D}_{\gamma}$ emission [54]. Therefore, the effects of resonance broadening on high-n Balmer emission lines in Tokamak conditions are significantly below the resolution of the diagnostics used in this study. Van der Waals broadening arises due to the interaction of the emitting ion with Van Der Waals forces due to neighbouring atoms. However, like resonance broadening, the effects of Van der Waals broadening in Tokamak conditions is a minor contribution to the overall line shape [55].

Finally, natural line broadening is due to the small spread in the energy $\Delta E$ of quantum states due to the Heisenberg uncertainty principle [48]:

$$
\Delta E \Delta t \approx \bar{h}
$$

where $\Delta t$ is given by the lifetime of an atom in an excited state. Therefore, using the wavelength broadening $\Delta \lambda$ of a spectral line due to this effect is:

$$
\Delta \lambda \approx \frac{\lambda^{2}}{2 \pi}\left(\frac{1}{\Delta t_{i}}-\frac{1}{\Delta t_{f}}\right)
$$

where $\Delta t_{i}$ and $\Delta t_{f}$ are the lifetimes of the initial and final states respectively. Given that for Balmer series transitions, $\Delta t_{i, f}$ are of the order of $10^{-8} \mathrm{~s}$, the natural line width is very small, approximately $10^{-4} \AA$.

\subsubsection{Thomson Scattering}

One of the principal diagnostics on MAST is the Thomson Scattering system [56]. The diagnostic is based on the scattering of light emitted from $830 \mathrm{~Hz} 1.6$ Joule lasers directed into the plasma. The light is collected with a collection lens and directed into optical fibres, where it is analysed with polychromators. From analysis of the scattered light, electron density and temperature profiles can be obtained with high spatial and 
temporal resolution.

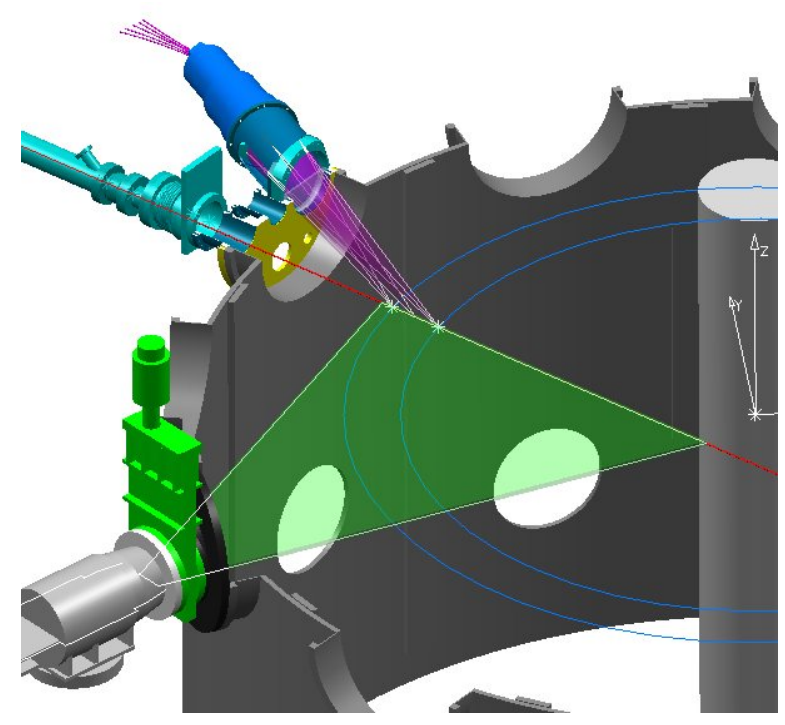

Figure 3.10: Laser path (red) collection optics of the MAST core (green) and edge (purple) Thomson scattering systems.

The MAST Thomson Scattering system offers the flexibility to provide temperature and density profiles with a temporal resolution of approximately $4.2 \mathrm{~ms}$ if the lasers are fired evenly, or can be fired quick succession to measure 8 plasma profiles in bursts with a $5 \mu$ s spacing. Data from 120 spatial locations are recorded from radial locations ranging from $0.24-1.5 \mathrm{~m}$ with $10 \mathrm{~mm}$ resolution. Some notable applications of Thomson Scattering on MAST has been in the diagnosis of filaments [57], pedestal evolution [58], the structure of magnetic islands and sawteeth.

Thomson Scattering data is used in this study as a measurement of upstream electron density and temperature profiles in the edge of MAST plasmas, to facilitate modelling efforts. This information is extremely important in order to ascertain the role and magnitude of gradients in plasma properties in the plasma boundary, and as a strong constraint on interpretive modelling codes.

\subsubsection{Infrared Cameras}

Infrared (IR) cameras have been deployed in a wide variety of scenarios with the purpose of ascertaining the surface temperature of an object with high temporal resolution. On Tokamaks, IR cameras have become a standard diagnostic to measure the temperature of, and heat flux to plasma-facing components. Such measurements are increasingly important in order to derive experimental scalings for the heat flux to plasma-facing components in order to estimate their magnitude in next-step devices such as ITER. As noted in a previous section, Langmuir probes offer the possibility of estimating the 

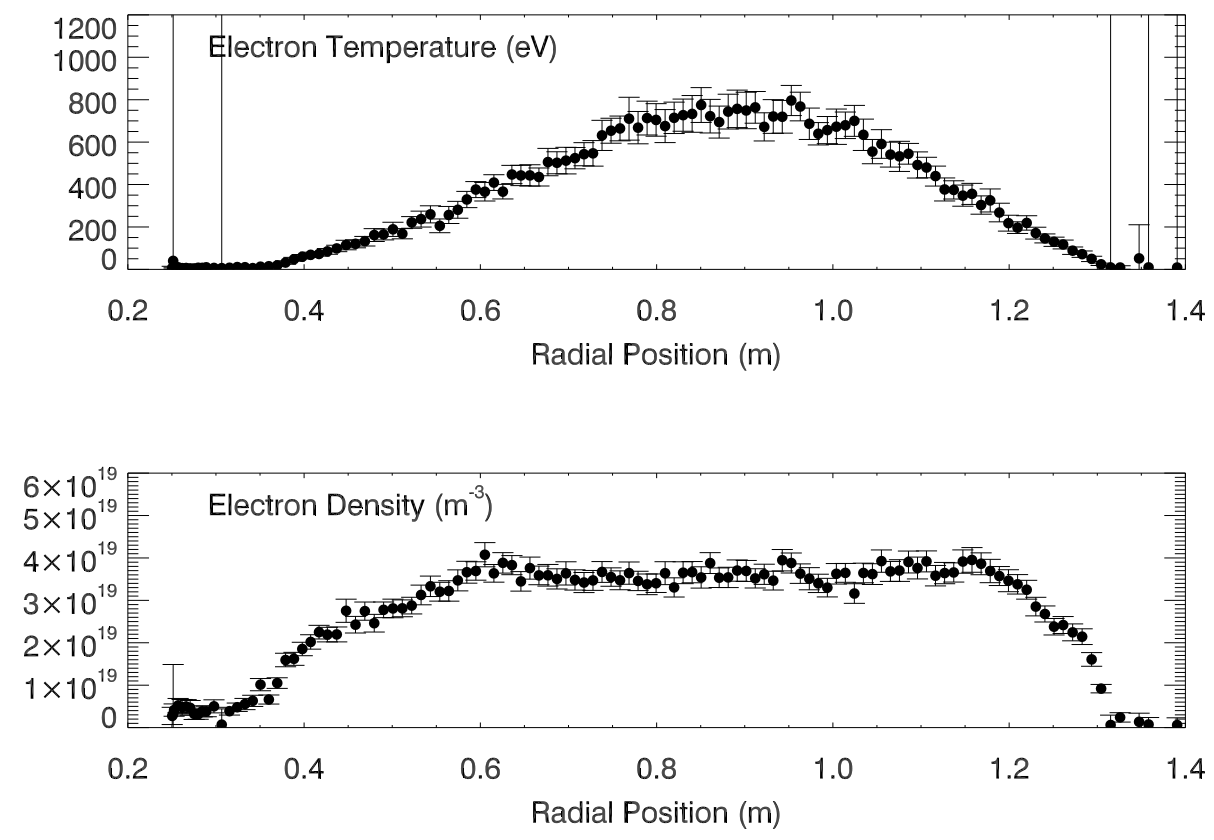

Figure 3.11: Data taken from the MAST Thomson scattering diagnostic on shot 24703 at $212 \mathrm{~ms}$.

net power to the surface of the probe, which if embedded in a divertor tile, is strongly related to the power incident on the surface of the divertor. However, this power is estimated only with knowledge of the electron contribution to the incident power, and so approximations are needed to estimate the ion contribution. IR cameras, conversely, can be used to infer the net heat flux to a plasma-facing surface, irrespective of the plasma species transporting the power. However, interpretation of surface temperatures and heat fluxes to surfaces by IR thermography is complicated by the effects of surface layers with poor thermal contact with the bulk material and the presence of dust. Recent investigations into IR analysis using long and medium wavelength cameras [40], have since improved the reliability of these measurements on MAST.

In detached divertor conditions, broad-band bremsstrahlung emission from electromagnetic interactions between plasma ions and electrons can result in contamination of measurements of surface temperatures and heat fluxes using infra-red cameras. To ascertain if such contamination is likely in MAST plasmas, the blackbody photon flux was compared a line-integrated bremsstrahlung emission through $1 \mathrm{~m}$ of homogeneous plasma (figure 3.12). The bremsstrahlung emission spectrum was integrated between $4.5 \mu \mathrm{m} \rightarrow 5 \mu \mathrm{m}$, corresponding to the bandpass of the filter used in MASTs medium wavelength IR camera. This calculation has a number of associated uncertainties, such as the surface blackbody emissivity (assumed here to be unity), and plasma 


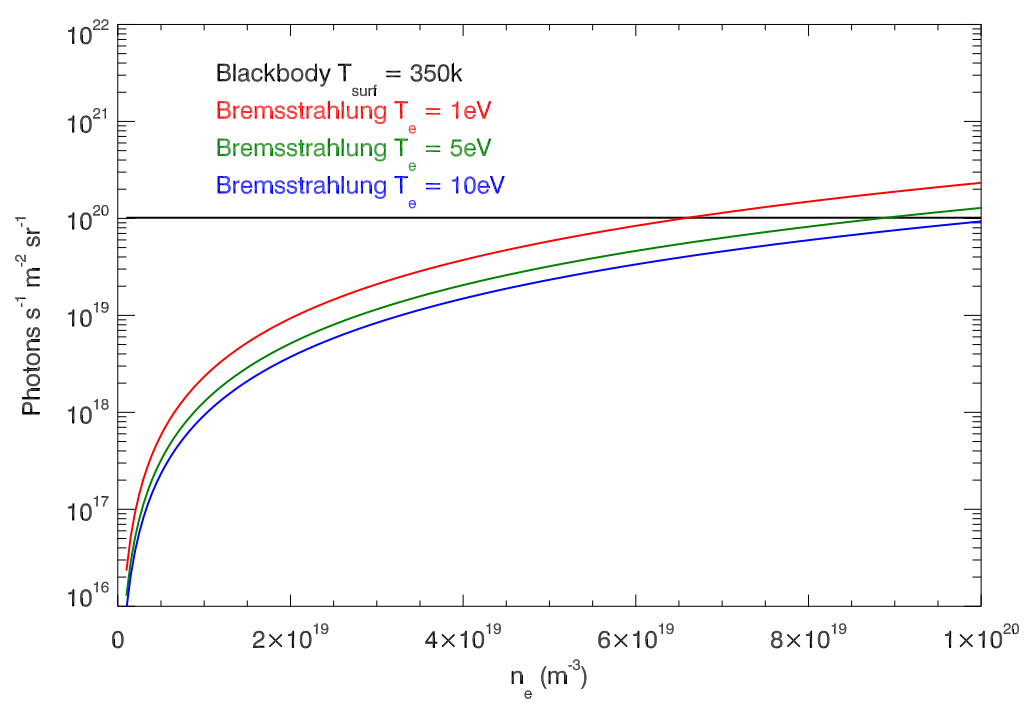

Figure 3.12: Simulated blackbody photon flux from a 350K perfect blackbody compared with a line integrated (path length $=1 \mathrm{~m}$ ) bremsstrahlung photon flux through a homogeneous plasma, varying electron temperature and density.

conditions in a Tokamak divertor leg are not necessarily uniform. Furthermore, the effects of electron-neutral bremsstrahlung emission [59], which could be significant at high neutral pressures. However, the calculation does suggest that, under detachmentlike conditions, bremsstrahlung emission could complicate IR measurements of surface temperatures, as observed on other devices [18].

\subsubsection{Visible Imaging}

MAST is equipped with several visible imaging diagnostics. Their purpose varies from general plasma and machine monitoring to narrow-band imaging spectroscopy. This study concentrates solely on the latter purpose, as imaging spectroscopy is an important means of characterising plasma behaviour, in particular in divertor detachment, where diagnosics such as Langmuir probes are less effective.

The narrow-band imaging diagnostic used in the MAST divertor is DivCam. Light enters DivCam through an aperture that is positioned in front of a MAST window. From here, the light passes through telecentric optics to make the principal rays parallel, before passing into a beam-splitter to divide the input light. This split light then passes through a field lens, focussing the light that passes through a filter wheel containing narrow-band optical filters before reaching two CCD detectors.

The CCD detectors are monochrome $1024 \times 1024$ chips with $7.4 \mu \mathrm{m}$ square pixels with an interline-transfer architecture to avoid smearing during readout. Operating in fullframe mode, the camera is capable of $48 \mathrm{~Hz}$ frame rate. Using $2 \times 2$ binning, however, the 


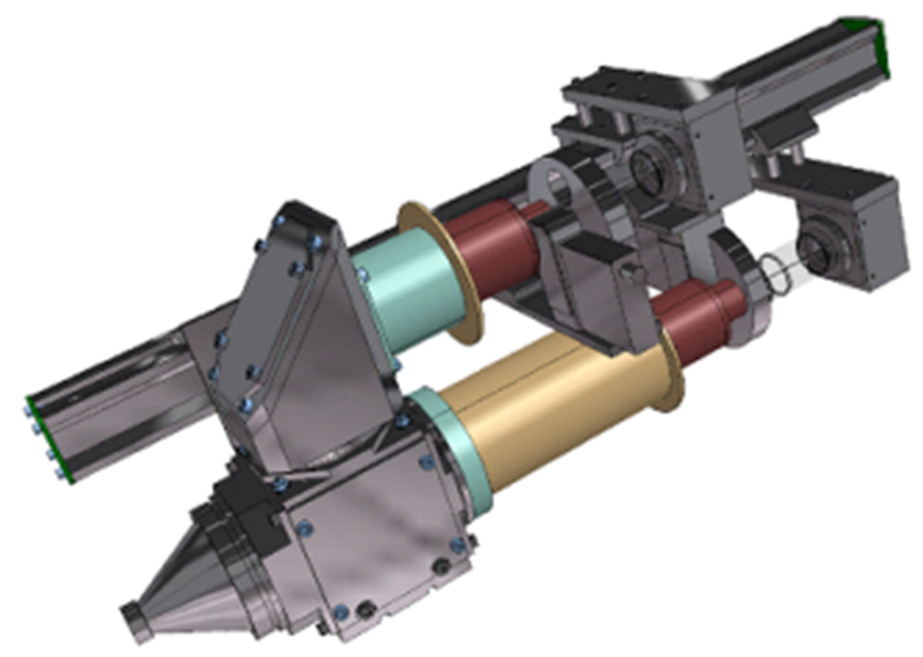

Figure 3.13: CAD model of DivCam.

frame rate can be doubled to $96 \mathrm{~Hz}$, at the loss of spatial resolution in the horizontal and vertical directions. The principal applications of DivCam are for characterising the MAST boundary plasma using Deuteriun Balmer $\left(\mathrm{D}_{\alpha}, \mathrm{D}_{\beta}, \mathrm{D}_{\gamma}\right.$ and $\left.\mathrm{D}_{\delta}\right)$ and He I emission lines [60] and the monitoring of divertor strike-point characteristics.

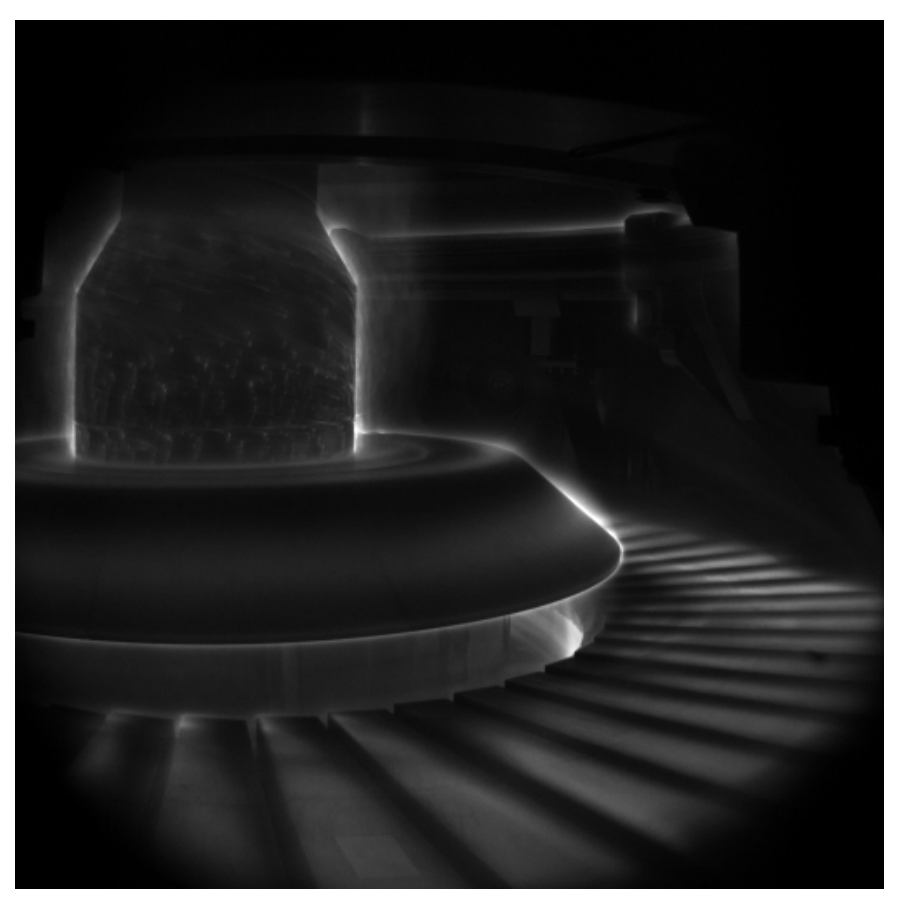

Figure 3.14: A MAST disruption in $\mathrm{D}_{\beta}$ radiation as seen from the lower divertor. 
To capture events with greater temporal resolution, two high-speed Photron APXRS cameras can be used to replace two standard DivCam cameras for high-speed imaging. Such capability is important when studying strongly time-dependent behaviour, such as edge-localiased modes (ELMs), sawteeth, or the evolution of detachment. Photron cameras are not used routinely in conjunction with DivCam due to the inability to couple two Photron APX-RS cameras to a DivCam unit, and, more importantly, the decreased dynamic range of the APX-RS camera (maximum 10-bit) compared to the Imperx cameras normally employed on DivCam (maximum 12-bit).

Quantitative analysis of DivCam data requires absolute calibration of the lens, filter and camera configuration with a suitable light source and a Lambertian surface in order to convert the signal levels recorded by the cameras for a given exposure time into a photon flux across the field of view. This cannot be taken as uniform across the field of view as vignetting strongly deteriorates signal levels at the edges of the detector.

Good characterisation of distortions brought about by the imaging optics is also essential in order to ensure a good mapping between pixel locations on a camera and locations seen on the MAST vessel. This mapping is calculated using the LEON code, written for mapping the positions of the different digital cameras used on MAST. Armed with this information, it is possible to reconstruct poloidal emission profiles of specific lines as seen by the camera. The calculation of poloidal emission profiles from filtered camera data amounts to solving the linear problem:

$$
A x=b
$$

where $\boldsymbol{x}$ is the poloidal emission profile, $\boldsymbol{b}$ is the measured brightness of each viewing chord (pixel), and $\boldsymbol{A}$ is a matrix describing the relationship between $\boldsymbol{x}$ and $\boldsymbol{b}$. In this problem, the poloidal emission profile is over-sampled by the diagnostic, as the same poloidal location is imaged by the camera at multiple locations. As a result, popular algorithms for solving the above overdetermined linear problem are SVD (singularvalue decomposition), conjugate-gradient or maximum entropy. Problems can arise when using SVD and conjugate-gradient based algorithms in enforcing a non-negativity constraint, ensuring that the poloidal plasma emissivity is always greater than or equal to zero. Maximum entropy-based algorithms inherently ensure that the solution is non-negative, making it an attractive means of solving (3.34) with physically realistic results [61].

\subsection{Summary}

The extensive diagnostic dataset on MAST including divertor Langmuir probes, IR cameras, Thomson scattering and DivCam has been extended to include line-of-sight 
spectroscopy using a spectrometer developed during the course of this study. Data analysis codes to measure line averaged electron temperatures and line integrated electron densities have been developed using characteristics of recombining deuterium emission spectra that are normally observed during detached divertor conditions. The implications of detachment on the analysis of other diagnostics, such as IR thermography, have been investigated. 


\section{Chapter 4}

\section{OSM Physics Additions}

In this chapter, numerical enhancements made to the OSM code in this study (Appendix A) have facilitated the use of a more sophisticated set of conservation equations that capture additional physical mechanisms. These include thermoelectric and PfirschSchlüter currents, the effects of magnetic flux expansion, accurate calculation of the plasma potential and cross-field drift motion. The intention of this study is to address a loss of parallel pressure balance between the outboard midplane and lower outer divertor target, which is frequently observed in MAST discharges.

\subsection{The Original Model}

The onion skin method, described in section 2.7, requires that the solution of the fluid transport equations along nested flux tubes corresponds to experimentally determined quantities at specific locations. In the original OSM model used in this study, equations of particle (ion) and (total) momentum conservation are used:

$$
\begin{gathered}
\frac{\partial \Gamma_{i}}{\partial s}=S_{p} \\
\frac{\partial}{\partial s}\left(n_{e} T_{e}+n_{i} T_{i}+m_{i} \frac{\Gamma_{i}^{2}}{n_{i}}\right)=S_{m}
\end{gathered}
$$

where $s$ is the distance along a given field line, where $s=0 \mathrm{~m}$ and $s$ is a maximum where flux tubes intersect the surfaces of Tokamak divertor tiles, $\Gamma_{i}=n_{i} v_{\|, i}, n_{e}=n_{i}$ and $T_{i}=\gamma T_{e}$, where $\gamma$ is a user-specified quantity, that can vary spatially. $S_{p}$ is a particle source $\left(\mathrm{m}^{-3} \mathrm{~s}^{-1}\right), S_{m}$ is a momentum source $\left(\mathrm{kg} \mathrm{m}^{-2} \mathrm{~s}^{-2}\right)$. Electron temperature profiles along flux tubes are calculated by fitting measured values of $T_{e}$ at given locations to a model that assumes that heat is transported via electron conduction. Consequently, allowing $T_{e}$ to take prescribed values at specific locations is ensured during this fitting process. 
Ensuring that the solutions to (4.1) and (4.2) coincide with numerous experimental data points at arbitrary locations is handled using particle and momentum sources known as fitting terms. These sources are not ascribed to a given physical mechanism, and are calculated to ensure agreement between the calculated density and ion velocity profiles with experimental measurements irrespective of the conservation laws or numerical scheme used. For example, in order to ensure that the ion flux incident on two surfaces bounding a field line calculated by OSM coincided with measured values, the ion flux at one end of the flux tube $\left(\Gamma_{0}\right.$ when $\left.\mathrm{s}=0\right)$ can be prescribed by an integration constant when (4.1) is integrated along the flux tube. In order to ensure consistency with experimental data at the other end of the integration domain $\left(\Gamma_{t}\right.$ when $\left.\mathrm{s}=\max \right)$, the particle source $S_{p}$ would need to satisfy the following requirement:

$$
\Gamma_{t}=\int_{s=0}^{s=\max } S_{p} \cdot d s+\Gamma_{0}
$$

The particle fitting source ensures that an analogous relationship for all experimental data input into the code is upheld. A momentum fitting source also exists to ensure that solutions for $n_{e}, n_{i}$ coincide with experimental observations. Additional particle and momentum sources can be calculated by the EIRENE kinetic neutral transport code [2] to provide accurate 2D source distributions arising from ionisation of neutrals within the plasma and volume recombination processes. Furthermore, EIRENE can also used to calculate momentum sinks arising from ion-neutral friction During OSM-EIRENE code runs, OSM and EIRENE are iterated to ensure convergence between the two codes and hence to ensure appropriate fitting sources have been calculated.

In the MAST implementation of OSM, information concerning the magnetic flux equilibrium is provided by the EFIT code [62], which solves the Grad-Shafranov equation using data from primarily magnetic diagnostics as constraints. Additional constraints can be supplied to improve the quality of the magnetic reconstruction. This information is passed to the DG-CARRE code [63], which calculates a quasi-orthogonal mesh on which simulations are carried out.

\subsection{SOL Potentials \& Currents}

\subsubsection{SOL Potentials}

Electrostatic fields arise in the scrape-off layer of Tokamaks as electrons, being highly mobile, charge up surfaces in the vacuum vessel negatively. Consequently, the plasmamaterial interface is influenced by an electrostatic sheath, as described in chapter 2 . Away from solid surfaces in the bulk SOL parallel and radial electrostatic fields drive 
radial and parallel motion respectively via the $\vec{E} \times \vec{B}$ drift. Models describing the effects of the $\vec{E} \times \vec{B}$ drift must first begin with accurate calculations of the electrostatic fields within the plasma. The magnitude of the electrostatic potential in a Tokamak scrape-off layer can be calculated by the electron momentum equation. A simplified form of this equation, described as Ohm's law in [8], is given by:

$$
-\nabla_{\|} \phi=\eta j_{\|}-0.71 \frac{d T_{e}}{d s}-\frac{1}{n_{e}} \frac{d p_{e}}{d s}
$$

where $\nabla_{\|} \phi$ is the derivative of the electrostatic potential along a field line, $j_{\|}$is the parallel current density flowing in the plasma $\left(\mathrm{Am}^{-2}\right), \eta$ is the parallel electric resistivity of the plasma (ohm.m), $T_{e}$ is the electron temperature $(\mathrm{eV}), n_{e}$ is the electron density $\left(\mathrm{m}^{-3}\right), p_{e}$ is the electron pressure $\left(n_{e} T_{e}\right)(\mathrm{Pa})$ and $s$ is the distance along a field line $(\mathrm{m})$. Equation (4.4) facilitates the direct calculation of the electrostatic field via the expression:

$$
E=-\nabla_{\|} \phi
$$

where $E$ is the electrostatic field $\left(\mathrm{Vm}^{-1}\right)$. It is convenient to specify the boundary conditions at the material bounding the plasma, which is either biased (to a known potential) or floating. In the latter case, the following expression can be used to calculate the floating potential at the sheath entrance, $\phi_{s f}(\mathrm{~V})[8]$ :

$$
\frac{\phi_{s f}}{T_{e}}=0.5 \ln \left[\left(2 \pi \frac{m_{e}}{m_{i}}\right)\left(1+\frac{T_{i}}{T_{e}}\right)\right]
$$

where all terms are as defined above. Accurate calculation of the plasma potential requires knowledge of the plasma current profile, $j_{\|}$, which arises due to temperature asymmetries at surfaces bounding magnetic flux tubes, known as thermoelectric currents, and Pfirsch-Schlüter currents [64]. The calculation of the magnitude of these currents, and their effects on the plasma potential, are be elucidated in sections 4.2.2, 4.2.3 and 4.5.2.

\subsubsection{Thermoelectric Currents}

It was postulated by Harbour [65] that electrical currents along field lines in the SOL could be generated naturally by $\mathrm{T}_{e}$ asymmetries at the ends of field lines where they intersect solid surfaces. As the sheath potential close to solid surfaces (equation 4.6) is a function of $\mathrm{T}_{e}$ and $\mathrm{T}_{i}$, temperature differences at the ends of open field lines result in a potential difference between it's ends, with the plasma acting as a conductor between them. Furthermore, an additional contribution to this current can come from electron pressure gradient forces within the plasma. The resultant current driven along field 
lines due to these effects is known as the thermoelectric current. The form of the thermoelectric current, $j_{\|}$, used in this study originates from a derivation by Stangeby [8], which is based on Ohm's law:

$$
e \frac{j_{\|}}{\sigma}=-e \frac{d \phi}{d s}+0.71 \frac{d k T_{e}}{d s}+\frac{1}{n_{e}} \frac{d p_{e}}{d s}
$$

where $\sigma$ is the parallel plasma conductivity, given by $\sigma=1 / \eta$, and all other terms have their normal meanings. The first term on the RHS of (4.7) is to take into account external biasing of one of the surfaces with respect to the other, grounded surface. The second term is due to the effects of ion-electron collisions. The ion-electron collision frequency $\left(\mathrm{s}^{-1}\right)$ for momentum-loss collisions is given by [8]:

$$
\nu_{e i}^{m o m}=\frac{0.51 e^{4} \ln \Lambda Z_{i} n_{e}}{3 m_{e}^{\frac{1}{2}} \epsilon_{o}^{2}\left(2 \pi e T_{e}\right)^{\frac{3}{2}}}
$$

The inverse $\nu_{e i}^{\text {mom }}$ with $\mathrm{T}_{e}$ relationship means that ions suffer a greater collisional drag on their motion from collisions with colder ions than with warmer ions. As a result, momentum-loss collisions between ions and electrons have the effect of pushing ions up electron temperature gradients. The final term is the electron pressure gradient force.

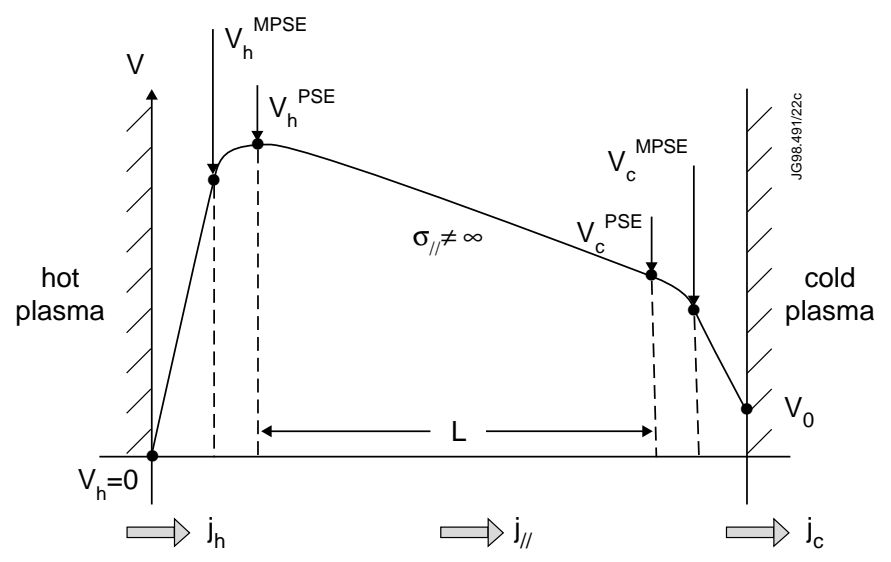

Figure 4.1: Illustration of thermoelectric currents flowing from the higher temperature to the lower temperature target plates from [8].

A full derivation of the thermoelectric current equation is not given here, but integrating (4.7) from the cold plate to the hot plate in figure 4.1 yields [8]: 


$$
\begin{aligned}
\hat{j}_{\|}=-\gamma\left[\frac{e V_{0}}{k T_{h}}\right. & +\left(\frac{1}{r_{T}}-1\right)(\ln 2-0.71+\ln \alpha) \\
& +\ln \left[\frac{1+\hat{j}_{\|}}{\left(1-r_{n} r_{T}^{\frac{1}{2}} \hat{j}_{\|}\right)^{\frac{1}{r_{T}}}}\right]-\frac{1}{k T_{h}} \int_{h}^{c} \frac{1}{n} \frac{d p_{e}}{d s} d s
\end{aligned}
$$

where $r_{T}=\frac{T_{h}}{T_{c}}$ is the ratio of the electron temperatures at the "hot" and "cold" divertor plates, $r_{n}=\frac{n_{h}}{n_{c}}$, analogous to $r_{T}$ but for electron density, $\alpha=\frac{1}{2} \sqrt{\frac{m_{i}}{\pi m_{e}}}, V_{0}$ is the biasing of the "cold" plate with respect to the "hot" one (V), and

$$
\hat{j}_{\|}=\frac{j_{\|}}{e n_{h} c_{s h}}
$$

the thermoelectric current is normalised to the ion saturation current at the "hot" plate,

$$
\begin{gathered}
\gamma=\frac{\bar{\sigma}_{\|} k T_{h}}{e^{2} L_{\|} n_{h} c_{s h}} \\
\bar{\sigma}=\left[\frac{1}{L} \int_{h}^{c} \frac{d s}{\sigma}\right]^{-1}
\end{gathered}
$$

and $L_{\|}$is the connection length (m). Using $n_{e}, T_{e}$ and $T_{i}$ profiles from OSM, the thermoelectric current was calculated along field lines in a grid for MAST shot 13018 at $250 \mathrm{~ms}$ (figure 4.2) [66]. Equation 4.9 was solved using Ridders' method [67] as the derivative of 4.9 with respect to $\hat{j}_{\|}$is not required in this method, which increases computational speed. Furthermore, this method only looks for solutions of 1D nonlinear equations within specified bounds. In the case of solving equation 4.9, this is useful because beyond two limits $\left(\hat{j}_{\|}=-1\right.$ and $\left.\hat{j}_{\|}=\frac{1}{r_{n} \sqrt{r_{T}}}\right)$, the function becomes zero and infinity respectively. All information concerning plasma properties and the magnetic equilibrium (required to calculate the connection length) were taken from a converged OSM solution, whilst calculating the thermoelectic current at each iteration.

The simulation results are compared with measurements of the current density in the SOL as measured by Langmuir probes embedded in the divertor targets. Good agreement is observed between experimental data and the thermoelectric current model, which is consistent with observations made on JT-60U [68], ASDEX-Upgrade [69], COMPASS [70], JET [71] and others. For the case shown here, the dominant driving term for thermoelectric current is the temperature asymmetry at the divertor targets. The magnitude of the pressure gradient dependent term is an order of magnitude smaller than those arising due to target temperature asymmetry. This is not atypical, 

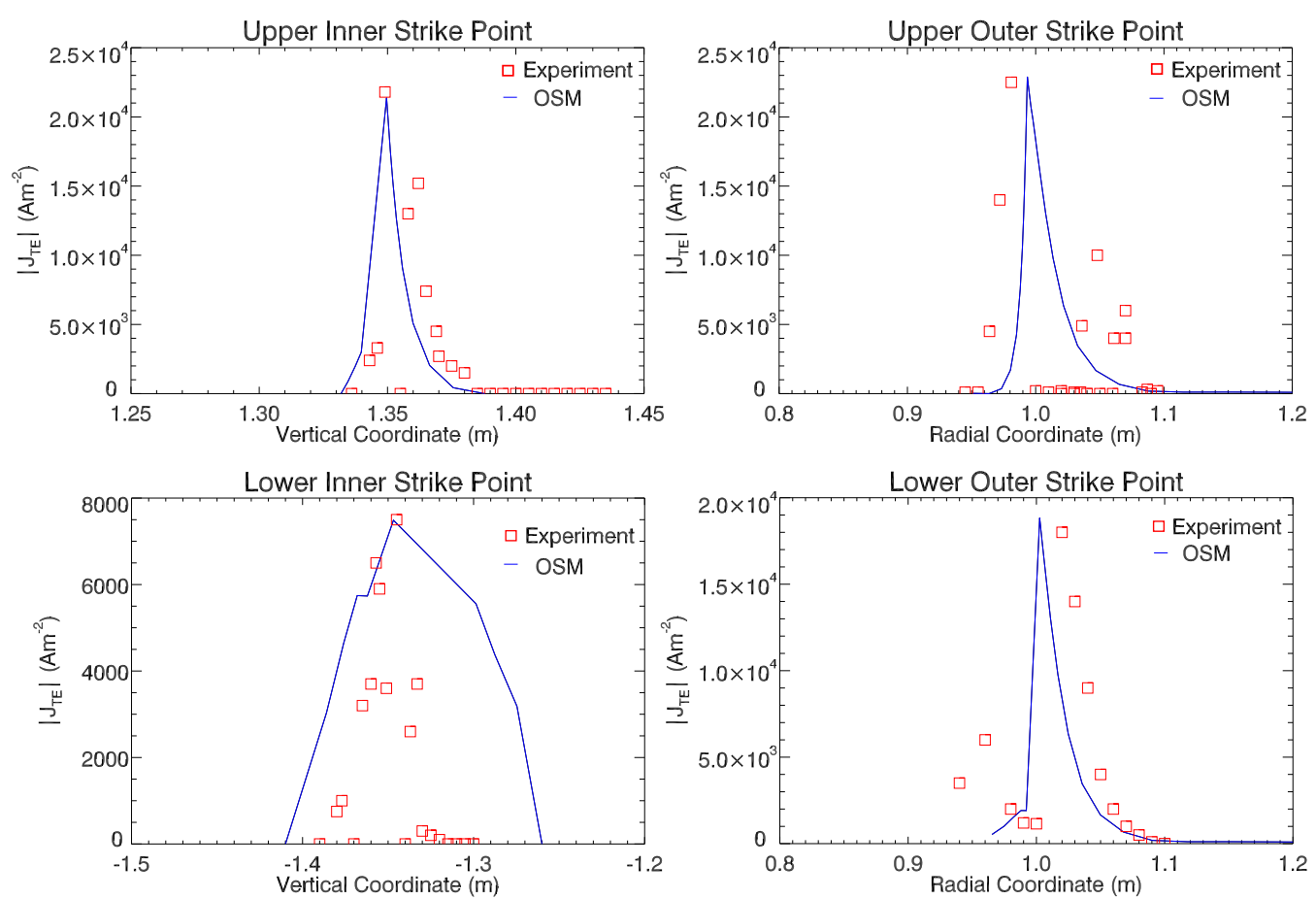

Figure 4.2: Calculated (blue) thermoelectric currents using temperature and density profiles from OSM for MAST shot 13018 at 250ms. Experimentally determined currents at the divertor target plates measured by target Langmuir probes are also shown (red). $[66]$

as observations on JET [71] showed that the pressure gradient term only plays a significant role in the event of small target temperature asymmetries. In the discharge and time modelled in this case, the ratio of the electron temperatures at the hot and cold plates ranged from 1.0 to 5.0. In the former case, the pressure gradient term was approximately one third as large as the temperature asymmetry term, compared to an order of magnitude smaller in the latter case.

\subsubsection{Pfirsch-Schlüter Currents}

The Pfirsch-Schlüter current arises due to the statement of current conservation:

$$
\vec{\nabla} \cdot \vec{j}=0
$$

This states that a divergent current source must be balanced by another current, such that the total current density is divergence free. Therefore, (4.13) can be re-cast in terms of parallel and perpendicular current flows:

$$
\nabla j_{\|}=-\nabla_{\perp} j_{\perp}
$$


The presence of the thermoelectric current suggests that other sources of current must exist in the Tokamak SOL, in order to balance the divergence of parallel and perpendicular currents. Previous studies [64] indicated that the dominant cross-field current source in the SOL is due to diamagnetic drift motion. The specific details of this drift will not be elucidated here, but will in section 4.5.2. The diamagnetic drift velocity is given by:

$$
v_{d i a}=\frac{\vec{B} \times \vec{\nabla} p}{n q B^{2}}
$$

where $\vec{B}$ is the magnetic field vector, $\mathrm{p}$ is the static pressure (i.e. $\mathrm{nT}$ ), $\mathrm{q}$ is the charge of the fluid species of density $\mathrm{n}$. Considering only electrons and a singly ionised deuterium species, the current density due to this drift motion is given by:

$$
j_{d i a}=\left(n_{i} e v_{i, d i a}-n_{e} e v_{e, d i a}\right)
$$

which, combined with (4.15) gives:

$$
j_{d i a}=\frac{\vec{B} \times \vec{\nabla} P}{B^{2}}
$$

where $P=p_{i}+p_{e}$ and $\vec{B}$ is the magnetic field vector. The divergence of this cross-field diamagnetic current is not closed by the thermoelectric current. Therefore, according to (4.14) a parallel current must be invoked in order to satisfy current conservation. A form of this parallel current suited to being incorporated into 1D fluid models was given by Rozhansky [64]:

$$
\begin{aligned}
j_{\|}(x)=j_{\|}\left(x_{0}\right) \frac{B_{T}(x)}{B_{T}\left(x_{0}\right)} & +\frac{B_{T}(x) B(x)}{B_{\theta}(x)}\left(\frac{1}{B^{2}} \frac{d p}{d r}(x)-\frac{1}{B^{2}} \frac{d p}{d r}\left(x_{0}\right)\right) \\
& -\frac{B_{T}(x) B(x)}{B_{\theta}(x)} \frac{d}{d r} \int_{x_{0}}^{x} \frac{1}{B^{2}\left(x^{\prime}\right)} \frac{d p}{d x^{\prime}} d x^{\prime}
\end{aligned}
$$

where $\mathrm{B}_{T}$ is the toroidal field strength $(\mathrm{T}), \mathrm{B}_{\theta}$ is the poloidal field strength $(\mathrm{T}), \mathrm{x}$ is a poloidal coordinate and $\mathrm{x}_{0}$ is a reference poloidal coordinate where the current density $\mathrm{j}_{\|}$is known. In this case, $\mathrm{x}_{0}$ is taken to be one of the divertor plates, as it is found in MAST to resemble the thermoelectric current, as shown in the previous section. This is a useful boundary condition in the calculation of $\mathrm{j}_{\|}$, as the thermoelectric current can be calculated independently. The total current density $j_{\|}$was calculated for the same MAST shot as for the thermoelectric current calculation. The result of the calculation of $j_{\|}$is shown in figure 4.3. The parallel current is seen to reverse direction at the outboard midplane due to an increase in the integrated poloidal pressure gradient term, which is larger on the outboard side. This feature of the calculation was also observed 
by Rozhansky [64].
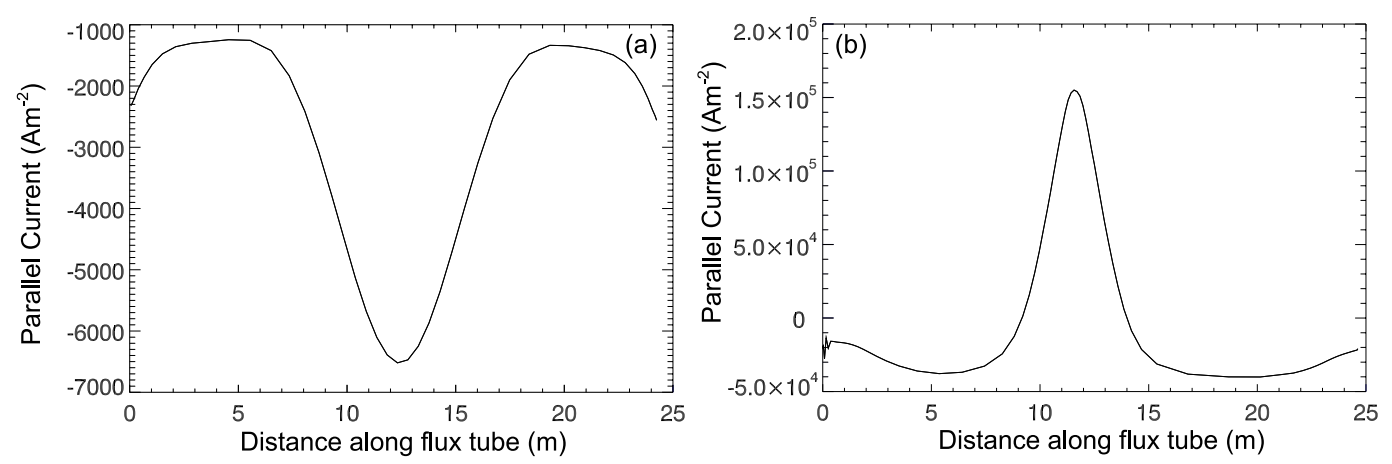

Figure 4.3: Results of the calculation of $\mathrm{j}_{\|}$including thermoelectric and Pfirsch-Schlüter contributions. a) Calculated $\mathbf{j}_{\|} 1 \mathrm{~cm}$ inboard of the separatrix on the high-field side $\mathrm{b}$ ) $\mathrm{j}_{\|} 1 \mathrm{~cm}$ outboard of the separatrix on the low-field side. [66] In this geometry, on the left graph, $\mathrm{s}=0 \mathrm{~m}$ is the position of the lower inner strike point and $\mathrm{s}=25 \mathrm{~m}$ is the location of the upper inner strike point. On the right graph, $s=0$ is the location of the upper outer strike point and $\mathrm{s}=25 \mathrm{~m}$ is the location of the lower outer strike point.

\subsection{Flux Expansion Terms}

The importance of terms in the fluid conservation equations relating to the effects of varying cross-sectional area of magnetic flux tubes [72], also referred to as the magnetic mirror force, was highlighted in a study by Kirk [73]. In this study, it was found that terms describing the effects of flux expansion were up to 10 times greater in magnitude in MAST compared to JET. Consequently, the additional transport terms arising from flux expansion had a significant effect on the plasma solution, demonstrated by performing code runs with and without these terms.

As a result, these flux expansion terms were incorporated into the OSM model used in this study to better refine the transport model. By default, OSM does not include information on the total magnetic field strength, only the ratio of the poloidal and total field strengths is stored, as this is used to calculate the field line pitch angle, which is required to calculate the along-field distance, s. Therefore, the equilibrium fitting code EFIT [62], from which magnetic field components can be calculated (figure 4.4), was coupled with OSM to provide additional information about the magnetic field and it's components. By default, EFIT calculations are carried out on a $65 \times 65$ rectangular grid, so the computed magnetic field strength was calculated on the EFIT mesh, then passed to OSM for interpolation onto the modelling grid in use. The interpolation was carried out using cubic splines in two dimensions, with an algorithm explained in [67]. To check the EFIT calculation and interpolation were consistent with the modelling grid, the ratio of the poloidal and total magnetic fields were also passed to OSM and 

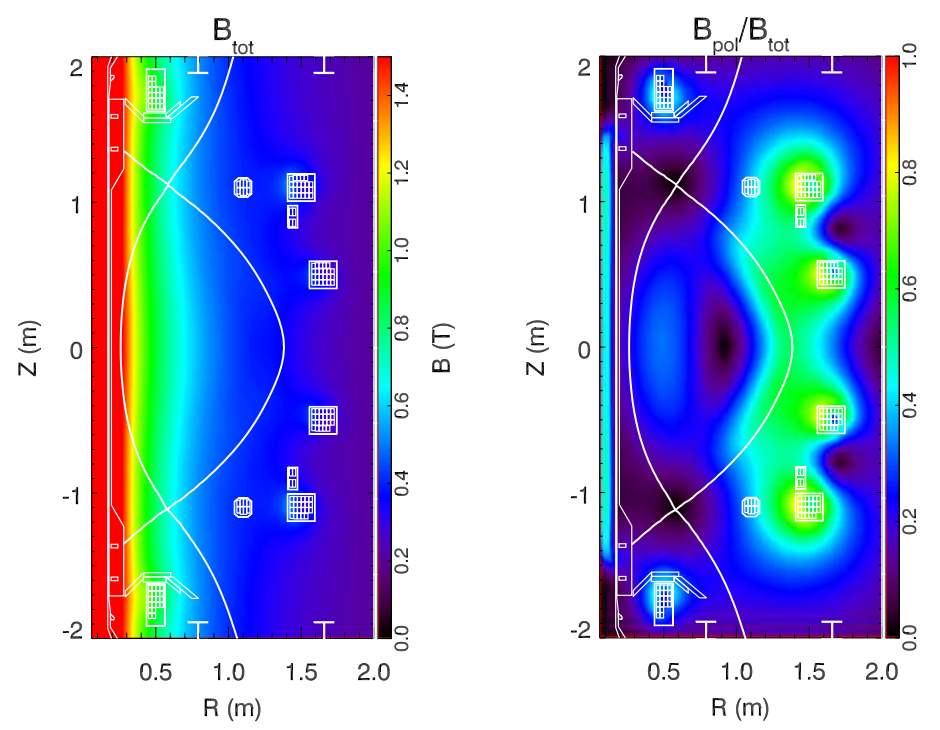

Figure 4.4: Left: the total magnetic field strength in a typical MAST plasma with the plasma boundary overlaid. Right: the ratio of the poloidal and total magnetic field strengths calculated by EFIT.

interpolated onto the modelling grid, for comparison with the values passed to the code in the original grid file. Good agreement was found (typically a $2 \%$ deviation) between the two values.

The key step in the derivation of these terms is moving from scalar conservation equations, such as that of particle conservation:

$$
\frac{\partial\left(n u_{\|}\right)}{\partial s}=S_{p}
$$

into a vector equation:

$$
\vec{\nabla} \cdot\left(n u_{\|} \vec{b}\right)=S_{p}
$$

where $\vec{b}$ is a unit vector pointing along the magnetic field line. The difference between these equations become apparent when 4.20 is expanded to give:

$$
\frac{\partial\left(n u_{\|}\right)}{\partial s}-\frac{\nabla_{\|} B}{B} n u_{\|}=S_{p}
$$

using:

$$
\vec{\nabla} \cdot \vec{b}=-\frac{\nabla_{\|} B}{B}
$$

where $\nabla_{\|}=\hat{b} \cdot \vec{\nabla}$. There are also additional terms in the momentum conservation equation arising from this effect, that are re-derived in a similar manner as for the 
particle conservation equation, yielding:

$$
\frac{\partial}{\partial s}\left(n_{e} T_{e}+n_{i} T_{i}+m_{i} v_{i}^{2}\right)-\frac{\nabla_{\|} B}{B} m_{i} v_{i}^{2}=S_{m}
$$

Conveniently, the corrections to the conservation equations that arise due to flux expansion effects appear as lower-order terms from those they originated from, i.e. the corrections to convective terms appear as sources. This allows these additional terms to be easily incorporated into numerical schemes.
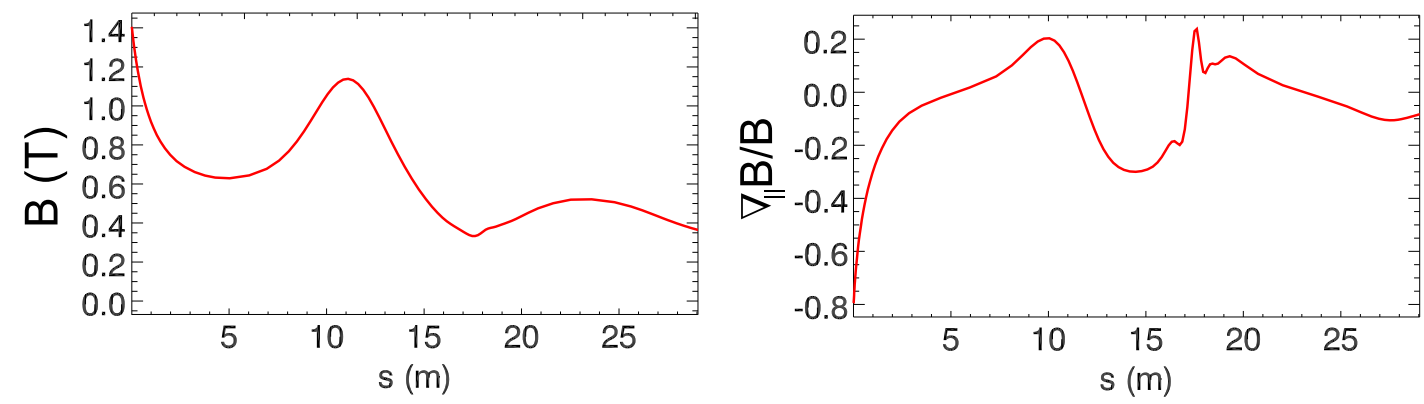

Figure 4.5: Left: the total magnetic field strength measured along a flux tube in a lower single-null discharge. Right: Calculation of $\nabla_{\|} B / B$ along the same flux tube. In this geometry, $\mathrm{s}=0 \mathrm{~m}$ is the location of the lower inner strike point and $\mathrm{s} \approx 29 \mathrm{~m}$ is the location of the lower outer strike point.

The effects of the flux expansion terms are explored by examining data from a partially detached MAST discharge, shot number 24861 at $240 \mathrm{~ms}$. This shot was in a lower single-null geometry, which is brought about by shifting the plasma downwards. $1 \mathrm{MW}$ of auxiliary heating was provided by a neutral beam injector. The lower inner divertor target was partially detached by puffing $\mathrm{D}_{2}$ gas in a region close to the lower inner strike point. The variation in the strength of the flux expansion terms can be inferred from the variation of $\nabla_{\|} B / B$ along a field line (figure 4.5). In order to judge the effects of these additional terms, a comparison is made of the OSM plasma solutions with the terms active and inactive in the code.

To test whether the additional terms had been implemented correctly, the flux expansion terms were included as part of the left hand-side of the conservation equation using a Runge-Kutta solver running OSM without EIRENE. This allows direct comparison of the results from the numerical schemes without undue complexity. The results were compared to results from the original OSM solver, where the additional terms were included by treating them as sources (at the right hand-side of the conservation equations) and iterating the solver until convergence was reached (figure 4.7). Both numerical schemes agree very well, indicating that implementation of the additional terms is equally acceptable. For the remainder of this section, the calculations carried out use the Runge-Kutta OSM solver and the effects of flux expansion terms are 


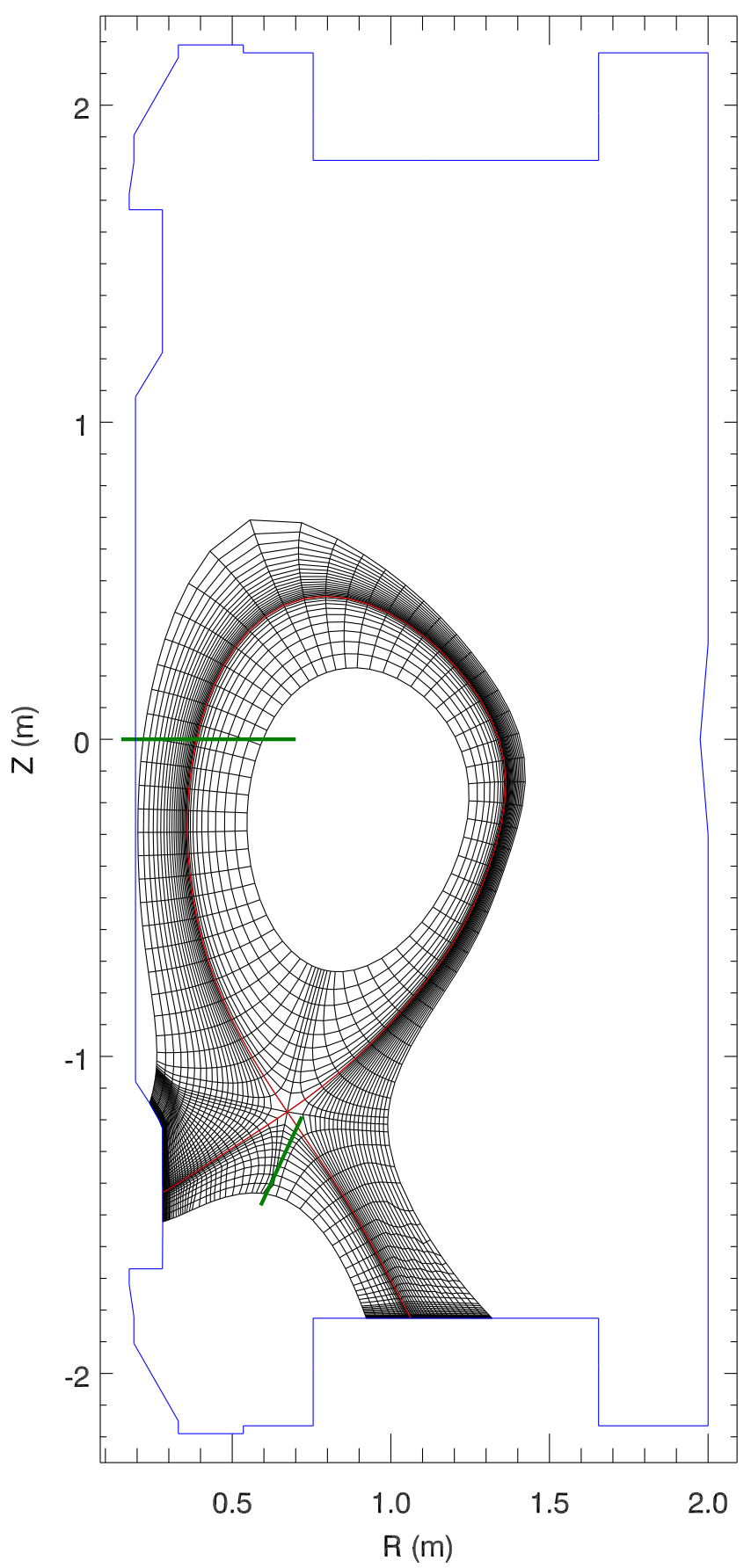

Figure 4.6: Magnetic grid used in OSM-EIRENE modelling of MAST discharge 24861 at $240 \mathrm{~ms}$. The green line at $\mathrm{z}=0$ represents the domain over which electron density and temperature were specified using Thomson scattering. The additional green line is used to specify conditions within the private flux region. Ion fluxes to the divertor targets are also specified using data from Langmuir probes embedded in the target plates. 

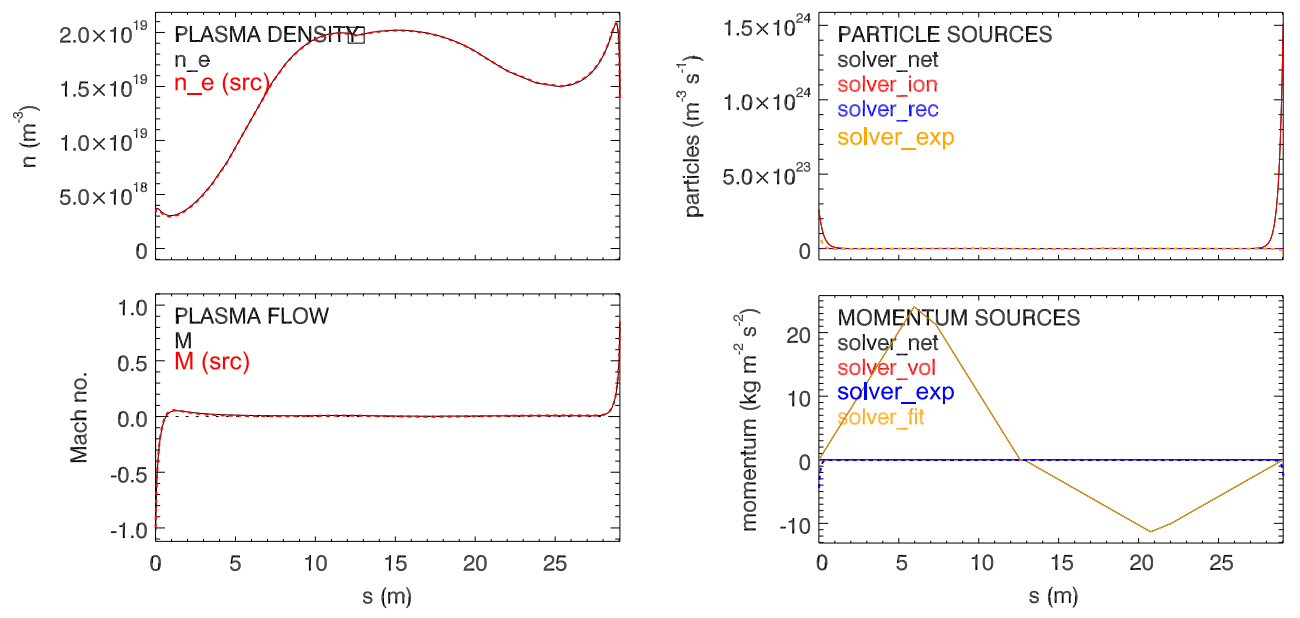

Figure 4.7: Results from two OSM runs, the dashed lines indicate data taken from when the flux expansion terms were expressed as sources, using the original OSM solver. The solid lines indicate data from a run where flux expansion terms were part of the conservation equations in a Runge-Kutta solver. Electron temperature profiles were omitted, as they were interpolated from experimental data and hence not calculated in the numerical solvers for $\mathrm{n}_{e}, \mathrm{v}_{i}$.

included as part of the conservation equation.

In the previous example, the effects of the additional terms, reflected by the magnitude of the particle and momentum sources they bring about, is small compared to other sources used in the code. However, in this test case, the particle sources due to ionisation of neutrals was computed such that ion flow in the bulk of the SOL was low. As the flux expansion correction terms are both functions of the ion velocity, this directly influences the magnitude of these terms. To properly assess the importance of these terms, OSM-EIRENE runs were carried out, which calculates realistic ionisation sources based on atomic physics, and hence does not yield solutions with low ion flow in the SOL. Figure 4.8 shows the results of an OSM-EIRENE calculation, iterated with calculated particle and momentum sources arising from the flux expansion terms. A striking feature of OSM runs on MAST is the pressure imbalance between upstream locations and the divertor targets. This is a common feature of parallel scrape-off layer profiles in attached MAST discharges that this work will help address.

In terms of particle balance, the flux expansion sources are relatively modest (figure 4.9) and only dominate over the other sources in the vicinity of the outboard midplane and are comparatively strong in the vicinity of the inner divertor plate. Both of these locations correspond to regions of high $\nabla_{\|} \mathrm{B} / \mathrm{B}$ (figure 4.5), indicating the importance of this term in the flux expansion correction terms.

The OSM-EIRENE solution, which includes flux expansion correction terms, still has modest parallel flows (and hence parallel flux) in the bulk of the scrape-off layer, 

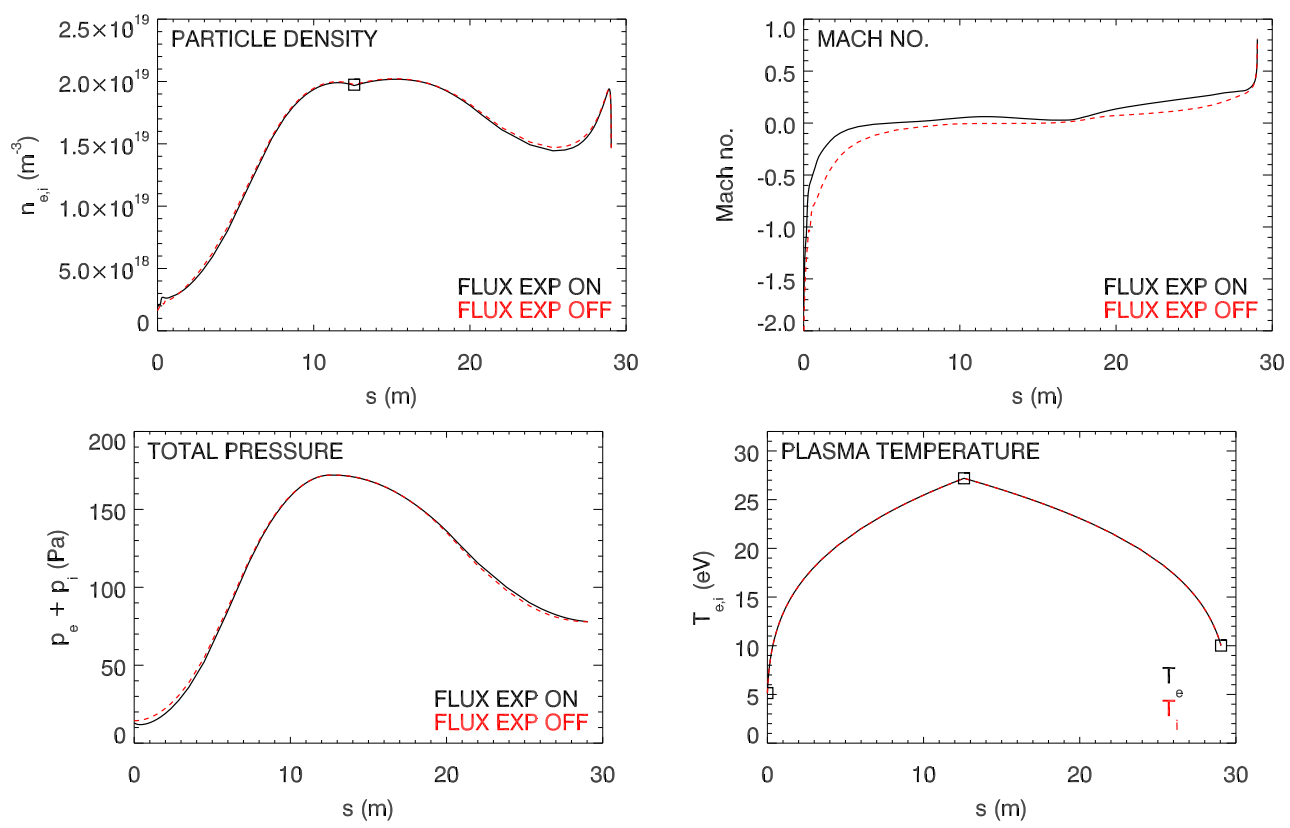

Figure 4.8: Results from an OSM-EIRENE run with flux expansion correction terms. The points highlighted with squares indicate where diagnostic information has been provided, either by target Langmuir probes or Thomson scattering in this case.

compared to measurements on other devices that suggest that ion flow in the bulk of the SOL is $\approx 0.5$ [74]. This indicates that these terms are significant even when the ion flux may be underestimated. It is difficult to make a direct comparison of the plasma density and parallel ion velocity with and without the correction terms on, as the OSM fitting source term (solver_fit in figure 4.9) varies to ensure that the plasma solution agrees with the experimental data used as boundary conditions in the code. Therefore, a useful comparison is the effect of the flux expansion terms on solver fit. OSM-EIRENE runs with and without the flux expansion terms (figure 4.9) show that solver fit (purple trace) is smaller when the terms are applied compared to when they are not. This indicates that the numerical scheme needs to apply a smaller correction to the conservation equations to make the plasma solution match the supplied experimental data, by almost an order of magnitude.

The effects of the flux expansion terms in the momentum equation (figure 4.10) are localised to the regions close to the divertor targets due to the correction term being proportional to the square of the parallel ion velocity in this case, and linear in terms of $\nabla_{\|} B / B$. However, the correction term is larger at the inner strike point, where $\nabla_{\|} B / B$ is greatest. As the correction term is strongest at the locations where the OSM fitting source is smallest, the effect on this source overall is low.

Finally, it is interesting to observe the effects of flux expansion correction terms in the calculation of the electrostatic potential. Taking expressions for the parallel 


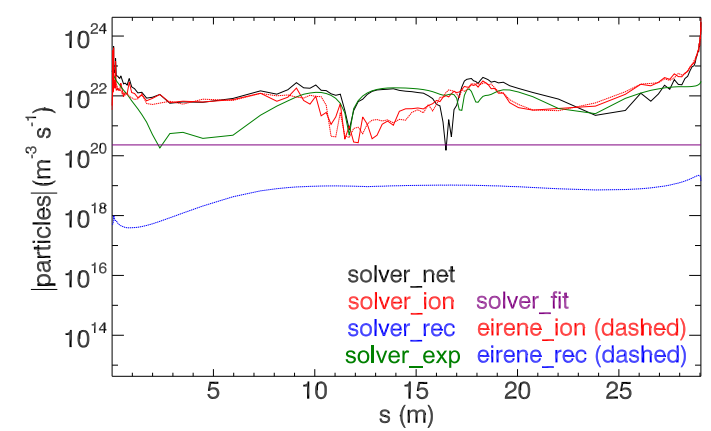

(a) Particle sources with flux expansion terms.

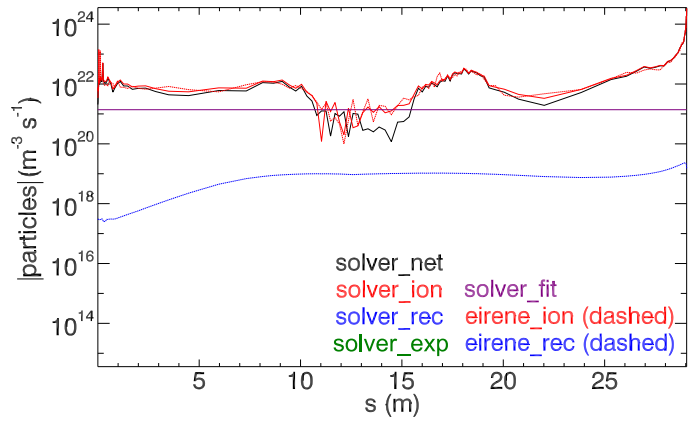

(b) Particle sources without flux expansion terms.

Figure 4.9: The distribution of particle sources along the separatrix flux tube in MAST shot 24861. In this simulation, solver_exp (green) is the particle source term arising due to extra terms in the particle conservation equation due to magnetic field variations. solver_ion (red) and solver_rec (blue) are particle sources and sinks arising due to ionisation and recombination reactions, respectively. The dashed curves indicate EIRENE sources and sinks from a previous code iteration, to check for convergence. Note that the y-axis indicates the modulus of the particle source, as this axis has a logarithmic scale.

component of Ohm's law and current conservation, assuming no cross-field sources of current, one gets:

$$
\begin{gathered}
-\nabla_{\|} \phi=\eta_{\|} j_{\|}-\frac{0.71}{e} \frac{d k T_{e}}{d s}-\frac{1}{e n_{e}} \frac{d p_{e}}{d s} \\
B \nabla_{\|}\left(\frac{j_{\|}}{B}\right)=0
\end{gathered}
$$

Expressions (4.24) and (4.25) can be combined to form one second-order ODE:

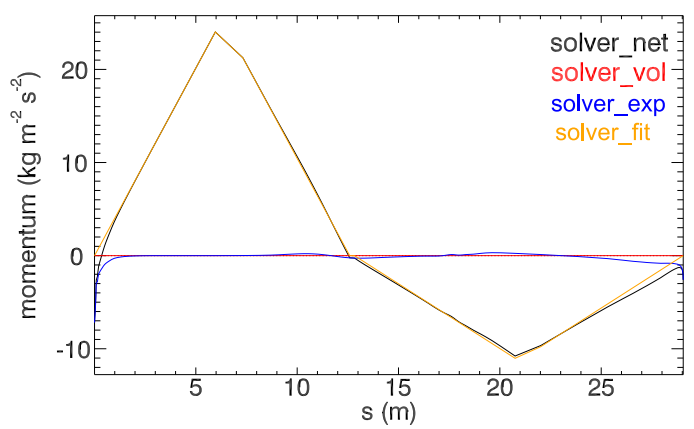

(a) Momentum sources with flux expansion terms.

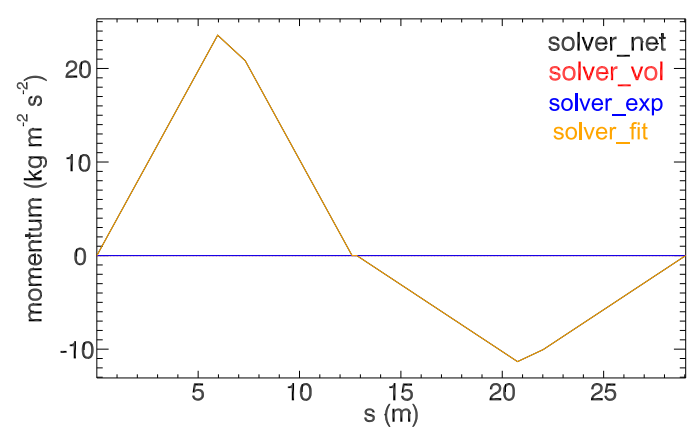

(b) Momentum sources without flux expansion terms.

Figure 4.10: The distribution of momentum sources in MAST shot 24861. The momentum source due to the flux expansion terms is given by solver_exp (blue). 

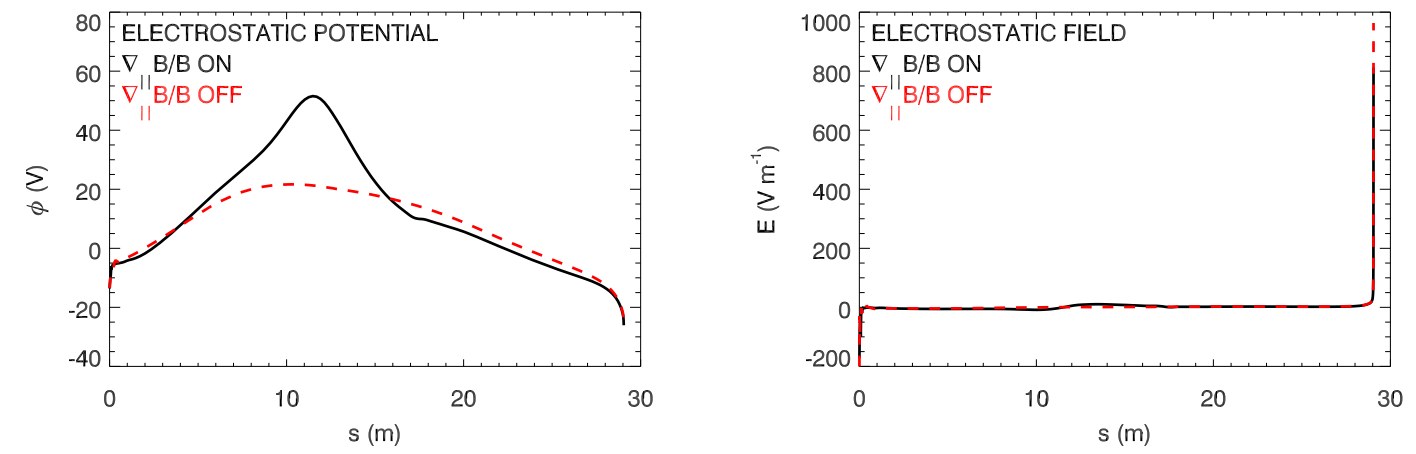

Figure 4.11: Effects of parallel magnetic field gradient terms on the calculated electrostatic potential and electrostatic field.

$$
\begin{aligned}
\nabla_{\|}^{2} \phi-\left(\frac{1}{\eta} \nabla_{\|} \eta+\frac{1}{B} \nabla_{\|} B\right) \nabla_{\|} \phi= & 0.71 \nabla_{\|}^{2} T_{e}-0.71 \nabla_{\|} T e\left(\frac{\nabla_{\|} B}{B}\right)-\frac{0.71}{\eta} \nabla_{\|} T e \nabla_{\|} \eta \\
& +\frac{1}{n_{e}} \nabla_{\|}^{2} p_{e}-\frac{1}{n_{e}^{2}} \nabla_{\|} p_{e} \nabla_{\|} n_{e}-\frac{1}{\eta n_{e}} \nabla_{\|} p_{e} \nabla_{\|} \eta \\
& -\frac{1}{n_{e}} \nabla_{\|} p_{e}\left(\frac{\nabla_{\|} B}{B}\right)
\end{aligned}
$$

Three terms in (4.26) are proportional to $\frac{\nabla_{\|} B}{B}$, which can significantly alter the calculated potential distribution in the SOL (figure 4.11). A peak in the plasma potential has been created by a peak in $\nabla_{\|} B / B$ at $\mathrm{s} \approx 10 \mathrm{~m}$, which roughly corresponds to the location of the inboard midplane. Consequently, there exists a non-zero electrostatic field in the vicinity of the peak.

\subsection{Parallel Viscosity}

An important step in the derivation of plasma fluid models is how a closed set of fluid equations is obtained, which normally involves making assumptions about the level of plasma collisionality. Terms relating to parallel viscosity arise from a fluid closure scheme as described in a derivation of plasma fluid equations by Zawaideh [75], which are used in OSM and are valid for highly collisional and collionless plasma conditions. As the name implies, collisionality refers to how collisions between different plasma species affect plasma transport. The word "collision" refers to very shortrange encounters between charged particles and the more frequent, but less strong, electromagnetic interactions between more distant charged particles. When deriving the fluid energy conservation equation, one is left with two unknowns, the pressure in the parallel (along field lines) and perpendicular pressures, denoted $\mathrm{p}_{\|}$and $\mathrm{p}_{\perp}$ 
(and, by extension, parallel and perpendicular temperatures). Parallel viscosity can be introduced to prevent taking higher-order moments of the kinetic equation by definition:

$$
\pi=\frac{2}{3}\left(p_{\|}-p_{\perp}\right)
$$

where $\pi$ is the parallel stress tensor. In this study, the ion component of the parallel stress tensor retained after dropping small terms (terms including $\pi[8]$ ) is [75]:

$$
\pi_{i}=-\frac{4}{9} p_{i} \tau_{\perp \rightarrow \|} \frac{d v_{\|}}{d s}=-\eta_{\|} \frac{d v_{\|}}{d s}
$$

where $\mathrm{p}_{i}$ is the ion static pressure $(\mathrm{Pa}), \tau_{\perp \rightarrow \|}$ is taken to be the ion-ion collision time (s) and $\eta_{\|}$is the parallel viscosity coefficient, defined in (4.28), not to be confused with the plasma electrical resistivity $\eta$ in (4.4). Only the ion component of the parallel stress tensor is retained as electrons are significantly more collisional than ions (i.e. small $\left.\tau_{\perp \rightarrow \|}\right)$, thereby being a small contribution to $\pi\left(\pi=\pi_{i}+\pi_{e}\right)$ [72]. In the OSM model, parallel viscosity manifests as an additional transport term in the momentum equation, as viscosity is an explicit function of pressure. The parallel momentum conservation including the effects of parallel viscosity is given by:

$$
\frac{d}{d s}\left(n_{e} T_{e}+n_{i} T_{i}+m_{i} n_{i} v_{i}^{2}-\eta_{\|} \frac{d v_{i}}{d s}\right)=S_{m}
$$

The negative sign of $\eta_{\|}$and the dependence on the parallel velocity gradient have the effect of smoothing velocity gradients in the plasma solution. The effects of this term are particularly pronounced at the plasma-surface interface where the plasma ions accelerate to their acoustic velocity to satisfy the Bohm criterion. An additional refinement of the model can be made by incorporating the additional terms of section 4.3 , together with an additional term due to a flux expansion correction to parallel viscosity:

$$
\frac{\partial}{\partial s}\left(n_{e} T_{e}+n_{i} T_{i}+m_{i} v_{i}^{2}-\eta_{\|} \frac{\partial v}{\partial s}+\frac{\nabla_{\|} B}{B} \eta_{\|} v\right)-\frac{\nabla_{\|} B}{B} m_{i} v_{i}^{2}=S_{m}
$$

The effects of the additional transport terms arising from parallel viscosity are ascertained, as before, by running OSM-EIRENE with and without the terms being present in the conservation equations. In order to simplify the analysis, the comparison made here is between an OSM run with the original model (i.e. no flux expansion terms) and (4.30). As before, OSM-EIRENE was run with data taken from shot 24861 at $240 \mathrm{~ms}$ using the same magnetic geometry as that used in section 4.3 . Unlike before, however, this comparison was made using the TVD-MUSCL time-dependent numerical solver, as (4.30) is a convection-diffusion equation, which is best solved with a numerical 

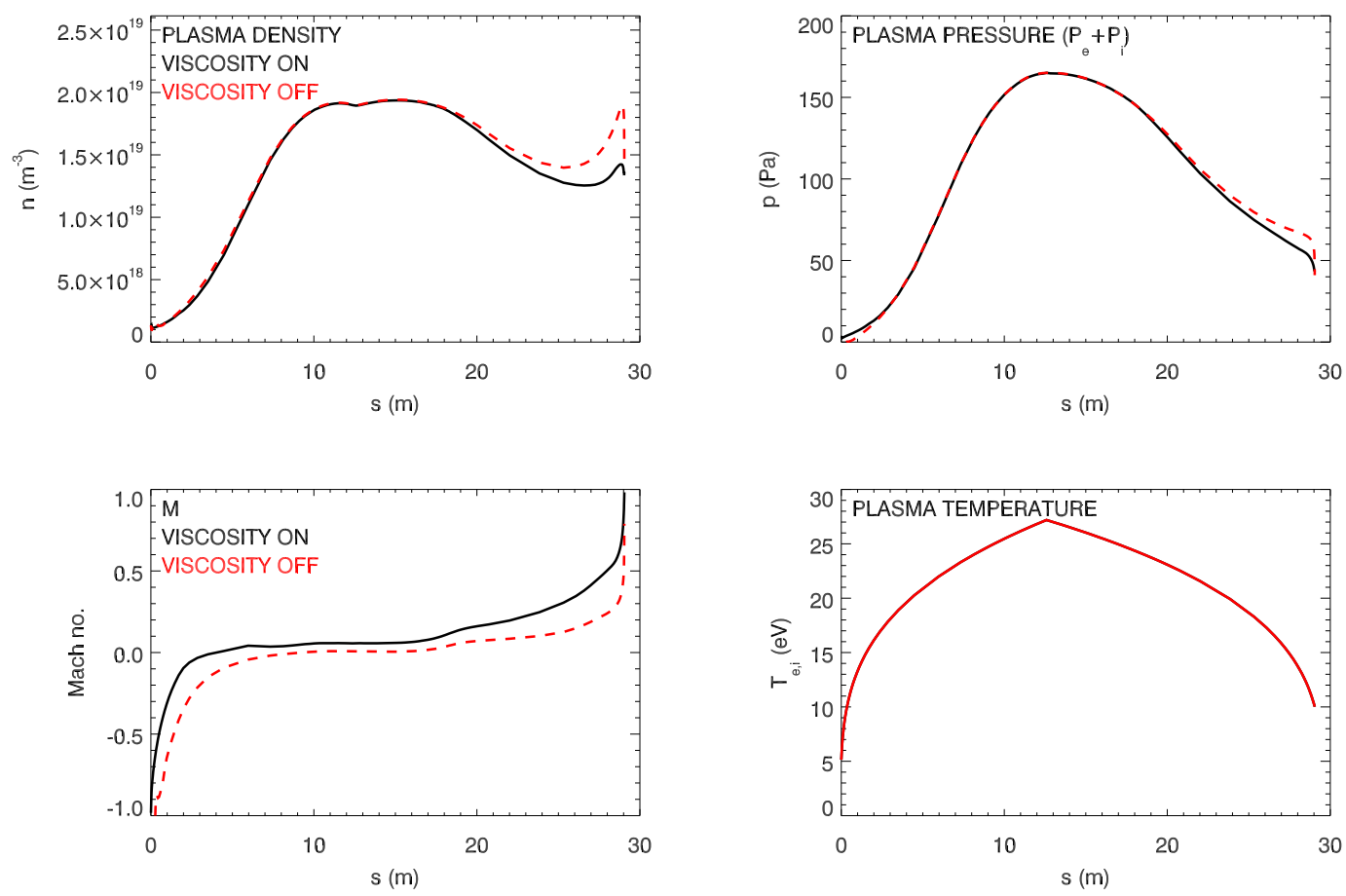

Figure 4.12: The effects of dynamic viscosity on the separatrix ring of MAST discharge 24861 at $240 \mathrm{~ms}$. A significant effect is observed at the outer strike point where a smoothing of the velocity profile has taken place, leading to higher ion velocities in the outer divertor leg. In turn, this has led to a reduction of the density at the outer leg and a consequent reduction in static pressure.

scheme such as this.

The results of the code runs (figure 4.12) indicate a drop in plasma pressure at the outer strike point (high s) due to a drop in density. This is a result of the parallel viscosity term reducing the steep gradient in parallel ion velocity in proximity to the target plate by raising the velocity in the bulk of the SOL compared to the non-viscous case. The pressure at the inboard strike point $(\mathrm{s}=0)$ is slightly raised by the additional transport terms, although the effect on the plasma density is less pronounced than at the outboard side, as $\pi_{i}$ is lower at the inner target.

An important consideration when discussing the application of parallel viscosity is whether or not the plasma conditions under consideration are suitable for the model being applied. In low collisionality conditions, $\tau_{\perp \rightarrow \|} \rightarrow \infty$ and hence $\pi_{i} \rightarrow \infty$, which is unphysical. To prevent this behaviour, kinetic models have suggested [8] a limit of $\left|\pi_{i}\right|<0.5 p_{i}$ be enforced. For the OSM-EIRENE run where parallel viscosity was employed, it is found (figure 4.13) that $\pi_{i}$ never exceeds $0.5 \times \mathrm{p}_{i}$, and so no limit was applied to $\pi_{i}$ in this case.

A comparison of the particle sources used in the OSM-EIRENE code runs (figure 4.14) suggests that a reduction in solver_fit is brought about by the introduction of the 


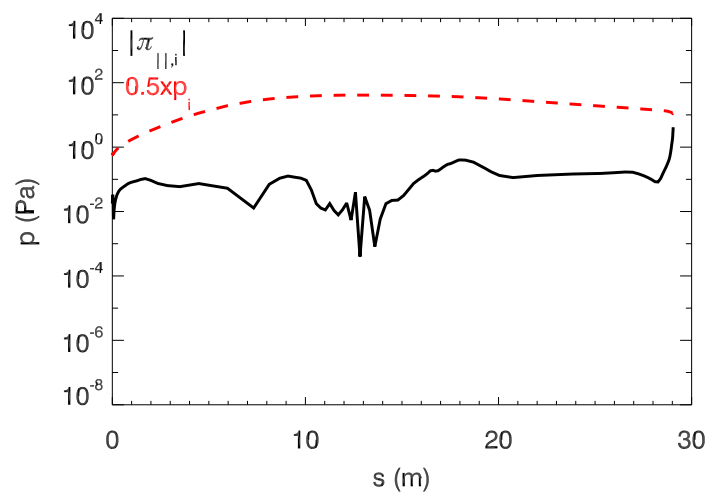

Figure 4.13: A comparison of the ion parallel stress tensor component, $\pi_{i}$, and $0.5 \times$ $\mathrm{p}_{i}$, the ion pressure. Kinetic modelling [8] suggests that $\left|\pi_{i}\right|<0.5 \times p_{i}$, which is automatically satisfied in this case.

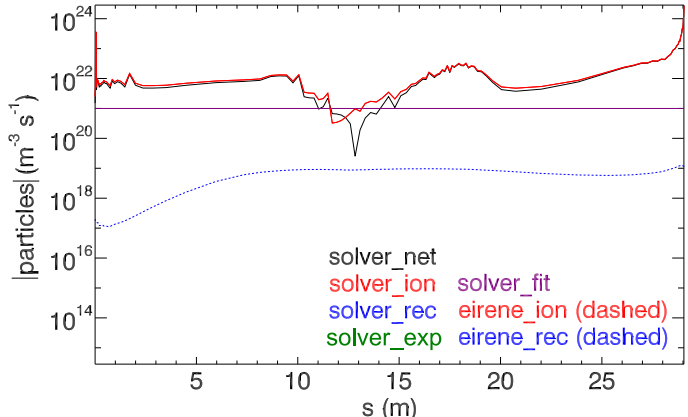

(a) Distribution of particle sources with parallel viscosity included in the conservation equations.

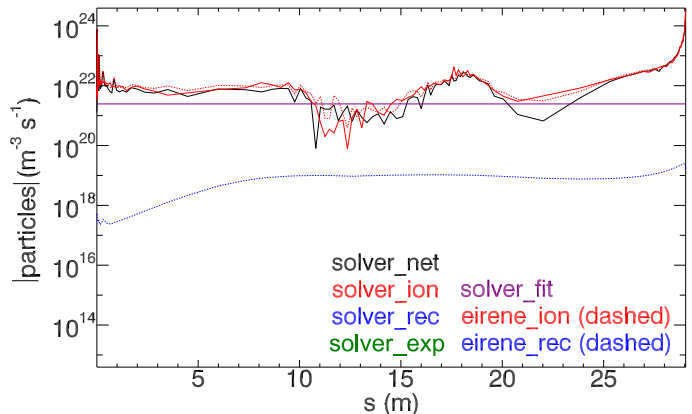

(b) Distribution of particle sources without parallel viscosity included in the parallel conservation equations.

Figure 4.14: Comparison of calculated OSM-EIRENE particle sources (a) with and (b) without parallel viscosity terms invoked. The introduction of the additional transport terms reduces solver_fit, indicating that the numerical scheme requires a weaker fitting particle source for the solution to converge to imposed boundary conditions from experimental measurements. 
parallel viscosity transport terms. A significant proportion $(>90 \%)$ of this reduction was brought about by the $-\frac{d}{d s}\left(\eta_{\|} \frac{d v}{d s}\right)$ term in (4.30), indicating that the $\frac{d}{d s}\left(\frac{\nabla_{\|} B}{B} \eta_{\|} v\right)$ is a less significant correction term.

\subsection{Cross-Field Drifts}

Elementary plasma theory states that if a charged particle has a force exerted upon it which has a component normal to the magnetic lines of force, that particle will gain an additional velocity component perpendicular to that direction, i.e. "drifting" across field lines [76]. This idea extends to the fluid description of fluid behaviour, although not with a 1:1 mapping between single-particle and fluid drift terms. In the scrape-off layer of Tokamaks, cross-field drift motion has been found to explain asymmetries in particle and power fluxes to inner and outer divertor strike points [8,77-79] during experiments where the toroidal magnetic field has been reversed. During such experiments, it has also been noted that scrape-off layer flows are strongly affected $[74,80,81]$ due to parallel flows induced by poloidal and radial drifts.

In the fluid description of plasma transport, the two principal sources of drift motion are $\vec{E} \times \vec{B}$ and diamagnetic [76]. These processes give rise to drift velocities given by:

$$
\begin{gathered}
v_{d, \vec{E} \times \vec{B}}=\frac{\vec{E} \times \vec{B}}{B^{2}}=\frac{\vec{B} \times \nabla \phi}{B^{2}} \\
v_{d, d i a}=\frac{\vec{B} \times \nabla p_{e, i}}{q_{e, i} n_{e, i} B^{2}}
\end{gathered}
$$

where $\vec{E}$ and $\vec{B}$ are the electrostatic and magnetic field vectors $\left(\mathrm{Vm}^{-1}, \mathrm{~T}\right)$ respectively, $\phi$ is the electrostatic potential $(\mathrm{V}), p_{e, i}$ is the static (electron or ion) species pressure $(\mathrm{Pa}), q_{e, i}$ is the species charge $(\mathrm{C})$ and $n_{e, i}$ is the species density $\left(\mathrm{m}^{-3}\right)$.

The $\vec{E} \times \vec{B}$ drift arises from radial and poloidal electrostatic fields driving the motion of ions and electrons along and across magnetic field lines respectively. The diamagnetic drift is a fluid description specific drift term related to the $\nabla B$ and curvature drifts in the single-particle description of plasma behaviour. As implied by equation (4.31), the diamagnetic drift velocity, unlike $\vec{E} \times \vec{B}$, is charge dependent, and so gives rise to an electrical current. This is known as the Pfirsch-Schlüter current, referred to earlier in this chapter.

Other drift transport terms also exist in the fluid description, such as the polarization and centrifugal drifts as the plasma solutions calculated assume steady-state plasma conditions in the former case, and is not thought to play a significant role in scrape-off layer transport in the latter case [82].

With the introduction of a numerical scheme to solve for the electrostatic potential 
profile along field lines in section 4.2 .1 and plasma solvers for $\mathrm{n}_{e, i}, \mathrm{~T}_{e, i}$ and $\mathrm{v}_{\|, i}$, it is possible to incorporate the effects of cross-field drift motion into the OSM transport model.

\section{TOROIDAL PLANE}

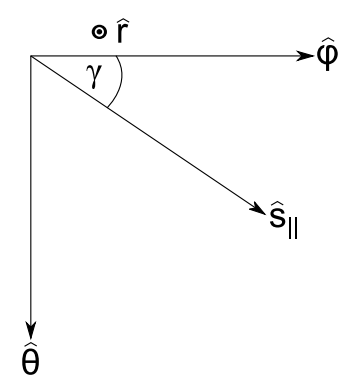

POLOIDAL PLANE

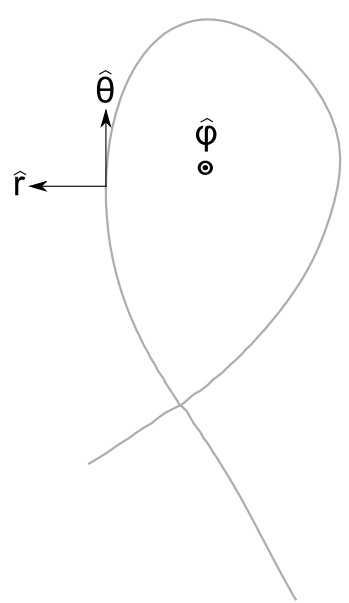

Figure 4.15: The coordinate system used in OSM drift analysis.

The geometry used to calculate the drift transport terms is described in figure 4.15. In OSM, the primary coordinate is the distance along magnetic field lines, $\hat{s}_{\|}$. In order to incorporate $2-\mathrm{D}$ drift motion, the drift terms were calculated using the poloidal $\hat{\theta}$, radial (cross-field) $\hat{r}$ and toroidal $\hat{\phi}$ coordinates (distances). These coordinates form an orthogonal basis which facilitates the calculation of drift velocities and fluxes. In this model, toroidal symmetry is assumed, so that no gradients in any quantities exist in the toroidal $\hat{\phi}$ direction. The effects of drift motion are incorporated in the OSM numerical scheme by calculating particle and momentum sources brought about by the drift motion. These sources are then incorporated in the numerical schemes to solve the particle and momentum conservation equations, and new sources are calculated, and the process iterates until convergence is reached. The process of calculating the relevant sources is explained in the following sections.

\subsubsection{The $\mathrm{E} \times \mathrm{B}$ Drift}

The particle conservation equation for the electron species can be modified to incorporate cross-field drift motion in a straightforward manner $[8,83]$ :

$$
\vec{\nabla} \cdot\left(n_{e} v_{\|, i} \hat{b}-\frac{j_{\|} \hat{b}}{e}+n_{e} \vec{v}_{d}\right)=S_{p}
$$

where: 


$$
v_{e} \hat{b}=v_{\|, i} \hat{b}-\frac{j_{\|} \hat{b}}{e}
$$

therefore, a particle source $S_{p, d}$ can be calculated from an electron drift flux $n_{e} v_{d}$ by:

$$
S_{p, \vec{E} \times \vec{B}}=-\vec{\nabla} \cdot n_{e} \vec{v}_{d}
$$

which can be expanded in the $\{\hat{r}, \hat{\theta}, \hat{\phi}\}$ coordinate system:

$$
S_{p, \vec{E} \times \vec{B}}=-\frac{\partial}{\partial \hat{r}}\left(-B_{\phi} \frac{\partial \phi}{\partial \hat{\theta}}\right)-\frac{\partial}{\partial \hat{\theta}}\left(B_{\phi} \frac{\partial \phi}{\partial \hat{r}}\right)-\frac{\partial}{\partial \hat{\phi}}\left(-B_{\theta} \frac{\partial \phi}{\partial \hat{r}}\right)
$$

where the final term in (4.36) can be neglected due to toroidal symmetry. The final step in the calculation is to calculate the projection of the poloidal component of the drift source in the $\hat{s}_{\|}$direction to allow for consistency with the OSM numerical scheme, which uses $\hat{s}_{\|}$as it's primary coordinate. This is achieved by referring to figure 4.15 :

$$
\hat{\theta}=\hat{s}_{\|} \sin \gamma
$$

where:

$$
\sin \gamma=\frac{B_{\theta}}{|B|}
$$

resulting in the total drift particle source:

$$
S_{p, \vec{E} \times \vec{B}}=\frac{\partial}{\partial \hat{r}}\left(B_{\phi} \frac{\partial \phi}{\partial \hat{\theta}}\right)-\frac{\partial}{\partial \hat{s}_{\|}}\left(\frac{|B| B_{\phi}}{B_{\theta}} \frac{\partial \phi}{\partial \hat{r}}\right)
$$

A similar analysis can be applied to the parallel ion momentum equation:

$$
\vec{\nabla} \cdot\left(m_{i} n_{i} v_{\|, i}\left(v_{\|, i} \hat{b}+\vec{v}_{d}\right)\right)=S_{m}
$$

yielding a drift momentum source:

$$
S_{m, \vec{E} \times \vec{B}}=\frac{\partial}{\partial \hat{r}}\left(m_{i} n_{i} v_{\|, i} \frac{\partial \phi}{\partial \hat{\theta}}\right)-\frac{\partial}{\partial \hat{s}_{\|}}\left(\frac{|B| B_{\phi}}{B_{\theta}} m_{i} n_{i} v_{\|, i} \frac{\partial \phi}{\partial \hat{r}}\right)
$$

The $\vec{E} \times \vec{B}$ particle and momentum sources were integrated into the OSM numerical scheme by first calculating a plasma solution without the drift terms, calculating the $\vec{E} \times \vec{B}$ sources and calling the numerical solver once more. This process is iterated with the EIRENE code until convergence is reached. In order to improve numerical stability, the $\vec{E} \times \vec{B}$ sources in OSM-EIRENE are multiplied by a factor ranging between 0.01 and 1 , which increases with each successive iteration. The code is then iterated with 
the factor set to 1 to ensure full convergence.

The source terms were calculated in a finite-volume manner, by linearly interpolating drift fluxes to cell edges both parallel and orthogonal to field lines and dividing the difference between the incoming and outgoing drift fluxes by the parallel and cross-field cell widths respectively. The calculation of the radial and poloidal $\vec{E} \times \vec{B}$ drift velocities was checked against a simple analytic estimate of these quantities [8]:

$$
\begin{gathered}
v_{\vec{E} \times \vec{B}}^{p o l} \approx \frac{3 T_{e}}{\lambda_{T_{e}} B} \\
v_{\vec{E} \times \vec{B}}^{r a d} \approx \frac{B}{B_{\theta}} \frac{T_{e}}{2 L_{\|} B}
\end{gathered}
$$

where $\lambda_{T_{e}}$ is the radial temperature fall-off length $(\mathrm{m})$ and $L_{\|}$is the parallel connection length $(\mathrm{m})$, all other terms have their usual meanings. In the analytic calculation of the poloidal $\vec{E} \times \vec{B}$ drift velocity, expression in (4.42) was modified to take into account the poloidal variation in the temperature fall-off length. The modified equation is:

$$
v_{\vec{E} \times \vec{B}}^{p o l}=\frac{3 T_{e}}{\lambda_{T_{e}} B} \frac{\left(B / B_{\theta}\right)}{\left(B / B_{\theta}\right)_{u}}
$$

where $\left(B / B_{\theta}\right)_{u}$ and $\left(B / B_{\theta}\right)$ are the poloidal flux expansion, the ratio of the total to the poloidal magnetic field strengths at the upstream location (taken to be the outboard midplane) and at a given location, respectively. In this calculation, $\lambda_{T_{e}}$ is taken to be $2.5 \mathrm{~cm}$, which is indicative of radial temperature fall-off lengths observed on MAST [38]. With this correction term in place, the OSM and analytically calculated poloidal and radial drift velocities agree quite well (figure 4.16). Previous studies [79,84] have shown that the $\vec{E} \times \vec{B}$ drift velocity modifies the ion velocity (Bohm-Chodura) at the entrance to the magnetic pre-sheath, requiring the total projected poloidal (i.e. parallel) velocity be equal to the sound speed, $c_{s}$. In the implementation of a drift model presented here, the above condition is automatically satisfied, as the primary velocity in the code is the parallel velocity, and anomalous particle and momentum sources can be calculated such that $v_{i}= \pm c_{s}$ at the edge of the grid close to material surfaces.

Analysis of the effects of the $\vec{E} \times \vec{B}$ drift is complicated by experimental uncertainties in the data used to constrain the OSM-EIRENE code. In order to assess their impact on converged calculations, the code was run several times, shifting the ion flux specified at the lower outer divertor plate (figure 4.17) as specified by Langmuir probe data. The radial $\vec{E} \times \vec{B}$ drift velocity on the separatrix ring was compared when the code had converged in each case, as the separatrix is most affected by such changes. The analysis shows that modest shifts in the experimental data can result in wide scatter in the converged drift velocities. For flux tubes in close proximity to the separatrix, the 


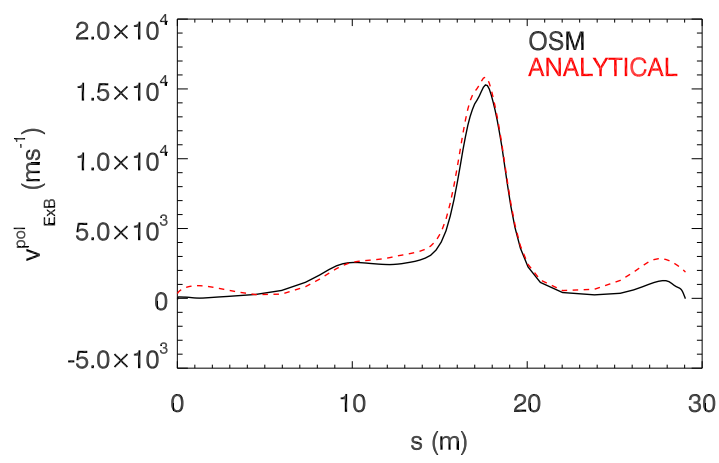

(a) Comparison of OSM calculated (black) and an approximate calculation (red) of the poloidal $\vec{E} \times \vec{B}$ drift velocities.

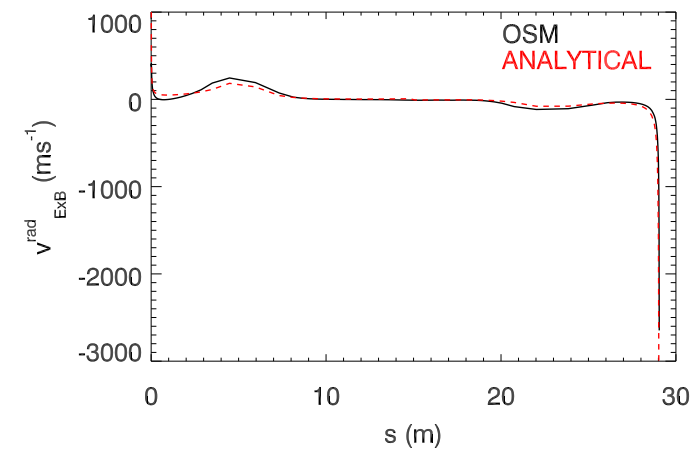

(b) Comparison of OSM calculated (black) and approximate calculation (red) of thr radial $\vec{E} \times \vec{B}$ drift velocities.

Figure 4.16: Comparison of analytical estimates and OSM calculations of $\vec{E} \times \vec{B} \mathrm{drift}$ velocities.

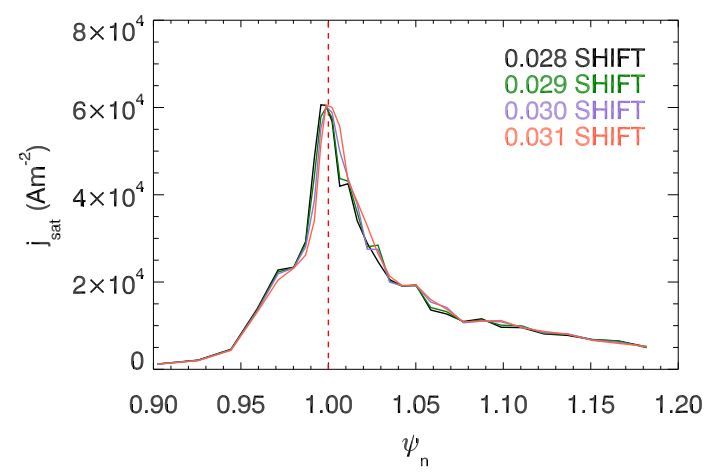

(a) Shifted ion saturation current profiles specified at the lower outer strike point.

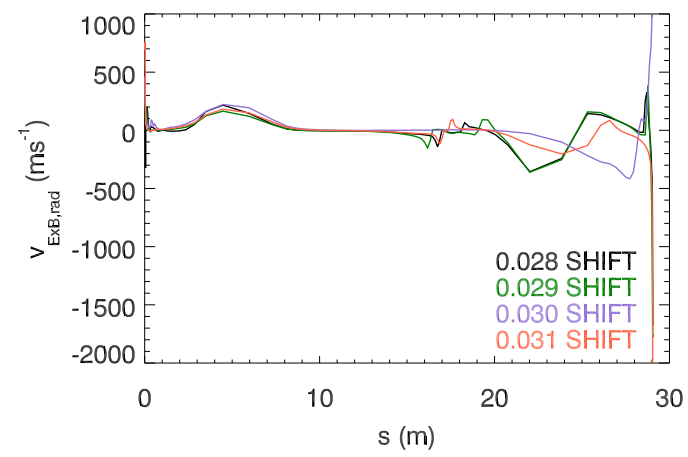

(b) Radial $\vec{E} \times \vec{B}$ profile along the separatrix ring calculated by OSM-EIRENE for different shifts in the lower outer ion saturation current data.

Figure 4.17: Sensitivity analysis of radial $\vec{E} \times \vec{B}$ velocities to shifts in ion saturation current profiles at the lower outer target.

scatter in the converged solution with shifting the Langmuir probe data is reduced. As a result, this study will focus on the effects of the $\vec{E} \times \vec{B}$ drift on a flux tube adjacent to the separatrix ring in the outer scrape-off layer displaced approximately $1 \mathrm{~mm}$ radially from the separatrix ring, as measured at the outboard midplane.

In the vicinity of the inner strike point, the $\vec{E} \times \vec{B}$ drift results in an increase in particle density as the radial drift transports particles from the near-SOL into the far SOL (figure 4.18). Conversely, particle density close to the outer strike point decreases, as the radial drift motion transports particles from the far-SOL to the near-SOL. Such behaviour is consistent with experimental observations from reversed field experiments [79]. The effects on the ion flow profiles is more complex, as the mostly positive poloidal $\vec{E} \times \vec{B}$ velocity competes with parallel flow driven by EIRENE particle ionisation sources 


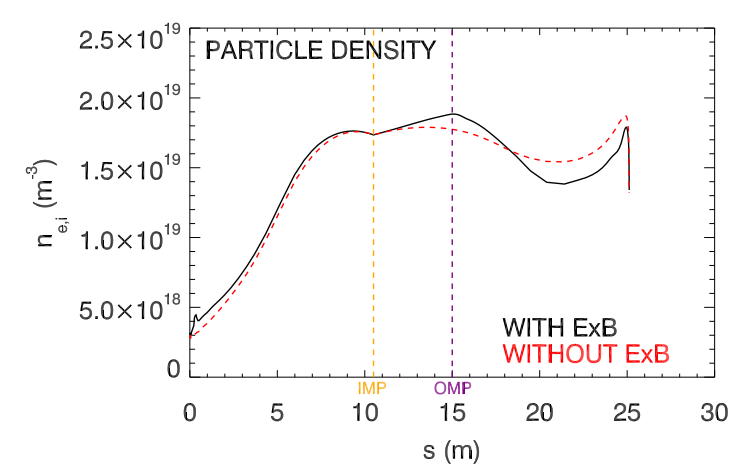

(a) Particle density profile in the near-SOL with and without $\vec{E} \times \vec{B}$ drift terms.

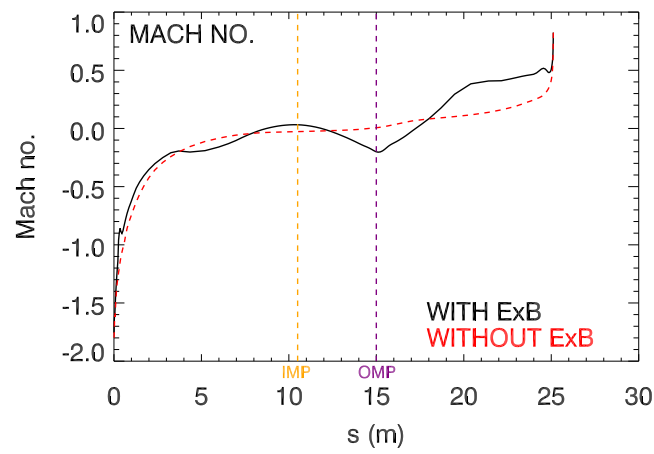

(b) Ion velocity profile in the near-SOL with and without $\vec{E} \times \vec{B}$ drift terms.

Figure 4.18: Near-SOL density and parallel Mach number profiles with and without the effects of $\vec{E} \times \vec{B}$ drift motion.

and additional particle sources generated by radial drift motion. In the presence of drifts, figure 4.18, the ion flow profile has characteristics in common with the poloidal flow profiles observed in other machines [74]. For example, the ion flow is directed toward the inner divertor (negative) across most of the SOL, except in the vicinity of the outer divertor target. A strong peaking in the parallel velocity is observed at the outer midplane, where radial gradients in $n_{e}, T_{e}$ are greatest, and hence a strong radial electric field exists. This in turn leads to strong particle and momentum source generation due to the rapid spatial variation in the parallel $\vec{E} \times \vec{B}$ drift flux (figure 4.20). Contrary to OSM solver behaviour when other terms are introduced into the conservation equations, the introduction of sources due to $\vec{E} \times \vec{B}$ drift motion caused an increase in the solver fitting source, required to ensure the plasma solution is in agreement with experimental data, by approximately a factor of five. A possible explanation for this behaviour is that in the fluid model of plasma transport, the $\vec{E} \times \vec{B}$ drift is seldom found in isolation of other drifts, such as the diamagnetic drift. As a result, it could be that additional drift terms, combined with $\vec{E} \times \vec{B}$, are required to ease solver convergence towards measured values.

In terms of explaining the pressure drop between the outboard midplane and the lower outer divertor target, the $\vec{E} \times \vec{B}$ drift has quite a weak influence on the pressure profile overall, except in the vicinity of the outer midplane (figure 4.19), where the total pressure is elevated. The stronger drift effects on field lines closer to the separatrix may result in a more pronounced influence on the pressure profile, which may go some way towards explaining the pressure drop. On the flux tube under consideration, however, the $\vec{E} \times \vec{B}$ drift cannot explain the observed pressure decrease from the outboard midplane to the lower outer target. 


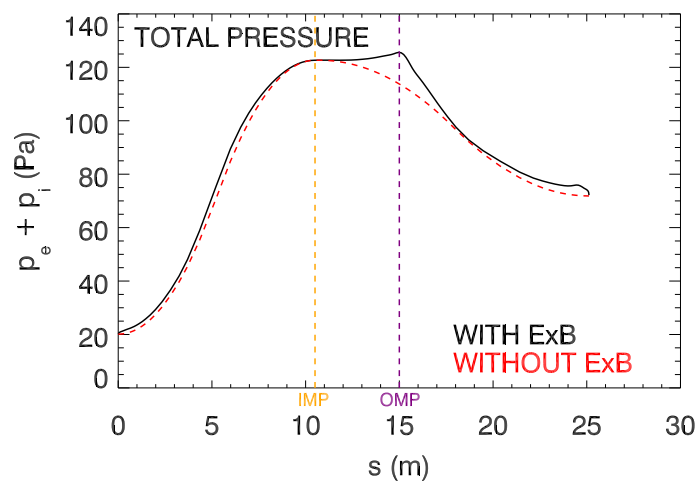

Figure 4.19: Variation in total (ion+electron) plasma pressure along a field line close to the magnetic separatrix, both with and without $\vec{E} \times \vec{B}$ terms included in the calculation.

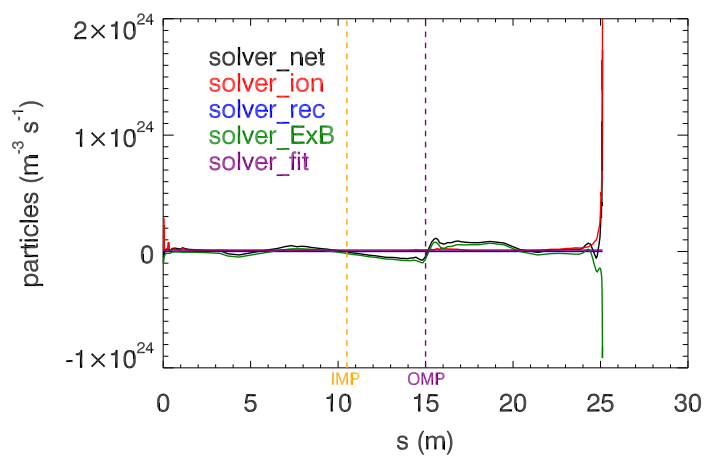

(a) Distribution of particle sources in the near SOL with the inclusion of ExB drift sources.

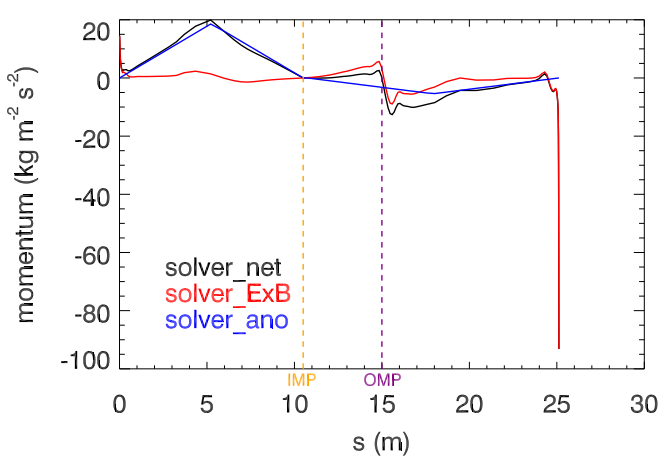

(b) Distribution of momentum sources in the near SOL with the inclusion of ExB drift sources

Figure 4.20: Particle and momentum sources in the near SOL in MAST, including ExB drift motion. 


\subsubsection{The Diamagnetic Drift}

In a similar manner to section 4.5.1, the particle source due to the electron diamagnetic drift can be derived. Starting with the expression:

$$
S_{p, d i a}=-\vec{\nabla} \cdot\left(n_{e} v_{e, d i a}\right)=-\vec{\nabla} \cdot\left(\frac{\vec{B} \times \vec{\nabla} p_{e}}{-e B^{2}}\right)
$$

which can be expanded to give:

$$
\begin{aligned}
S_{p, d i a} & =\frac{\partial}{\partial \hat{r}}\left(-\frac{B_{\phi}}{e B^{2}} \frac{\partial p_{e}}{\partial \hat{\theta}}\right)+\frac{\partial}{\partial \hat{\theta}}\left(\frac{B_{\phi}}{e B^{2}} \frac{\partial p_{e}}{\partial \hat{r}}\right) \\
& =\frac{\partial}{\partial \hat{r}}\left(-\frac{B_{\phi}}{e B^{2}} \frac{\partial p_{e}}{\partial \hat{\theta}}\right)+\frac{\partial}{\partial \hat{s}_{\|}}\left(\frac{B_{\phi}}{e B_{\theta}|B|} \frac{\partial p_{e}}{\partial \hat{r}}\right)
\end{aligned}
$$

In this model, it is assumed that the diamagnetic (or Pfirsch-Schlüter) current is the sole means of driving cross-field current, an additional source arises due to the $-\vec{\nabla} \cdot\left(\frac{j_{\|} \hat{b}}{e}\right)$ term in (4.33). Therefore, using the current conservation equation:

$$
\nabla_{\|}\left(j_{\|} \hat{b}\right)+\nabla_{\perp} j_{\perp}=0
$$

where $j_{\|}$and $j_{\perp}$ are the parallel and cross-field current densities respectively, this can be expressed in the form:

$$
\nabla_{\|}\left(\frac{j_{\|} \hat{b}}{e}\right)+\vec{\nabla} \cdot\left(n_{i} v_{i, d i a}-n_{e} v_{e, d i a}\right)=0
$$

where $v_{i, d i a}$ and $v_{e, d i a}$ are the ion and electron diamagnetic velocities respectively. This can be expressed in the form

$$
\nabla_{\|}\left(\frac{j_{\|} \hat{b}}{e}\right)=-\vec{\nabla} \cdot\left(\frac{\vec{B} \times \vec{\nabla} P}{B^{2}}\right)
$$

where $P=p_{e}+p_{i}$. This can be expanded in a manner similar to (4.46) to facilitate Combining (4.45) and (4.49) yields the electron conservation equation with diamagnetic drift terms:

$$
\vec{\nabla} \cdot\left(n_{e} v_{\|, i} \hat{b}\right)=S_{p}-\vec{\nabla} \cdot\left(\frac{\vec{B} \times \vec{\nabla} p_{e}}{-e B^{2}}\right)+\vec{\nabla} \cdot\left(\frac{\vec{B} \times \vec{\nabla} P}{B^{2}}\right)
$$

A diamagnetic drift term is not present in the parallel momentum equation as a cancellation occurs between this term and a viscosity term arising from Lamor rotation (gyro rotation), known as the diamagnetic cancellation $[72,85]$. Unlike the 
$\vec{E} \times \vec{B}$ drift, the diamagnetic drift is known to be mostly divergence free, meaning that the overall drift motion should not significantly alter the fluxes to plasma-facing components. However, the divergent part of the diamagnetic drift is proportional to $\frac{\nabla_{\|} B}{B}$, meaning that a larger diamagnetic drift flux would be expected to reach plasmafacing components in a spherical Tokamak geometry. In the OSM model used here, the target flux will be unaffected by the presence of drift sources, as the numerical scheme is designed to constrain these fluxes to take prescribed values from experimental data.

The OSM-EIRENE code was run with the calculated particle sources in (4.50) and iterated until convergence was reached, analogous to the implementation of the $\vec{E} \times \vec{B}$ drift. The diamagnetic drift sources, however, are less sensitive to small mis-alignments between the magnetic grid and the Langmuir probe data at the lower outer target plate. As a result, the separatrix ring is analysed in this section. For the purposes of comparison, OSM-EIRENE was run with the same input data but with the diamagnetic source terms turned off in order to ascertain their effects. The density profile along the separatrix ring (figure 4.21) shows that the density has been re-distributed from the inboard to the outboard side, where the diamagnetic source terms are stronger. This is due to stronger radial pressure gradients existing on the outboard side, which is a consequence of the Shafranov shift and the "ballooning" nature of cross-field transport, being stronger on the low-field side. As expected, the integral of the particle source is small ( $<7 \%$ of the total particle source), reflecting that the diamagnetic drift is mostly divergence-free, even in a spherical Tokamak geometry. In addition, the sign of the diamagnetic particle source is positive on the low-field side, suggesting that particles are entering the plasma core via cross-field transport from the scrape-off layer. The reverse occurs at the low-field side midplane (figure 4.24). This is in agreement with the basic characteristics of the diamagnetic drift [8].

In terms of addressing the parallel pressure imbalance observed in MAST discharges, the addition of the diamagnetic drift terms served to raise the total pressure in the vicinity of the outboard strike point (at large $s$ ). As a result, the pressure discrepancy between the outboard midplane and the outer divertor target has reduced. Furthermore, the introduction of diamagnetic drift terms, resulted in a reduction in the anomalous particle source term by approximately $20 \%$. The electrical current arising from the diamagnetic drift will also influence the calculated parallel potential profiles via the $\eta_{\|} j_{\|}$term in (4.24). When the presence of the diamagnetic current source $S_{j}$ is included in (4.26), which manifests as a $-\eta S_{j}$ term on the right-hand side, the resulting potential profile is only slightly affected (figure 4.23 ). This is because the contribution of electrical currents in (4.24) is weighted by the plasma electrical resistivity, $\eta_{\|}$, which is normally small, approximately $3 \times 10^{-5} \mathrm{ohm} . \mathrm{m}$ when $T_{e}=10 \mathrm{eV}$.

Accurate calculation of drift behaviour close to divertor plates in MAST is difficult 


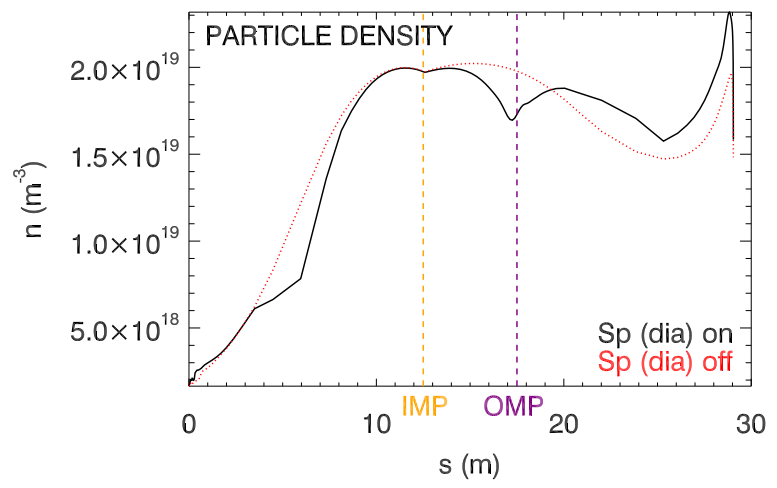

Figure 4.21: Electron (or ion) density profile along the separatrix ring for MAST shot 24082 at $240 \mathrm{~ms}$. The density profile when the diamagnetic source terms are turned on (black) is significantly different to the density profile with no drift terms turned on (red).

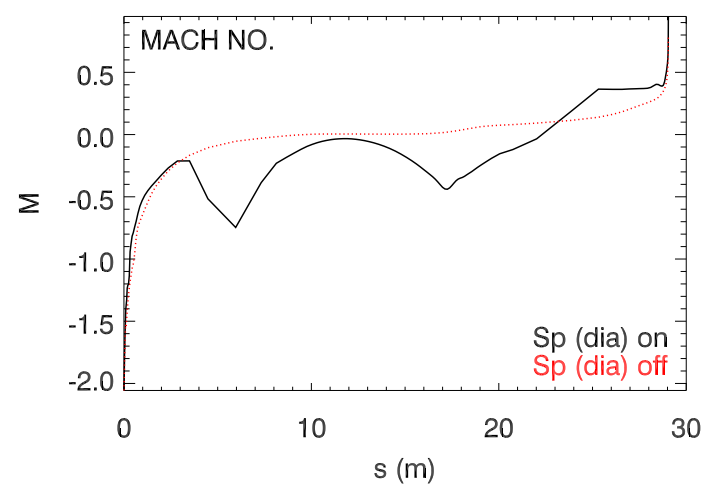

(a) Ion mach number profile along the separatrix ring, with and without diamagnetic drift terms. The velocity profile is strongly influenced by the presence of the separatrix, in the vicinity of which the flow accelerates.

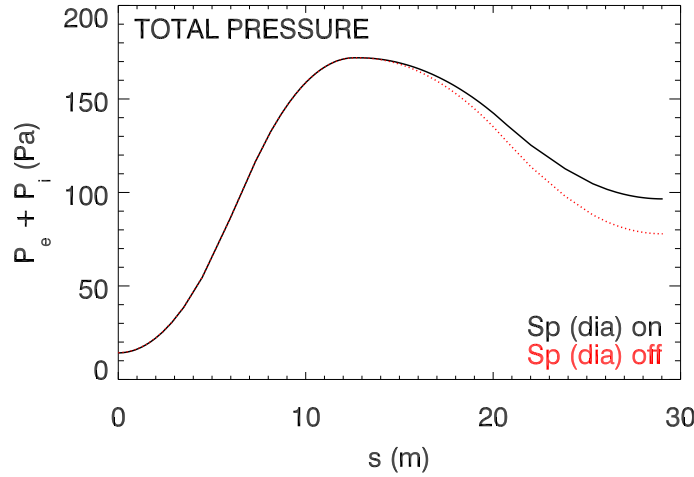

(b) Total pressure profile along the separatrix ring in OSM-EIRENE runs with (black) and without (red) diamagnetic drift terms turned on.

Figure 4.22: The effects of the diamagnetic drift on parallel Mach number and pressure profiles along the separatrix ring. 


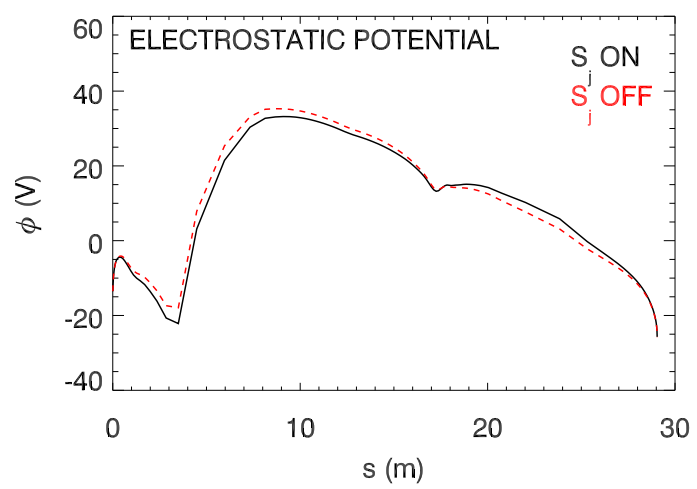

Figure 4.23: Parallel electrostatic potential profiles along the separatrix ring with (black) and without (red) diamagnetic current sources included in the calculation. Diamagnetic particle sources were included in both calculations.

due to the high poloidal flux expansion at the outer target locations (normally between 5 and 6) decreasing radial grid resolution. Furthermore, close proximity to where the grid terminates reduces scope to interpolate plasma conditions to calculate accurate gradients. As a result, the numerical algorithm uses a first-order approximation of the derivative operator at cells adjacent to divertor surfaces, thereby reducing the accuracy of the cross-field drift source calculation. Following an analysis by Chankin [86], no modifications of the boundary conditions specified by experimental data are carried out to accommodate the effects of the diamagnetic drift.

\section{6 $\mathbf{T}_{i}=\mathbf{T}_{e}$ Assumption}

Energy-exchange collisions that take place between ions and electrons have the effect of heating ions and cooling electrons. Consequently, ion temperature measurements in diverted Tokamaks report $T_{i}>T_{e}[87,88]$. These processes are represented in the ion and electron energy equations as a source and sink of energy respectively [8]:

$$
Q_{e q}=\frac{3 m_{e}}{m_{i}} n_{i} \nu_{e q} e\left(T_{e}-T_{i}\right)
$$

where:

$$
\nu_{e q} \approx 2.9 \times 10^{-12} n_{i} \ln \Lambda T_{e}^{3 / 2}
$$

is the ion-electron energy exchange collision frequency $\left(s^{-1}\right), \ln \Lambda$ is the Coulomb $\operatorname{logarithm}(\approx 17)$. McCracken [89], showed that for electrons and ions to be in thermal equilibrium, assuming that the electrostatic sheath is the principal cooling mechanism for electrons, the following criterion must be satisfied: 


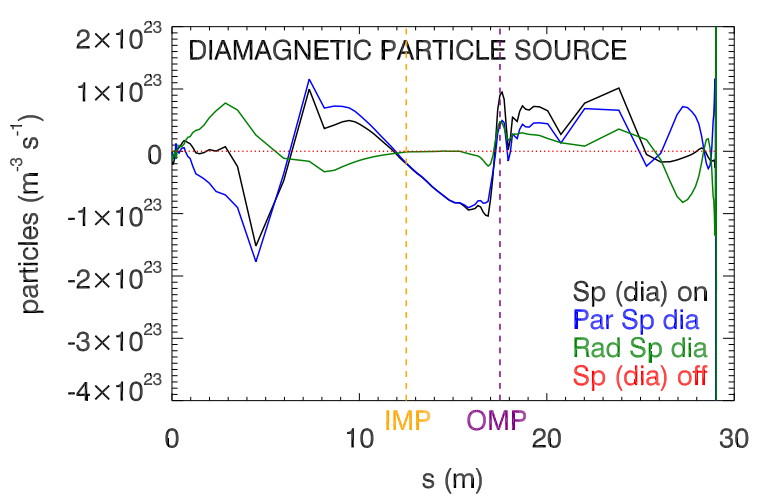

Figure 4.24: Distribution of the diamagnetic particle source, and it's parallel and radial components, along the separatrix ring of MAST shot 24861 at $240 \mathrm{~ms}$. The diamagnetic drift acts as a particle source at the high-field midplane and a sink at the low-field side as particles are transported from the main plasma into the SOL in the former case and from the SOL into the main plasma in the latter case.

$$
\frac{L_{\|} n_{e}}{T_{e}^{2}} \geq 1.0 \times 10^{17}
$$

where $L_{\|}, T_{e}$ and $n_{e}$ have their usual meanings, and the constant on the RHS has units of $\left(\mathrm{m}^{-2} \mathrm{eV}^{-2}\right)$. In plasma conditions taken from MAST discharge 24861, $T_{e} \approx 25 \mathrm{eV}, n_{e} \approx 2.0 \times 10^{19} \mathrm{~m}^{-3}, L_{c} \approx 15 \mathrm{~m}$, which indeed satisfies the criterion in (4.53). More detailed modelling of lower density MAST discharges [73], indicated values of $T_{i} / T_{e}>2$, perhaps suggesting that the assumptions made in the previous analysis are not entirely valid on MAST due to the presence of other energy loss mechanisms taking place in the MAST SOL, such as ionisation and charge-exchange interactions with main chamber neutrals.

In order to more accurately assess the implications of $T_{i} \neq T_{e}$ on the OSM plasma solution, a number of OSM-EIRENE runs were made with varying (specified) values of $T_{i} / T_{e}$, which was held constant along field lines. This behaviour is consistent with prior OSM2 modelling [73], where electron and ion energy conservation equations were solved to calculate $T_{i}$ and $T_{e}$. Figure 4.25 shows the density and pressure profiles on the separatrix ring for geometry and experimental data taken from MAST discharge 24861 at $240 \mathrm{~ms}$. $\mathrm{T}_{i} / \mathrm{T}_{e}$ is increased from 1 to 3 , covering the range of expected values from previous modelling of heat transport in MAST scrape-off layer [73]. In terms of explaining the pressure imbalance between the upstream and target plasma conditions, increasing $\mathrm{T}_{i} / \mathrm{T}_{e}$ increases this imbalance, as the target ion flux is constrained by Langmuir probe data at the divertor targets, so increasing $\mathrm{T}_{i}$ increases the target ion sound speed, and consequently reduces the particle density in the vicinity of the target 


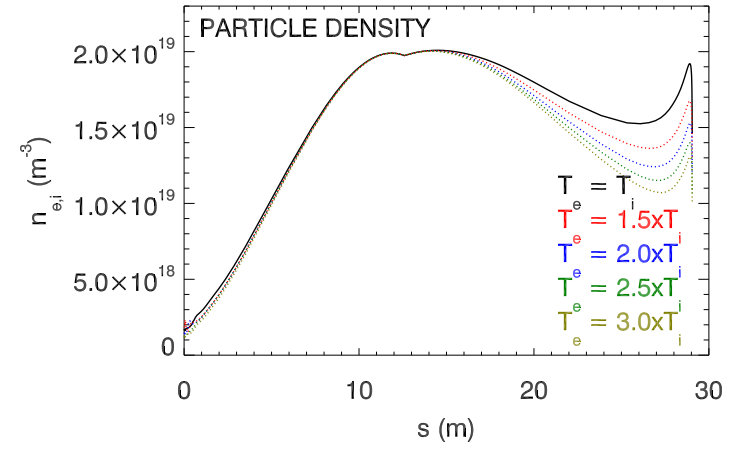

(a) Density profiles close to the magnetic separatrix in MAST shot 24861 assuming different values for $\mathrm{T}_{i} / \mathrm{T}_{e}$.

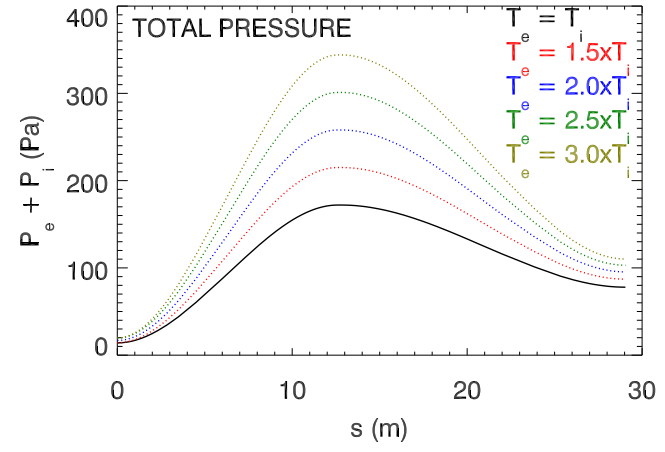

(b) Total (ion+electron) pressure profiles close to the magnetic separatrix in MAST shot 24861 , assuming different values for $\mathrm{T}_{i} / \mathrm{T}_{e}$.

Figure 4.25: OSM-EIRENE simulations of the effects of varying $\mathrm{T}_{i} / \mathrm{T}_{e}(\gamma)$.

plates. The electron density and temperature are specified at the inboard midplane by Thomson scattering measurements, hence the identical values of particle density at that location. Poloidal variations in $\mathrm{T}_{i} / \mathrm{T}_{e}$, which are not accounted for in this simple analysis, would alter the pressure balance along field lines and may help to explain the apparent pressure drop observed here, for instance, if $\mathrm{T}_{i} / \mathrm{T}_{e}$ were 2 and 3 at the outer midplane and lower outer target plate respectively. However, knowledge of the poloidal variations in $\mathrm{T}_{i} / \mathrm{T}_{e}$ would require measurements of both quantities at multiple locations, which is not currently possible on MAST. It has also been noted that varying $\mathrm{T}_{i} / \mathrm{T}_{e}$ from 1 to 3 reduces the particle fitting source by factors of 1 and 1.5 respectively.

\subsection{Summary}

The new numerical schemes developed for use with OSM have been used to incorporate a more advanced plasma transport model into the code. These were used to assess the impact of different transport terms on density and parallel ion velocity profiles and to find an explanation for an anomalous pressure drop that occurs between the outboard midplane and the lower outer strike point, even in attached conditions.

It has been found that the thermoelectric current is largely responsible for the plasma current detected at the divertor target plates, which has been observed on large aspect ratio Tokamaks, but not in STs. Study of transport terms arising from flux expansion found that they had a significant impact on the numerical solver's convergence properties, requiring fitting sources almost an order of magnitude smaller to allow the plasma solution to coincide with experimental measurements. The flux expansion terms were also found to alter the parallel potential profile, which formed a peak near the inner midplane as a result of $\nabla_{\|} B / B$ being high in this region. However, the flux expansion terms could not explain the pressure drop between the 
outer midplane and target.

Parallel viscosity was found to have the effect of smoothing the parallel ion velocity profile and consequently reducing the plasma density in the vicinity of the divertor target plate. This resulted in an increased pressure drop in the vicinity of the lower outer target plate, but not of sufficient magnitude to explain experimental observations. The viscosity transport terms did, however, assist in solver convergence, by reducing the solver fitting particle source by approximately a factor of 2 .

Poloidal and cross-field drift motion was incorporated into the OSM numerical scheme by representing the effects of drifts as additional source terms. Iteration between the numerical solver and the drift source calculation was required in order to reach convergence. It was found that the $\vec{E} \times \vec{B}$ drift sources are very sensitive to small shifts in ion saturation current profiles specified at the divertor targets using Langmuir probe data, so an analysis was carried out on an adjacent flux tube in the near-SOL. The calculated drift velocities were in good agreement with simple analytical models and that the additional sources led to an increase in plasma pressure at the outboard midplane and the vicinity of the lower outer divertor target. However, the $\vec{E} \times \vec{B}$ drift terms also elevated the anomolous particle fitting source term by approximately a factor of 5 .

The diamagnetic drift source calculation appeared to be slightly more robust with regard to small displacements in the Langmuir probe data at the target plate. The diamagnetic drift source was found to be mostly divergence free, in agreement with basic properties of diamagnetic drift motion. These terms also brought about a significant decrease in the pressure differential between the outboard midplane and the lower outer divertor target, whilst reducing the anomalous particle fitting source by $20 \%$. It was also observed that Pfirsch-Schlüter currents had a modest effect on the potential profile calculation. Finally, it has been found that uncertainties in the ion temperature at the midplane and divertor targets are unlikely to explain the observed pressure drop. The effects of the additional phyiscal mechanisms on solver performance and in explaining the pressure discrepancy between the outboard midplane and the lower outer divertor target are summarised below:

\begin{tabular}{|c|c|c|}
\hline Physical Mechanism & Solver Convergence & Pressure Discrepancy \\
\hline Flux expansion & Improved $(\times 5)$ & Small effect \\
\hline Parallel viscosity & Improved $(\times 2)$ & Increased \\
\hline$\vec{E} \times \vec{B}$ drift & Negative $(\times 5)$ & Increased \\
\hline Diamagnetic drift & Improved $(\times 1.2)$ & Reduction \\
\hline $\mathrm{T}_{i} \neq \mathrm{T}_{e}$ & Improved $(\times 1 \rightarrow \times 1.5)$ & Unknown \\
\hline
\end{tabular}




\section{Chapter 5}

\section{Detachment on MAST}

In this chapter, experimental data of detached divertor conditions on MAST is presented from Thomson scattering, Langmuir probes and divertor imaging and line-of-sight spectroscopy. The analysis and interpretation of the data is discussed, in particular how uncertainties arise and how they influence conclusions that can be drawn from the data. The information collected is input into the interpretive OSM-EIRENE code to investigate consistency between the data collected from different diagnostics and attempt to clarify the physical mechanisms that give rise to divertor detachment on MAST. The quality of the OSM-EIRENE plasma solution is verified using comparisons between simulated and experimental observations of the strength of $D_{\gamma} / D_{\alpha}$ emission line ratios.

\subsection{Introduction}

Initial studies into divertor detachment on MAST were carried out by Tabasso [90] in L-mode discharges with $600 \mathrm{kA}$ plasma current and $750 \mathrm{~kW}$ of auxiliary heating provided by a neutral beam injector. In these experiments, the core (line averaged) density was increased from $3 \times 10^{19} \mathrm{~m}^{-3}$ to $7.0 \times 10^{19} \mathrm{~m}^{-3}$ by increasing the gas fuelling rate from piezo valves located at the midplane and upper and lower divertor regions. This study was carried out when the MAST original divertor was in place (figure 5.1), which consisted of 12 radial ribs.

Using the target Langmuir probes in swept mode the ion saturation current, electron density and temperature were measured at the target plates and spectrometers were used to monitor $\mathrm{D}_{\alpha}$ and $\mathrm{D}_{\gamma}$ emission from the outer strike point. The outcome of the study was an indication that detachment was occurring at all divertor strike points as the density was ramped, with degrees of detachment of 22 and 6 at the inner and outer strike points respectively. Analysis of the ratio of $\mathrm{D}_{\gamma}$ and $\mathrm{D}_{\alpha}$ emission at the outer target indicated a decrease as the core density was increased, followed by an increase as the plasma made the transition to detachment. This observation is contrary to observations 


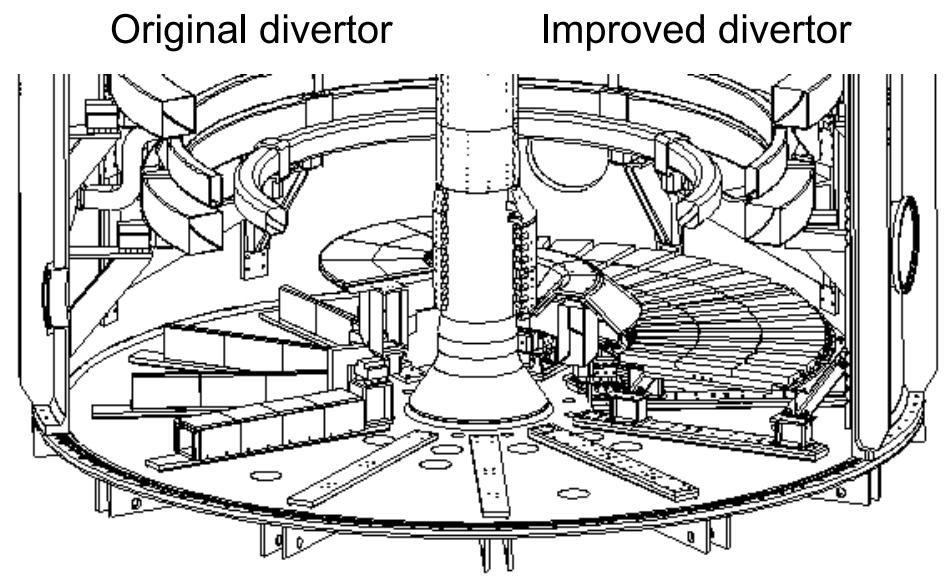

Figure 5.1: The original MAST divertor (used prior to 2004) and the current improved divertor. [37]

on other devices such as JET [91], ASDEX-Upgrade [92] and Alcator-Cmod [93] and no explanation was given for this behaviour. Furthermore, as the divertor diagnostics available at the time were limited, the quantity of data collected was insufficient to make conclusions on the physical mechanisms that gave rise to detachment in this case.

In this chapter, analysis of data from a wide array of high-quality diagnostic data is presented from shot 25028 at $312 \mathrm{~ms}$, with an emphasis placed upon examining the plasma conditions within the detached lower inner divertor leg. The data is examined for self-consistency and how the data is interpreted in terms of consistency with observations of detached plasmas made on other devices. This study is complementary to those carried out on the NSTX spherical tokamak [94-96], where the operating space over which detachment of the inner divertor leg has been extensively examined.

\section{$5.2 \quad$ Two-Point Modelling}

The two-point model, explained in chapter 2, can be used as a tool to predict the upstream plasma conditions that could give rise to detachment at the target. However, the basic two-point model loses validity in detached conditions, as presumption pressure conservation, to name one example, is no longer valid. Therefore, a number of corrections are required before the two-point model can be used for this purpose.

To account for momentum loss processes, taken to be charge-exchange and radiative recombination, a momentum loss term $f_{m}$ is included in the momentum conservation equation [11]

$$
2 n_{t} T_{t}=f_{m} n_{u} T_{u}
$$


where $n_{t}, T_{t}, n_{u}, T_{u}$ are the plasma density and temperature at the target and upstream locations respectively. The form of $f_{m}$ is given by:

$$
\begin{gathered}
f_{m}=2\left(\frac{\alpha}{\alpha+1}\right)^{\frac{\alpha+1}{2}} \\
\alpha=\frac{\langle\sigma v\rangle_{i}}{\langle\sigma v\rangle_{i}+\langle\sigma v\rangle_{m}}
\end{gathered}
$$

where $\langle\sigma v\rangle_{i}$ is the rate coefficient for ionisation and $\langle\sigma v\rangle_{m}$ is the rate coefficient for momentum loss processes. The form of $f_{m}$ used here is based on work by Self and Ewald [97], where analytic solutions for the electron density an isothermal 1D plasma with ionisation and friction procceses at the entrance to a recycling region. Using atomic data from ADAS [98], the magnitude of $f_{m}$ can be calculated:
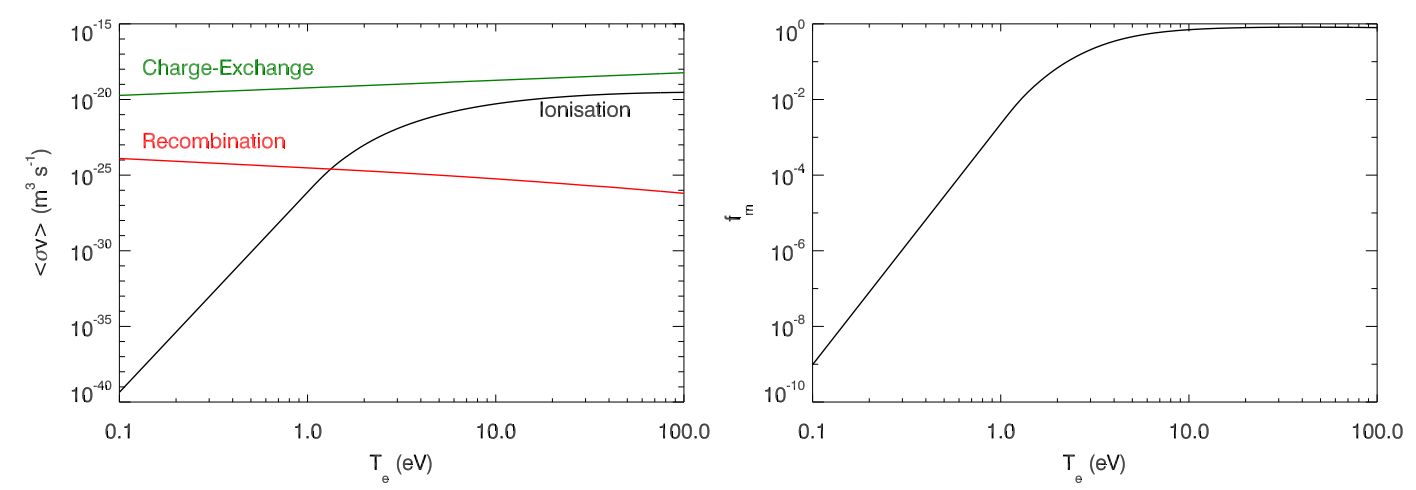

Figure 5.2: Ionisation, charge-exchange and radiative recombination rate coefficients from ADAS for the calculation of the momentum-loss term $f_{m}$

At typical attached target conditions, $T_{e}>10 \mathrm{eV}, f_{m}$ is small, as would be expected. However, when $T_{e}<10 \mathrm{eV}, f_{m}$ becomes increasingly smaller, indicating that momentum-loss processes become more frequent, and hence have a stronger effect on target conditions, as the target temperature drops. The effects of molecular processes are not included in this model, which reduces $f_{m}$ below $T_{e}=10 \mathrm{eV}$, however, it has been noted [11], that the results of the two point model are similar irrespective of whether molecular effects are included in the calculation of $f_{m}$.

Refinements of the power balance equation can be made by including the effects of hydrogen line radiation emitted before a recycled atom is ionised. This term is given by:

$$
q_{\text {Hrad }}=e n_{t} c_{s, t} \epsilon_{\text {rad }}
$$

where $c_{s, t}$ is the ion acoustic velocity and $\epsilon_{\text {rad }}$ is given by an empirical approximation [99]: 


$$
\epsilon_{\text {rad }}=17.5+\left[5+\frac{37.5}{T_{e}}\left(1+\frac{0.2533}{T_{e}}\right)\right] \log \left(\frac{10^{21}}{n_{e}}\right)
$$

This formula is valid when $n_{e}<10^{21} \mathrm{~m}^{-3}$ and $T_{e}>17.5 \mathrm{eV}$. In this study, the approximation of $\epsilon_{r a d}$ is used in conditions that satisfy the condition on $n_{e}$ but not $T_{e}$ as the solution approaches detached conditions. Therefore, the use of this approximation is an additional source of error in the predictions made using this model. An additional source in the power balance equation arises from the ionisation potential energy (13.6eV in the case of hydrogen) and half of the binding energy of a hydrogen molecule $(\approx 2.2 \mathrm{eV})$ that is deposited on the divertor target plate as heat when incident ions strike the target, recombine to form atoms, which form molecules on the surface [11]. The magnitude of this term is given by:

$$
q_{p o t}=e n_{e} c_{s, t} \epsilon_{p o t}
$$

where $\epsilon_{\text {pot }}$ is approximately $15.8 \mathrm{eV}$, which is a constant. Finally, the effects of impurities emitting line radiation over the connection length $L_{\|}$can also be approximated and incorporated into this model. Le [100] calculated a term in the two-point model to account for line radiation due to carbon impurities, which will be adopted here. It results in a modified form of the power balance equation:

$$
q_{t}=q_{u}\left(1-f_{r a d, c}\right)=e n_{t} c_{s, t}\left(\gamma T_{t}+\epsilon_{r a d}+\epsilon_{p o t}\right)
$$

where

$$
f_{r a d, c}=1-\sqrt{1-\frac{14}{3} \frac{c_{c} L_{z} n_{u}^{2} L_{\|}}{q_{u}}}
$$

$c_{c}$ is the concentration of carbon atoms and $L_{z}$ is the radiation constant, which can be approximated as [101]:

$$
\begin{gathered}
L_{z}=1 \times 10^{-31}\left(T_{e}>8 \mathrm{eV}\right) \\
L_{z}=7 \times 10^{-35} T_{e}^{\frac{7}{2}}\left(1<T_{e}<8 \mathrm{eV}\right)
\end{gathered}
$$

These corrections result in the following set of equations:

$$
\begin{gathered}
T_{u}^{\frac{7}{2}}=T_{t}^{\frac{7}{2}}+\frac{7 q_{u} L_{\|}}{2 \kappa_{0}} \\
2 n_{t} T_{t}=f_{m} n_{u} T_{u}
\end{gathered}
$$




$$
q_{u}\left(1-f_{\text {rad,c }}\right)=e n_{t} c_{s, t}\left(\gamma T_{t}+\epsilon_{\text {pot }}+\epsilon_{\text {rad }}\right)
$$

Unlike the basic two-point model, the additional corrections introduced here necessitate the use of a numerical solver to calculate $T_{u}, T_{t}, n_{t}$ for a given $q_{u}, n_{u}$, as $f_{m}$ has no analytical form. However, the inclusion of these additional terms capture some of the processes that play a role in the conduction-limited and detached divertor regimes. In this study, a Newton-Raphson method is used to solve the above equations, where a finite difference approximation of the Jacobian is computed according to [67]. The upstream power flux $q_{u}$ is calculated according to [11]:

$$
q_{u}=\frac{1}{4 \pi a \sqrt{\kappa} N} \frac{P_{S O L} q_{95}}{\lambda_{p}}
$$

where $a$ is the horizontal plasma minor radius, $\kappa$ is the elongation (the ratio of the vertical and horizontal minor radii), $N$ is the number of poloidal field nulls, $q_{95}$ is the safety factor at normalised magnetic flux $=0.95, P_{S O L}$ is the power crossing the magnetic separatrix (W) and $\lambda_{p}$ is the 1 /e falloff length for power incident on the divertor strike point $(\mathrm{m})$.

The detachment threshold was estimated in a lower single-null plasma where $q_{u} \approx$ $4.1 \times 10^{6} \mathrm{Wm}^{-2}, \mathrm{q}_{95}=2.8, \mathrm{a}=2 \mathrm{~m}, \kappa=1.8, \lambda_{p}=2.5 \mathrm{~cm}, L_{\|}=12.5 \mathrm{~m}$ and with a uniform carbon concentration in the plasma of $1 \%$. The upstream density was ramped from $1 \times 10^{18} \mathrm{~m}^{-3}$ to $1.8 \times 10^{19} \mathrm{~m}^{-3}$ with a constant auxiliary heating power of $1 \mathrm{MW}$ upstream. The onset of detachment was observed by calculating the ion saturation current arriving at the lower inner divertor target plate as the density was increased (figure 5.3). The calculations predict the sheath-limited regime prevails until the density reaches $\approx 5.0 \times$ $10^{18} \mathrm{~m}^{-3}$, followed by a transition to the conduction-limited regime. The onset of detachment, indicated by a saturation and subsequent decrease in the ion saturation current, is found to occur at an upstream density of $\approx 1.0 \times 10^{19} \mathrm{~m}^{-3}$. Without the additional correction terms presented in this section, the onset of detachment is not predicted.

Analysis of the power balance during the density ramp (figure 5.4) indicates an increase in hydrogenic line radiation at higher upstream densities and lower target temperatures. A deficit in the model appears beyond the onset of detachment, where hydrogen line radiation is calculated to decrease as upstream density increases and target temperature decreases, although as $T_{e}<1 \mathrm{eV}$ in this region (not shown), it is beyond the range of validity for this simple model. The quantity of heat convected across the sheath (green curve) is shown to decrease strongly as the upstream density decreases, due to a drop in the target temperature.

The extensions to the two-point model presented here qualitatively describe what 


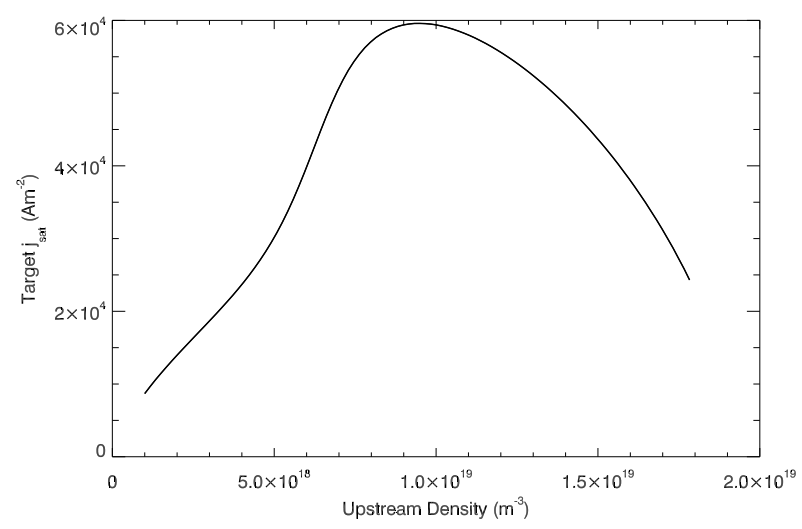

Figure 5.3: Two-point model simulation (equations 5.11-5.14) of the ion saturation current at the lower inner target plate of MAST during a density ramp experiment.

are thought to be the dominant mechanisms that bring about the detached divertor state. The model is able to reproduce some experimentally observations such as a "rollover" in ion saturation current to divertor target plates and increased radiation at the onset of detachment. However, given that it is thought that the dominant physics governing detachment is not fully accounted for and the sensitivity of detachment to the $2 \mathrm{D} / 3 \mathrm{D}$ divertor geometry, the results of this section are intended only as a rough estimate for the detachment threshold.

\subsection{Experimental Observations}

\subsubsection{Operational Limits}

Over the course of this study, divertor detachment has been studied using different fuelling configurations to alter the plasma conditions at the lower inner divertor leg to assess it's effect on the onset and sustainment of detachment. A brief overview of some of the shots examined in this study are given in table 5.1, with plots of some of the pertinent data collected for a subset of these shots in figure 5.7. The onset of detachment was initially ascertained by examining the ratio of $\mathrm{D}_{\gamma}$ and $\mathrm{D}_{\alpha}$ emission from the lower inner strike point (third trace from the top, figure 5.7). This ratio is a common detachment diagnostic, as it is a sensitive indicator of the presence of volume recombination [91] which is a signature of the onset of detachment. The principle behind this diagnostic is illustrated in figure 5.5. When the energy level populations of electrons within ionic energy states are dominated by excitation interactions, the value of $\mathrm{D}_{\gamma} / \mathrm{D}_{\alpha}$ is normally $\approx 1.0 \times 10^{-2}$ with a relatively weak density dependence for densities exceeding $1 \times 10^{19} \mathrm{~m}^{-3}$. In detachment-like conditions $\left(\mathrm{T}_{e}<1 \mathrm{eV}\right)$, recombination processes begin to dominate over excitation, significantly altering the value of this ratio to $\approx 0.1$, an order of magnitude increase. Hence, the ratio of $\mathrm{D}_{\gamma} / \mathrm{D}_{\alpha}$ line intensities 


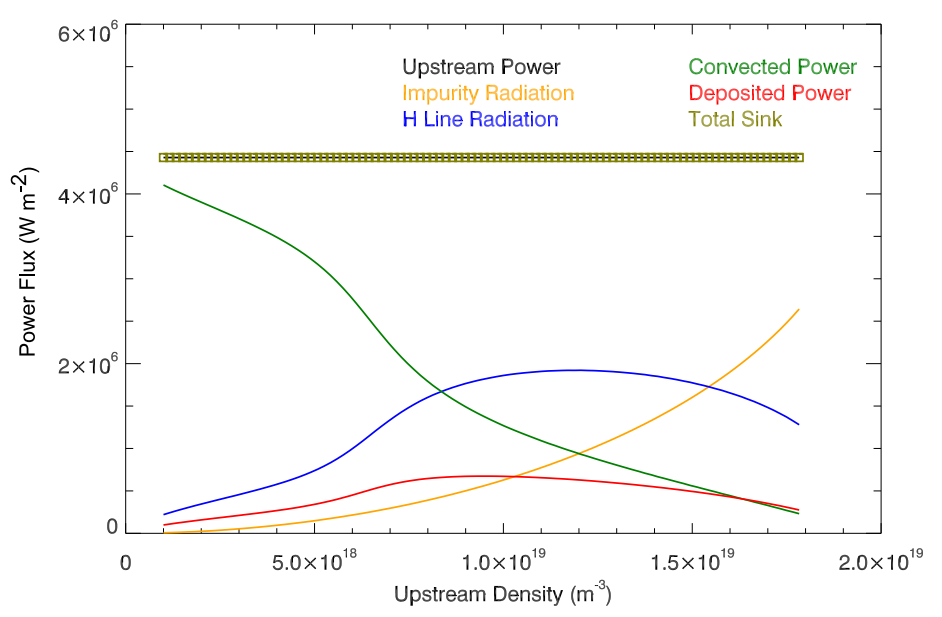

Figure 5.4: Power balance as calculated by a two-point model during a density ramp experiment. The power arriving upstream is transported and lost along field lines by convection and radiated by line radiation by impurities respectively. The onset of detachment is brought about by an increase in hydrogenic line radiation as the target temperature drops.

can be used to detect the presence of recombination mechanisms inherent to detached plasmas, but also offers a means of estimating $\mathrm{T}_{e}$. In shots presented in figure 5.7, the onset of detachment is related to the duration of fuelling from the lower inboard side. Removing this fuelling source in a discharge (shot 24867) causes the plasma to revert to attached conditions (and the onset of $\mathrm{H}$-mode in that case). Moreover, retaining lower inboard gas fuelling throughout the discharge (shot 25028) appears to sustain detachment.

The principal discharges under investigation are shots 25028 and 25029, the latter being similar to the former, with the same discharge parameters. Both discharges had a nominal plasma current of approximately $650 \mathrm{kA}$, the electron density and temperature at the separatrix at the high field side of $1.0 \times 10^{19} \mathrm{~m}^{-3}$ and $10 \mathrm{eV}$ respectively. The core density was held constant for most of the discharge in order to keep the divertor conditions constant throughout the detached phase. This facilitates $2 \mathrm{D}$ line-of-sight spectroscopy measurements as the divertor leg sweeps across the spectrometer viewing chords. 1MW of auxiliary heating was applied using a neutral beam injector. A Hugill diagram [102] of the the discharge evolution is shown in figure 5.6. The discharge begins at the bottom leftmost position on the diagram and proceeds in the direction of increasing Murakami parameter (x-axis). At the onset of detachment, the shot is close to the density limit $[6,103]$, a reflection of the role of divertor detachment in the onset of density limit disruptions [104]. At approximately $240 \mathrm{~ms}$, data from soft X-ray cameras indicated the onset of sawtooth behaviour, indicated by rises and "crashes" in soft X-ray emission. Although not always a fatal instability, sawteeth bring about 

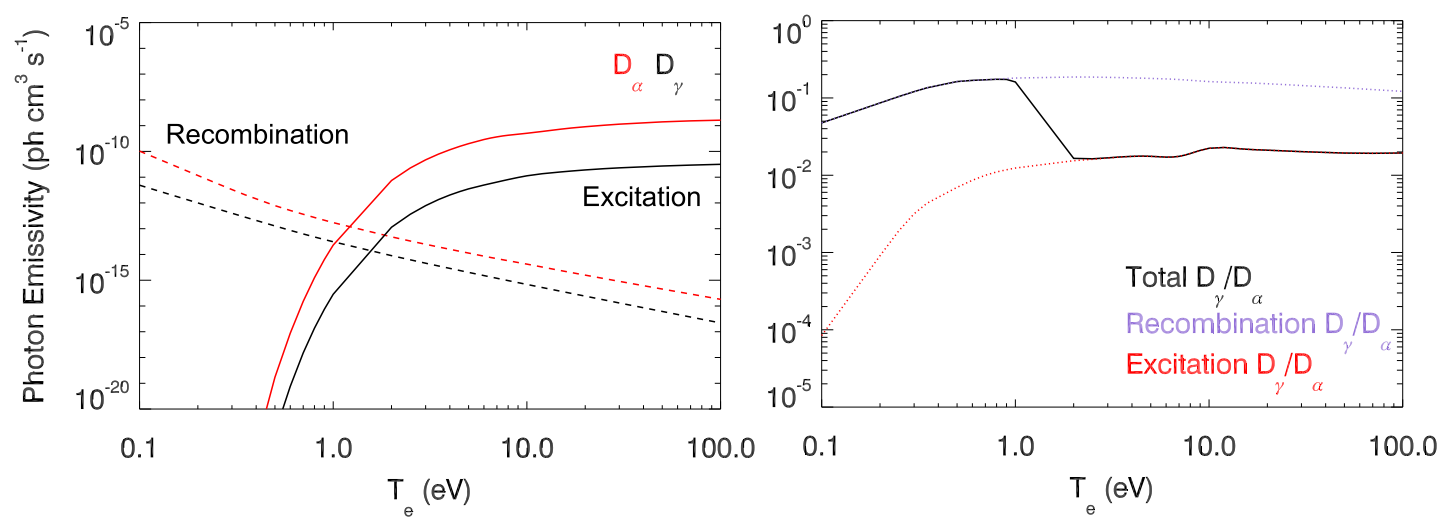

Figure 5.5: Left: photon emissivity coefficients of $\mathrm{D}_{\alpha}$ (red) and $\mathrm{D}_{\gamma}$ (black) line emission due to excitation (solid lines) and recombination (dashed lines) processes. Right: calculation of $\mathrm{D}_{\gamma} / D_{\alpha}$ intensity ratio (black) and from purely excitation (red) and recombination (purple) processes, calculated by the ADAS codes [98], assuming a uniform electron density of $1 \times 10^{19} \mathrm{~m}^{-3}$.

oscillations in temperature and density profiles [105], as a result, data taken during sawtooth activity was not used in this study.

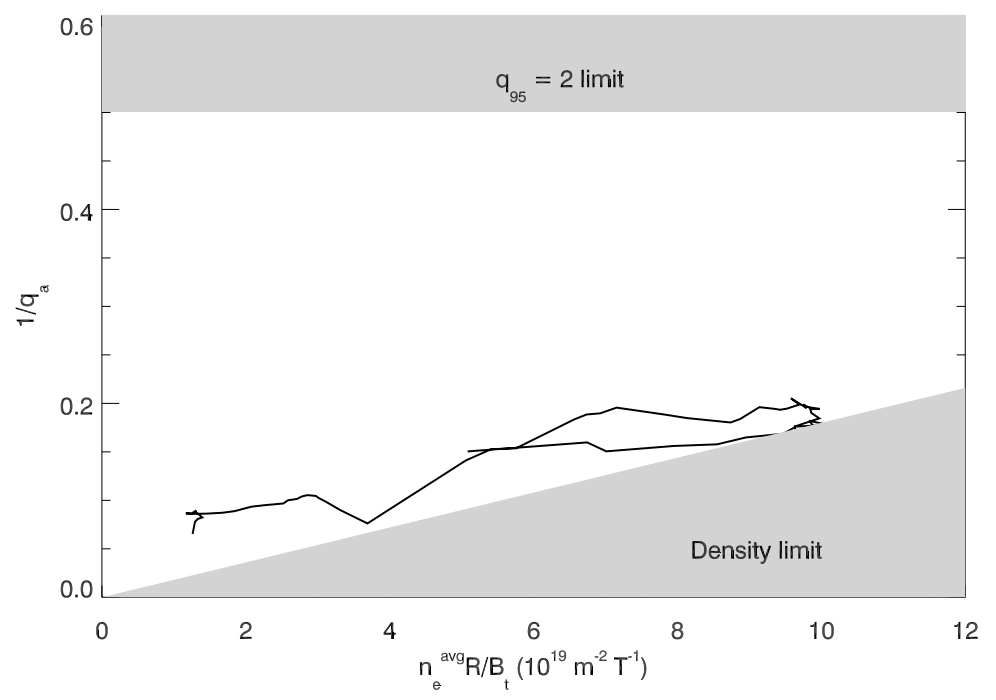

Figure 5.6: Hugill diagram indicating the trajectory of MAST discharge 25028 through parameter space.

\subsubsection{Thomson Scattering}

Upstream density and temperature profiles were measured using a newly upgraded Thomson scattering diagnostic (figure 5.8) [56], that measures such information at approximately $1 \mathrm{~cm}$ resolution, with a nominal temporal resolution of nearly $4 \mathrm{~ms}$. As the diagnostic information collected is input into a time-independent plasma transport 


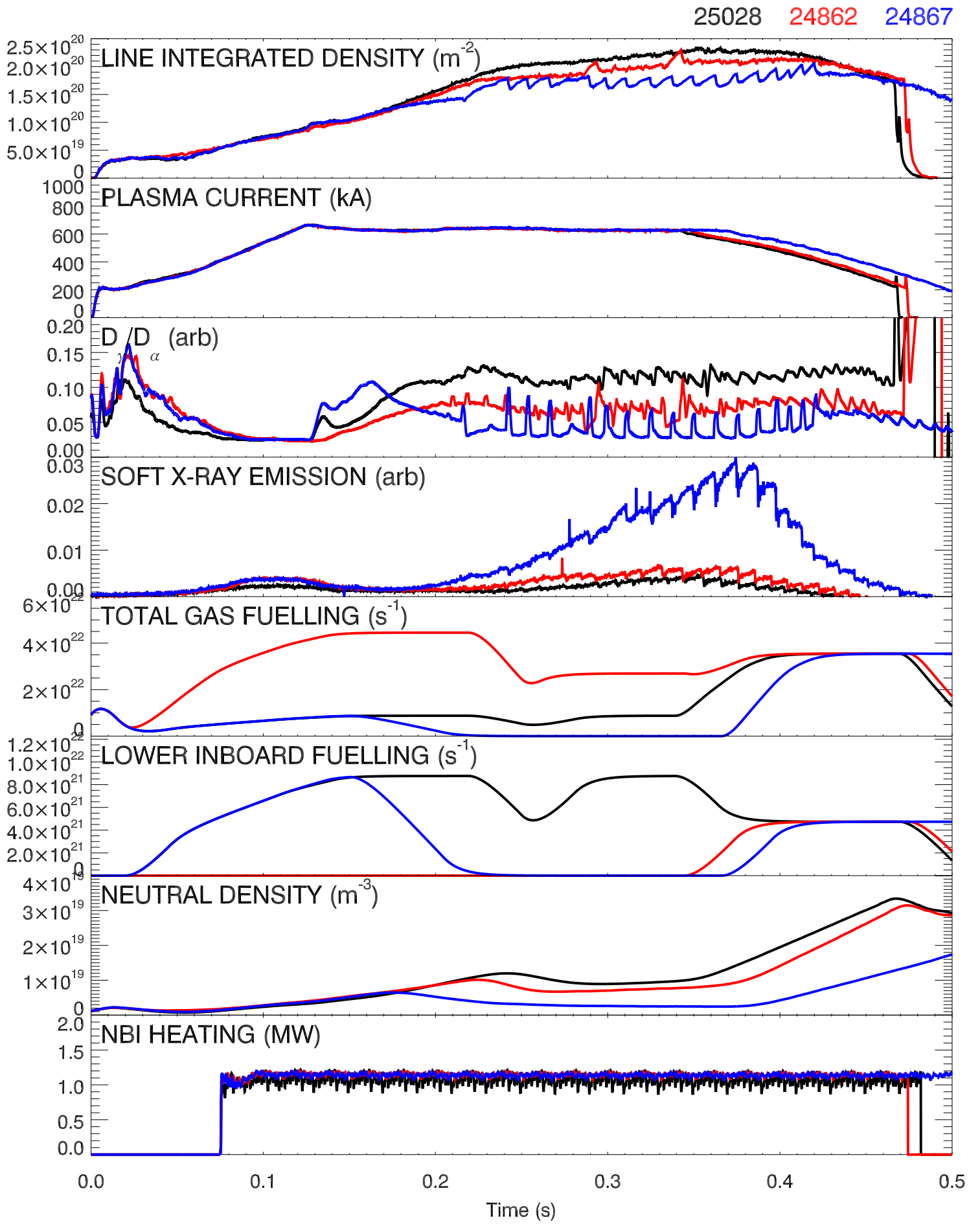

Figure 5.7: Summary of three discharges carried out during this study. Shot 25028 (black) had a detached lower inner divertor leg due to strong $\mathrm{D}_{2}$ puffing in the vicinity of the leg. Shot 24862 (red) was a repeat of shot 24861 (similar to 25028) with inboard gas fuelling turned off at $150 \mathrm{~ms}$. This shot enters a dithering H-mode at $210 \mathrm{~ms}$. Shot 24867 (blue) was fuelled using the outboard gas fuelling system, resulting in the divertor leg being attached throughout the discharge. This shot enters H-mode at 250ms. 


\begin{tabular}{|c|c|c|}
\hline Shot & Description & Data Recorded \\
\hline 24861 & $\begin{array}{c}\text { Detachment at lower inner leg } \\
\text { using } \mathrm{D}_{2} \text { puffing at } 200 \mathrm{~ms}, 1 \mathrm{MW} \text { NBI }\end{array}$ & $\begin{array}{c}\text { Probe data }(100-250 \mathrm{~ms}) \\
\text { DivCam }\left(\mathrm{D}_{\alpha}, \mathrm{D}_{\gamma}\right)\end{array}$ \\
\hline 25028 & Repeat of 24861 & $\begin{array}{c}\text { Probe data }(150-400 \mathrm{~ms}) \\
\text { DivCam }\left(\mathrm{D}_{\alpha}, \mathrm{D}_{\beta}, \mathrm{D}_{\gamma}, \mathrm{D}_{\delta}\right)\end{array}$ \\
\hline 25029 & Repeat of 24861 & $\begin{array}{c}\text { Probe data }(150-400 \mathrm{~ms}) \\
\text { DivCam }\left(\mathrm{CII}, \mathrm{CIII}, \mathrm{D}_{\beta}, \mathrm{D}_{\delta}\right)\end{array}$ \\
\hline 24862 & Repeat 24861, no $\mathrm{D}_{2}$ puffing at 150ms & $\begin{array}{c}\text { Probe data }(100-250 \mathrm{~ms}) \\
\text { DivCam }\left(\mathrm{D}_{\alpha}, \mathrm{D}_{\gamma}\right)\end{array}$ \\
\hline 24869 & Repeat 24862 & $\begin{array}{c}\text { Probe data }(100-250 \mathrm{~ms}) \\
\text { DivCam }(\mathrm{CII}, \mathrm{CIII})\end{array}$ \\
\hline 24866 & Repeat 24861, continually ramping & $\begin{array}{c}\text { Probe data }(100-250 \mathrm{~ms}) \\
\text { DivCam }\left(\mathrm{D}_{\alpha}, \mathrm{D}_{\gamma}\right)\end{array}$ \\
\hline 24867 & Repeat 24861 using outboard fuelling & $\begin{array}{c}\text { Probe data }(100-250 \mathrm{~ms}) \\
\text { only }\end{array}$ \\
\hline 24868 & Repeat 24867 & $\begin{array}{c}\text { Probe data }(100-250 \mathrm{~ms}) \\
\text { DivCam }(\mathrm{CII}, \mathrm{CIII})\end{array}$ \\
& & \\
\hline
\end{tabular}

Table 5.1: Description of the principal discharges in this study.

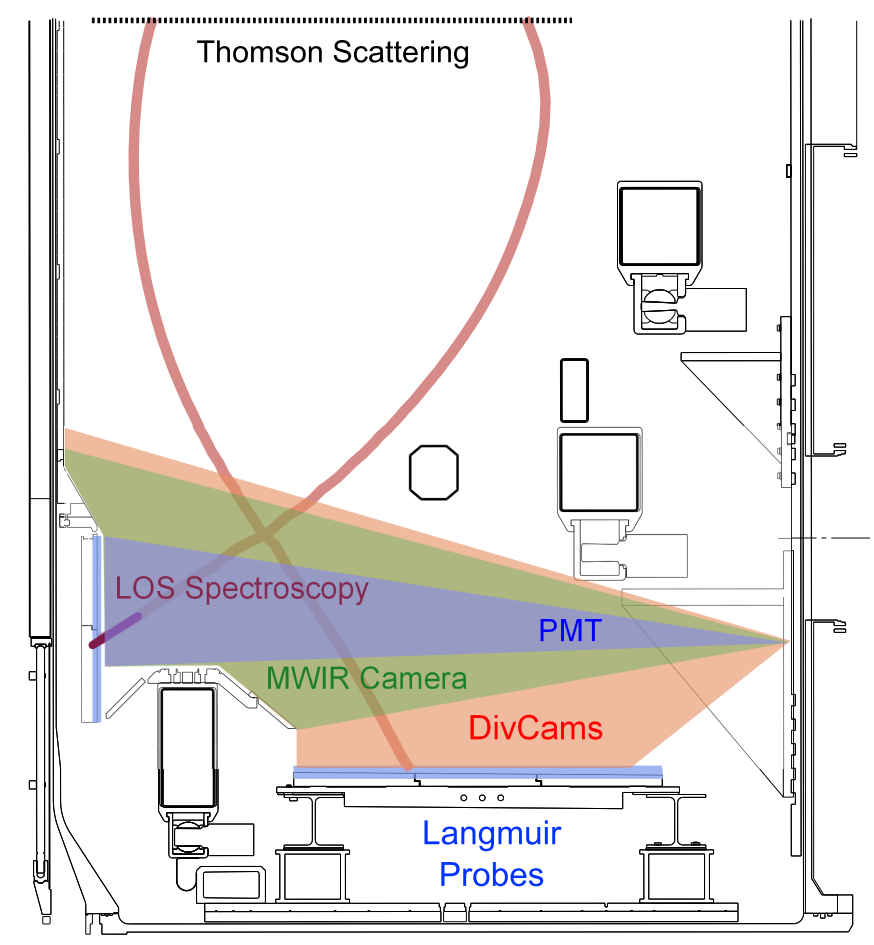

Figure 5.8: Layout of the principal diagnostics used in this study. The diagnostics directed towards the lower divertor were toroidally displaced. 
code, data from before and after the time of interest are collated. The collated data is then filtered to remove any data collected $\pm 1 \mathrm{~ms}$ from sawtooth activity and averaged, producing a radial profile of $n_{e}$ and $T_{e}$ that is input into OSM-EIRENE (figure 5.9). The collation of data from times close to that of interest increases the number of data points used in the averaging process and thereby increases the quality of the dataset. Data collected from the high-field side midpane is used in this study due to it's close proximity to the detached region, thereby placing a stronger constraint on the OSMEIRENE plasma solution where it is needed most. Furthermore, there is reduced scatter in the data at the high-field side, helped by measurements here being in a region of positive magnetic field curvature [106].

\subsubsection{Target Langmuir Probes}

Measurements of ion saturation current were recorded at the lower inner and outer divertor target plates at a spatial resolution of $6 \mathrm{~mm}$ and $9 \mathrm{~mm}$ respectively and temporal resolution of approximately $1 \mathrm{~ms}$, as described in section 3.3.1. During the design of the detachment shot, the divertor P2 coil waveform was optimised to keep the lower inner strike point within the coverage of the Langmuir probes for as long as possible. This became necessary as the $\mathrm{P} 1$ solenoid flux swing causes the strike point to "sweep" upwards in the case of the lower inner strike point, at a rate of $\approx 1 \mathrm{~ms}^{-1}$. Data was recorded for longer than the detached phase of discharges 25028 and 25029, from $150 \mathrm{~ms}$ to $400 \mathrm{~ms}$. Data was only recorded over the period when the plasma struck a sufficient number of probes for measurements in both the near-SOL and private flux regions. Furthermore, no data was used that was in the vicinity of sawtooth activity.

The onset of detachment manifests as a strong reduction in ion flux to the lower inner strike point within a region between the private flux region and the separatrix, as shown figure 5.10. Accurate assessment of the location in ion flux using Langmuir probe data alone is difficult due to spatial resolution of the probes combined with the flux mapping calculated by EFIT. The outer strike point however appears attached, with no noticeable reduction in ion flux to the target plate. The inner target plate profile is indicative of partially detached conditions, where the region of reduced ion flux is confined to a region close to the private flux region. Similar ion flux profiles were observed in a prior detachment study on MAST [90], although on other machines such as Alcator C-Mod, the reduction in ion flux to the target plate was less well localised [107] and manifests as a reduction in the overall ion flux profile. Target electron temperature data was not the subject of further analysis as research on other Tokamaks have demonstrated inconsistency between Thomson scattering [108] and spectroscopy [109] and Langmuir probe $\mathrm{T}_{e}$ measurements, where the latter is believed to be the less accurate measurement. Furthermore, the set-up of the MAST Langmuir probe data 


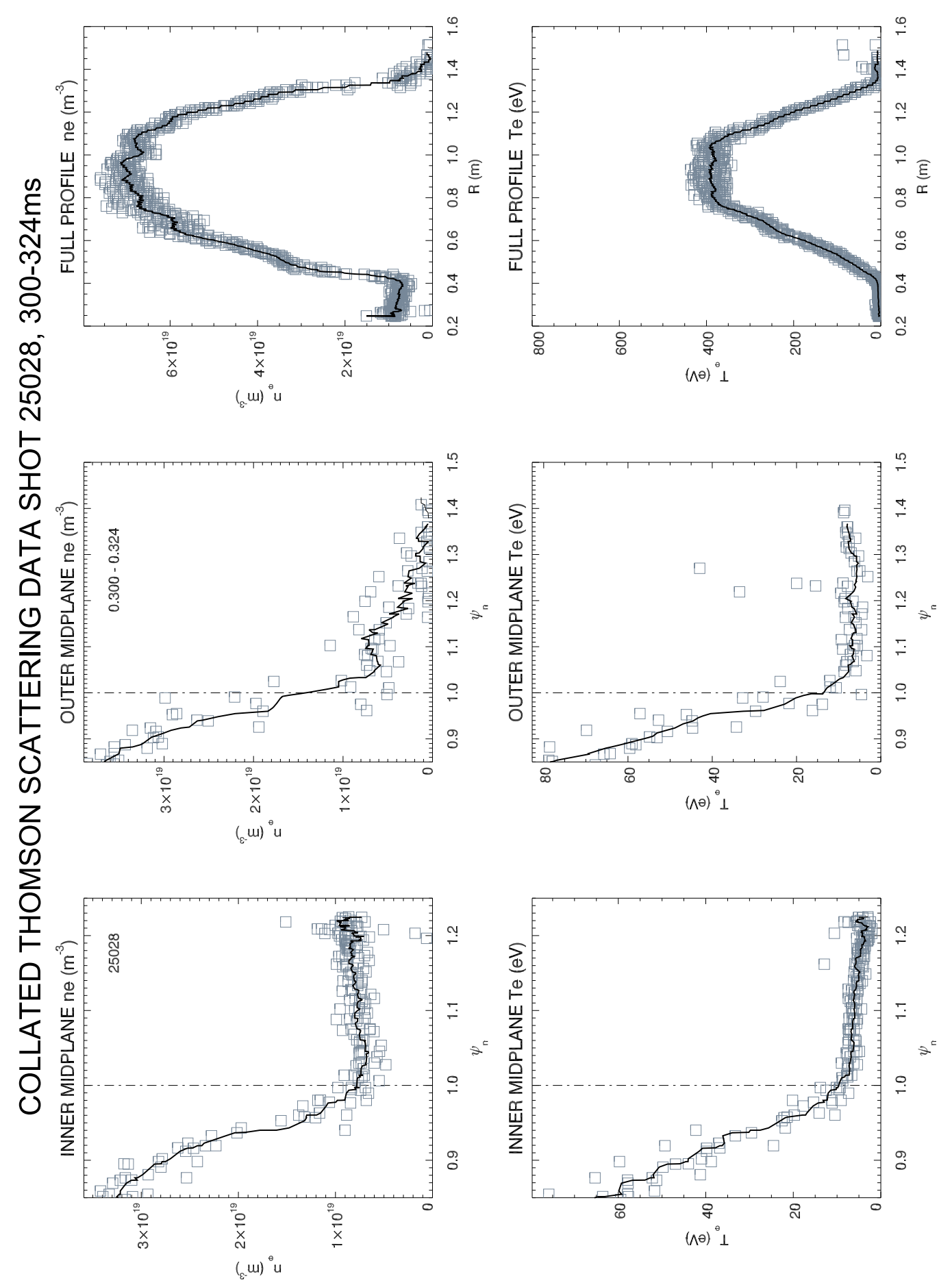

Figure 5.9: Thomson scattering data used to constrain an OSM-EIRENE simulation of partially detached discharge 25028 at $300-324 \mathrm{~ms}$. 
acquisition limits the minimum resolvable electron temperature to $3-5 \mathrm{eV}$ [110], which is above the electron temperatures expected in detachment.

An interesting feature of the probe data at both targets is how little the profiles vary with time. This is clearly advantageous from a modelling point of view, as the Langmuir probe data does not constrain the time within the shot it is best to carry out a simulation run. The data input into OSM-EIRENE was sampled from shot 25028 at $300-325 \mathrm{~ms}$, with the raw and smoothed profiles shown in figure 5.11.
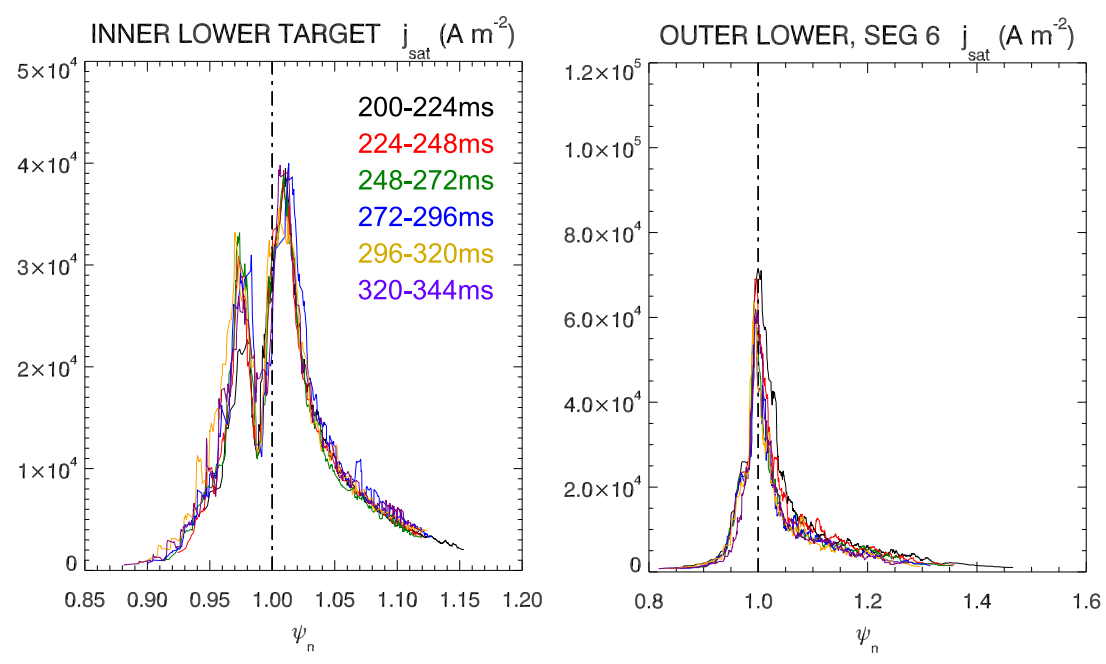

Figure 5.10: Langmuir probe ion saturation current data recorded at the lower inner and outer strike points during shot 25028. The data appear to vary little during the detached phase, permitting the use of time-averaged data in interpretive modelling.

\subsubsection{InfraRed Thermography}

A filtered $(4.5-5 \mu \mathrm{m})$ IR (Medium Wavelength - MWIR) camera was used to observe the lower inner strike point and in a region that intersects the lower inner divertor leg. The information collected (figure 5.12) shows two emission bands at the lower inner strike point, supporting observations made by Langmuir probes of a reduction in ion flux at the strike point. Furthermore, the divertor leg can also be observed with this camera. This behaviour has not been observed before with this camera on MAST, and it is believed to be caused by broad-spectrum Bremsstrahlung emission generated by strong $\mathrm{D}_{2}$ puffing in the vicinity of the divertor leg, section 3.3.6. If this gas puffing is not used, as in the case of shot 24862, this emission from the divertor leg is not observed. Consequently, it is not possible to accurately measure the divertor heat flux evolution during a detached discharge. 

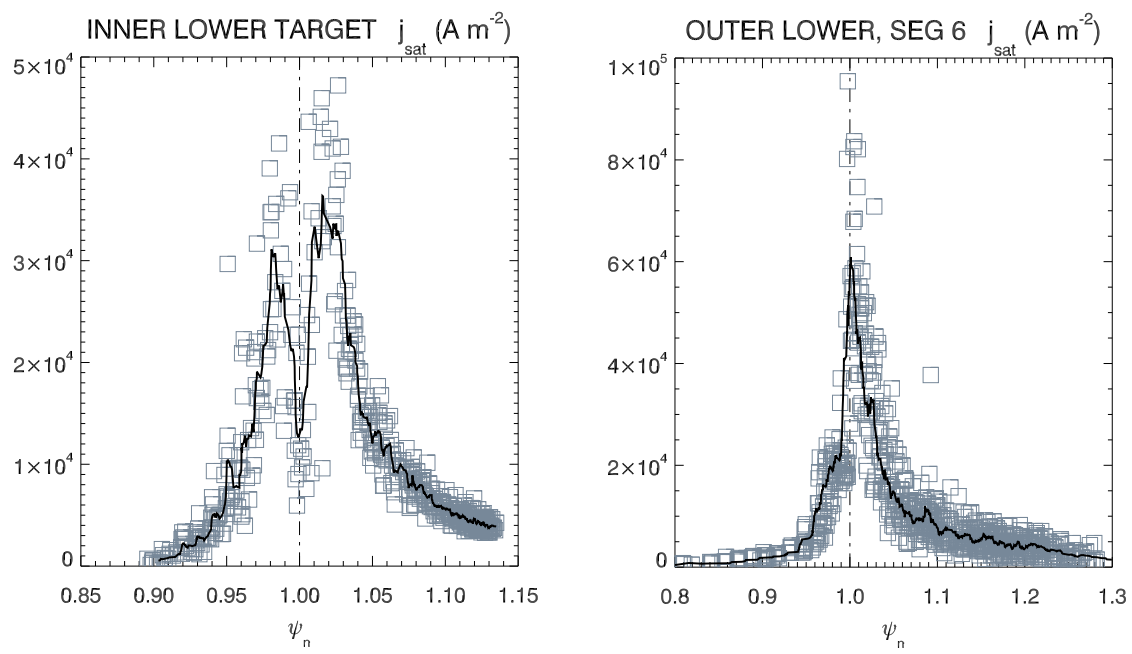

Figure 5.11: Time-averaged Langmuir probe data input into OSM-EIRENE. The data was collated from shot 25028 from 300 to $325 \mathrm{~ms}$, where the black line, which is a moving average, is input into the code.
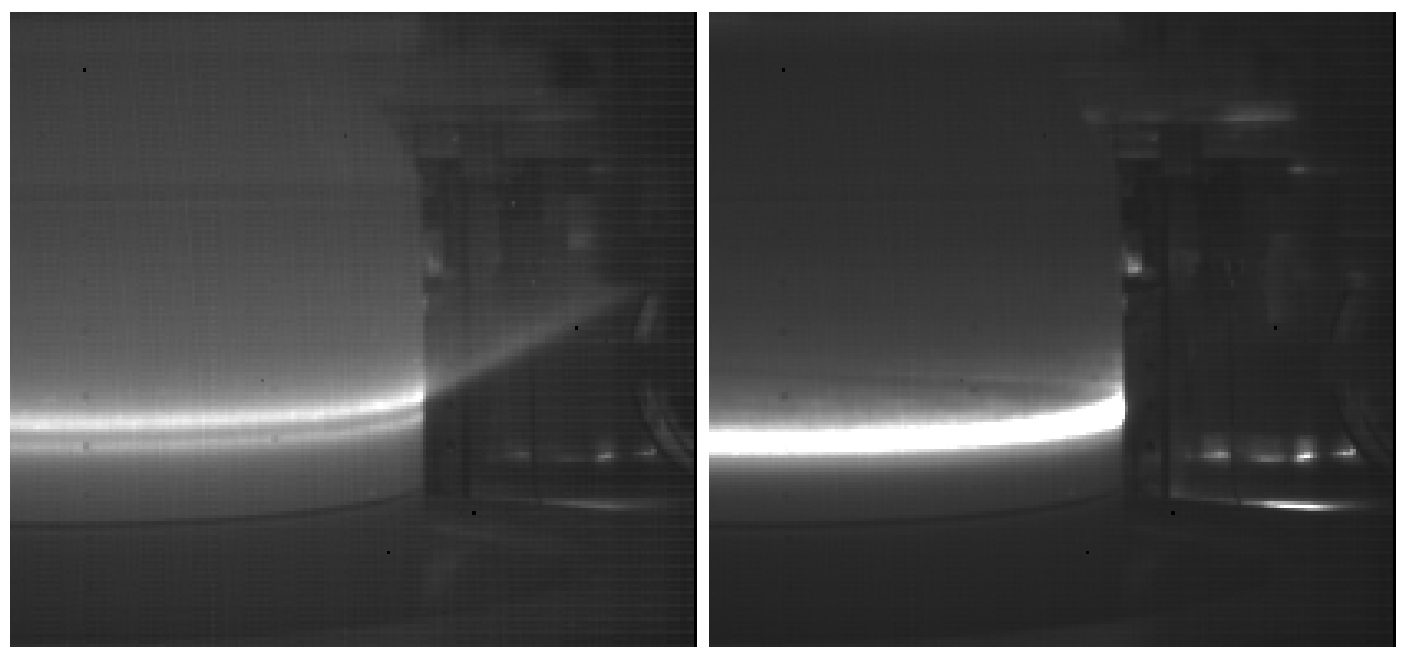

Figure 5.12: Left: frame recorded from the MWIR camera from shot 24861 at $312 \mathrm{~ms}$. Two emission peaks can be seen at the strike point, confirming observations made with the Langmuir probes. The divertor leg can also be clearly seen. This does not occur when $\mathrm{D}_{2}$ puffing close to the divertor leg is turned off, as in shot 24862 at the same time (right). 


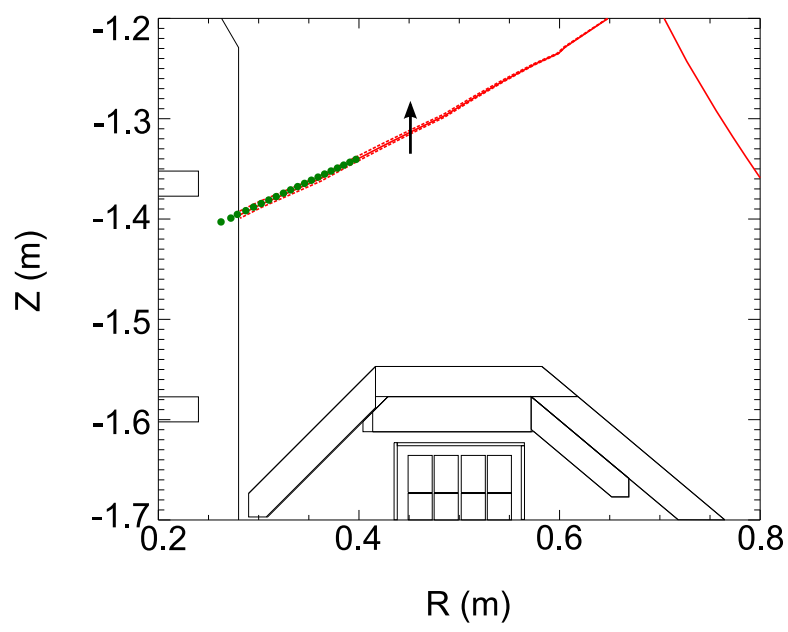

Figure 5.13: Spectroscopic view tangency locations (green) directed at the lower inner divertor leg, as shown in an EFIT reconstruction (red), calculated in the middle of a spectrometer's acquisition cycle. The dashed red lines indicate the EFIT calculated divertor leg locations at the start and end of the acquisition cycle, with a black arrow indicating the strike point sweeping direction.

\subsubsection{Divertor Line-of-Sight Spectroscopy}

As described in chapter 3, two high-resolution spectrometers were used to measure line integrated density and line averaged temperature of plasma in the recombining region. The vessel-facing optics were positioned such that the line of optical fibres were aligned along the separatrix flux tube during the recombining phase of the discharge (figure $5.13)$, as this permits measurements of the electron static pressure balance along the flux tube. Furthermore, measurements of adjacent flux tubes are made possible as the divertor leg sweeps past the spectrometer lines of sight. To facilitate this measurement, the divertor plasma conditions were not altered throughout the detached phase.

As divertor detachment was initiated by $\mathrm{D}_{2}$ puffing in the proximity of the region of interest, contamination of spectroscopic data by broad-spectrum molecular emission was a concern. This emission manifested as a wavelength-dependent baseline, which was subtracted from absolutely (intensity) calibrated data [46] by measuring the baseline signal in the line-free regions $382-386 \mathrm{~nm}, 392-395 \mathrm{~nm}$ and $400-408 \mathrm{~nm}$ (figure 5.14, two regions shown for clarity) and fitted the baseline to a quadratic function to subtract this baseline from the spectrum. The resultant spectrum was then interpreted as outlined in section 3.3.3 to determine line-averaged electron temperatures along the lines of sight. This analysis was carried out for 15 out of 20 total lines of sight using the available $300 \mathrm{~mm}$ focal length spectrograph. Measurements using the $1000 \mathrm{~mm}$ spectrograph, where 5 vessel-facing fibres were input, were unable to determine $\mathrm{T}_{e}$ due to limited spectral range $(\approx 12 \mathrm{~nm})$. The measured $\mathrm{T}_{e}$ profile when the lower divertor leg was 
coincident with the spectrometer lines of sight (figure 5.15) reveal a profile with little variation along the leg. This observation is consistent with others made at DIII-D [111].

Line integrated electron densities were measured by measuring the shape of the $\mathrm{D}_{\epsilon}$ line $(397 \mathrm{~nm})$ and fitting observed line shapes to convolutions of measured instumental broadening and theoretical Stark broadened profiles [45], as described in section 3.3.4. Measurements made on earlier MAST discharges found that line integrated density measurements made using only the $\mathrm{D}_{\epsilon}$ line agreed, to within experimental uncertainties, with observed densities calculated using observations of the shapes of $\mathrm{D}_{\eta}(383.5 \mathrm{~nm})$ and $\mathrm{D}_{\theta}(379 \mathrm{~nm})$. Measurements of $\mathrm{D}_{\zeta}(389 \mathrm{~nm})$ line shapes, although measured by both spectrometers during shot 25028 , could not be used in the data analysis procedure due to the presence of a nearby He recombination line that could not be resolved from the line of interest. Data from all 5 channels in the $1000 \mathrm{~mm}$ focal length spectrometer and 4 out of 18 channels in the $300 \mathrm{~mm}$ focal length spectrometers were used in this analysis. Off-axis aberrations in the $300 \mathrm{~mm}$ instrument degraded spectral resolution and resulted in asymmetrical line shapes beyond the four most on-axis chords, preventing this form of analysis being carried out. The density profile measured during shot 25028 is shown in figure 5.15. The density profile increases with increasing proximity to the divertor target plate, clearly indicating a loss of pressure balance along field lines, consistent with the definition of divertor detachment. These observations, however, suggest that plasma pressure increasing with decreasing distance to the divertor target plate, as opposed to decreasing, as is normally anticipated $[7,8,18]$. However, the dynamic, i.e. $m_{i} n_{i} v_{i}^{2}$, pressure is unknown along the divertor leg, and therefore the total parallel pressure balance is unknown. The effect of photon reflections from the centre column and variations in plasma emission over the lines of sight also introduce an additional uncertainty in spectroscopic measurements of $n_{e}, T_{e}$ in close proximity to the strike point. The former explanation is likely to only have a weak effect on the measured signal since the centre column is lined with graphite. The latter effect may explain the line integrated density data point at $\mathrm{R}=0.365 \mathrm{~m}$, where the density is higher than expected, but the line of sight is furthest away from any surfaces. These sources of uncertainty have not been accurately assessed, and future work is required to better understand how they affect the measurements made in this study. Furthermore, the temporal resolution of the AM-510 and SP2300i spectrometer detectors were 20ms and $14 \mathrm{~ms}$ respectively, and hence take spectra during sawtooth activity. This prohibits the filtering of data to remove the effects of sawteeth, which will reduce the plasma emissivity of high-n Balmer emission lines as a heat pulse from the sawtooth temporarily raises $T_{e}$ and reduces the rate of ion-electron recombination reactions and reducing the measured signal. As a result, the effects of sawtooth activity is relatively mild on this diagnostic, which is less sensitive to plasma conditions during a sawtooth crash, and so 


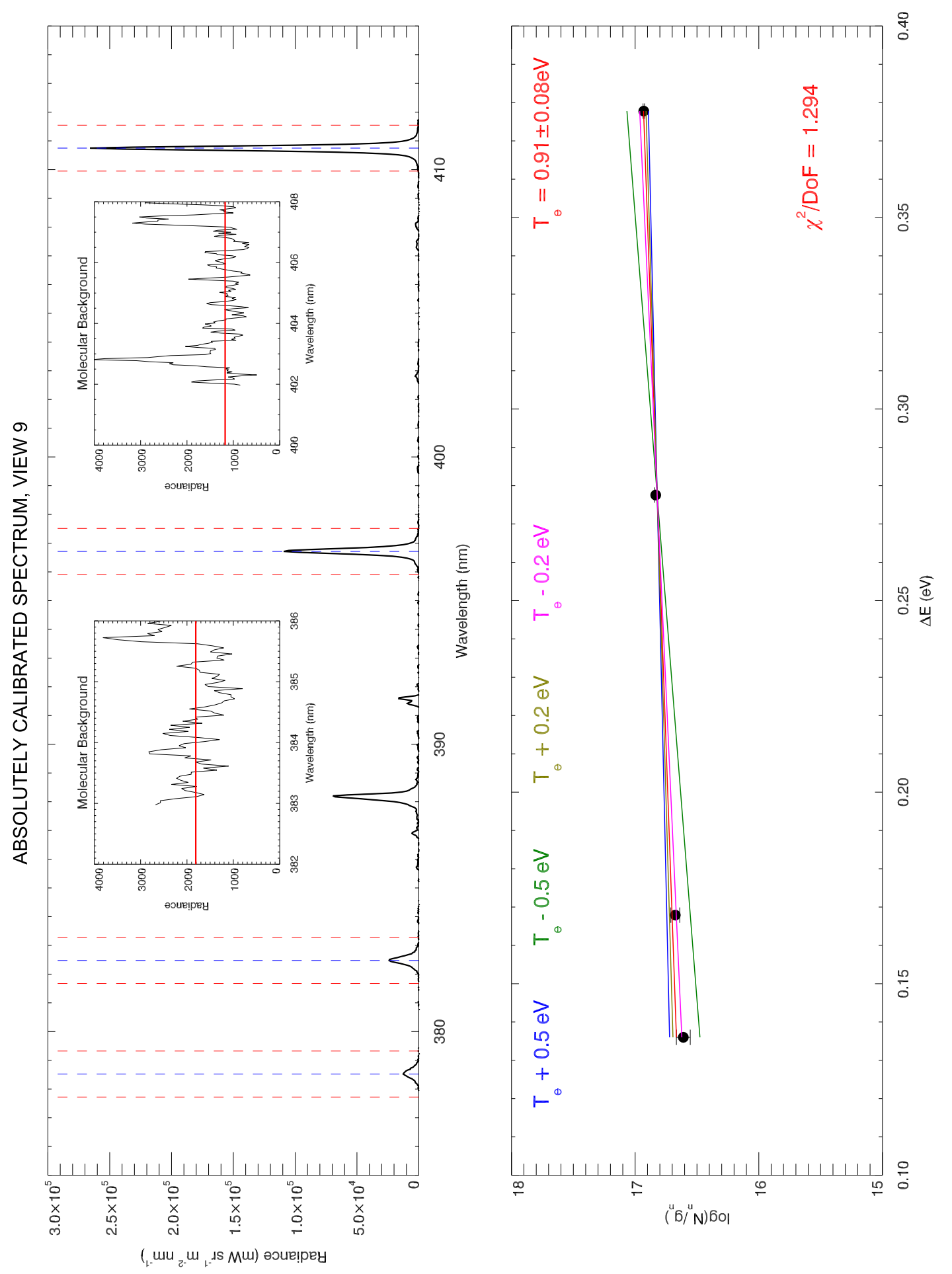

Figure 5.14: Raw data analysis to determine electron temperature from Balmer line ratios using data from the spectrometer line of sight adjacent to the lower inner strike point. The average baseline was calculated in line-free regions to reduce the effects of broadband emission. 


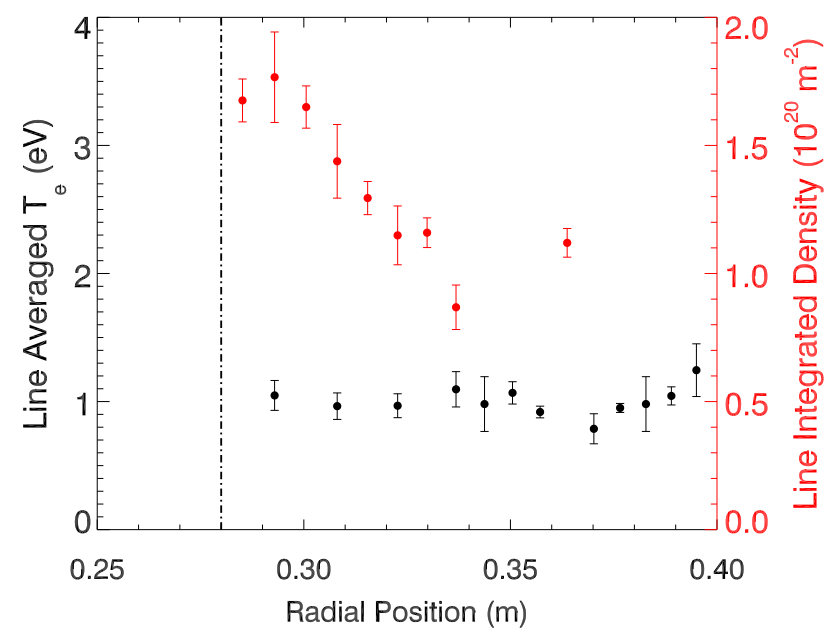

Figure 5.15: Line averaged electron temperature and line integrated density measurements during the recombining phase of shot 25028, measured using line-of-sight spectroscopy of high-n Balmer emission lines. Radial location indicates the R coordinate of the view used (figure 5.13). The strike point is indicated by a dashed vertical line.

the data will be "weighted" towards the detached plasma conditions.

The data collected was plotted as a function of vertical distance between the chord tangency location and that of the separatrix flux tube, as calculated by EFIT, to facilitate interpolation of the density and temperature data where the separatrix was exactly in the centre of the spectrometer viewing chord. This data was then input into the OSM-EIRENE code for further analysis.

\subsubsection{Imaging Spectroscopy}

Narrow-bandwidth imaging spectroscopy (section 3.3.7) has been used during the onset and sustainment of detachment to determine the spatial structure and evolution of divertor spectral line emission. The principal spectral lines of observed and the filter bandwidth used (full-width at half-maximum, $\mathrm{nm})$ are $\mathrm{D}_{\alpha}(656 \mathrm{~nm}, 3 \mathrm{~nm}) \mathrm{D}_{\gamma}(434 \mathrm{~nm}$, $1.5 \mathrm{~nm})$, CII $(514 \mathrm{~nm}, 3 \mathrm{~nm})$ and CIII $(465 \mathrm{~nm}, 1.5 \mathrm{~nm}) . \mathrm{D}_{\alpha}$ and $\mathrm{D}_{\gamma}$ emission intensities are useful constraints for simulation codes modelling detachment, as they are sensitive to volume recombination processes [91,112]. Data was also collected using $98 \mathrm{~Hz}$ frame rate cameras of $\mathrm{D}_{\beta}(486 \mathrm{~nm}, 1.5 \mathrm{~nm})$ and $\mathrm{D}_{\delta}(410 \mathrm{~nm}, 5 \mathrm{~nm})$ emission using the remaining DivCam channel with Imperx IPX-1M48-L cameras. CII and CIII line intensities are sensitive to $\mathrm{T}_{e}$ (figure 5.16) and hence can be used to constrain OSM electron temperature profiles. The $\mathrm{D}_{\alpha}, \mathrm{D}_{\gamma}, \mathrm{CII}$ and CIII data was recorded using a Photron APX-RS camera with a $5 \mathrm{kHz}$ frame rate, and telecentric optics allowing a spatial resolution of approximately $3 \mathrm{~mm}$. The recorded information of plasma emission at the 
lower inner strike point was subsequently tomographically inverted, to calculate the spectral emission from a poloidal cross-section of the plasma, at a spatial resolution of approximately $5 \mathrm{~mm}$. Data from the outer strike point was also recorded, but was not further interpreted, as toroidal discontinuities in the divertor structures degrade the quality of tomographic inversions [60].

For example, the onset of detachment as seen in CII emission (figure 5.20) manifests as a reduction in emission at the vicinity of the strike point, as the plasma in this region cools. During the detached phase, the lack of CII emission in the vicinity of the strike point imposes an upper bound on the local electron temperature, $\mathrm{T}_{e} \leq 1 \mathrm{eV}$ due to a rapid reduction in the CII species below this temperature (figure 5.16). Similarly, ADAS data can be used to infer that the CIII emission zone lies roughly within $3 \mathrm{eV} \leq \mathrm{T}_{e}$ $\leq 10 \mathrm{eV}$, allowing $\mathrm{T}_{e}$ contours to be formed at the periphery of these zones. The evolution of detachment of the inner divertor leg results in movement of CII and CIII emission zones away from the strike point towards the x-point (figure 5.17), indicating a reduction in $\mathrm{T}_{e}$ along the divertor leg with time. The effects of sawtooth behaviour on the data analysis was mitigated by not using frames within $\pm 1 \mathrm{~ms}$ of a sawtooth crash.

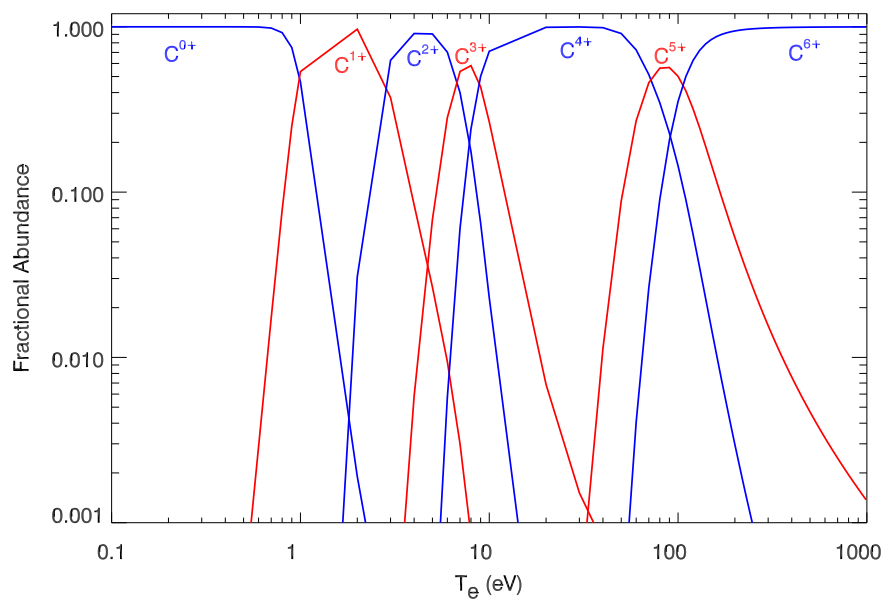

Figure 5.16: The fractional abundance of carbon charge states as a function of plasma electron temperature, using data from the ADAS codes [98].

The interpretation of $\mathrm{D}_{\alpha}$ and $\mathrm{D}_{\gamma}$ data is complicated by high $\mathrm{D}_{2}$ densities brought about by strong puffing in the vicinity of the divertor leg. Consequently, molecular processes enhance $\mathrm{D}_{\alpha}$ and $\mathrm{D}_{\gamma}$ emission, although these processes are a significantly larger contribution to $\mathrm{D}_{\alpha}$ than $\mathrm{D}_{\gamma}$. Figure 5.30 shows the results of an EIRENE simulation of shot 25028 at $312 \mathrm{~ms}$, where the contribution of different atomic and molecular processes contribute to $\mathrm{D}_{\alpha}$ and $\mathrm{D}_{\gamma}$ emissivities. It can be seen that the molecular contribution to $\mathrm{D}_{\alpha}$ is stronger than that of $\mathrm{D}_{\gamma}$ emission. In terms of analysing this data in terms of line ratios of $\mathrm{D}_{\gamma} / \mathrm{D}_{\alpha}$ intensities, molecular processes will reduce 


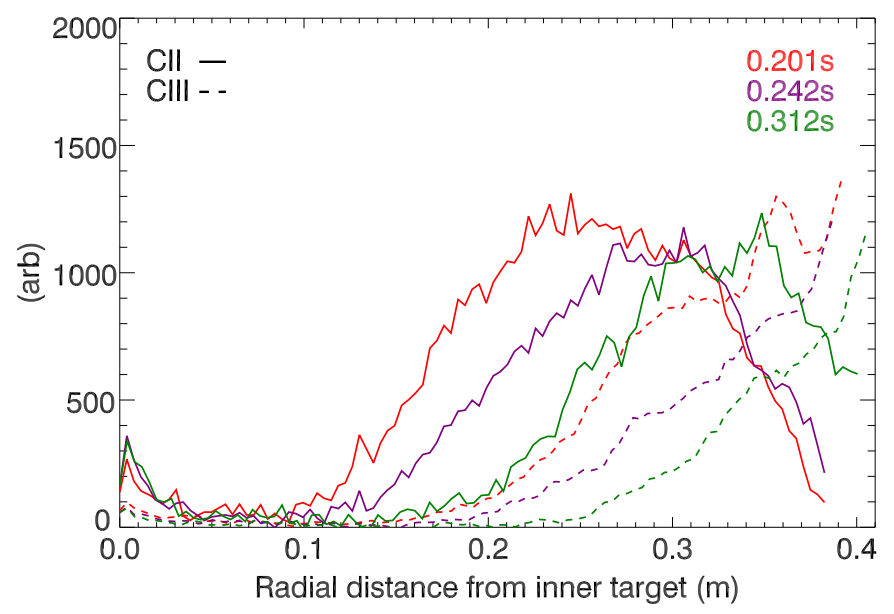

Figure 5.17: The evolution of CII and CIII emission during the detached phase of shot 25029. The data plotted is light intensity along the separatrix, as shown in 5.21.

such line ratios and volumetric recombination processes will increase them. Narrowbandpass imaging of $\mathrm{D}_{\alpha}$ and $\mathrm{D}_{\gamma}$ lines during the detached phase of shot 25028 (figures $5.18,5.19$ ) indicate the presence of a recombining region (high $\mathrm{D}_{\alpha}$ and $\mathrm{D}_{\gamma}$ intensity) in the vicinity of the strike point. Between the x-point and the strike point, there is a region of elevated $D_{\alpha}$ emission, which is thought to be a consequence of strong $\mathrm{D}_{2}$ puffing in this region (figure 5.18) resulting in more molecular dissociation events occurring.

Comparison of $\mathrm{D}_{\alpha}$ and $\mathrm{D}_{\gamma}$ line intensities along the inner divertor leg (figure 5.22) have been computed by relatively calibrating the camera data such that, in attached conditions, the ratio of $\mathrm{D}_{\alpha}$ and $\mathrm{D}_{\gamma}$ line intensities are unity. This allows the presence of volume recombination processes to be determined when $\mathrm{D}_{\gamma} / \mathrm{D}_{\alpha}>1$ (figure 5.5). The calibration was carried out using data from shot 24867 at $430 \mathrm{~ms}$, which is similar to shots 25028 and 25029, but the fuelling location was changed to the low-field side. Consequently, plasma entered H-mode and the divertor plasma displayed no signs of detachment. Calibration data was taken between ELM bursts to reduce the influence of these transients on the calibration. The attached plasma calibration data (blue trace in figure 5.22) shows a departure from $\mathrm{D}_{\gamma} / \mathrm{D}_{\alpha}=1$ in the vicinity of the strike point ( $\mathrm{R}$ $\approx 0.01 \mathrm{~m}$ ) possibly due to the presence of localised carbon line emission in this region, within the bandpass of the $\mathrm{D}_{\gamma}$ filter used in this study (figure 5.23).

The detached plasma data (figure 5.22, black trace) indicate a strong departure from attached conditions in the vicinity of the strike point $(\mathrm{R} \approx 0.1 \mathrm{~m})$, with elevated $\mathrm{D}_{\gamma}$ emission suggesting volumetric recombination processes are taking place. Towards the x-point, the plasma appears to be mostly hotter and in an excitation-dominated regime, indicated by $\mathrm{D}_{\gamma} / \mathrm{D}_{\alpha}=1$. 


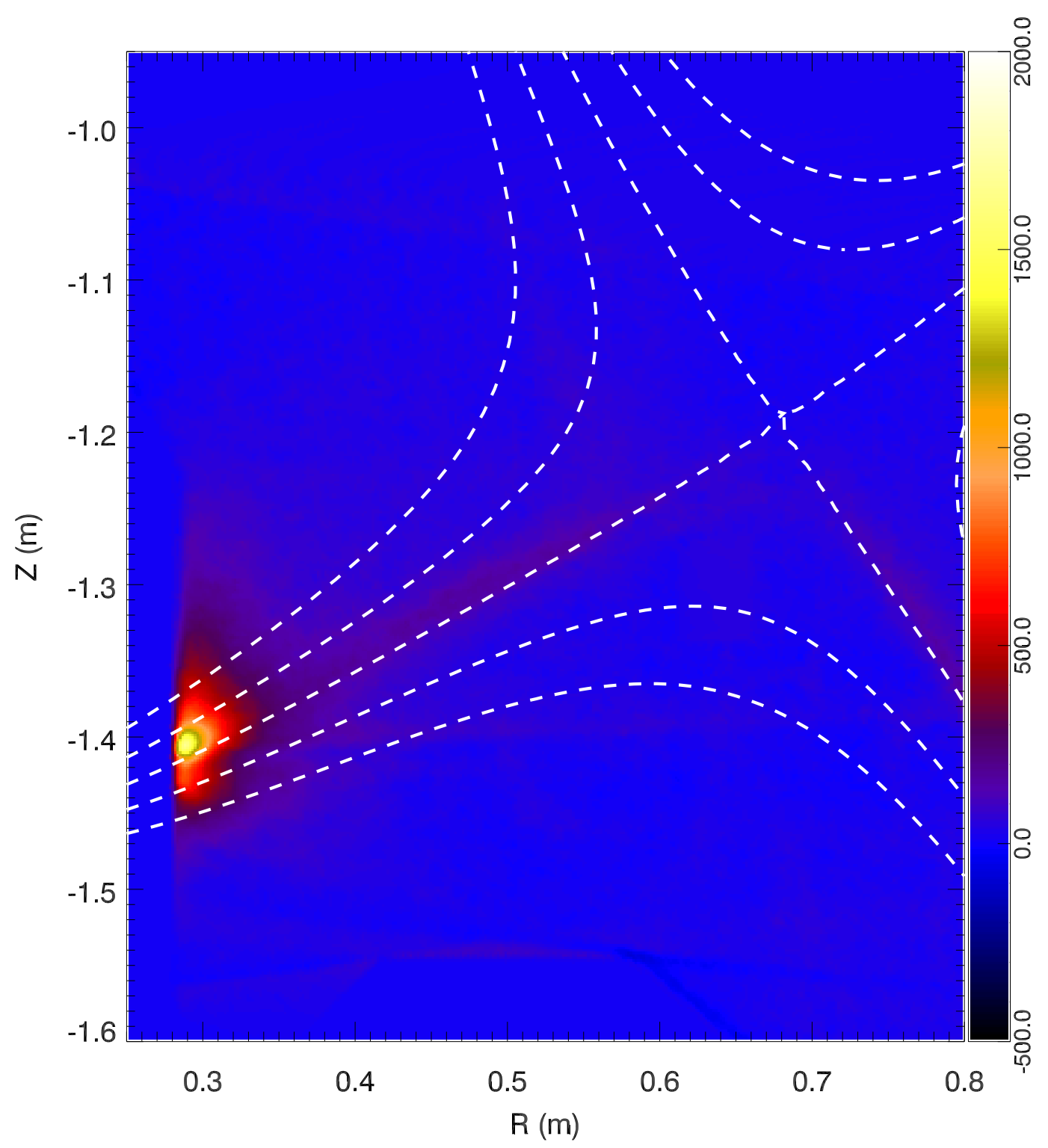

Figure 5.18: Tomographically inverted $\mathrm{D}_{\alpha}(656 \mathrm{~nm})$ data from shot 25028 at $312 \mathrm{~ms}$, concentrating on the inner divertor leg with a magnetic equilibrium from EFIT. 


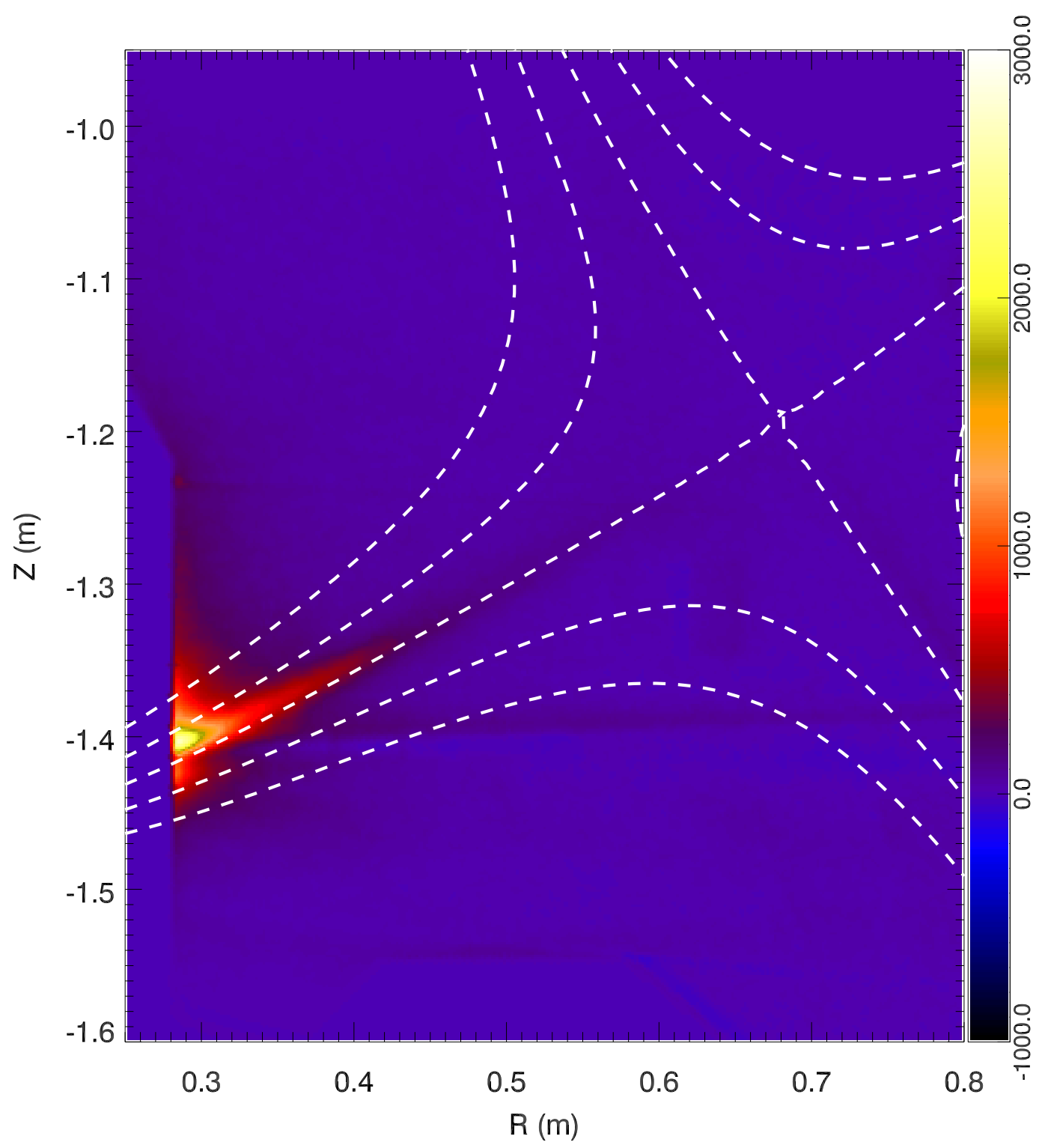

Figure 5.19: Tomographically inverted $\mathrm{D}_{\gamma}(434 \mathrm{~nm})$ data from shot 25028 at $312 \mathrm{~ms}$, concentrating on the inner divertor leg with a magnetic equilibrium from EFIT. Unlike $\mathrm{D}_{\alpha}$ data, there is an emission peak close to the $\mathrm{D}_{2}$ gas puffing location at $\mathrm{R}=0.28 \mathrm{~m}$, $\mathrm{Z}=-1.23 \mathrm{~m}$. 


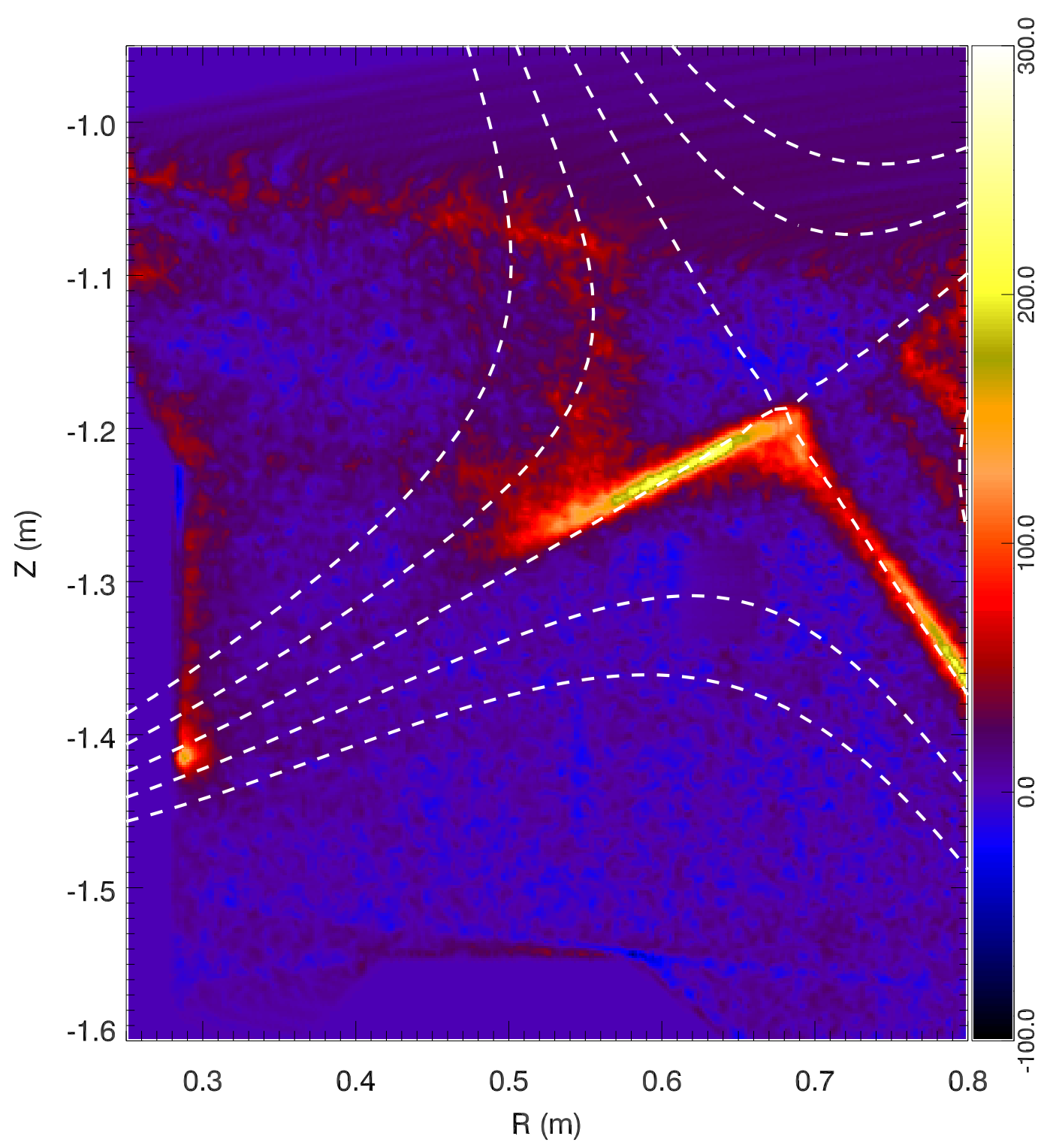

Figure 5.20: Tomographically inverted CII (514nm) data from shot 25028 at $312 \mathrm{~ms}$, concentrating on the inner divertor leg with a magnetic equilibrium from EFIT. 


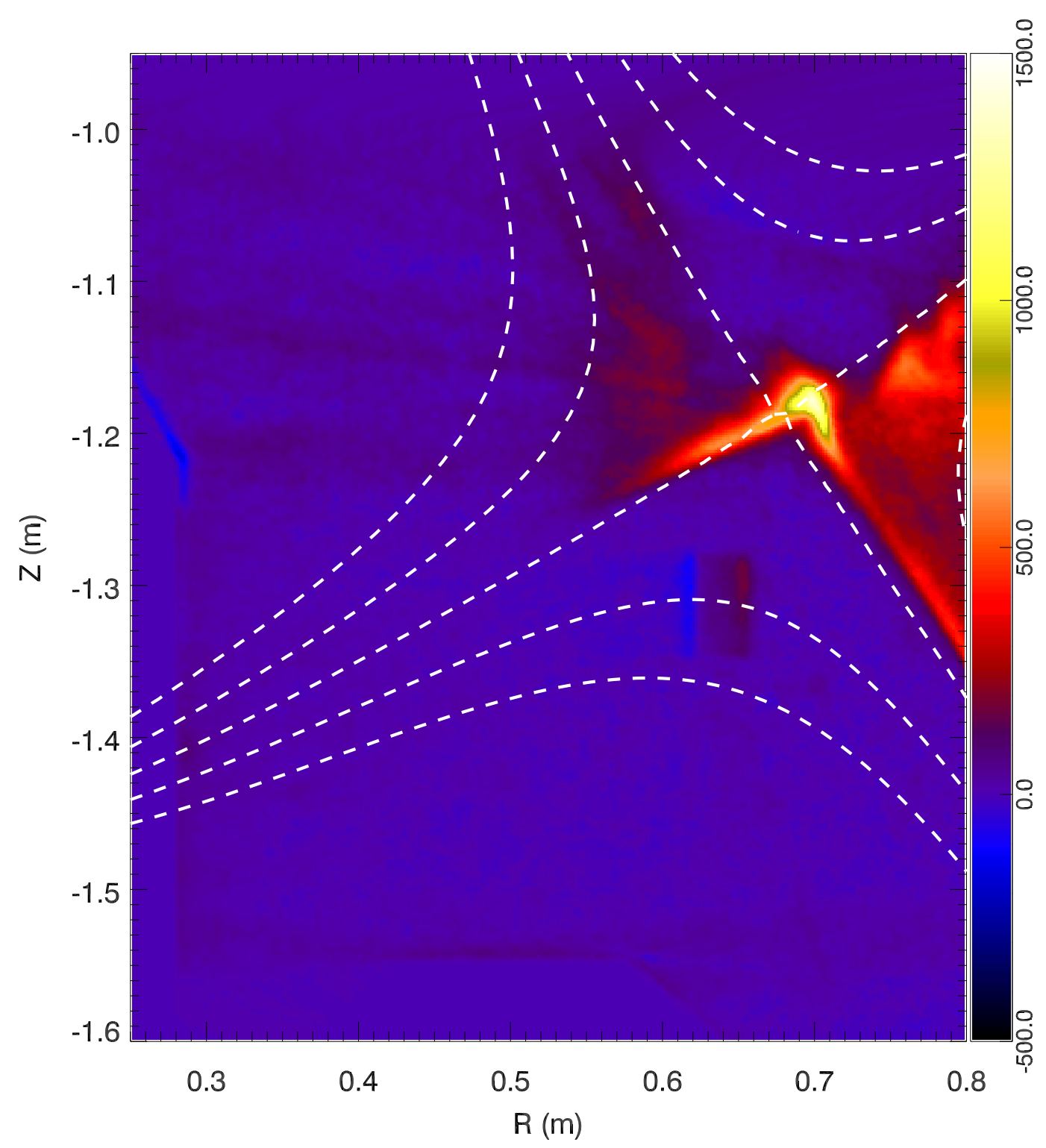

Figure 5.21: Tomographically inverted CIII (465nm) data from shot 25028 at $312 \mathrm{~ms}$, concentrating on the inner divertor leg with a magnetic equilibrium from EFIT. 


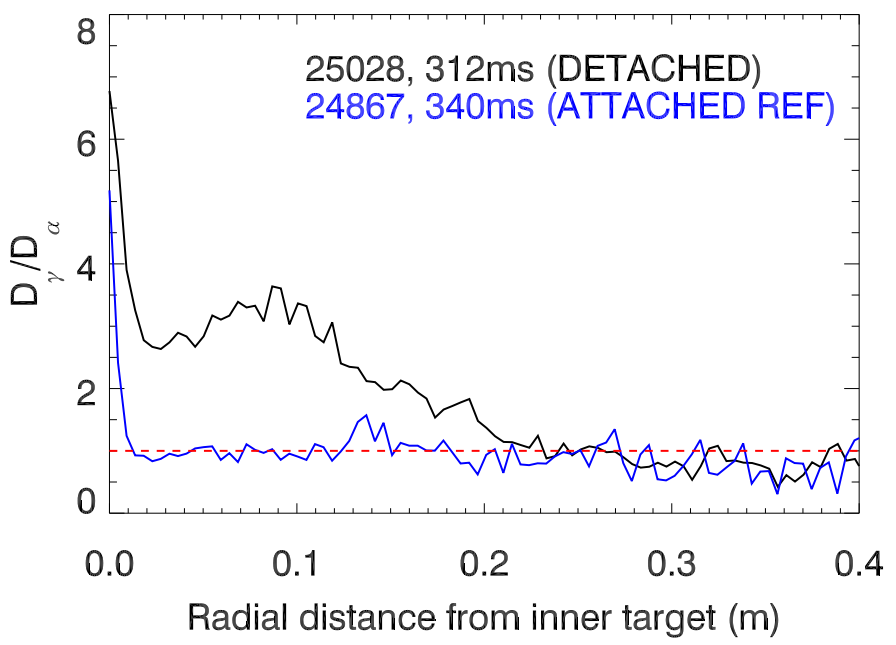

Figure 5.22: Ratio of $\mathrm{D}_{\gamma} / \mathrm{D}_{\alpha}$ line intensities during shot 25028 at $312 \mathrm{~ms}$ (black) and during an attached reference frame from shot 24867 at $340 \mathrm{~ms}$ (blue) as measured by DivCam. The data has been relatively calibrated such that $\mathrm{D}_{\gamma} / \mathrm{D}_{\alpha}=1$ in the divertor leg for attached conditions.
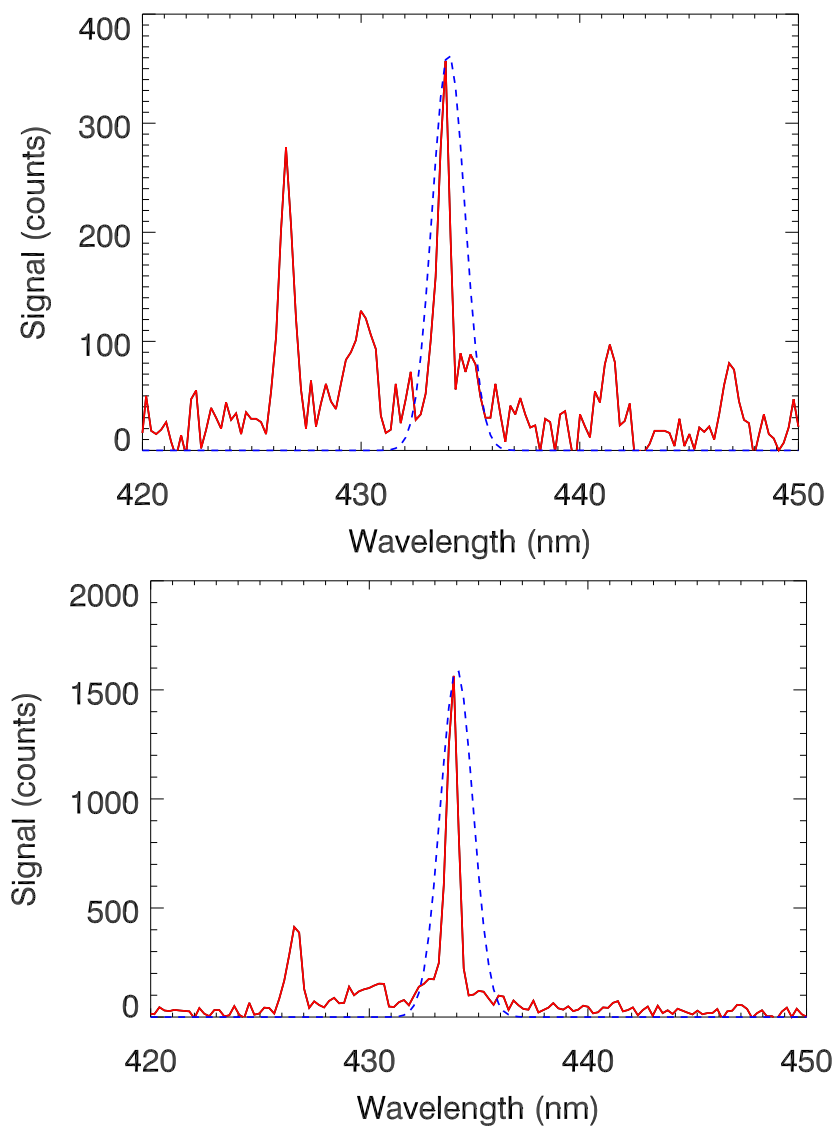

Figure 5.23: Survey spectra taken from the lower inner strike point from shot 24867 at $439 \mathrm{~ms}$ (top) and shot 25028 at $314 \mathrm{~ms}$ (bottom). The bandpass of the $\mathrm{D}_{\gamma}$ filter is indicated by the blue dashed curve. 


\subsection{Interpretive Detachment Modelling}

\subsubsection{OSM Setup}

In order to calculate an OSM plasma solution for shot 25028 at $312 \mathrm{~ms}$, ion flux data collected by the divertor Langmuir probes (figure 5.11) was input into the code. Small shifts in the data were applied to account for mis-alignment between the modelling grid and the ion flux data to ensure that the peak in the ion flux to the lower outer strike point corresponded with the location of the magnetic separatrix (at $\psi_{n}=1$ ). Upstream $n_{e}, T_{e}$ data was taken from the Thomson scattering diagnostic (figure 5.9) to constrain the plasma solution at the high-field side, closest to the detached lower inner divertor leg. As explained in section 5.3.1, the experimental data was manually filtered to ensure that perturbations to the measured data from sawtooth activity was removed by not using data within $\pm 1 \mathrm{~ms}$ of a sawtooth crash.

Spectroscopic measurements of line integrated $\mathrm{n}_{e}$ and line averaged $\mathrm{T}_{e}$ (figure 5.15) were used to constrain the OSM plasma solution in proximity of the lower inner divertor target. As outlined in section 5.3.5, the effects sawtooth activity could not be so easily removed from the data, as the integration times of the spectroscopic detectors used exceeds the sawtooth period. However, it was also noted that the effects of sawteeth should be mild, as the measured line emission will be predominantly from the recombining plasma. The analysed $n_{e}, T_{e}$ profiles from the raw data were interpolated to extract temperature and density profiles along the magnetic separatrix, and to facilitate mapping of the experimental data onto the OSM modelling grid. The use of line-integrated density and line-averaged temperature profiles neglects the effects of density and temperature variations along the spectroscopic lines of sight and possibly the implications of changing path length as the spatial extent of the recombining region changes due to sawtooth activity. Alignment of the lines of sight with the separatrix flux tube reduce the degree of varying plasma conditions along the line of sight, however.

Tomographically inverted spatial distribution of CII (514nm) and CIII (465nm) emission can be used to create approximate $\mathrm{T}_{e}$ contours using figure 5.16 and observations of the periphery of CII, CIII emission regions in figures $5.20 \& 5.21$. The $\mathrm{T}_{e}$ contours input into OSM in this study are shown in figure 5.24. The presence of features in the data due to mechanisms other than plasma line emission are highlighted. Their cause can be ascribed to photon reflections from stainless steel surfaces, such as mounting brackets for magnetic field coils, and the limited extent of the camera view relative to the inversion mesh. Alignment between the inverted data and EFIT equilibria is not perfect, however, due to reduced coverage of magnetic diagnostics in the vicinity of the lower inner strike point. Slight improvement in agreement between EFIT and the inverted data was found by imposing additional constraints from the 

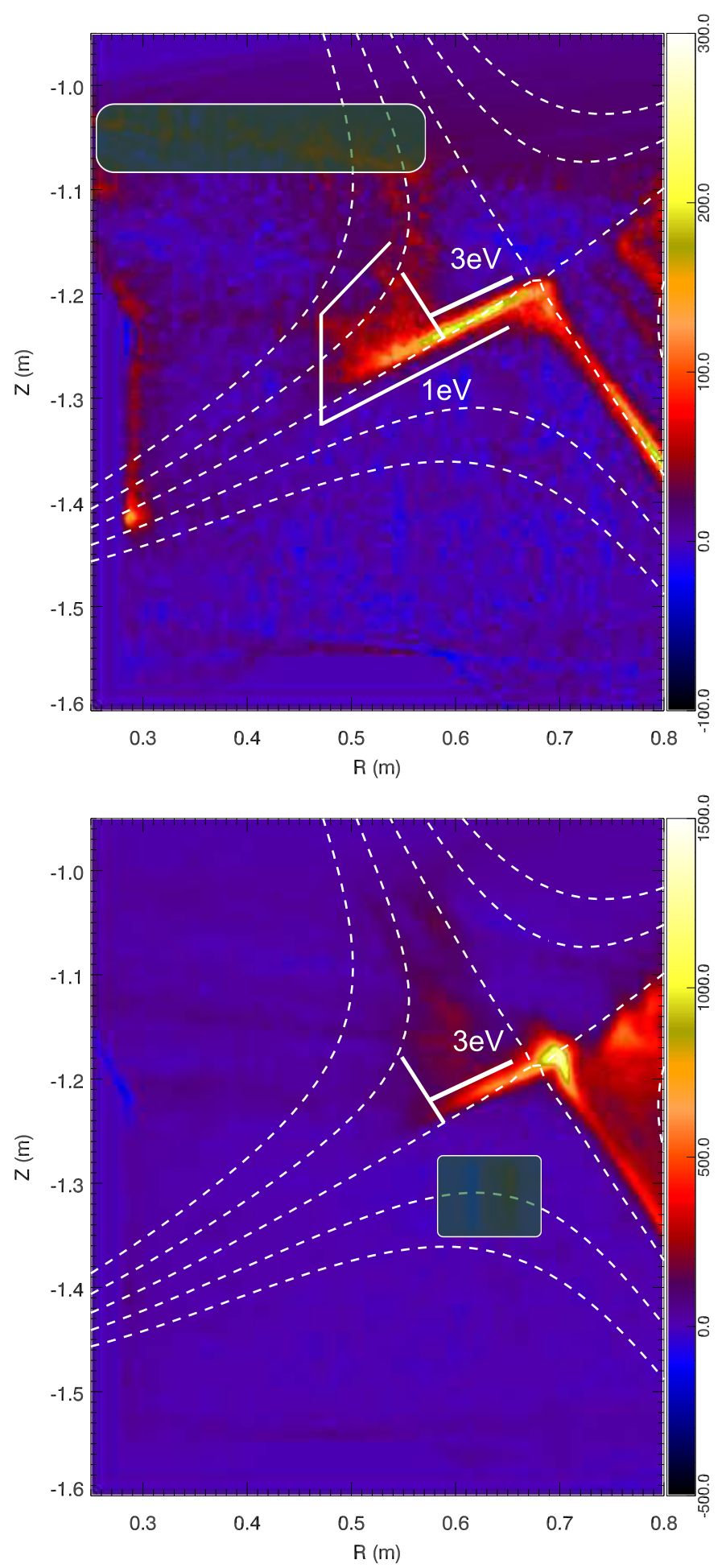

Figure 5.24: Approximate electron temperature profiles constructed using tomographically inverted CII $(514 \mathrm{~nm})$ and CIII $(465 \mathrm{~nm})$ emission data. The areas highlighted in green are most likely artefacts of the inversion process. The green area highlighted in the top figure is due to the inversion mesh being poorly covered by the camera view. The green area highlighted in the bottom figure is due to photon reflection from a P3 coil bracket. 
Thomson scattering diagnostic, which shifted the EFIT reconstruction by $\approx 1 \mathrm{~cm}$. The results of this reconstruction are plotted over the inverted data (figures 5.18-5.21).

Temperature contours formed using the periphery of the CII emission regions (corresponding to $\mathrm{T}_{e} \approx 1 \mathrm{eV}$ and $3 \mathrm{eV}$ at the low and high $\mathrm{T}_{e}$ sides of the $\mathrm{C}^{1+}$ abundance curve) are in agreement with the onset of the CIII emission region. These temperature contours are only approximate, and transport effects, which are not present in the ADAS calculations in figure 5.16, introduce additional uncertainties in the location of $\mathrm{T}_{e}$ contours. There is, however, disagreement between CIII emission and $\mathrm{T}_{e}$ measurements at the midplane from Thomson scattering, which indicate $\mathrm{T}_{e} \approx 5 \mathrm{eV}$ at the high-field side midplane. If this were the case, CIII emission should be visible in the vicinity of the high-field side midplane (figure 5.16), which is not supported by the data (figure 5.21). An explanation for this discrepancy is that the minimum detectable $\mathrm{T}_{e}$ measured by Thomson scattering is $\approx 5 \mathrm{eV}$ [113]. Therefore, it is possible that $\mathrm{T}_{e}<5 \mathrm{eV}$, in accordance with CIII emission data.

Inverted $\mathrm{D}_{\alpha}$ and $\mathrm{D}_{\gamma}$ emission are used in this study as a check on the quality of the OSM-EIRENE plasma solution by comparison of calculated $\mathrm{D}_{\alpha}$ and $\mathrm{D}_{\gamma}$ emissivities from EIRENE. As in the case of CII and CIII data, the inverted data contains artefacts from the inversion process, such as a horizontal line at $\mathrm{Z}=-1.4 \mathrm{~m}$ in figures 5.18 \& 5.19. Analysis of inverted $\mathrm{D}_{\gamma}(434 \mathrm{~nm})$ emission is complicated by the presence of as yet unidentified emission within the bandpass of the $\mathrm{D}_{\gamma}$ filter used in this study. There are some features in common between $\mathrm{D}_{\gamma}$ and CII inverted data, such as measured $\mathrm{D}_{\gamma}$ emission at $\mathrm{Z}=-1.43, \mathrm{R}=0.29$ close to the strike point below the separatrix, perhaps suggesting that this emission has a component arising from CII species.

\subsubsection{Results}

The data summarised in this chapter was input into OSM and interpolated onto the magnetic grid (figure 5.25) in order to constrain the plasma solver as much as possible, and to verify the plasma solution (and, by extension, its constraints) by comparing simulated $\mathrm{D}_{\gamma} / \mathrm{D}_{\alpha}$ emissivity line ratios with experimental observations. There are, however, still numerous free parameters not constrained by the code, principally the parallel ion velocity and the ion temperature (pressure) along the divertor leg. $\mathrm{T}_{i}=\mathrm{T}_{e}$ has been imposed upstream and at the divertor targets. The numerical solver here is the original numerical scheme, as solver extensions such as cross-field drift motion, significantly hinder solver convergence in the recombining region. Particle sources and sinks due to ionisation and recombination reactions respectively calculated from EIRENE are included in the particle balance equation. Momentum sinks due to ionneutral friction were not included in the momentum balance equation in order to ascertain the properties of the momentum fitting source using EIRENE ionisation and 


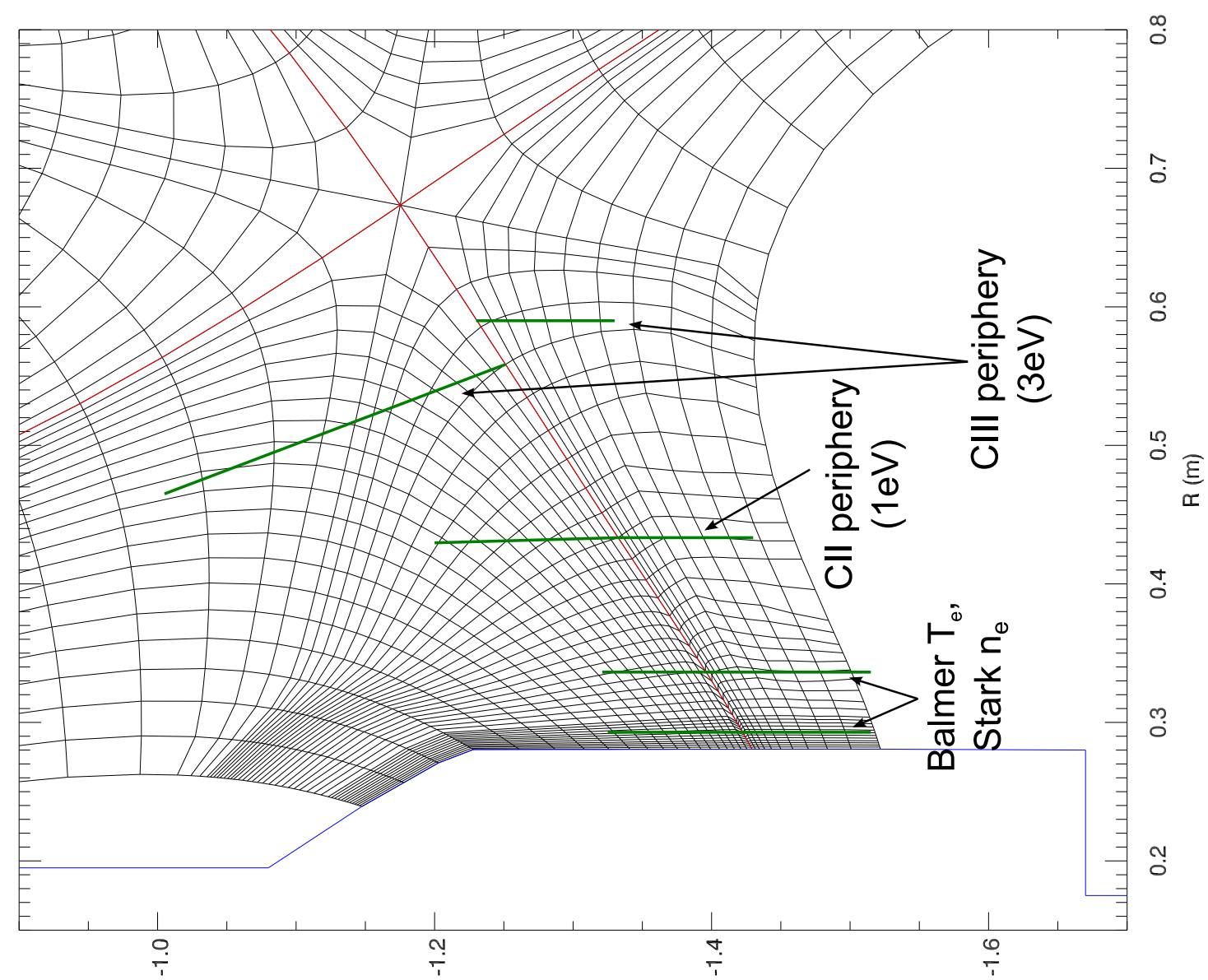

(w) Z

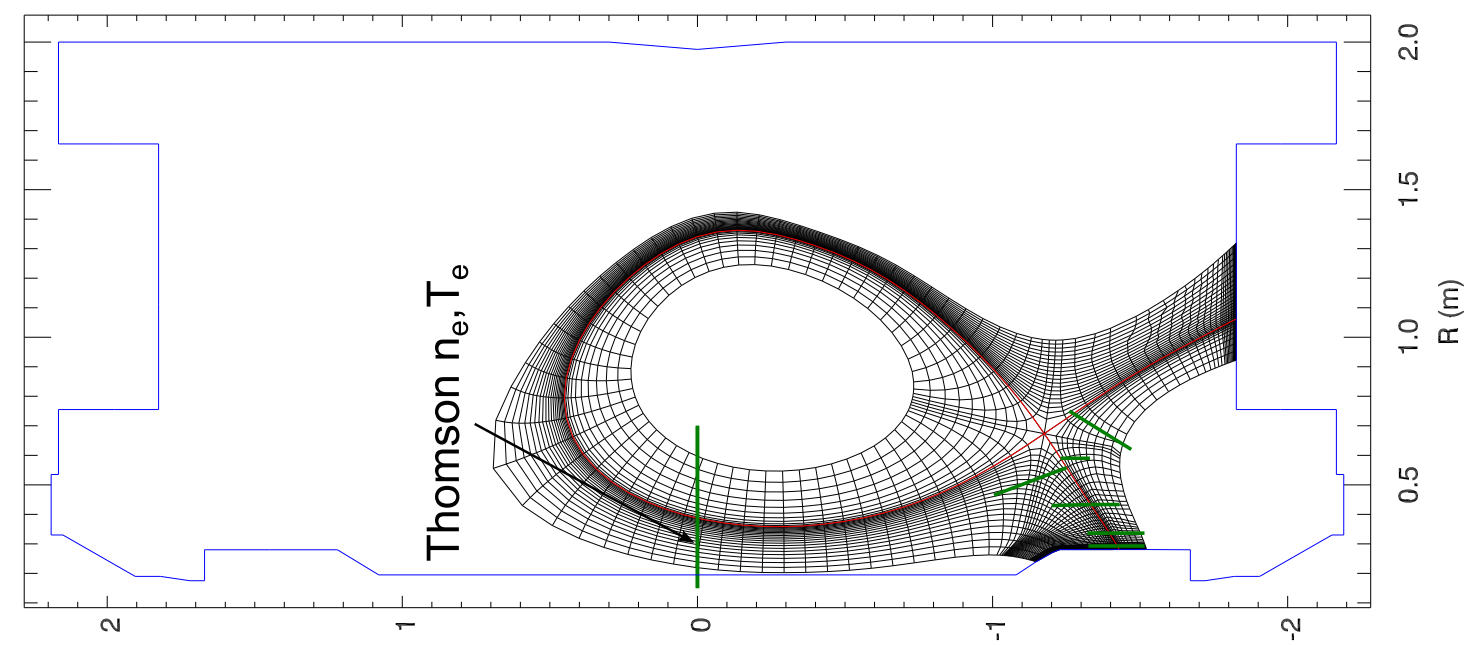

(w) Z

Figure 5.25: Magnetic grid used to simulate MAST shot 25028 at $312 \mathrm{~ms}$. The green lines indicate where experimental data has been used to constrain the OSM plasma solution, with the exception of ion flux data as measured by Langmuir probes embedded in the divertor targets. 
recombination particle sources and sinks. It is left for future work to investigate the role of the EIRENE momentum sink on the plasma solution.

The converged OSM-EIRENE plasma solution for the lower inner divertor leg is shown in figures $5.26 \& 5.27$, with the EIRENE calculated $\mathrm{D}_{\alpha}$ and $\mathrm{D}_{\gamma}$ emissivities shown in figure 5.28 due to atomic and molecular processes outlined in table 5.2. The plasma solution close to the lower inner strike point (figure 5.26) is heavily constrained to follow $n_{e}$ profiles specified by Stark broadening measurements, and $T_{e}$ profiles specified by high-n Balmer line ratio measurements (figure 5.15), CII (514nm figure 5.20) and CIII (465nm, 5.21) emission contours. The detached divertor target manifests as a strong particle recombination sink. Convergence to experimentally determined electron static pressures in this region involves invoking a very strong fitting momentum source in close proximity to the target plate, which subsequently decreases to bring about a pressure drop with increasing distance from the strike point specified by experimental data.

The simulated EIRENE Balmer emission is in good qualitative agreement with the inverted DivCam data in terms of the shape and spatial extent of the emission peak at the strike point. There is, however, a strong $\mathrm{D}_{\alpha}$ and $\mathrm{D}_{\gamma}$ emission region due to the $\mathrm{D}_{2}$ gas puff at the top of the vertical target, which is not seen in the inverted camera data. There is a region of elevated $\mathrm{D}_{\gamma}$ emission in this vicinity in figure 5.19 , however, there is also CII emission in this region (figure 5.20), suggesting this signal could be due to carbon contamination. Analysis of the processes that give rise to this emission (figure 5.31) indicate a strong molecular contribution. Simulations have shown that the presence of the gas puff is unchanged if $\mathrm{T}_{e}$ is varied from $1 \mathrm{eV}$ to $5 \mathrm{eV}$ close to the gas puff, suggesting that the discrepancy is not due to inaccuracies in the plasma solution. Another possibility, therefore, that the molecular densities calculated by EIRENE are not an accurate reflection of the experimental conditions. Benchmarking of the EIRENE simulation, other than the manner described, in the vicinity of the inner strike point is difficult as other calculated quantities such as atomic and molecular densities are not measured in this region on MAST.

Comparing measured with simulated $\mathrm{D}_{\gamma} / \mathrm{D}_{\alpha}$ line emission ratios along the separatrix in the vicinity of the lower inner strike point (figure 5.29) shows good agreement in the spatial extent of the recombining region, indicated by $\mathrm{D}_{\gamma} / \mathrm{D}_{\alpha}>1$. As with the inverted camera data, the EIRENE Balmer emissivities were calibrated such that $\mathrm{D}_{\gamma} / \mathrm{D}_{\alpha}=1$ in attached conditions, calculating using emissivities calculated close to the $\mathrm{x}$-point, which showed no signs of volume recombination. Within the recombining region, the level of agreement is within a factor of 2 . This is an encouraging indication that the OSM plasma solution is an accurate representation of the plasma under examination. This result is also in agreement with interpretive modelling efforts at 
Alcator C-Mod [114], which revealed discrepancies between EIRENE calculated and observed $\mathrm{D}_{\gamma}$ emissision by a factor of $\approx 2$, although in that case, absolute line intensities were being directly compared.

It is unlikely that the discrepancy has the same cause as the appearance of the $\mathrm{D}_{2}$ gas puff in the EIRENE calculations. The dominant mechanism for the creation of Balmer photons is recombination in the detached region (figure 5.30), in contrast to molecular dissociation processes close to the gas puff (figure 5.31). The influence of experimental uncertainties in the experimental data used to constrain the plasma solution and their relation to uncertainties in calculated Balmer emissivities remains unresolved and will be a subject of future work on this subject.

Close to the divertor target, there is a strong divergence between the calculated and simulated line ratios. The source of this discrepancy may be due to contamination of the observed spectral emission lines due to spectral emission other than $\mathrm{D}_{\gamma}$ within the bandpass of the filter used. This hypothesis is supported by $\mathrm{D}_{\gamma} / \mathrm{D}_{\alpha}$ measurements taken as an attached reference for relative calibration purposes (figure 5.22), where the emission ratio increases significantly above 1 in close proximity to the divertor strike point.

\begin{tabular}{|c|c|c|}
\hline Index & Reaction & Notes \\
\hline 1 & $\mathrm{e}+\mathrm{H}^{+} \rightarrow \mathrm{H}+\gamma$ & Radiative volume recombination \\
\hline 2 & $\mathrm{e}+\mathrm{e}+\mathrm{H}^{+} \rightarrow \mathrm{e}+\mathrm{H}$ & 3-body volume recombination \\
\hline 3 & $\mathrm{e}+\mathrm{H} \rightarrow 2 \mathrm{e}+\mathrm{H}^{+}$ & Atomic ionisation \\
\hline 4 & $\mathrm{H}+\mathrm{H}^{+} \rightarrow \mathrm{H}+\mathrm{H}^{+}$ & $\begin{array}{c}\text { Elastic collisions between atoms } \\
\text { (including charge-exchange) }\end{array}$ \\
\hline 5 & $\mathrm{e}+\mathrm{H}_{2} \rightarrow \mathrm{e}+\mathrm{H}+\mathrm{H}$ & Dissociation \\
\hline 6 & $\mathrm{e}+\mathrm{H}_{2} \rightarrow 2 \mathrm{e}+\mathrm{H}+\mathrm{H}^{+}$ & Molecular ionisation \\
\hline 7 & $\mathrm{e}+\mathrm{H}_{2} \rightarrow 2 \mathrm{e}+\mathrm{H}_{2}^{+}$ & Ion conversion \\
\hline 8 & $\mathrm{H}^{+}+\mathrm{H}_{2}(\nu) \rightarrow \mathrm{H}+\mathrm{H}^{+}$ & Dissociative recombination (MAR with reaction 8 ) \\
\hline 9 & $\mathrm{e}+\mathrm{H}_{2}^{+} \rightarrow \mathrm{H}+\mathrm{H}$ & Dissociation (MAD with reaction 8) \\
\hline 10 & $\mathrm{e}+\mathrm{H}_{2}^{+} \rightarrow \mathrm{e}+\mathrm{H}+\mathrm{H}^{+}$ & Dissociative ionisation (MAI with reaction 8) \\
\hline 11 & $\mathrm{e}+\mathrm{H}_{2}^{+} \rightarrow 2 \mathrm{e}+\mathrm{H}^{+}+\mathrm{H}^{+}$ & \\
\hline
\end{tabular}

Table 5.2: Neutral collision processes included in EIRENE as set up for this study [115].

\subsection{Summary}

An extensive experimental dataset has been collected to characterise the plasma conditions within a partially detached discharge on MAST. These include measurements of upstream $n_{e}, T_{e}$ upstream of the recombining region with unprecedented $(\approx 1 \mathrm{~cm})$ spatial resolution with a newly upgraded Thomson scattering diagnostic. Ion flux to the divertor target plate was measured using embedded Langmuir probes with 


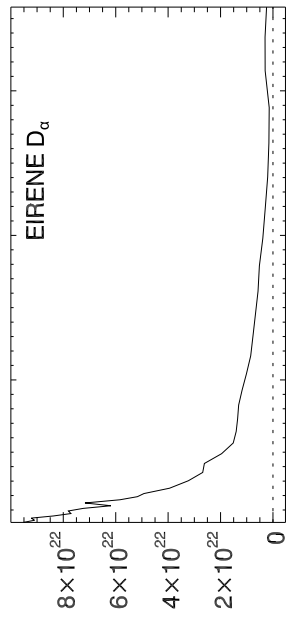

$\left({ }_{1-s_{\varepsilon}-} m u d\right)$

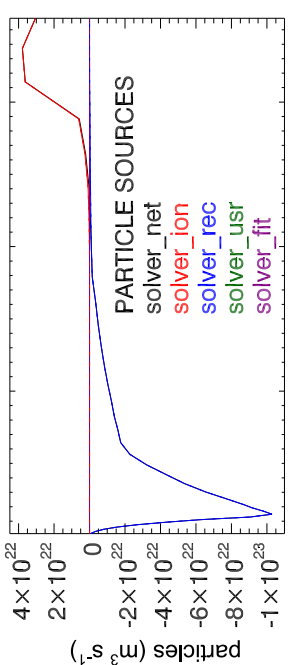

$\left({ }_{L}-s_{\varepsilon} w\right)$ sə|ग!̣ued

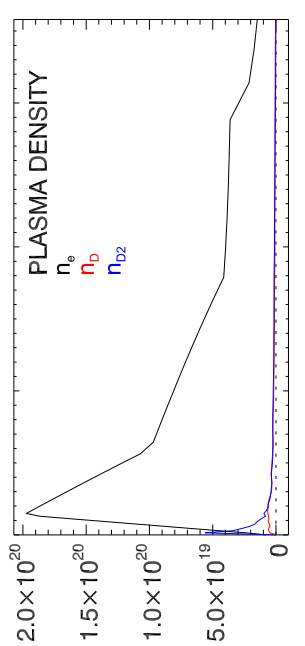

$\left(\varepsilon_{\varepsilon}-u\right) !$
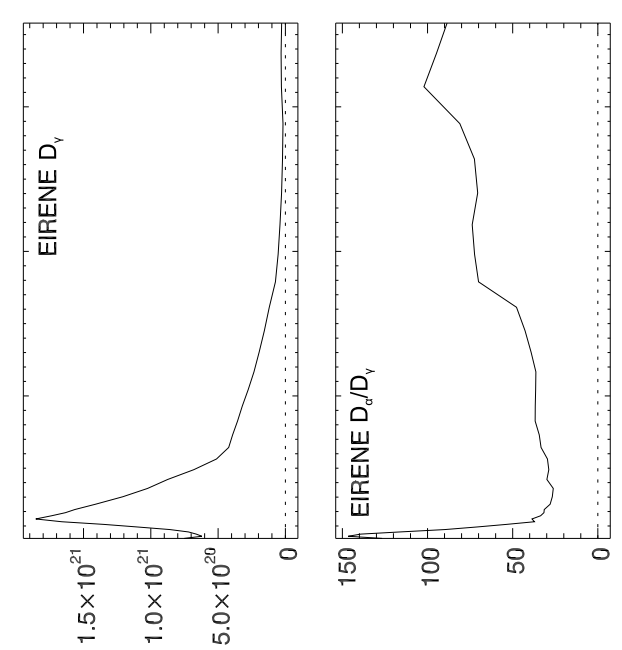

(..s. $\varepsilon_{\varepsilon} \cdot \mathrm{W}$ yd)
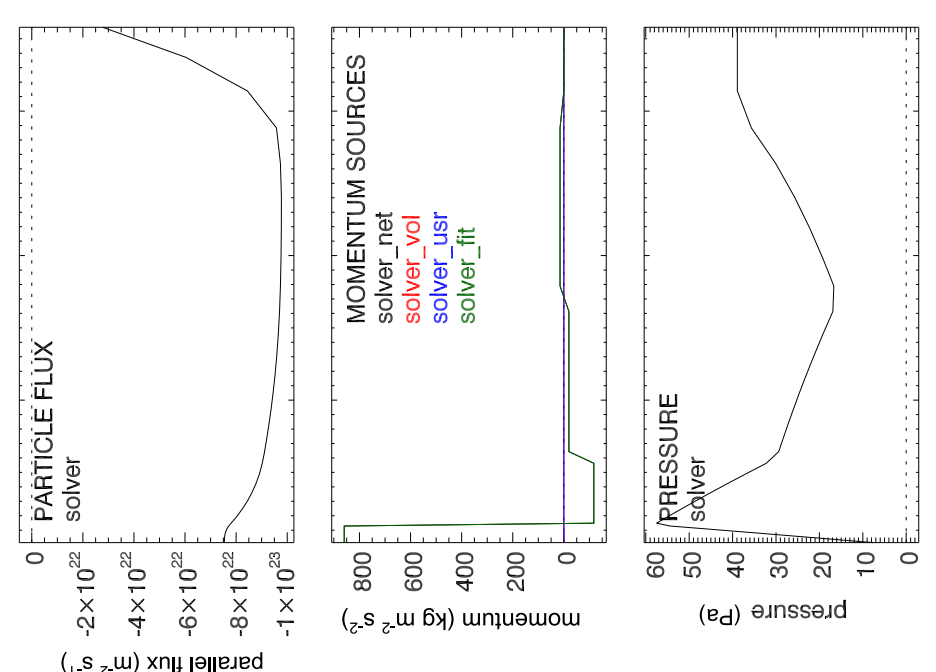

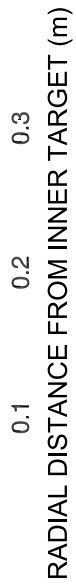

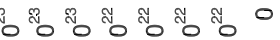

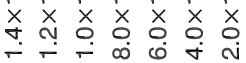

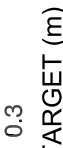

采

ำ

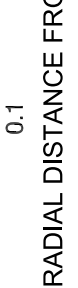

.



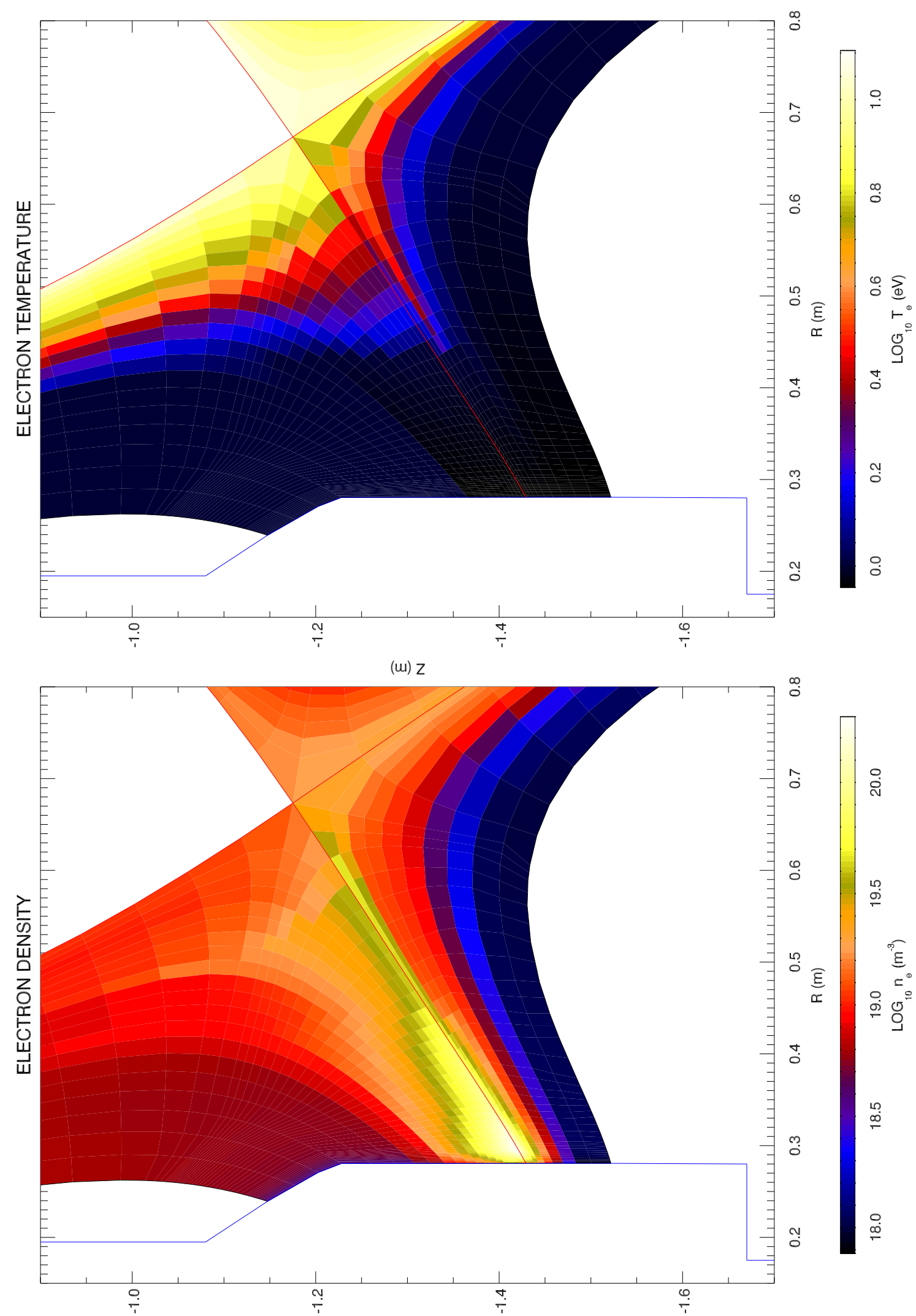

(w) Z

Figure 5.27: OSM plasma solution for shot 25028 at $312 \mathrm{~ms}$. The electron temperature close to the lower inner divertor leg is low $(\leq 1 \mathrm{eV})$, whereas the electron density increases in this region. 

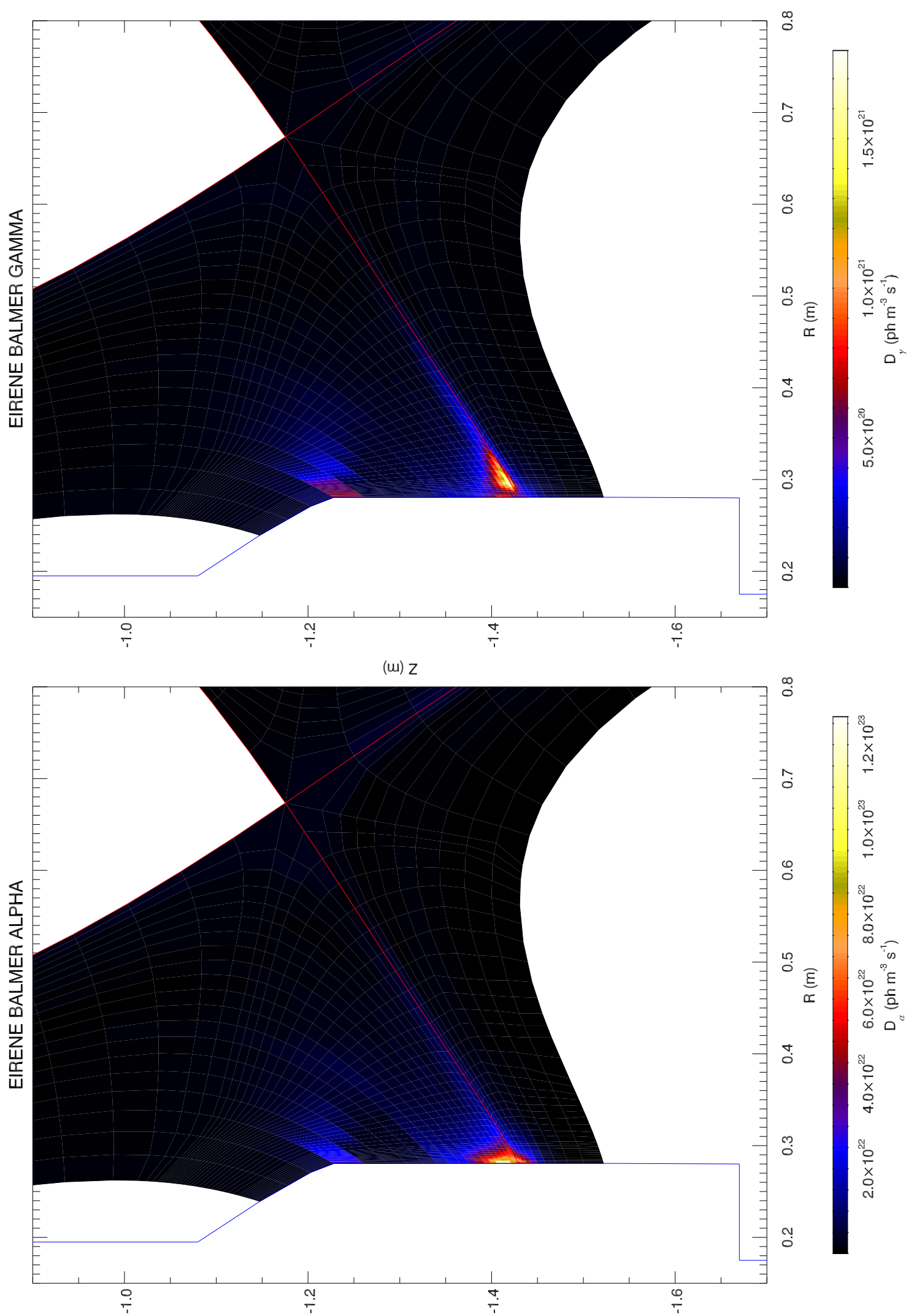

(w) Z

Figure 5.28: OSM-ERIENE calculated $\mathrm{D}_{\alpha}$ and $\mathrm{D}_{\gamma}$ emissivities. The $\mathrm{D}_{2}$ gas puff is clearly visible in both emission lines, contrary to experimental observations. 


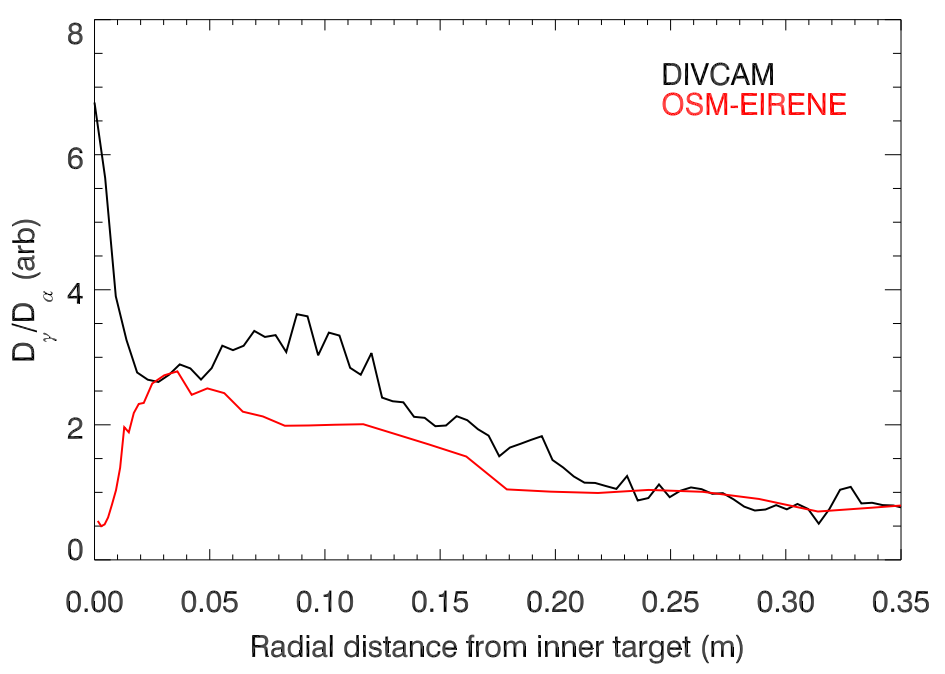

Figure 5.29: Comparison of tomographically inverted $\mathrm{D}_{\gamma} / \mathrm{D}_{\alpha}$ emission measured by DivCam and an OSM-EIRENE simulation.
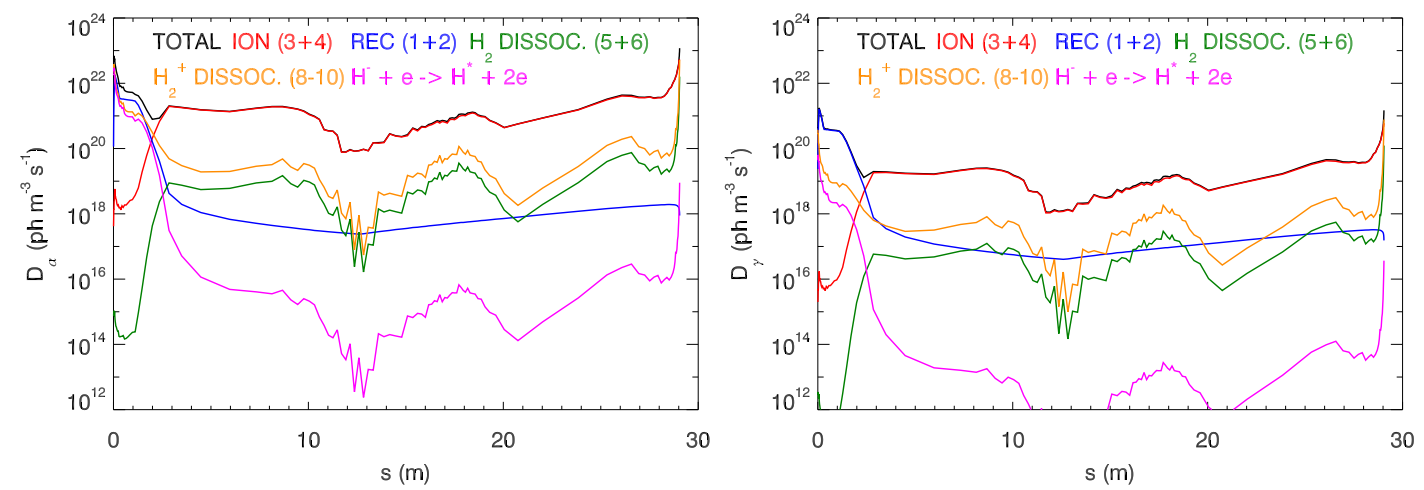

Figure 5.30: Breakdown of $\mathrm{D}_{\alpha}$ (left) and $\mathrm{D}_{\gamma}$ (right) emission in terms of atomic and molecular physics processes (table 5.2) along the separatrix flux tube, calculated by EIRENE.
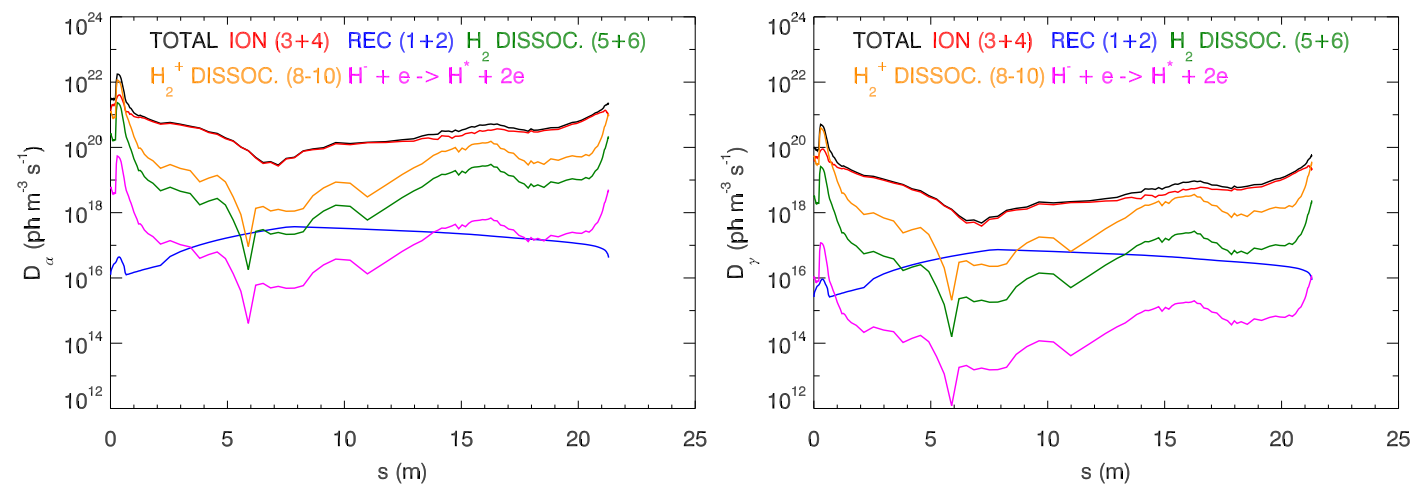

Figure 5.31: Breakdown of $\mathrm{D}_{\alpha}$ (left) and $\mathrm{D}_{\gamma}$ (right) emission in terms of atomic and molecular processes along a flux tube intersecting the lower inner $\mathrm{D}_{2}$ gas puff (located at $\mathrm{s} \approx 0.3 \mathrm{~m})$, calculated by EIRENE. 
$6 \mathrm{~mm}-9 \mathrm{~mm}$ spatial resolution. Line integrated $n_{e}$ and line-averaged $T_{e}$ along the lower inner divertor leg with a spatial resolution of $7 \mathrm{~mm}$ were measured using a line-ofsight spectroscopy diagnostic developed during this study. Imaging spectroscopy of CII (514nm), CIII (465nm), $\mathrm{D}_{\alpha}(656 \mathrm{~nm})$ and $\mathrm{D}_{\gamma}(434 \mathrm{~nm})$ emission lines were carried out and tomographically inverted using a damped conjugate-gradient method [60].

The OSM-ERIENE code was initially used to check the experimental data for signs of internal consistency for data originating from the different diagnostics. It has been found that the data collected was broadly consistent, within uncertainties and the capabilities of the diagnostics used. The plasma solution generated was directly compared with measurements of the ratio of $\mathrm{D}_{\gamma}$ and $\mathrm{D}_{\alpha}$ line emissivities, and agreement was found within a factor of 2 within the recombining region. The deviation of measured and simulated $\mathrm{D}_{\gamma}$ and $\mathrm{D}_{\alpha}$ line emission close to the divertor target is likely due to CII line radiation within the bandpass of the $\mathrm{D}_{\gamma}$ filter used. 


\section{Chapter 6}

\section{Conclusions and Future Work}

\subsubsection{Conclusions}

The work presented in this thesis represents efforts to better understand particle and momentum transport in the MAST Tokamak in in attached and detached divertor conditions using the interpretive OSM-EIRENE code. To assist these modelling efforts in detached plasmas, experimental data from a large number of diagnostics were used to act as constraints and boundary conditions for the code. The nature of detached plasmas (low $\mathrm{T}_{e}$, high $\mathrm{n}_{e}$ ) necessitated the modification and development of spectroscopic diagnostics and analysis codes that are capable of producing profiles of line integrated electron density and line averaged electron temperature along magnetic flux surfaces.

The interpretive OSM-EIRENE code was first applied to the problem of explaining a drop in total plasma pressure routinely observed between the outer midplane and lower outer divertor in attached conditions. The undertaking of this study necessitated the implementation of new numerical solvers in order to solve more complex conservation laws as different aspects of plasma transport were invoked to address the problem. It was found that magnetic flux expansion terms do not address the pressure drop directly, but significantly reduce, by a factor of 5, "fitting" sources required for the OSM plasma solution to coincide with experimental observations. This suggests that the inclusion of these additional transport terms represent a more exact description of parallel transport in the MAST SOL.

Parallel dynamic viscosity was introduced into the OSM equations, which resulted in a small increase in the pressure drop between the midplane and the divertor overall, but also reduced the magnitude of fitting source terms by a factor of 2 . The use of kinetic limits on the viscosity model used was investigated, finding that the plasma conditions in MAST are such that the standard description of parallel viscosity can be invoked in this case.

The effects of cross-field drift motion on the pressure discrepancy was also explored. 
This required the formulation of a stable numerical method capable of solving the combined Ohm's law and current conservation equations to high accuracy. Closer examination of the conservation equations revealed that flux expansion terms had a strong effect on parallel plasma potential profiles in the region where $\nabla_{\|} \mathrm{B} / \mathrm{B}$ was large. Invoking diamagnetic drift terms led to a reduction in the observed pressure drop and a reduction in solver fitting sources by $20 \%$. Conversely, introduction of the $\vec{E} \times \vec{B}$ drift resulted in an increased pressure drop and an increase in solver fitting sources by a factor of 5 . It was also found OSM-EIRENE calculations involving $\vec{E} \times \vec{B}$ sources were very sensitive to small shifts in the experimental data used as boundary conditions for the code.

The effects of varying the ion temperature with respect to the electron temperature were also investigated, varying $T_{i} / T_{e}$ from 1 to 3 . It was found that over this range, solver fitting sources were reduced by factors of 1 to 1.5 respectively. It was also found that some values of $T_{i} / T_{e}$ were found to explain the pressure drop, assuming this ratio varied poloidally.

Experiments were led to achieve the detached divertor state on MAST for the first time since the installation of a new divertor in 2004. The experiments were successful at low neutral beam heating power $(\approx 1 \mathrm{MW})$. To ascertain the plasma conditions in the detached lower inner divertor leg, a multi-chord imaging spectrometer was deployed with new input optics designed to increase spectral performance and reduce cross-talk between channels. Data analysis codes were written to ascertain the line averaged electron temperature within the detached divertor leg using the intensity of high-n Balmer emission lines. Measurements of line-integrated electron density were made using measurements of the widths of Stark broadened high-n Balmer emission lines, and a new analysis code based on theoretical calculations of Stark broadened spectral line shapes at Tokamak-relevant densities. With these advancements, parallel electron static pressure profiles were measured along the separatrix field line during the detached phase of a MAST discharge with high $(\approx 7 \mathrm{~mm})$ spatial resolution. This data was combined with upstream $n_{e}, T_{e}$ data recorded upstream of the recombining region provided by a recently upgraded Thomson scattering diagnostic, with $1 \mathrm{~cm}$ radial resolution. Ion fluxes to the divertor target plates were measured with high $(6-9 \mathrm{~mm})$ radial resolution using Langmuir probes embedded in the divertor target plates. The Langmuir probe data revealed a localised reduction in the target ion flux, which has not been reported on other diverted Tokamaks. Narrow-bandwidth imaging of $\mathrm{D}_{\alpha}, \mathrm{D}_{\gamma}$, CII and CIII spectral lines at high spatial $(\approx 3 \mathrm{~mm})$ and temporal $(5 \mathrm{kHz})$ resolution were recorded and tomographically inverted with a spatial resolution of $\approx 5 \mathrm{~mm}$.

Tomographically inverted CII and CIII data were used to impose constraints on $\mathrm{T}_{e}$ at the edge of the emission regions, using atomic data from the ADAS codes. The 
analysed experimental data was input into the OSM-EIRENE code to simulate $\mathrm{D}_{\gamma}$ and $\mathrm{D}_{\alpha}$ emissivities in the recombining region. The results of the simulation were checked against experimental observations to verify the quality of the OSM plasma reconstruction. The calculated and inverted $\mathrm{D}_{\gamma} / \mathrm{D}_{\alpha}$ line ratios agreed within a factor of 2 across the recombining region, indicating the OSM plasma solution is a reasonably accurate representation of the plasma under investigation. The visibility of a $\mathrm{D}_{2}$ gas puff in EIRENE simulations not supported by experimental observations. Methods for resolving this discrepancy will be the subject of future investigations.

\subsubsection{Future Work}

The work presented here is an example of how an interpretive modelling code can be used to investigate the effects of different transport terms on attached plasmas, and to re-reconstruct the plasma conditions within detached plasmas, and assess the accuracy of that reconstruction. A clear avenue of future investigation is to broaden the scope of this study to investigate the effects of plasma transport terms on detached plasma reconstructions and their agreement, or otherwise, with experimental observations. Such work may elucidate the physics governing detachment and it's onset, which remains an important open question that must be addressed to ensure the longevity of ITER and its successors.

In terms of future experimental work, the validation of the He line ratio technique [60] by applying the method to detached plasmas and comparison with results from techniques outlined in this thesis could yield new insights into the formation and conditions within detached plasmas with unprecedented detail. Expanding the diagnostic dataset to include HeII, C-D and D-D emission could further constrain the model used here, and perhaps offer insight into the discrepancy between OSM-EIRENE simulations and observations presented here. Attempting divertor detachment in $\mathrm{H}-$ mode discharges, such as studies carried out on ASDEX-Upgrade [116] could also yield new insight. Data from additional diagnostics, such as ion temperature measurements using Retarding Field Energy Analysers or otherwise could address a key uncertainty in the simulations of detached plasmas in general. Furthermore, measurement of the ion flow field using Mach probes or Doppler spectroscopy in the entrance to the recombining region could help resolve another key uncertainty in our knowledge of detached plasmas.

Future modelling studies will focus on the role of impurities (principally carbon) in detachment, by introducing additional species in the OSM transport equations in order to assess their role in particle, momentum and power balance. The OSM numerical scheme will be further refined to include a heat transport model for both ion and electron species, in order to gain better insight into the heat transport mechanisms during detachment and in the hope of resolving the currently open question concerning the 
ion temperature profile in the plasma under investigation. Incorporation of momentum loss terms due to ion-neutral friction from the EIRENE code would also be a valuable addition to the modelling efforts presented here. 


\section{Appendix A}

\section{OSM Solver Development}

This appendix describes the numerical enhancements that have been made to OSM during this study. New means of solving particle and momentum conservation equations give the OSM code additional stability and the ability to extend the conservation equations used. A new numerical code has been developed to calculate accurate solutions to the combined Ohm's law and current conservation equations in a highly stable manner.

\section{A.1 The Runge-Kutta Method}

The original OSM numerical scheme as outlined in section 4.1 was designed specifically to solve the particle and momentum conservation equations by integrating the respective particle and momentum sources and solving the equations algebraically. This method, although simple to implement and extremely fast, would sometimes calculate complex solutions tot the conservation equations and hindered the implementation of more advanced transport equations. To remedy the latter drawback, a numerical solver based on the Runge-Kutta method [117] was implemented to handle more complex conservation laws. Runge-Kutta refers to a family of methods which originate from the simpler Euler method, which states:

$$
y_{n+1}=y_{n}+h f\left(x_{n}, y_{n}\right)
$$

where $y_{n}$ is a quantity at some given spatial (or temporal) step, $h$ is the step size, $f\left(y_{n}\right)$ is the spatial (or temporal) gradient of the quantity, calculated at point $y_{n}$ and $y_{n+1}$ is the quantity at the next step. The Euler method divides an integration domain into a number of steps, of size $h$, and the solution is advanced, one step at a time, over the integration domain. This method is first order accuracy, as stepping from one location to the next involves retaining the first two terms in a taylor series expansion of $y$ about step $n$. This means that the error in the estimate of $y_{n}$ is of the order of the 


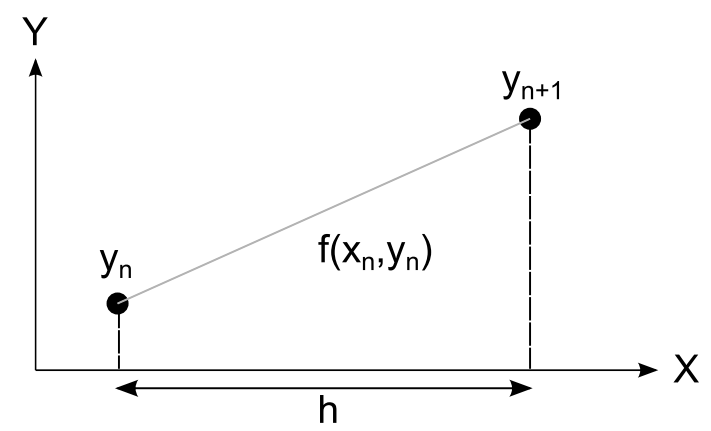

Figure A.1: Principle of the Euler method, $y_{n+1}=y_{n}+h f\left(x_{n}, y_{n}\right)$

square of the stepsize, $h^{2}$. To increase the accuracy of this method, higher-order terms in the Taylor series expansion could be preserved in the calculation of $y_{n+1}$, which is the reasoning behind the Runge-Kutta methods.

The method used in this study is a $5^{\text {th }}$ order algorithm based on the Cash-Karp Runge-Kutta method described in [67]. As part of the algorithm, the step size is automatically adjusted such that the accuracy of the solution, compared with one produced by a $4^{\text {th }}$ order method meets a pre-set requirement set by the user. In order to implement a Runge-Kutta solver with OSM, the particle and momentum conservation equations must be re-cast in the form of gradients in density and parallel ion velocity:

$$
\begin{gathered}
\frac{d n}{d s}=\frac{n\left(n \frac{d\left(T_{e}+T_{i}\right)}{d s}-S_{M}\right)+S_{P}\left(2 m_{e} n_{e} u_{\|}\right)}{u_{\|}\left(2 m_{e} n_{e} u_{\|}\right)-n\left(T_{e}+T_{i}+m_{e} u_{\|}\right)} \\
\frac{d u_{\|}}{d s}=\frac{-S_{P}\left(T_{e}+T_{i}+m_{e} u_{\|}\right)-u_{\|}\left(n \frac{d\left(T_{e}+T_{i}\right)}{d s}-S_{M}\right)}{u_{\|}\left(2 m_{e} n_{e} u_{\|}\right)-n\left(T_{e}+T_{i}+m_{e} u_{\|}\right)}
\end{gathered}
$$

Boundary conditions are provided by specifying values for density and parallel ion velocity at the start of the integration domain. In order to check for consistency, the results provided by this method were checked with those provided by the original algorithm for the primary separatrix flux tube for MAST shot 24861 at $240 \mathrm{~ms}$ as defined in chapter 4 (figure A.2). The two numerical schemes give similar converged solutions when the same original OSM particle and momentum conservation equations are input used in the Runge-Kutta solver.

\section{A.2 The Finite-Volume Method}

The finite-volume method [118] is a means of discretising partial differential equations to reduce them to a system of algebraic equations, like the Runge-Kutta method, except the details of how the equations are formed is altered. Figure A.3 outlines 

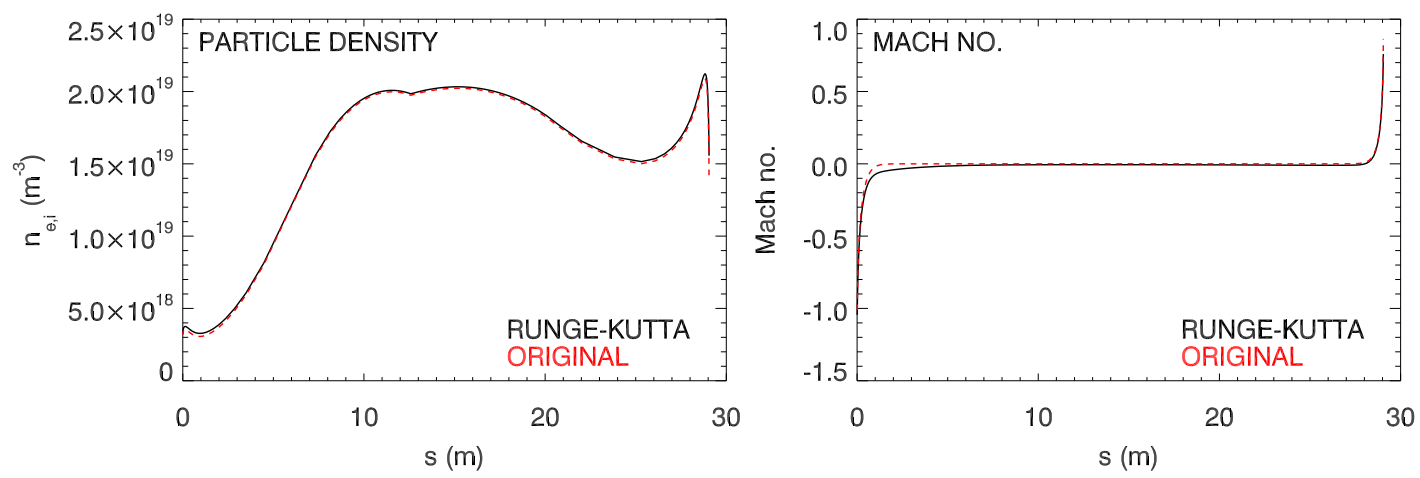

Figure A.2: Comparison of OSM solutions calculated with the original and RungeKutta algorithms with the same geometry and boundary conditions.

how space is discretised in the finite-volume method. As opposed to the Runge-Kutta method earlier, which is an example of a method based upon finite differences, the finite-volume method consists of solving conservation equations within cells distributed to fill the simulation domain.

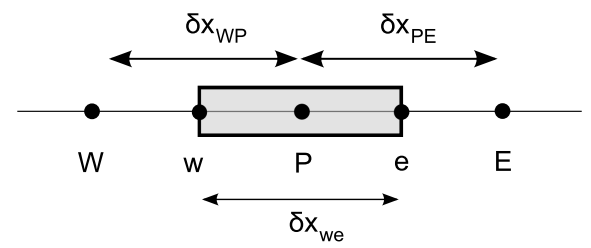

Figure A.3: Geometry used in the finite-volume discetisation. Uppercase letters indicate computational cells, lowercase letters indicate cell faces.

As opposed to the finite difference method, where quantities of interest are calculated at nodes and conservation equations dictate how the solution evolves from one node to the next, the finite volume method requires the segregation of the simulation domain into a series of cells. At the centre of each cell, the quantity of interest is calculated, by relating the fluxes of a quantity entering and leaving cell faces, and the source/sink rate of that quantity. Such a method is well-suited to solving flow problems in fluid dynamics, where it is applied frequently. This form of spatial discretisation also exists in OSM, and so finite-volume methods are well suited for this application.

To calculate the value of a given conserved quantity at cell $\mathrm{P}$ (figure A.3), the methods employed in this study use information from cells connected to the east (e) and west $(\mathrm{w})$ faces of the cell. Precisely how the algebraic equations are formed is now described in the stationary (time-independent) case.

Consider a convection-diffusion equation:

$$
\frac{\partial(\rho \phi)}{\partial x}=\frac{\partial}{\partial x}\left(\Gamma \frac{\partial \phi}{\partial x}\right)+S
$$


by integrating over the control volume $V$ and using the divergence theorem:

$$
\int_{V} \nabla \cdot \vec{A} d V=\int_{A} \vec{A} \cdot \hat{n} d A
$$

gives

$$
\int_{A} \rho \phi d A=\int_{A}\left(\Gamma \frac{\partial \phi}{\partial x}\right) d A+\int_{V} S d V
$$

Therefore, taking $V=A . d x$ and that the area $A$ is a constant over the integration domain, this yields:

$$
[\rho \phi]_{e}-[\rho \phi]_{w}=\left[\Gamma \frac{\partial \phi}{d x}\right]_{e}-\left[\Gamma \frac{\partial \phi}{d x}\right]_{w}+\int S d x
$$

in this study,

$$
\begin{gathered}
{\left[\Gamma \frac{\partial \phi}{\partial x}\right]_{e}=\Gamma_{e}\left(\frac{\phi_{E}-\phi_{P}}{\delta x_{P E}}\right)} \\
{\left[\Gamma \frac{\partial \phi}{\partial x}\right]_{w}=\Gamma_{w}\left(\frac{\phi_{P}-\phi_{W}}{\delta x_{W P}}\right)}
\end{gathered}
$$

The form of $[\phi]_{e}$ and $[\phi]_{w}$ are dependent on the finite-volume scheme chosen. Integration of the source term $S$ is trivial if, as in the case of this study, it is assumed to be constant across the control volume. This method is then applied to a computational grid, where linear methods are used to calculate $\phi$ at every location.

\section{A.2.1 The Hybrid Scheme}

The hybrid scheme [119] was devised to solve general stationary (time-independent) convection-diffusion problems, using the accuracy of second-order central differencing and the stability of the first-order upwind scheme. As a result, it has been incorporated into OSM to solve Ohm's law (A.10) and current conservation (A.11) equations, to calculate electrostatic potential profiles along field lines.

$$
\begin{gathered}
-\nabla_{\|} \phi=\eta j_{\|}-\frac{0.71}{e} \frac{d k T_{e}}{d s}-\frac{1}{e n} \frac{d p_{e}}{d s} \\
\nabla_{\|}\left(\frac{j_{\|}}{B}\right)=S_{j}
\end{gathered}
$$

where $\eta_{\|}$is the plasma electrical resistivity (ohm.m) $\left(\approx 2.7 \times 10^{-8} T_{e}(\mathrm{keV})^{-\frac{3}{2}}\right), j_{\|}$ is the electrical current density $\left(\mathrm{Am}^{-2}\right), \phi$ is the electrostatic potential $(\mathrm{V})$ and $p_{e}$ is the electron static pressure $(\mathrm{Pa})$. Combining these equations and eliminating $j_{\|}$yields a single convection-diffusion equation where $\phi$ is the only unknown: 


$$
\begin{aligned}
\nabla_{\|}^{2} \phi-\left(\frac{1}{\eta} \nabla_{\|} \eta+\frac{1}{B} \nabla_{\|} B\right) \nabla_{\|} \phi= & .71 \nabla_{\|}^{2} T_{e}-0.71 \nabla_{\|} T e\left(\frac{\nabla_{\|} B}{B}\right)-\frac{0.71}{\eta} \nabla_{\|} T e \nabla_{\|} \eta \\
& +\frac{1}{n_{e}} \nabla_{\|}^{2} p_{e}-\frac{1}{n_{e}^{2}} \nabla_{\|} p_{e} \nabla_{\|} n_{e}-\frac{1}{\eta n_{e}} \nabla_{\|} p_{e} \nabla_{\|} \eta \\
& -\frac{1}{n_{e}} \nabla_{\|} p_{e}\left(\frac{\nabla_{\|} B}{B}\right)-\eta S_{j}
\end{aligned}
$$

Furthermore, by using the relation:

$$
\vec{E}=-\vec{\nabla} \phi
$$

where $\vec{E}$ is the electrostatic field $\left(\mathrm{Vm}^{-1}\right)$ and all other terms have their usual meanings, the electrostatic field can be calculated from the resultant electrostatic potential profiles.

The versatility of this scheme can be demonstrated by taking a stationary convectiondiffusion equation:

$$
\frac{d}{d x}(\rho u \phi)=\frac{d}{d x}\left(\Gamma \frac{d \phi}{d x}\right)
$$

where $\rho$ is a mass density $\left(\mathrm{kg} / \mathrm{m}^{3}\right), u$ is a flow velocity $\left(\mathrm{ms}^{-1}\right), \Gamma$ is a diffusion coefficient $\left(\mathrm{m}^{2} \mathrm{~s}^{-1}\right)$ and $\phi$ is an unknown quantity. The properties of a system described by convection-diffusion equations, such as (A.12), can be described by the Peclet number, defined as:

$$
P e=\frac{\rho u \Delta x}{\Gamma}
$$

where $\Delta x$ is the grid spacing $(\mathrm{m})$. Therefore, the Peclet number is the ratio of the rate of convection and the rate of diffusion of the quantity $\phi$ in the system, and is related to the stability of some numerical schemes, as will be demonstrated. If a second-order central differencing scheme is applied to this problem, $[\rho \phi]_{e}$ and $[\rho \phi]_{w}$ terms in (A.27) are discretised as follows on a regularly-spaced grid:

$$
\begin{gathered}
{[\rho u \phi]_{e}=\rho_{e} u_{e}\left[\frac{\left(\phi_{P}+\phi_{E}\right)}{2}\right]} \\
{[\rho u \phi]_{w}=\rho_{w} u_{w}\left[\frac{\left(\phi_{W}+\phi_{P}\right)}{2}\right]}
\end{gathered}
$$

where the extrapolation of the unknown quantity to the cell faces is calculated by linear interpolation. In the following analysis, it is assumed that $\rho_{e}=\rho_{w}=\rho$ and 
$u_{e}=u_{w}=u$ for simplicity. To remove this constraint, the (known) mass density and velocity is linearly interpolated between cells in a similar way to $\phi$. The overall numerical scheme, in the absence of sources and sinks, can now be cast as:

$$
\rho u\left[\frac{\left(\phi_{P}+\phi_{E}\right)}{2}\right]-\rho u\left[\frac{\left(\phi_{W}+\phi_{P}\right)}{2}\right]=\Gamma\left(\frac{\phi_{E}-\phi_{P}}{\delta x}\right)-\Gamma\left(\frac{\phi_{P}-\phi_{W}}{\delta x}\right)
$$

The stability of this numerical scheme can be ascertained by varying $\rho, \Gamma$ and $u$ in order to change the Peclet number of the conservation equation.
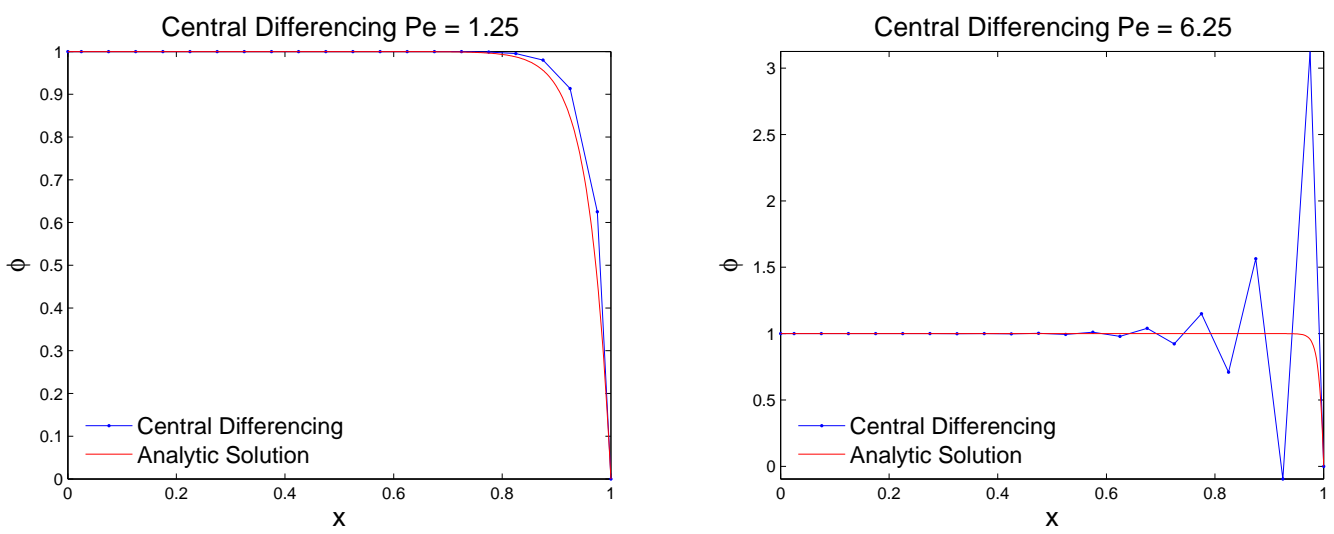

Figure A.4: Central differencing scheme used to solve a convection-diffusion problem (blue) compared to the analytic solution (red). The left figure shows a mildly diffusive case with Peclet number 1.25, in the right figure, the diffusive term has been weakened, increasing the Peclet number to 6.25, causing the solver to become unbounded.

When $P e=1.25$, the central differencing scheme produces a smooth profile in good agreement with the analytic solution to the problem (figure A.4). However, when $|P e| \geq 2$, the scheme becomes unstable and the solution produced unphysical. The reason for this behaviour is related to the boundedness of the scheme, which states that the value of $\phi$ should be bounded by it's boundary conditions. A necessary condition for a scheme to be bounded is that the coefficients of the terms in the discretised equations should have the same sign [118], normally positive. If the coefficient of the east cell calculation, $\phi_{E}$, is considered, for it to be positive for a positive flow velocity,

$$
\frac{\rho u \Delta x}{\Gamma}<2
$$

This can be re-cast as $\mathrm{Pe}<2$, explaining the apparent loss of stability of the scheme at high Peclet numbers. An alternative to the second-order central scheme is the firstorder upwind. In this scheme, the $[\rho \phi]_{e}$ and $[\rho \phi]_{w}$ terms in (A.27) are calculated using:

$$
[\rho u \phi]_{e}=\rho_{e} u_{e} \phi_{P}\left(u_{e}>0\right)
$$




$$
[\rho u \phi]_{w}=\rho_{w} u_{w} \phi_{W} \quad\left(u_{w}>0\right)
$$

alternatively,

$$
\begin{gathered}
{[\rho u \phi]_{e}=\rho_{e} u_{e} \phi_{E} \quad\left(u_{e}<0\right)} \\
{[\rho u \phi]_{w}=\rho_{w} u_{w} \phi_{P} \quad\left(u_{w}<0\right)}
\end{gathered}
$$

The main advantage of the first-order upwind scheme over the previous scheme is significantly enhanced stability, at the cost of reduced accuracy compared with the second-order central scheme, for low Peclet numbers. This is because the solution always satisfies the requirements for boundedness, resulting in the scheme being stable for all Peclet numbers. The first-order upwind scheme also smears out the presence of strong gradients in the solution (or "shocks"), thereby limiting its usefulness to cases where only smooth solutions are expected (figure A.5).
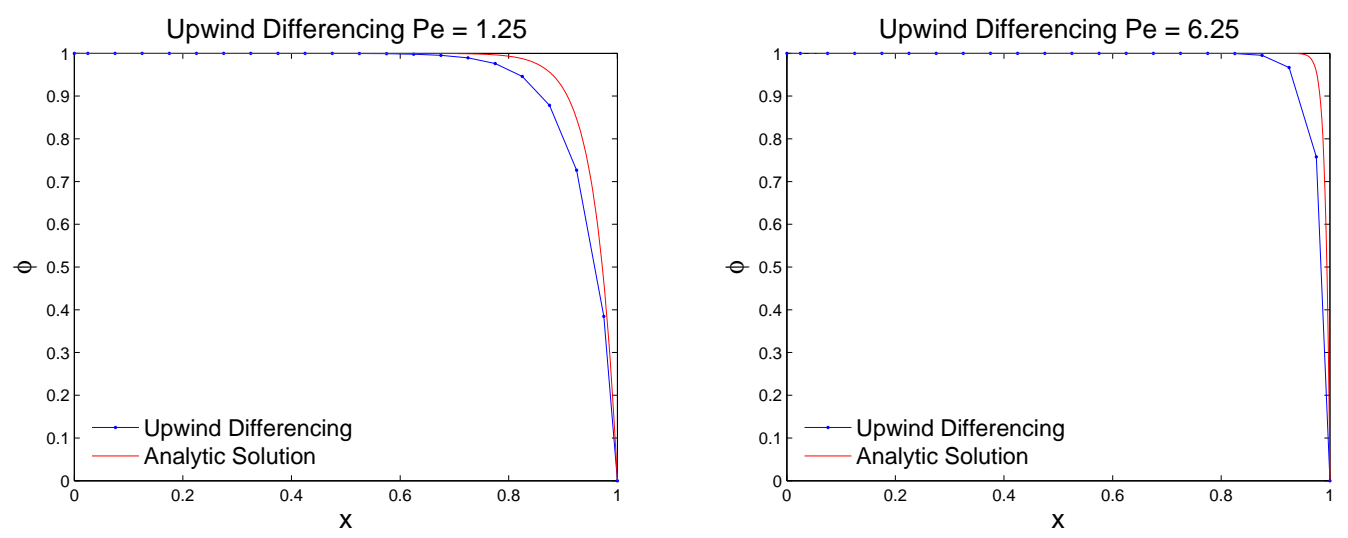

Figure A.5: The first order upwind differencing scheme applied to the same problem (blue) compared to the analytic solution (red). The scheme remains stable for all Peclet numbers, albeit at lower accuracy compared to the second-order central scheme for low Peclet numbers.

The hybrid scheme is a combination of the first-order upwind and second-order differencing schemes. For each cell, second-order central differencing is employed only if the scheme is stable $(\mathrm{Pe}<2)$, if not, the first-order upwind scheme is employed. The result is a scheme that is selectively more accurate where the Peclet number is low, and less accurate at higher Peclet numbers, but always stable. As stated above for the first-order upwind scheme, the ability of the hybrid scheme to resolve shocks that may be present in the solution is limited; and so it is best used in scenarios where the solution is highly unlikely to display such behaviour.

The application of the Hybrid scheme to the solution of Ohm's law and the current 

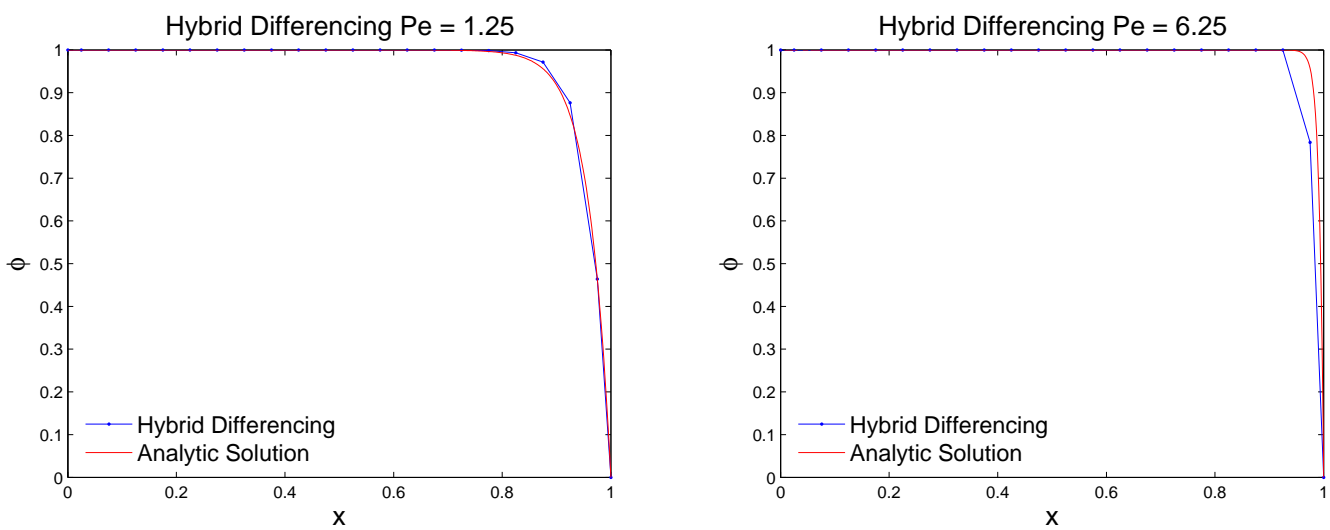

Figure A.6: The hybrid differencing scheme applied to the test problem (blue) compared to the analytic solution (red). Combining the accuracy of the central differencing and the stability of the upwind scheme can provide accurate results for problems with any Peclet number.

conservation equation in MAST shot 24861 at $240 \mathrm{~ms}$ produces electrostatic potential profiles in the scrape-off layer and private flux region. Discontinuities in $\phi$ exist close to material surfaces (at the edges of the simulation domain) as the plasma is accelerated by the pre-sheath electric field to satisfy the Bohm criterion. In this region, depending on the plasma conditions, the Hybrid scheme uses the $1^{\text {st }}$ order upwind discretisation in order to maintain stability. Elsewhere in the solution, the electrostatic potential is smoothly varying, and so the solver uses the more accurate $2^{\text {nd }}$ order central differencing scheme. Previous studies have shown that the Hybrid scheme produces reasonably accurate solutions when applied to 1D steady-state calculations only, and that significant artificial viscosity can occur if this scheme is used in higher dimensional time-varying contexts [120]. As a result, solution of the particle and momentum conservation equations are not attempted with this method.
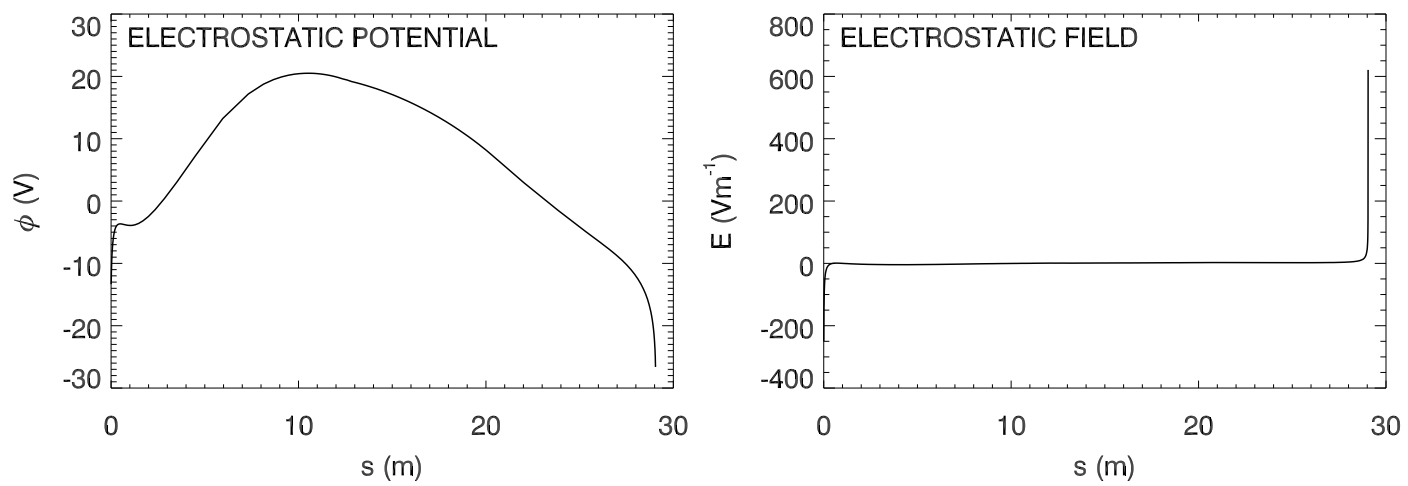

Figure A.7: Calculation of the electrostatic potential and electrostatic field for the plasma properties calculated in the steady-state solution shown in figure A.12. 


\section{A.2.2 Time-Dependent Equations}

Typically, finite-volume methods use linear operations in order to solve for a given quantity on a computational grid. Such methods are well-suited to solving systems of linear conservation laws, but less so if one or more is non-linear, which occurs frequently in fluid dynamics. Consequently, finite-volume (and other numerical schemes that rely on linear methods) rely on iterative schemes such as Newton-Raphson [121] when dealing with non-linear conservation laws.

As the OSM conservation equations are non-linear, an iterative scheme known as the pseudo-transient method [118] is used in this study. The method evolves a system of conservation laws in time until a steady-state is reached, which corresponds to the time-independent solution. Including time dependency in the original OSM equations yields:

$$
\begin{gathered}
\frac{\partial n_{e}}{\partial t}+\frac{\partial \Gamma_{e}}{\partial s}=S_{p} \\
m_{i} \frac{\partial \Gamma_{i}}{\partial t}+\frac{\partial\left(n_{e} T_{e}+n_{i} T_{i}+m_{i} \frac{\Gamma^{2}}{n_{i}}\right)}{\partial s}=S_{m}
\end{gathered}
$$

where $n_{e}=n_{i}\left(\Gamma_{i}=\Gamma_{e}\right)$ and $T_{e}=\gamma T_{i}$ as assumed previously (section 2.7). Including time-dependent behaviour in the finite-volume method requires a simple alteration of (A.27) to include an approximation of the time derivative operator. A common choice is:

$$
\frac{\partial \phi}{\partial t} \rightarrow \frac{\phi^{t+1}-\phi^{t}}{\Delta t}
$$

so a time-dependent finite-volume method can be cast as:

$$
\frac{\phi^{t+1}-\phi^{t}}{\Delta t}+[\rho \phi]_{e}^{t}-[\rho \phi]_{w}^{t}=\left[\Gamma \frac{\partial \phi}{d x}\right]_{e}^{t}-\left[\Gamma \frac{\partial \phi}{d x}\right]_{w}^{t}+\int S^{t} d x
$$

Convergence with this method is reached when balance is achieved between the convective and diffusive fluxes and sources. As a result, $\phi^{t+1}-\phi^{t} \rightarrow 0$ and the solution does not alter as time advances. Therefore, careful monitoring of how the solution behaves between consecutive time steps is important when monitoring whether the method has converged to a steady-state solution.

When solving time dependent systems of conservation equations, either implicit or explicit methods can be used. Explicit methods use information about a given quantity at the current time step to calculate the evolution of the solution to a subsequent time step. This method has the advantage of being more straightforward to implement, but care needs to be taken to ensure the numerical scheme remains stable, as will be 
elucidated later. Implicit methods mostly use information from the time step the solver is advancing towards, which is unknown, to advance the solution to a subsequent time step. The application of implicit numerical schemes is generally more complex, as a matrix inversion is required at each time step. However, implicit methods lack the constraint of stability criteria imposing a limit on the maximum permissible time step, which allow them to be more computationally efficient. However, implicit schemes are generally more difficult to implement for non-linear systems of conservation laws. The finite-volume solver used in this study is semi-implicit, meaning that the convective terms in the conservation equations are treated with an explicit scheme, and diffusive terms are treated with an implicit scheme.

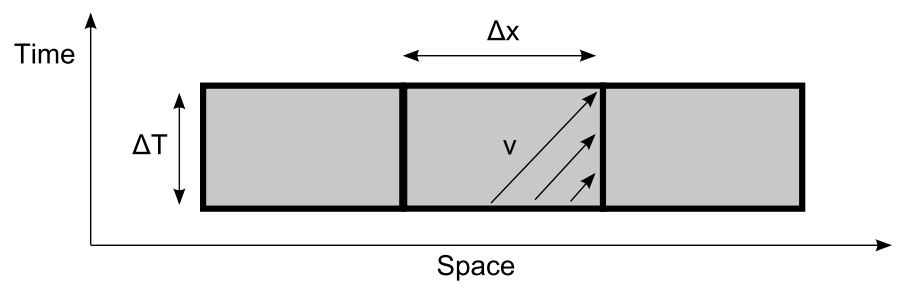

Figure A.8: The CFL condition limits the time step to prevent information from cells passing into adjacent ones

The principal limit on the admissible time step in the explicit calculations carried out in this study is the so-called Courant-Friedrichs-Lewy (CFL) condition [122]. In a system with no diffusive fluxes, information can only travel through the computational domain at a finite speed. If information propagates through a domain at speed $\mathrm{v}$, see figure A.8, then a time step larger than $\frac{\Delta x}{v}$ will result in information from one cell passing into a neighbouring cell. If a numerical scheme only considered information from one cell when making the calculation to advance the cell's contents through time, the information would be lost from the system if the CFL condition was not met. Therefore, in order to maintain the stability of an explicit numerical scheme, the following criterion is best obeyed:

$$
0 \leq \frac{v \Delta t}{\Delta x} \leq 1
$$

\section{A.2.3 The MUSCL Scheme}

MUSCL is a finite-volume discretisation of convective terms in partial differential equations. The full title of the scheme is the Monotone Upwind Schemes for Conservation Laws, formulated to provide accurate and stable solutions to systems of equations in the presence of steep gradient phenomena such as shocks [123]. In similar fashion to the hybrid scheme noted above, the MUSCL scheme utilises a second-order accurate discretisation where it is safe to do so, i.e. when the scheme is stable, but reduces 
the accuracy of the solution in regions where it is unstable. Unlike the hybrid scheme, however, the transition from second-order to first-order is not sudden, and is brought about by the application of special functions known as flux limiters. The purpose of the limiter is to impose a limit on the permissible gradient on the solution, as steep gradients resolved by second-order schemes are normally accompanied by spurious under- and overshoots as the scheme becomes unbounded. The flux limiters used here are not related to kinetic limits applied to fluid equations to prevent unphysical behaviour, such as that applied to electron heat conduction in the limit of low SOL collisionality [8].

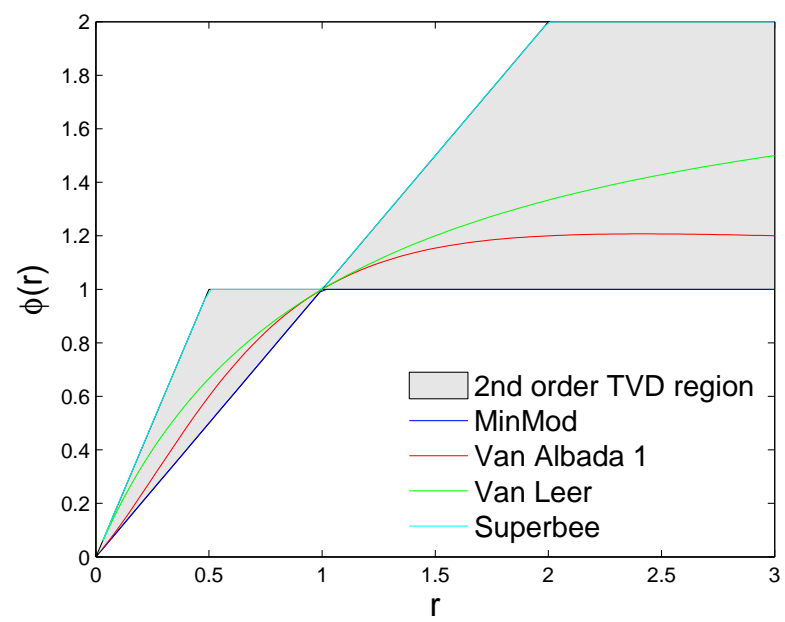

Figure A.9: Sweby diagram [124] indicating the effects of different flux limiters as a function of $r$, given by $r_{i}=\frac{\phi_{i}-\phi_{i-1}}{\phi_{i+1}-\phi_{i}}$. The MinMod limiter is the most aggressive at suppressing slopes in the solution, whereas the Superbee limiter is the least so.

The amplitude of some widely used flux limiters as a function of $r$, the backwarddifference gradient in the solution divided by the forward difference gradient, is shown in figure (A.9). The purpose of these limiters is to make the numerical scheme total variation diminishing, where the total variation is defined as:

$$
T V(\phi)=\sum_{i}\left|\phi_{i+1}-\phi_{i}\right|
$$

and a numerical scheme is total variation diminishing if:

$$
T V\left(\phi_{i+1}\right) \leq T V\left(\phi_{i}\right)
$$

In order to satisfy this condition, the scheme must not create any local extrema, and the value of a local minimum must be non-decreasing and that of a maximum must be non-increasing. The purpose of such schemes is to eliminate over- and undershoots that high-resolution numerical schemes create where the solution contains steep gradients 
(A.10).
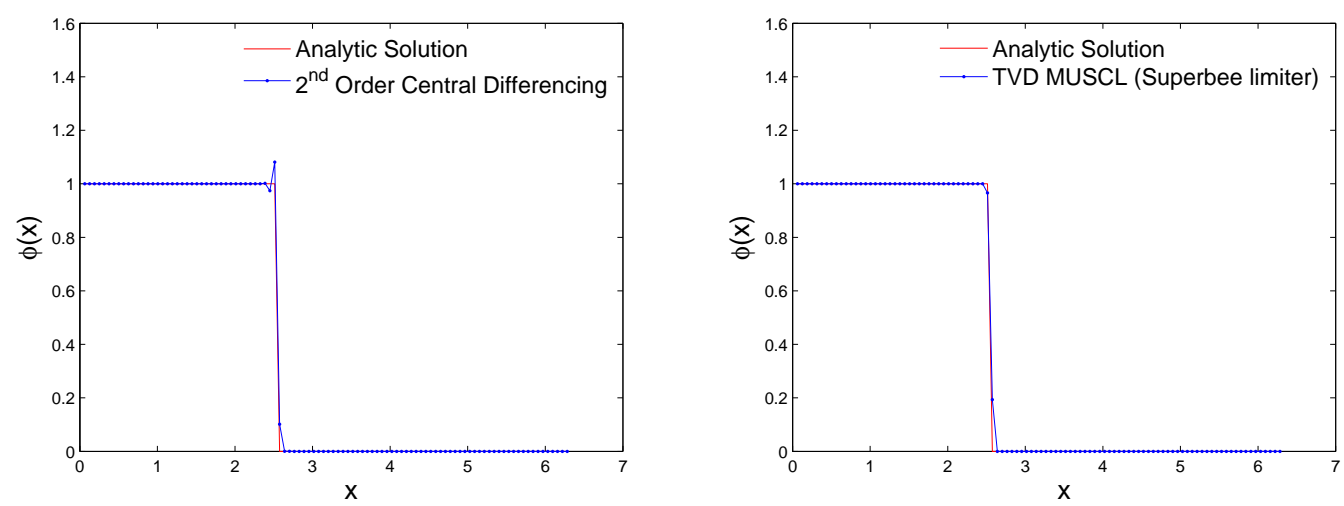

Figure A.10: In the presence of sharp discontinuities in the solution, some numerical schemes exhibit under- and overshoots (left). TVD schemes, however, are designed to resolve discontinuities without such behaviour.

In this study, the MUSCL scheme is employed to discretise the convective terms in time-dependent fluid particle and momentum conservation equations. This scheme is particularly apt when applied to the plasma boundary of Tokamaks, where the particle flux can experience a strong discontinuity close to material surfaces as the plasma accelerates to satisfy the Bohm criterion. The calculation of the convective flux in the MUSCL variant used in this study is based on the Kurganov-Tadmor (KT) scheme [125].

In applying the KT scheme, it is necessary to re-cast the conservation equations in the following form:

$$
\frac{\partial \phi}{\partial t}+\frac{\partial F}{\partial s}=S
$$

where $\phi$ is a vector of unknown quantities, $F$ is a vector of the convective flux of each unknown quantity, and $S$, is the source or sink of $\phi$. The convective term is then discretised to yield:

$$
\frac{\partial \phi}{\partial t}+\frac{1}{\Delta x_{i}}\left[F_{i+\frac{1}{2}}^{*}-F_{i-\frac{1}{2}}^{*}\right]=S
$$

where $F_{i+\frac{1}{2}}^{*}$ and $F_{i-\frac{1}{2}}^{*}$ are scheme-dependent fluxes of $\phi$ entering and leaving the cell across it's surfaces, respectively. In the KT scheme, these fluxes are given by:

$$
\begin{aligned}
& F_{i+\frac{1}{2}}^{*}=\frac{1}{2}\left[F\left(\phi_{i+\frac{1}{2}}^{+}\right)+F\left(\phi_{i+\frac{1}{2}}^{-}\right)-a_{i+\frac{1}{2}}\left(\phi_{i+\frac{1}{2}}^{+}-\phi_{i+\frac{1}{2}}^{-}\right)\right] \\
& F_{i-\frac{1}{2}}^{*}=\frac{1}{2}\left[F\left(\phi_{i-\frac{1}{2}}^{+}\right)+F\left(\phi_{i-\frac{1}{2}}^{-}\right)-a_{i-\frac{1}{2}}\left(\phi_{i-\frac{1}{2}}^{+}-\phi_{i-\frac{1}{2}}^{-}\right)\right]
\end{aligned}
$$

where $a_{i \pm \frac{1}{2}}$ is the maximum absolute value of the local information propagation 
speed, given by:

$$
a_{i \pm \frac{1}{2}}=\left|\max \left(\lambda_{i}, \lambda_{i \pm 1}\right)\right|
$$

where $\lambda_{i}$ is a vector of the eigenvalues of the Jacobian of the flux vector $\mathrm{F}$ and $\phi_{i+\frac{1}{2}}^{+}, \phi_{i+\frac{1}{2}}^{-}, \phi_{i-\frac{1}{2}}^{+}$and $\phi_{i-\frac{1}{2}}^{-}$are given, on an evenly-spaced grid, by:

$$
\begin{aligned}
\phi_{i+\frac{1}{2}}^{+} & =\phi_{i+1}-\frac{\phi\left(r_{i+1}\right)}{2}\left(\phi_{i+2}-\phi_{i+1}\right) \\
\phi_{i+\frac{1}{2}}^{-} & =\phi_{i}+\frac{\phi\left(r_{i}\right)}{2}\left(\phi_{i+1}+\phi_{i}\right) \\
\phi_{i-\frac{1}{2}}^{+} & =\phi_{i}-\frac{\phi\left(r_{i}\right)}{2}\left(\phi_{i+1}-\phi_{i}\right) \\
\phi_{i-\frac{1}{2}}^{-} & =\phi_{i-1}+\frac{\phi\left(r_{i-1}\right)}{2}\left(\phi_{i}-\phi_{i-1}\right)
\end{aligned}
$$

where $\phi(r)$ is a limiter function and $\phi_{i}$ is an unknown quantity to be calculated at the centre of cell $i$. To test the accuracy and suitability of this numerical scheme for handling non-linear conservation equations, the solution of inviscid Burgers' equation (A.37) was calculated with a sinusoidal wave as an initial condition, as in [125].

$$
\frac{\partial u}{\partial t}+u \frac{\partial u}{\partial x}=0
$$

This common test problem is known to develop a shock at $\mathrm{T}=1 \mathrm{~s}$, as a result, a comparison between the analytical and computed solutions was made at $\mathrm{T}=2 \mathrm{~s}$ (figure A.11). The agreement between the two solutions is good, even around the shock, where the sharpness of the discontinuity has been smoothed partly by the application of the flux limiter, but also because of the grid resolution used in this simulation. Running the code with finer grid resolution steepens the discontinuity close to the shock, although also requiring smaller time steps in order to maintain stability.

\section{A.3 The Time-Dependent Original Model}

The time-dependent version of the original OSM model is given by:

$$
\begin{gathered}
\frac{\partial n_{e}}{\partial t}+\frac{\partial \Gamma_{e}}{\partial s}=S_{p} \\
m_{i} \frac{\partial \Gamma_{i}}{\partial t}+\frac{\partial\left(n_{e} T_{e}+n_{i} T_{i}+m_{i} \frac{\Gamma_{i}^{2}}{n_{i}}\right)}{\partial s}=S_{m}
\end{gathered}
$$

where, as before, $n_{e}=n_{i}$ and $\Gamma_{e}=\Gamma_{i}$. Using the MUSCL scheme to discretise the 


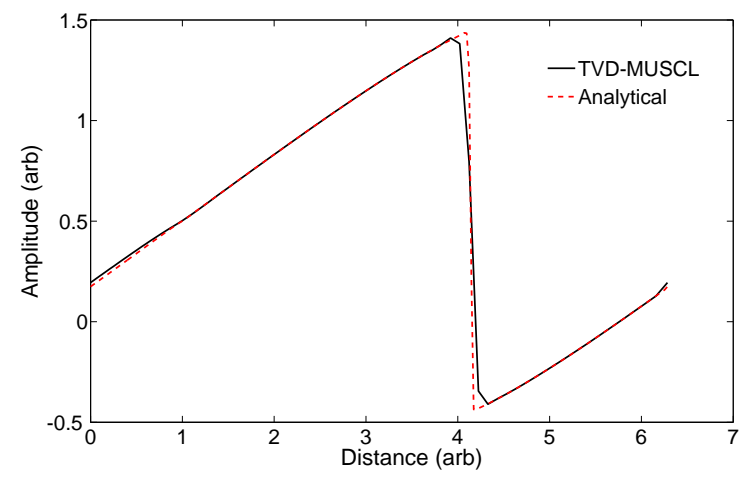

Figure A.11: Comparison between the analytical and computed solutions of the inviscid Burgers' equation after the formation of a shock.

convective term and a Runge-Kutta method to carry out time-stepping, it is possible to calculate (A.38) and (A.39) the stationary solution of the equations via the pseudotransient method. In order to formulate the above equations to be compatible with the MUSCL scheme, the above model is re-cast into the form of a convection equation (A.31) as follows:

$$
\begin{aligned}
\phi_{i} & =\left[\begin{array}{c}
n_{i} \\
m_{i} \Gamma_{i}
\end{array}\right] \\
F_{i} & =\left[\begin{array}{c}
\Gamma_{i} \\
n_{i}\left(T_{e}+T_{i}\right)+\frac{m_{i} \Gamma_{i}^{2}}{n_{i}}
\end{array}\right] \\
S_{i} & =\left[\begin{array}{l}
S_{p} \\
S_{m}
\end{array}\right]
\end{aligned}
$$

and the eigenvalues of $F_{i}$ are given by:

$$
\lambda_{i}=\left[\begin{array}{l}
\frac{\Gamma_{i}}{n_{i}}+\sqrt{\frac{T_{e}+T_{i}}{m_{i}}} \\
\frac{\Gamma_{i}}{n_{i}}-\sqrt{\frac{T_{e}+T_{i}}{m_{i}}}
\end{array}\right]
$$

where all terms above have their usual meanings. Initial "guessed" profiles of $n_{e}$ and $\Gamma_{e}$ are used to commence the time-stepping procedure, and the quantities of interest are evolved in time until they relax to a state where the time evolution no longer alters the system. At this point, it can be said that the solver has converged upon the stationary solution of the equations.

To carry out the aforementioned time-stepping, a low-storage Runge-Kutta scheme known as the SHK (Sommeijer, van der Houwen and Kok) method is used [126], which is given by: 


$$
\begin{aligned}
\phi_{i}^{*} & =\phi_{i}^{n}-\alpha_{1} \tau L_{h} \phi_{i}^{n} \\
\phi_{i}^{* *} & =\phi_{i}^{n}-\alpha_{2} \tau L_{h} \phi_{i}^{*} \\
\phi_{i}^{* * *} & =\phi_{i}^{n}-\frac{1}{2} \tau L_{h} \phi_{i}^{* *} \\
\phi_{i}^{n+1} & =\phi_{i}^{n}-\tau L_{h} \phi_{i}^{* * *}
\end{aligned}
$$

where $\alpha_{1}=\frac{1}{4}$ and $\alpha_{2}=\frac{1}{3}, \tau$ is the time interval over which the solution $\rho$ is being stepped and $L_{h} \rho^{n}$ is the spatial discretisation of $\rho$. The SHK method was designed for convection-diffusion problems, and offers good stability in convection dominated flows.

Imposition of boundary conditions is treated by exploiting the assumption that the parallel ion velocity at the divertor plates is at least the local sound speed [8] when $T_{i} \neq T_{e}$. In this numerical scheme, it is assumed that the parallel ion velocity is equal to the sound speed at the cell surface intersecting a material surface at the end of a flux tube. As two first-order partial differential equations are considered, for a flux tube terminated by material surfaces at both ends, this condition is sufficient to calculate a unique solution to the conservation equations (A.38) \& (A.39).
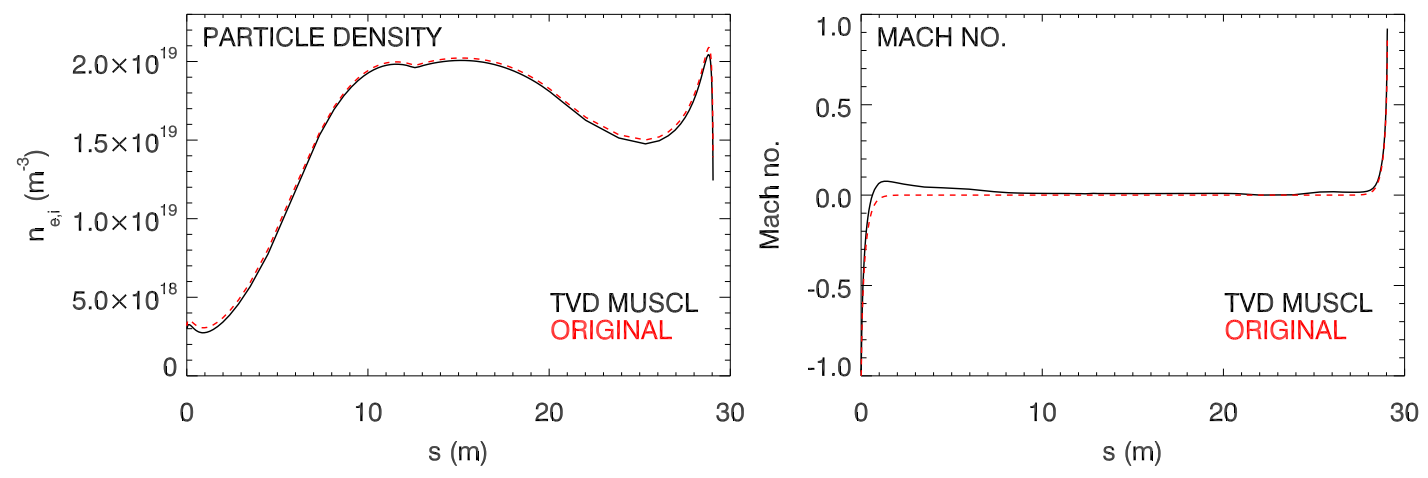

Figure A.12: Comparison of steady-state electron density (left) and ion Mach number (right) in the primary separatrix of MAST shot 24861 at $240 \mathrm{~ms}$.

Comparison of the solutions calculated using the original and MUSCL solvers reveals a similarity between the ion velocities and a systematic decrease in electron density with the MUSCL solver. The cause of the dissimilarity is due to the nature of the limiter, reducing the gradients found in the solution to maintain stability. A careful choice of limiter can reduce the difference between the answers yielded by the two methods to some degree, but the presence of a limiter result in differences between the calculated solutions. In the MUSCL scheme, the solution evolves from a trial solution to a steady-state in a few milliseconds $(\approx 3-4 \mathrm{~ms})$, which is consistent with the notion that information is propagated through the solution at the local sound speed, $\sim 10^{4} \mathrm{~ms}^{-1}$. 

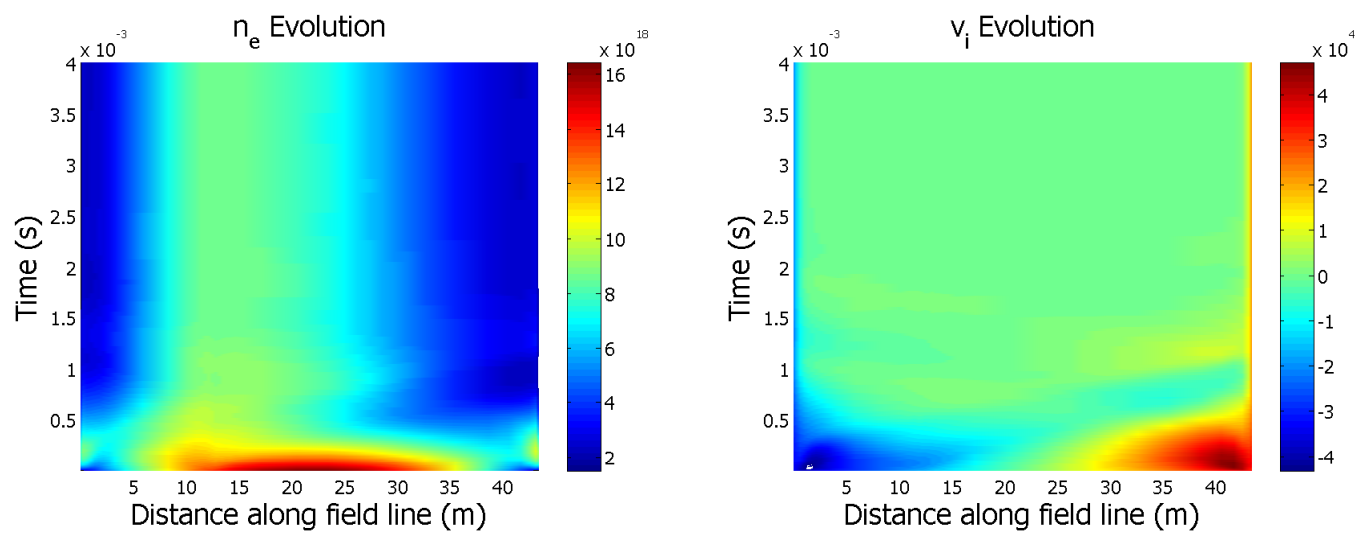

Figure A.13: Density and ion velocity evolution during a TVD-MUSCL solver run Evolution of electron density (left) and ion velocity (right) profiles with time. The solution relaxes from a guessed profile to a steady-state solution after $\sim 3 \mathrm{~ms}$.
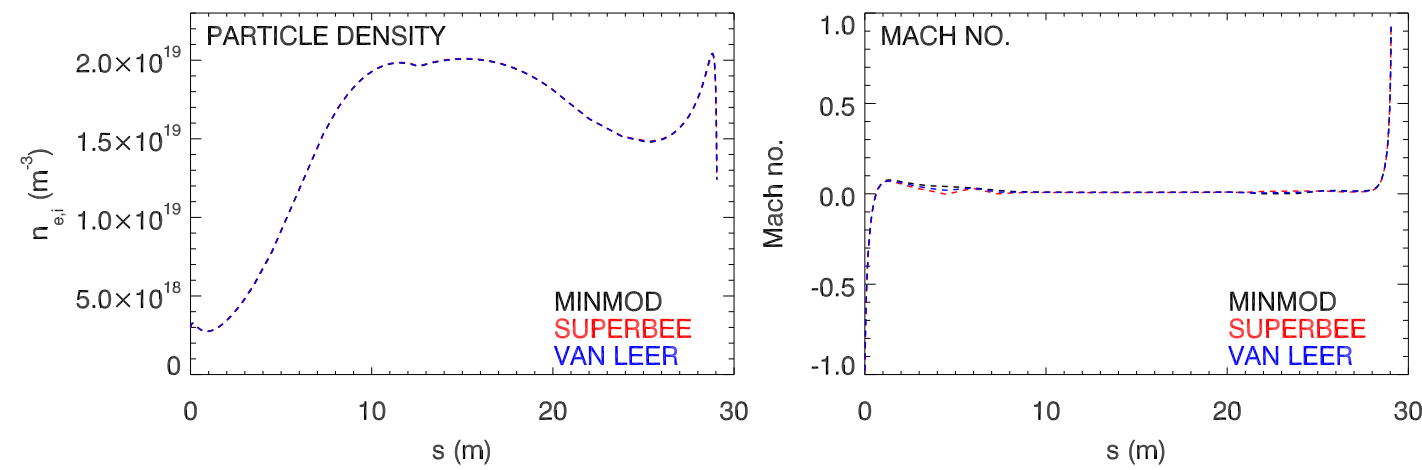

Figure A.14: Comparison of the steady-state electron density (left) and ion Mach number (right) computed with the TVD MUSCL solver using different flux limiters.

The effects of different flux limiters on the steady-state plasma solution was found by running OSM with the same initial conditions, but with different flux limiters enabled. In order to get a sense for how the solution varies, limiters were chosen at the two extremes of the Sweby diagram, namely the MinMod and Superbee limiters, and with the van Leer limiter, that lies approximately mid-way between the two extremes in the $2^{\text {nd }}$ order TVD region (figure A.9). The solutions yielded (figure A.14) show that the calculated density using the linear particle conservation equation are very similar with all of the limiters, but the ion Mach number diverges at the inner divertor leg (s $\approx 4 \mathrm{~m}$ ) briefly, before converging beyond $\mathrm{s} \approx 9 \mathrm{~m}$. An explanation for the divergence between the plasma solutions occurring at this location is that the numerical scheme is asymmetrical in space, and so the numerical solver behaves differently approaching steep gradients (at the high $\mathrm{s}$ target) compared with relaxing from them (at the low $\mathrm{s}$ target). 


\section{A.4 The Extended Time-Dependent Model}

The handling of diffusive transport terms in a finite-volume is more straightforward in terms of spatial discretisation, as such terms do not suffer from instabilities that are common for convective terms. However, it isn't practical to use an explicit formulation of diffusive transport, as the stability criterion scales roughly as $1 /(\Delta x)^{2}$, compared with $1 / \Delta x$ for convective transport. Therefore, on finely-spaced grids where $\Delta x$ is small, the number of time steps required to reach convergence rises significantly. Therefore, it is necessary to use an implicit formulation for such terms. The incorporation of diffusive transport into the original OSM model (A.43) allows the effects of parallel viscosity to be explored, the effects of which are elucidated in section 4.4.

$$
m_{i} \frac{\partial \Gamma_{i}}{\partial t}+\frac{\partial}{\partial s}\left(n_{e} T_{e}+n_{i} T_{i}+m_{i} \frac{\Gamma_{i}^{2}}{n_{i}}\right)-\frac{\partial}{\partial s}\left(\eta_{\|} \frac{\partial}{\partial s}\left(\frac{\Gamma_{i}}{n_{i}}\right)\right)=S_{m}
$$

where $\eta$ is the parallel diffusion coefficient, given the diffusive term can be expanded to give:

$$
\frac{\partial}{\partial s}\left(\eta_{\|} \frac{\partial}{\partial s}\left(\frac{\Gamma_{i}}{n_{i}}\right)\right)=\frac{\partial}{\partial s}\left(\frac{\eta_{\|}}{n_{e}} \frac{\partial \Gamma_{i}}{\partial s}-\frac{\eta_{\|} \Gamma_{i}}{n_{i}^{2}} \frac{\partial n_{i}}{\partial s}\right)
$$

where the coefficients in font of $\frac{\partial \Gamma_{i}}{\partial s}$ and $\frac{\partial n_{i}}{\partial s}$ are both calculated at the current time step, and the partial derivative of the ion flux $\Gamma_{i}$ and ion density $n_{i}$ are calculated implicitly. As a result, this calculation somewhat reduces the accuracy of time-dependent calculations, but does not affect the steady state solution reached when the numerical solver converges. Implementing diffusive terms in OSM in this way has been found to have no effect on the solver solutions in the case of zero diffusion coefficient, and agree well with an explicit implementation (not shown). It has also been found that this implementation has no adverse effects on the time step required to ensure solver stability. 


\section{References}

[1] S. W. Lisgo, Interpretive Modeling of the Alcator C-Mod Divertor. PhD thesis, University of Toronto, 2003.

[2] D. Reiter. The EIRENE code, www.eirene.de.

[3] J. Wesson, Tokamaks, ch. 1, p. 5. Clarendon press, 3 ed., 2004.

[4] Y.-K. M. Peng and D. J. Strickler Nuclear Fusion, vol. 26, p. 769, 1986.

[5] J. Friedberg, Plasma Physics and Fusion Energy. Cambridge, 2007.

[6] A. Sykes, the START Team, the NBI Team, the MAST Team, and the Theory Team, "The spherical tokamak programme at Culham," Nuclear Fusion, vol. 39, no. 9Y, p. 1271, 1999.

[7] G. F. Matthews, "Plasma detachment from divertor targets and limiters," Journal of Nuclear Materials, vol. 220-222, pp. 104 - 116, 1995. Plasma-Surface Interactions in Controlled Fusion Devices.

[8] P. C. Stangeby, The Plasma Boundary of Magnetic Fusion Devices. IoP Publishing, 2000.

[9] ITER Physics Expert Group on Divertor and ITER Physics Expert Group on Divertor Modelling and Database and ITER Physics Basis Editors, "Chapter 4: Power and particle control," Nuclear Fusion, vol. 39, no. 12, pp. 2391-2469, 1999.

[10] A. Loarte, "Effects of divertor geometry on tokamak plasmas," Plasma Physics and Controlled Fusion, vol. 43, no. 6, pp. R183-R224, 2001.

[11] C. S. Pitcher and P. C. Stangeby, "Experimental divertor physics," Plasma Physics and Controlled Fusion, vol. 39, no. 6, pp. 779-930, 1997.

[12] R. Schneider, H. S. Bosch, D. Coster, J. C. Fuchs, J. Gafert, G. Haas, A. Herrmann, M. Kaufmann, A. Kallenbach, J. Neuhauser, J. Schweinzer, U. Wenzel, and A. U. Teams, "Role of divertor geometry on detachment in 
ASDEX Upgrade," Journal of Nuclear Materials, vol. 266-269, pp. 175 - 181, 1999.

[13] D. P. Coster, K. Borrass, and R. Schneider, "B2-eirene modelling of the density limit on ASDEX-Upgrade," Journal of Nuclear Materials, vol. 266-269, pp. 804 $-808,1999$.

[14] JET Team and R. Monk, "Recent results from divertor and scrape-off layer studies at JET," Nuclear Fusion, vol. 39, no. 11Y, p. 1751, 1999.

[15] P. C. Stangeby, "A tutorial on some basic aspects of divertor physics," Plasma Physics and Controlled Fusion, vol. 42, no. 12B, pp. B271-B291, 2000.

[16] D. Bohm, The Characteristics of Electrical Discharges in Magnetic Fields. McGraw-Hill, 1949.

[17] B. Lipschultz, J. L. Terry, C. Boswell, A. Hubbard, B. LaBombard, and D. A. Pappas, "Ultrahigh densities and volume recombination inside the separatrix of the Alcator C-Mod tokamak," Phys. Rev. Lett., vol. 81, pp. 1007-1010, Aug 1998.

[18] A. Loarte, R. Monk, J. Martin-Solis, D. Campbell, A. Chankin, S. Clement, S. Davies, J. Ehrenberg, S. Erents, H. Guo, P. Harbour, L. Horton, L. Ingesson, H. Jackel, J. Lingertat, C. Lowry, C. Maggi, G. Matthews, K. McCormick, D. O'Brien, R. Reichle, G. Saibene, R. Smith, M. Stamp, D. Stork, and G. Vlases, "Plasma detachment in JET mark I divertor experiments," Nuclear Fusion, vol. 38, no. 3, pp. 331-371, 1998.

[19] P. Stangeby, "Can detached divertor plasmas be explained as self-sustained gas targets?," Nuclear Fusion, vol. 33, no. 11, pp. 1695-1705, 1993.

[20] D. Lumma, J. L. Terry, and B. Lipschultz, "Radiative and three-body recombination in the Alcator C-Mod divertor," Physics of Plasmas, vol. 4, no. 7, pp. 25552566, 1997.

[21] M. E. Fenstermacher, "Physics of the detached radiative divertor regime in DIIID," Plasma Physics and Controlled Fusion, vol. 41, no. 3A, pp. A345-A355, 1999.

[22] R. Pitts, B. Duval, A. Loarte, J.-M. Moret, J. Boedo, D. Coster, I. Furno, J. Horacek, A. Kukushkin, D. Reiter, and J. Rommers, "Divertor geometry effects on detachment in TCV," Journal of Nuclear Materials, vol. 290-293, pp. 940 946, 2001. 14th Int. Conf. on Plasma-Surface Interactions in Controlled Fusion D evices. 
[23] D. R. Baker, R. T. Snider, and M. Nagami, "Observation of cold high-density plasma near the Doublet III limiter," Nuclear Fusion, vol. 22, p. 807, 1982.

[24] W. Fundamenski, "Power and particle exhaust in tokamaks: Integration of plasma scenarios with plasma facing materials and components," Journal of Nuclear Materials, vol. 390-391, pp. 10 - 19, 2009. Proceedings of the 18th International Conference on Plasma-Surface Interactions in Controlled Fusion Device, Proceedings of the 18th International Conference on Plasma-Surface Interactions in Controlled Fusion Device.

[25] I. Hutchinson, "Thermal front analysis of detached divertors and MARFEs," Nuclear Fusion, vol. 34, no. 10, pp. 1337-48, 1994.

[26] G. F. Counsell, "The plasma-wall interaction region: a key low temperature plasma for controlled fusion," Plasma Sources Science and Technology, vol. 11, no. 3A, pp. A80-A85, 2002.

[27] L. Horton, G. Vlases, P. Andrew, V. Bhatnagar, A. Chankin, S. Clement, G. Conway, S. Davies, J. de Haas, J. Ehrenberg, G. Fishpool, E. Gauthier, H. Guo, P. Harbour, L. Ingesson, H. Jackel, J. Lingertat, A. Loarte, C. Lowry, C. Maggi, G. Matthews, G. McCracken, R. Mohanti, R. Monk, R. Reichle, E. Righi, G. Saibene, R. Sartori, R. Simonini, M. Stamp, A. Taroni, and K. Thomsen, "Studies in JET divertors of varied geometry. I: Non-seeded plasma operation," Nuclear Fusion, vol. 39, no. 1, pp. 1-17, 1999.

[28] V. Rozhansky, S. Voskoboynikov, E. Kaveeva, D. Coster, and R. Schneider, "Simulation of tokamak edge plasma including self-consistent electric fields," Nuclear Fusion, vol. 41, no. 4, p. 387, 2001.

[29] G. J. Radford, A. V. Chankin, G. Corrigan, R. Simonini, J. Spence, and A. Taroni, "The particle and heat drift fluxes and their implementation into the EDGE2D transport code," Contributions to Plasma Physics, vol. 36, pp. 187-191, 1996.

[30] T. D. Rognlien, D. D. Ryutov, N. Mattor, and G. D. Porter, "Two-dimensional electric fields and drifts near the magnetic separatrix in divertor tokamaks," Physics of Plasmas, vol. 6, no. 5, pp. 1851-1857, 1999.

[31] X. Q. Xu and R. H. Cohen, "Scrape-off layer turbulence theory and simulations," Contributions to Plasma Physics, vol. 38, pp. 158-170, 1998.

[32] P. Tamain, P. Ghendrih, E. Tsitrone, V. Grandgirard, X. Garbet, Y. Sarazin, E. Serre, G. Ciraolo, and G. Chiavassa, "TOKAM-3D: A 3D fluid code 
for transport and turbulence in the edge plasma of tokamaks," Journal of Computational Physics, vol. 229, no. 2, pp. 361 - 378, 2010.

[33] S. W. Lisgo, G. F. Counsell, P. Boerner, J. Dowling, A. Kirk, G. Maddison, M. Price, D. Reiter, R. Scannell, and the MAST Team, "Calculation of the MAST pedestal ionisation profile from boundary plasma reconstruction," in Proceedings of the 34th EPS conference on plasma physics, 2007.

[34] W. Fundamenski, S. Erents, G. Matthews, A. Chankin, V. Riccardo, P. Stangeby, and J. Elder, "Analysis of SOL behaviour in JET MkIIGB using an advanced onion-skin solver (OSM2)," Journal of Nuclear Materials, vol. 290-293, pp. 593 - 597, 2001. 14th Int. Conf. on Plasma-Surface Interactions in Controlled Fusion Devices.

[35] M. Cox, "The Mega Amp Spherical Tokamak," Fusion Engineering and Design, vol. 46, no. 2-4, pp. $397-404,1999$.

[36] J. Friedberg, Plasma Physics and Fusion Energy, ch. 11, p. 271. Cambridge, 2007.

[37] A. Darke, R. Hayward, G. Counsell, and K. Hawkins, "The MAST improved divertor," Fusion Engineering and Design, vol. 75-79, pp. 285 - 289, 2005. Proceedings of the 23rd Symposium of Fusion Technology - SOFT 23.

[38] A. Kirk, G. F. Counsell, W. Fundamenski, J.-W. Ahn, D. Taylor, M. J. Walsh, Y. Yang, and the MAST team, "A comparison of mid-plane scrape-off-layer measurements with model predictions in MAST and the calculation of cross-field transport coefficients," Plasma Physics and Controlled Fusion, vol. 46, no. 10, p. 1591, 2004.

[39] G. Counsell, J.-W. Ahn, R. Cohen, A. Kirk, P. Helander, R. Martin, D. Ryutov, A. Tabasso, H. Wilson, Y. Yang, and the MAST team, "Exhaust, ELM, and halo physics using the MAST tokamak," Nuclear Fusion, vol. 43, no. 10, p. 1197, 2003.

[40] G. D. Temmerman, E. Delchambre, J. Dowling, A. Kirk, S. Lisgo, and P. Tamain, "Thermographic study of heat load asymmetries during MAST L-mode discharges," Plasma Physics and Controlled Fusion, vol. 52, no. 9, p. 095005, 2010.

[41] M. A. Lieberman and A. J. Lichtenberg, Principles of plasma discharges and materials processing, ch. 6, p. 175. Wiley, 1994.

[42] P. Tamain, A. Kirk, E. Nardon, B. Dudson, B. Hnat, and the MAST team, "Edge turbulence and flows in the presence of resonant magnetic perturbations 
on MAST," Plasma Physics and Controlled Fusion, vol. 52, no. 7, p. 075017, 2010 .

[43] J. Ahn, Investigations of the Boundary Plasma in the MAST Spherical Tokamak. $\mathrm{PhD}$ thesis, Imperial College of Science, Technology and Medicine, 2002.

[44] I. H. Hutchinson, Principles of Plasma Diagnostics, ch. 6, p. 261. Cambridge, 2002.

[45] C. Stehlé and R. Hutcheon, "Extensive tabulations of Stark broadened hydrogen line profiles," Astron. Astrophys. Suppl. Ser., vol. 140, pp. 93-97, nov 1999.

[46] U. Fantz, "Basics of plasma spectroscopy," Plasma Sources Science and Technology, vol. 15, no. 4, p. S137, 2006.

[47] J. M. Hollas, Modern Spectroscopy, ch. 2, pp. 28-30. Wiley, 2004.

[48] I. H. Hutchinson, Principles of Plasma Diagnostics. Cambridge, 2 ed., 2002.

[49] H. R. Griem, Principles of plasma spectroscopy, ch. 7, p. 193. Cambridge, 2005.

[50] I. H. Hutchinson, Principles of Plasma Diagnostics, ch. Appendix 2, p. 405. Cambridge, 2002.

[51] H. R. Griem, Principles of Plasma Spectroscopy. Cambridge University Press, 2005.

[52] H. R. Griem, Spectral line broadening by plasmas. Academic press, 1974.

[53] N. Konjevic and J. R. Roberts, "A critical review of the stark widths and shifts of spectral lines from non-hydrogenic atoms," Journal of Physical and Chemical Reference Data, vol. 5, pp. 209-527, 1976.

[54] P. Barklem, N. Piskunov, and B. O'Mara, "Self-broadening in balmer line wing formation in stellar atmospheres," Astron. Astrophys., vol. 363, pp. 1091-1105, 2000 .

[55] G. Drake, Springer handbook of atomic, molecular, and optical physics. Springer, 2 ed., 2006.

[56] R. Scannell, M. J. Walsh, P. G. Carolan, A. C. Darke, M. R. Dunstan, R. B. Huxford, G. McArdle, D. Morgan, G. Naylor, T. O'Gorman, S. Shibaev, N. Barratt, K. J. Gibson, G. J. Tallents, and H. R. Wilson, "Design of a new Nd:YAG Thomson scattering system for MAST," vol. 79, p. 10E730, AIP, 2008. 
[57] R. Scannell, A. Kirk, N. B. Ayed, P. G. Carolan, G. Cunningham, J. McCone, S. L. Prunty, and M. J. Walsh, "Experimental investigation into ELM filament formation on MAST," Plasma Physics and Controlled Fusion, vol. 49, no. 9, p. 1431, 2007.

[58] A. Kirk, G. F. Counsell, G. Cunningham, J. Dowling, M. Dunstan, H. Meyer, M. Price, S. Saarelma, R. Scannell, M. Walsh, H. R. Wilson, and the MAST team, "Evolution of the pedestal on MAST and the implications for ELM power loadings," Plasma Physics and Controlled Fusion, vol. 49, no. 8, p. 1259, 2007.

[59] J. K. Anderson, P. L. Andrew, B. E. Chapman, D. Craig, and D. J. D. Hartog, "Direct removal of edge-localized pollutant emission in a near-infrared bremsstrahlung measurement," Review of Scientific Instruments, vol. 74, no. 3, pp. 2107-2110, 2003.

[60] S. Lisgo, P. Brner, G. Counsell, J. Dowling, A. Kirk, R. Scannell, M. O'Mullane, and D. Reiter, "Interpretation of spatially resolved helium line ratios on MAST," Journal of Nuclear Materials, vol. 390-391, pp. 1078 - 1080, 2009. Proceedings of the 18th International Conference on Plasma-Surface Interactions in Controlled Fusion Device, Proceedings of the 18th International Conference on PlasmaSurface Interactions in Controlled Fusion Device.

[61] R. C. Aster, B. Borchers, and C. Thurber, Parameter Estimation and Inverse Problems. Elsevier, 2005.

[62] L. L. Lao, H. S. John, R. D. Stambaugh, A. G. Kellman, and W. Pfeiffer Nuclear Fusion, vol. 25, p. 1611, 1985.

[63] R. Marchand and M. Dumberry, "CARRE: a quasi-orthogonal mesh generator for 2D edge plasma modelling," Computer Physics Communications, vol. 96, no. 2-3, pp. $232-246,1996$.

[64] V. Rozhansky, E. Kaveeva, S. Voskoboynikov, D. Coster, X. Bonnin, and R. Schneider, "Potentials and currents in the edge tokamak plasma: simplified approach and comparison with two-dimensional modelling," Nuclear Fusion, vol. 43, no. 7 , p. $614,2003$.

[65] P. J. Harbour, "Current flow parallel to the field in a scrape-off layer," Contributions to Plasma Physics, vol. 28, no. 4-5, pp. 417-419, 1988.

[66] J. Harrison, S. Lisgo, G. Counsell, K. Gibson, J. Dowling, L. Trojan, and D. Reiter, "Interpretive modelling of scrape-off plasmas on the MAST tokamak," Journal of Nuclear Materials, vol. 390-391, pp. 392 - 394, 2009. Proceedings of 
the 18th International Conference on Plasma-Surface Interactions in Controlled Fusion Device, Proceedings of the 18th International Conference on PlasmaSurface Interactions in Controlled Fusion Device.

[67] W. H. Press, S. A. Teukolsky, W. T. Vetterling, and B. P. Flannery, Numerical Recipes in Fortran 77890 - The Art of Scientific Computing, vol. 1. Cambridge, 2 ed., 1992.

[68] A. Kumagai, N. Asakura, K. Itami, M. Shimada, and M. Nagami, "Parallel currents in the scrape-off layer of high-density JT-60U discharges," Plasma Physics and Controlled Fusion, vol. 39, no. 8, p. 1189, 1997.

[69] A. Kallenbach, A. Carlson, G. Pautasso, A. Peeters, U. Seidel, and H.-P. Zehrfeld, "Electric currents in the scrape-off layer in ASDEX Upgrade," Journal of Nuclear Materials, vol. 290-293, pp. 639 - 643, 2001. 14th Int. Conf. on Plasma-Surface Interactions in Controlled Fusion D evices.

[70] C. G. Silva, S. J. Fielding, K. B. Axon, and M. G. Booth, "SOL currents and divertor asymmetries on COMPASS-D," Journal of Nuclear Materials, vol. 266269, pp. $679-684,1999$.

[71] A. Chankin, S. Clement, L. de Kock, S. Erents, P. Harbour, and J. Tagle, "Parallel currents in the scrape-off layer of JET diverted discharges," Journal of Nuclear Materials, vol. 196-198, pp. 739 - 744, 1992. Plasma-Surface Interactions in Controlled Fusion Devices, Proceedings of the Tenth International Conference on Plasma-Surface Interactions in Controlled Fusion Devices.

[72] A. V. Chankin and P. C. Stangeby, "Particle and parallel momentum balance equations with inclusion of drifts, for modelling strong- to weakly-collisional edge plasmas," Nuclear Fusion, vol. 46, no. 12, p. 975, 2006.

[73] A. Kirk, W. Fundamenski, J.-W. Ahn, and G. Counsell, "Parallel SOL transport in MAST and JET: the impact of the mirror force," Plasma Physics and Controlled Fusion, vol. 45, no. 8, p. 1445, 2003.

[74] N. Asakura, "Understanding the SOL flow in L-mode plasma on divertor tokamaks, and its influence on the plasma transport," Journal of Nuclear Materials, vol. 363-365, pp. 41 - 51, 2007. Plasma-Surface Interactions-17.

[75] E. Zawaideh, F. Najmabadi, and R. W. Conn, "Generalized fluid equations for parallel transport in collisional to weakly collisional plasmas," Physics of Fluids, vol. 29 , no. 2, pp. 463-474, 1986. 
[76] R. J. Goldston and P. H. Rutherford, Introduction to Plasma Physics. Taylor \& Francis, 2000.

[77] P. Stangeby and A. Chankin, "Simple models for the radial and poloidal $\vec{E} \times \vec{B}$ drifts in the scrape-off layer of a divertor tokamak: Effects on in/out asymmetries," Nuclear Fusion, vol. 36, no. 7, p. 839, 1996.

[78] R. Pitts, P. Andrew, X. Bonnin, A. Chankin, Y. Corre, G. Corrigan, D. Coster, I. Duran, T. Eich, S. Erents, W. Fundamenski, A. Huber, S. Jachmich, G. Kirnev, M. Lehnen, P. Lomas, A. Loarte, G. Matthews, J. Rapp, C. Silva, M. Stamp, J. Strachan, and E. Tsitrone, "Edge and divertor physics with reversed toroidal field in JET," Journal of Nuclear Materials, vol. 337-339, pp. 146 - 153, 2005. PSI-16.

[79] A. V. Chankin, "Classical drifts in the tokamak SOL and divertor: models and experiment," Journal of Nuclear Materials, vol. 241-243, pp. 199 - 213, 1997.

[80] R. Pitts, J. Horacek, W. Fundamenski, O. Garcia, A. Nielsen, M. Wischmeier, V. Naulin, and J. J. Rasmussen, "Parallel SOL flow on TCV," Journal of Nuclear Materials, vol. 363-365, pp. 505 - 510, 2007. Plasma-Surface Interactions-17.

[81] A. Chankin, D. Coster, N. Asakura, G. Corrigan, S. Erents, W. Fundamenski, H. Mller, R. Pitts, P. Stangeby, and M. Wischmeier, "A possible role of radial electric field in driving parallel ion flow in scrape-off layer of divertor tokamaks," Nuclear Fusion, vol. 47, no. 8, p. 762, 2007.

[82] A. V. Chankin and P. C. Stangeby, "Global circulation of drift flows in the SOL and divertor and its impact on divertor asymmetries," Proceedings of 30th EPS Conference on Contolled Fusion and Plasma Physics, vol. 27A, pp. O-4.1C, 2003.

[83] P. Tamain. Private communication.

[84] P. C. Stangeby and A. V. Chankin, "The ion velocity (Bohm-Chodura) boundary condition at the entrance to the magnetic presheath in the presence of diamagnetic and E x B drifts in the scrape-off layer," Physics of Plasmas, vol. 2, no. 3, pp. 707-715, 1995.

[85] B. D. Scott, "The diamagnetic cancellation in fluid drift dynamics," tech. rep., Max-Planck-Institut für Plasmaphysik, D-85748 Garching, Germany.

[86] A. V. Chankin and P. C. Stangeby, "The effect of diamagnetic drift on the boundary conditions in tokamak scrape-off layers and the distribution of plasma fluxes near the target," Plasma Physics and Controlled Fusion, vol. 36, no. 9, p. $1485,1994$. 
[87] R. A. Pitts, R. Chavan, S. J. Davies, S. K. Erents, G. Kaveney, G. F. Matthews, G. Neill, J. E. Vince, JET-EFDA workprogramme contributors, and I. Duran, "Retarding field energy analyzer for the JET plasma boundary," Review of Scientific Instruments, vol. 74, no. 11, pp. 4644-4657, 2003.

[88] M. Kočan, J. P. Gunn, J.-Y. Pascal, G. Bonhomme, C. Fenzi, E. Gauthier, and J.-L. Segui, "Edge ion-to-electron temperature ratio in the Tore Supra tokamak," Plasma Physics and Controlled Fusion, vol. 50, no. 12, p. 125009, 2008.

[89] G. M. McCracken and P. C. Stangeby, "The interpretation of plasma edge conditions in tokamaks," Plasma Physics and Controlled Fusion, vol. 27, no. 12A, p. $1411,1985$.

[90] A. Tabasso, J. Dowling, J.-W. Ahn, G. Cunningham, A. Kirk, G. McArdle, M. Price, and T. M. Team, "Analysis of the progress to detachment in the divertor of the MAST tokamak," Journal of Nuclear Materials, vol. 313-316, pp. 936 940, 2003. Plasma-Surface Interactions in Controlled Fusion Devices 15.

[91] G. McCracken, M. Stamp, R. Monk, A. Meigs, J. Lingertat, R. Prentice, A. Starling, R. Smith, and A. Tabasso, "Evidence for volume recombination in JET detached divertor plasmas," Nuclear Fusion, vol. 38, no. 4, pp. 619-629, 1998.

[92] U. Wenzel, K. Behringer, A. Carlson, J. Gafert, B. Napiontek, and A. Thoma, "Volume recombination in divertor i of ASDEX Upgrade," Nuclear Fusion, vol. 39, no. 7 , p. 873, 1999.

[93] B. Lipschultz, J. L. Terry, C. Boswell, S. I. Krasheninnikov, B. LaBombard, and D. A. Pappas, "Recombination and ion loss in C-Mod detached divertor discharges," Journal of Nuclear Materials, vol. 266-269, pp. 370 - 375, 1999.

[94] V. Soukhanovskii, R. Maingi, A. Roquemore, J. Boedo, C. Bush, R. Kaita, H. Kugel, B. LeBlanc, S. Paul, G. Porter, and N. Wolf, "Divertor regimes in NSTX," Journal of Nuclear Materials, vol. 337-339, pp. 475 - 479, 2005. PSI-16.

[95] V. Soukhanovskii, R. Maingi, C. Bush, R. Raman, R. Bell, R. Kaita, H. Kugel, C. Lasnier, B. LeBlanc, J. Menard, S. Paul, and A. Roquemore, "Divertor heat flux reduction and detachment experiments in NSTX," Journal of Nuclear Materials, vol. 363-365, pp. 432 - 436, 2007. Plasma-Surface Interactions-17.

[96] V. Soukhanovskii, R. Maingi, D. Gates, J. Menard, S. Paul, R. Raman, A. Roquemore, R. Bell, C. Bush, R. Kaita, H. Kugel, B. LeBlanc, D. Mueller, and the NSTX Team, "Divertor heat flux mitigation in high-performance h-mode 
discharges in the National Spherical Torus Experiment," Nuclear Fusion, vol. 49, no. 9 , p. 095025, 2009.

[97] S. A. Self and H. N. Ewald Physics of Fluids, vol. 9, p. 2486, 1966.

[98] H. P. Summers and M. O'Mullane. The ADAS code, www.adas.ac.uk.

[99] M. Harrison, E. Hoston, and G. Maddison, "AEA fusion report, aea fus 89," tech. rep., Culham Laboratory, 1991.

[100] J. Le and Z. Sizheng, "Investigation of divertor detachment in EAST by 'twopoint' model," Plasma Science and Technology, vol. 9, no. 4, p. 403, 2007.

[101] G. M. McCracken and J. M. Pedgley, "Estimates of the maximum radiate power in a tokamak divertor," Plasma Physics and Controlled Fusion, vol. 35, no. 2, p. 253, 1993.

[102] J. Wesson, Tokamaks, ch. 7, p. 377. Clarendon press, 3 ed., 2004.

[103] A. Thornton. Private communication.

[104] M. Greenwald, "Density limits in toroidal plasmas," Plasma Physics and Controlled Fusion, vol. 44, no. 8, p. R27, 2002.

[105] J. Wesson, Tokamaks, ch. 7, p. 365. Clarendon press, 3 ed., 2004.

[106] J. Wesson, Tokamaks, ch. 6, p. 304. Clarendon press, 3 ed., 2004.

[107] B. LaBombard, J. Goetz, C. Kurz, D. Jablonski, B. Lipschultz, G. McCracken, A. Niemczewski, R. L. Boivin, F. Bombarda, C. Christensen, S. Fairfax, C. Fiore, D. Garnier, M. Graf, S. Golovato, R. Granetz, M. Greenwald, S. Horne, A. Hubbard, I. Hutchinson, J. Irby, J. Kesner, T. Luke, E. Marmar, M. May, P. O'Shea, M. Porkolab, J. Reardon, J. Rice, J. Schachter, J. Snipes, P. Stek, Y. Takase, J. Terry, G. Tinios, R. Watterson, B. Welch, and S. Wolfe, "Scaling and transport analysis of divertor conditions on the Alcator C-Mod tokamak," Physics of Plasmas, vol. 2, no. 6, pp. 2242-2248, 1995.

[108] J. A. Boedo, G. D. Porter, M. J. Schaffer, R. Lehmer, R. A. Moyer, J. G. Watkins, T. E. Evans, C. J. Lasnier, A. W. Leonard, and S. L. Allen, "Flow reversal, convection, and modeling in the DIII-D divertor," Physics of Plasmas, vol. 5, no. 12 , pp. $4305-4310,1998$.

[109] R. D. Monk, A. Loarte, A. Chankin, S. Clement, S. J. Davies, J. K. Ehrenberg, H. Y. Guo, J. Lingertat, G. F. Matthews, M. F. Stamp, and P. C. Stangeby, "Interpretation of ion flux and electron temperature profiles at the JET divertor 
target during high recycling and detached discharges," Journal of Nuclear Materials, vol. 241-243, pp. 396 - 401, 1997.

[110] A. Kirk. Private communication.

[111] A. W. Leonard, G. D. Porter, R. D. Wood, S. L. Allen, J. Boedo, N. H. Brooks, T. E. Evans, M. E. Fenstermacher, D. N. Hill, R. C. Isler, C. J. Lasnier, R. D. Lehmer, M. A. Mahdavi, R. Maingi, R. A. Moyer, T. W. Petrie, M. J. Schaffer, M. R. Wade, J. G. Watkins, W. P. West, and D. G. Whyte, "Radiative divertor plasmas with convection in DIII-D," Physics of Plasmas, vol. 5, no. 5, pp. 1736$1743,1998$.

[112] C. Boswell, Visible Spectroscopic Imaging on the Alcator C-Mod Tokamak. PhD thesis, Massachusetts Institute of Technology, 2003.

[113] R. Scannell. Private communication.

[114] S. W. Lisgo, Interpretive Modelling of the Alcator C-Mod Divertor, ch. 5, p. 102. 2003.

[115] S. W. Lisgo, Interpretive Modeling of the Alcator C-Mod Divertor, ch. 4, p. 74. 2003.

[116] O. Gruber, A. Kallenbach, M. Kaufmann, K. Lackner, V. Mertens, J. Neuhauser, F. Ryter, H. Zohm, M. Bessenrodt-Weberpals, K. Büchl, S. Fiedler, A. Field, C. Fuchs, C. Garcia-Rosales, G. Haas, A. Herrmann, W. Herrmann, S. Hirsch, W. Köppendörfer, P. Lang, G. Lieder, K.-F. Mast, C. S. Pitcher, M. Schittenhelm, J. Stober, W. Suttrop, M. Troppmann, M. Weinlich, M. Albrecht, M. Alexander, K. Asmussen, M. Ballico, K. Behler, K. Behringer, H. S. Bosch, M. Brambilla, A. Carlson, D. Coster, L. Cupido, H. J. DeBlank, S. De Pena Hempel, S. Deschka, C. Dorn, R. Drube, R. Dux, A. Eberhagen, W. Engelhardt, H.-U. Fahrbach, H.U. Feist, D. Fieg, G. Fußmann, O. Gehre, J. Gernhardt, P. Ignacz, B. Jüttner, W. Junker, T. Kass, K. Kiemer, H. Kollotzek, M. Kornherr, K. Krieger, B. Kurzan, R. Lang, M. Laux, M. E. Manso, M. Maraschek, H.-M. Mayer, P. McCarthy, D. Meisel, R. Merkel, H. Murmann, B. Napiontek, D. Naujoks, G. Neu, R. Neu, J.-M. Noterdaeme, G. Pautasso, W. Poschenrieder, G. Raupp, $\mathrm{H}$. Ri, "Observation of continuous divertor detachment in H-mode discharges in ASDEX Upgrade," Phys. Rev. Lett., vol. 74, pp. 4217-4220, May 1995.

[117] J. C. Butcher, Numerical Methods for Ordinary Differential Equations, ch. 3, p. 137. Wiley, 2 ed., 2008. 
[118] H. K. Versteeg and W. Malalasekera, Introduction to Computational Fluid Dynamics: The Finite Volume Method. Longman, 1995.

[119] D. B. Spalding, "A novel finite difference formulation for differential expressions involving both first and second derivatives," International Journal for Numerical Methods in Engineering, vol. 4, no. 4, pp. 551-559, 1972.

[120] B. P. Leonard and J. E. Drummond, "Why you should not use hybrid, power-law or related exponential schemes for convective modelling - there are much better alternatives," International Journal for Numerical Methods in Fluids, vol. 20, pp. 421-442, 1995.

[121] J. D. Hoffman, Numerical Methods for Engineers and Scientists, ch. 3, p. 146. McGraw-Hill, 2001.

[122] C. Hirsch, Numerical Computation of Internal and External Flows, vol. 1. Butterworth-Heinemann, 2 ed., 2007.

[123] van Leer B., "Towards the ultimate conservative difference scheme v. a secondorder sequel to godunov's method," Journal of Computational Physics, vol. 135, pp. 229-248(20), August 1997.

[124] H. K. Versteeg and W. Malalasekera, An Introduction of Computational Fluid Dynamics: The Finite Volume Method, ch. 5, p. 169. Pearson, 2 ed., 2007.

[125] A. Kurganov and E. Tadmor, "New high-resolution central schemes for nonlinear conservation laws and convection-diffusion equations," Journal of Computational Physics, vol. 160, no. 1, pp. 241 - 282, 2000.

[126] P. Wesseling, Principles of Computational Fluid Dynamics. Springer-Verlag, 2001. 